Friedrich Wehrle

Strategische

Marketingplanung in

Warenhäusern 


\section{Friedrich Wehrle}

\section{Strategische Marketingplanung in Warenhäusern}

In der Marketingpraxis und der Marketingwissenschaft sind in den vergangenen Jahren Fragestellungen der strategischen Marketingplanung in den Mittelpunkt des Interesses gerückt. Kenntnisse über Grundbegriffe der strategischen Marketingplanung, Informationssysteme, Aufbau und Systematik von Marketingkonzeptionen, Zielsysteme, Strategieplanungsmethoden, Marketinginstrumentarium sowie Aspekte der organisatorischen Umsetzung gelten heute als selbstverständliches "Rüstzeug" für jeden Praktiker in Führungspositionen - auch im Handelsbereich. Die primare Ausrichtung des Handelsmarketing auf kurzfristige Aktivitäten läßt hier eine Lücke zwischen Anspruch und Wirklichkeit entstehen, die der Autor zu schließen versucht. Die Übertragung erfolgreicher Planungskonzepte, -methoden und -hilfsmittel auf die strategischen Marketingprobleme des Handels (am Beispiel der Betriebsform Warenhaus) soll das Problembewußtsein für die strategische Komponente des Handelsmarketing schärfen, Problemlösungsmöglichkeiten aufzeigen und Ansätze für die Umsetzung in die praktische Strategiearbeit im Handel geben.

Friedrich Wehrle, geboren 1952, studierte von 1972 bis 1976 Betriebswirtschaftslehre an den Universitäten Freiburg und Munster. 1976 Examen als Dipl.-Kfm. in Münster. Tätigkeit als Marketing-Referent in der Investitionsgüterindustrie. Von 1976 bis 1981 Assistent am Institut für Marketing der Westfälischen Wilhelms-Universität Münster. Seit 1981 im Bereich Marketing und Verkauf bei Mohndruck Graphische Betriebe $\mathrm{GmbH}$ in Gütersloh tätig. 
Strategische Marketingplanung in Warenhäusern 


\section{Schriften zum Marketing}

Herausgegeben von Prof. Dr. Heribert Meffert

\section{Band1}

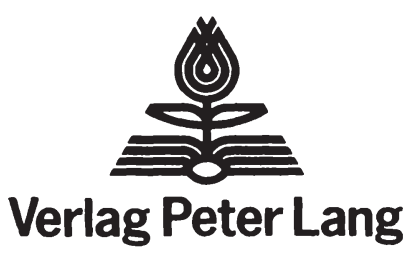

Frankfurt am Main - Bern - New York - Nancy 


\title{
Friedrich Wehrle
}

\author{
Strategische \\ Marketingplanung \\ inWarenhäusern \\ Anwendung der Portfolio-Methode
}

\section{Auflage}

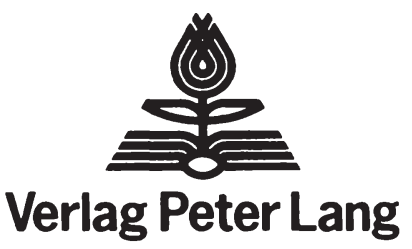

Frankfurt am Main - Bern - New York - Nancy 
CIP-Kurztitelaufnahme der Deutschen Bibliothek

\section{Wehrle, Friedrich:}

Strategische Marketingplanung in Warenhäusern :

Anwendung d. Portfolio-Methode / Friedrich

Wehrle. - 2. Aufl. - Frankfurt am Main ; Bern ;

New York ; Nancy : Lang, 1984.

(Schriften zum Marketing ; Bd. 1)

ISBN 3-8204-6954-0

NE: GT

Open Access: The online version of this publication is published on www.peterlang.com and www.econstor.eu under the international Creative Commons License CC-BY 4.0. Learn more on how you can use and share this work: http://creativecommons.org/licenses/by/4.0.

\section{(c) (i)}

BY

This book is available Open Access thanks to the kind support of ZBW - Leibniz-Informationszentrum Wirtschaft.

ISSN 0721-5819

ISBN 3-8204-6954-0

ISBN 978-3-631-75094-0 (eBook)

(C) Verlag Peter Lang GmbH, Frankfurt am Main 1984

Alle Rechte vorbehal ten.

Nachdruck oder Vervielfältigung, auch auszugsweise, in allen Formen wie Mikrofilm, Xerographie, Mikrofiche, Mikrocard, Offset verboten.

Druck und Bindung: Weihert-Druck GmbH, Darmstadt 
Analysiert man die Veröffentlichung zur strategischen Marketingplanung, so kann man feststellen,

- daß das forscherische Interesse an diesem Themenbereich nach wie vor groß ist

- die wesentlichen Gedanken zunehmend in die Praxis diffundieren

- die Schwerpunkte tendenziell sich in Richtung "Strategisches Management" verlagern

- die Portfolio-Methode in ihrer Bedeutung heute etwas zurückhaltender beurteilt wird.

Diese Aussagen treffen im groben und ganzen allerdings nur auf die industriebezogenen Problemstrukuren zu. Trotz der allgemein weiterhin problematischen Entwicklung im Handel, die eine Stärkung der strategischen Komponente geradezu verlangt, ist in den vergangenen 2 Jahren keine konkret fassbare Weiterentwicklung zu konstatieren.

Insofern liefert diese Arbeit nach wie vor einen Beitrag zum Füllen einer of fensichtlichen Lücke: eingebettet in eine langfristig ausgerichtete marktorientierte Unternehmenspolitik stellt sie die Portfolio-Methode als einen zentralen Baustein der strategischen Marketingplanung dar.

Dr. Wehrle 
Friedrich Wehrle - 978-3-631-75094-0

Downloaded from PubFactory at 01/11/2019 08:26:46AM

via free access 
Vorwort

Die strategische Marketingplanung ist in den vergangenen Jahren stark in den Vordergrund des forscherischen Interesses gerückt. Zahlreiche Veröffentlichungen mit teilweise innovativen und kreativen Denkkonzepten belegen dies. Allerdings liegen diesen Bemühungen in der Regel die Problemstrukturen von Industrieunternehmen zugrunde; der Handel stellt nur im Ausnahmefall das Forschungsobjekt dar. Dies muß zum einen angesichts der bedeutenden Rolle des Handels innerhalb des Marketingsýstems verwundern. Zum anderen kämpft der Handel seit geraumer Zeit mit Problemen (z.B. strukturelle Veränderungen, starke Konzentrationsprozesse, grundlegende Verhaltensänderungen der Käufer), die geradezu nach einer strategischen Neuorientierung der Marketingpolitik verlangen. Dies trifft insbesondere auf die stark betroffene traditionelle Betriebsform Warenhaus $z u$.

Vor diesem Hintergrund ist es die primäre Zielsetzung der Arbeit, einen Beitrag zur strategischen Marketingplanung von Warenhäusern zu leisten. Ausgangspunkt bildet der systematische strategische Marketing - Planungsprozeß sowie das im Industriesektor heute erfolgreich eingesetzte Denkmodell der Portfolio - Methode. Unter beispielhaft empirischer Orientierung soll aufgezeigt werden, ob und in welcher Form die Portfolio - Methode innerhalb des strategischen Planungssystems eines Warenhauses zur Entwicklung von Marketingstrategien beitragen kann. Hierzu müssen die grundlegenden Fragen der Planungssystematik, der Marketingsituation, des Marketingzielsystems, der Definition strategischer Geschäftseinheiten, der Entwicklung von Marketingstrategien unterschiedlichen Globalitätsgrades sowie ihrer organisatorischen Umsetzung diskutiert werden.

Die Erstellung dieser Arbeit verdanke ich in erheblichem Maße der Unterstützung verschiedener Personen. Besonderer Dank gilt an dieser Stelle meinem akademischen Lehrer, Herrn Professor Dr. Heribert Meffert, der die Anregung zu dieser Arbeit gab und die Fertigstellung in dieser Form jederzeit 
förderte. Darüber hinaus danke ich allen meinen Kollegen am Institut für Marketing für ihre vielfältige Unterstützung und Entlastung. Dies gebührt besonders Frau Dr. Simone Pfeiffer - Bohnenkamp, Herrn Dr. Werner Kook und Herrn Dipl.-Kfm. Ralf Becker, die jederzeit zur kritischen Diskussion bereit waren. Nicht zuletzt bin ich meiner Frau dankbar, die in allen Phasen der Erstellung dieser Schrift viel Verständnis gezeigt und mich tatkräftig unterstützt hat. 
Die strategische Unternehmungsplanung hat sich in den vergangenen Jahren $z u$ einem wissenschaftlich bedeutsamen Themenbereich entwickelt. Die vielfältigen forscherischen Bemühungen haben dabei $z u$ einer Reihe auch für die Praxis interessanter Denkmodelle und Konzeptionen geführt. Im Mittelpunkt der Forschungsbemühungen stehen dabei die Problemstrukturen von Herstellerunternehmungen. Dies betrifft insbesondere die Entwicklung heuristischer strategischer Planungsmethoden. Dabei nehmen die sogenannten Portfolio-Methoden als globale Konzepte zur Analyse und Bewertung der industriellen Programmplanung eine besondere Vorrangstellung ein.

Angesichts der grundlegenden strukturellen Veränderungen im Einzelhandel verwundert die Tatsache, daß Probleme der strategischen Unternehmensplanung in diesem Bereich bislang stiefmütterlich behandelt wurden. Dies betrifft insbesondere die Warenhäuser, die als Großbetriebsformen des Handels im Rahmen der marktorientierten Sortimentsplanung erhebliche Probleme der Komplexitätsreduktion zu lösen haben. Dies gilt um so mehr als die veränderten Marktbedingungen gerade in diesem Bereich $z u$ einer Neuorientierung der langfristigen Absatzplanung zwingen.

Diesen Sachverhalt hat der Verfasser der vorliegenden Arbeit klar erkannt. Es ist das Anliegen seiner Arbeit, einen Beitrag zur Entwicklung von Marketingstrategien in Warenhäusern zu leisten, womit er erhebliches Neuland betritt. Insbesondere ist es seine Absicht, $\mathrm{zu}$ überprüfen, inwieweit sich die Portfolio-Methoden auf den Problembereich der strategischen Marketingplanung von Warenhäusern übertragen lassen. Damit besteht der Grundgedanke des Ansatzes darin, die wesentlichen Aktivitäten und Phasen der strategischen Marketingplanung von Herstellerunternehmungen für die Belange der Warenhäuser zu modifizieren. 
Der Einsatz der Portfolio-Methoden erscheint nur dann sinnvoll, wenn einige "Rahmenentscheidungen" vorweg getroffen werden. Folglich müssen überlegungen bezüglich der Bildung strategischer Geschäftseinheiten, der marktorientierten Zielsystems sowie der Positionierung gegenüber Käufern und Konkurrenten vorab getroffen werden.

Diese Rahmenentscheidungen bilden die Grundlage für den modifizierten Einsatz der Portfolio-Methode in Warenhäusern, wobei eine analytisch-sukzessive Vorgehensweise gewählt wird. Dies bedeutet, daß zunächst die Arbeitsweise jeder Portfolio-Variante isoliert dargestellt und kritisch gewürdigt wird, um daran anschließend die isoliert entwickelten Marketing-Basisstrategien insgesamt miteinander $\mathrm{zu}$ vergleichen. Diese bislang in der Literatur noch nicht diskutierte sukzessive Vorgehensweise läßt zum einen die Unterschiede zwischen den auf der Grundlage verschiedener Portfolios entwickelten isolierten Marketing-Basisstrategien besonders deutlich werden. zum anderen gelingt es damit auch Ansatzpunkte zur Verbesserung der Portfolio-Methoden vorzuschlagen, nämlich durch die sukzessive Berücksichtigung mehrerer ziel-, kundensegment- und konkurrenzbezogener Portfolio-Varianten einen Beitrag zur Entwicklung eines konsistenten Systems von Marketing-Basisstrategien in Warenhäusern zu leisten. Gleichzeitig arbeitet der Verfasser auch deutlich die Schwächen dieser Methoden heraus und steckt die Grenzen für ihren Einsatz in Warenhäusern $a b$. Insbesondere macht er deutlich, daß die Portfolio-Methode nur $e \mathrm{i} \quad \mathrm{B}$ a u $\mathrm{s}$ e $\mathrm{i} n$ im Rahmen der gesamten strategischen Marketingplanung darstellt.

Aufgrund des relativ hohen Globalitätsgrades der entwickelten Marketing-Basisstrategien wird die PortfolioMethode um die Konkretisierung durch Marketing-Instrumentalstrategien erweitert. Für einige ausgewählte Basisstrategien 
werden die Aktivitätsniveaus in der Programm-, Distributions-, Kontrahierungs- und Kommunikationspolitik in einem breiten sprektrum beispielhaft aufzeigt. Darüber hinaus werden abschließend einige warenhausspezifische Probleme der organisatorischen Durchsetzung der strategischen Marketingplanung aufgezeigt.

Insgesamt zeigt diese Arbeit die generelle Möglichkeit des Einsatzes strategischer Planungsmethoden, speziell der Portfolio-Methode auf. Sicherlich liegt hier noch ein Potential brach, das dem Handel bei der Bewältigung seiner Probleme gute Hilfe leisten kann. Allerdings mu $B$ von einer unkritischen Ubernahme herstellerspezifischer Denkmodelle gewarnt werden. Vielmehr - und dies zeigt die vorliegende Arbeit in vortrefflicher weise - muB es das Bestreben sein, die besonderen Einsatzbedingungen solcher Methoden im Handel herauszuarbeiten und eine problemadäquate Modifikation vorzunehmen. 
Friedrich Wehrle - 978-3-631-75094-0

Downloaded from PubFactory at 01/11/2019 08:26:46AM

via free access 


\section{Inhaltsverzeichnis}

1. Bedeutung der strategischen Marketingplanung in Warenhäusern

1.1 Strategische Unternehmens- und Marketingplanung in Hersteller- und Handelsunternehmungen

1.11 Gegenstand und begriffliche Abgrenzung

1.12 Notwendigkeit der strategischen Unternehmens- und Marketingplanung im Handel

1.2 Strategische Marketingplanung in Warenhäusern

1.21 Warenhäuser im Spannungsfeld der Betriebsformen

1.22 Gegenstand und Besonderheiten der strategischen Marketingplanung in Warenhäusern

1.3 Bedeutung der Portfolio-Methode für die strategische Marketingplanung in Warenhäusern

1.4 Zielsetzung und Gang der Untersuchung

2. Abgrenzung und Bildung strategischer Geschäftseinheiten in Warenhäusern als Gegenstand der Portfolio-Planung

2. 1 Bedeutung der strategischen Geschäftseinheiten in Warenhäusern

2.11 Darstellung und Würdigung des Konzepts der strategischen Geschäftseinheiten

2.12 Übertragbarkeit des Konzepts für Warenhäuser

2.2 Bildung strategischer Geschäftseinheiten in Warenhäusern

2. 21 Strategische Geschäftseinheiten in Warenhäusern bei isolierter Filialsicht

2.22 Strategische Geschäftseinheiten in Warenhäusern bei isolierter Warensicht

2.23 Vergleichende Beurteilung der isolierten Ansätze 
Seite

2.3 Duale Interpretation strategischer Geschäftseinheiten in Warenhäusern und ihre Implikationen für die strategische Marketingplanung

3. Ziele und Positionierung als Rahmenentscheidungen für die Portfolio-Planung in Warenhäusern

3.1 Festlegung von Marketingzielen in Warenhäusern

3.11 Funktionen und Anforderungen marktorientierter Ziele in Warenhäusern

3.12 Relevante Ziele für die strategische Marketingplanung in. Warenhäusern

3.13 Bildung marketingorientierter $\mathrm{Ziel-}$ systeme in Warenhäusern

3.2 Positionierung von Warenhäusern im Hinblick auf Käufersegmente

3.21 Demographische und psychographische Struktur der Zielgruppen von Warenhäusern 101

3.22 Zielgruppenstrategien von Warenhäusern 108

3. 3 Positionierung von Warenhäusern im Konkurrenzumfeld

3.31 Typen von Konkurrenzstrategien in Warenhäusern

3.32 Analyse der Marktposition von Warenhäusern im Konkurrenzumfeld

4. Entwicklung von Marketing-Basisstrategien auf der Grundlage der Portfolio-Methode

4.1 Ermittlung von Portfolio-Varianten für Warenhäuser auf der Basis der Rahmenentscheidungen

4.2 Anwendung analytischer Portfolio-Varianten in Warenhäusern

4.21 Warengruppen- und Filialgruppenstrategien auf der Basis des Image-Ertragskraft-Portfolios 
Seite

4.22 Warengruppen- und Filialgruppenstrategien auf der Basis des MarktanteilsErtragskraft-Portfolios

4.23 Warengruppen- und Filialgruppenstrategien auf der Basis des MarktanteilsMarktwachstums-Portfolios

4.24 Warengruppen- und Filialgruppenstrategien auf der Basis des MarktwachstumsUnternehmenswachstums-Portfolios

4.3 Anwendung synthetischer Portfolio-Varianten in Warenhäusern

4.31 Warengruppen- und Filialgruppenstrategien auf der Basis des LebenszyklusWettbewerbspositions-Portfolios

4.32 Warengruppen- und Filialgruppenstrategien auf der Basis des Marktattraktivitäts-Wettbewerbsvorteile-Portfolios

4. 4 Zusammenfassung und kritische Würdigung der Eignung der Portfolio-Methode zur Entwicklung von Basisstrategien in Warenhäusern

4.41 Vergleich der isoliert entwickelten Marketing-Basisstrategien für die Warengruppen des Warenhauses $\mathrm{X}$

4.42 Kritische Würdigung und Ansatzpunkte zur Weiterentwicklung der PortfolioMethode in Warenhäusern

5. Konkretisierung der Basisstrategien durch Marketing-Instrumentalstrategien

5.1 Strukturierung der Marketing-Instrumentalstrategien

5.11 Systematisierung der Marketinginstrumente in Warenhäusern

5.12 Zusammenhang zwischen Marketing-Basisstrategien und Instrumentalstrategien

5.2 Entwicklung sortimentsoolitischer Marketingstrategien von Warenhäusern

5.21 Sortimentsstrategien in Warenhäusern

5.22 Marken-, Qualitäts- und Servicestrategien in Warenhäusern 
Seite

5.3 Entwicklung distributionspolitischer Marketingstrategien von Warenhäusern

5.31 Standortbezogene Strategien 203

5.32 Verkaufsstättenbezogene Strategien 210

5. 4 Entwicklung kontrahierungspolitischer Strategien von Warenhäusern

5.5 Entwicklung kommunikationspolitischer Strategien von Warenhäusern

5. 6 Problem der Auswahl und Kombination der Marketing-Instrumentalstrategien in Warenhäusern

6. Implikationen für die Durchsetzung der strategischen Marketingplanung in Warenhäusern

6.1 Ansätze zur Koordination und Integration der Marketingstrategien

6.11 Darstellung und kritische würdigung idealtypischer Planungsverfahren in Warenhäusern

6.12 Planung von Marketingstrategien in Warenhäusern nach dem Gegenstromverfahren

6.13 Horizontale Abstimmung (Koordination) von Marketingstrategien auf der SGE-Ebene 249

6.2 Stufenweise Realisation eines hierarchischdualistischen Marketing-Planungssystems in Warenhäusern 
Abbildungsverzeichnis

Seite

Abbildung 1: ProzeB der strategischen Marketingplanung in Warenhäusern

Abbildung 2: Grundstruktur des Portfolio-Konzeptes

Abbildung 3: Beispiel zur Gruppierung von Filialen

Abbildung 4: Sortimentshierarchie und strategische Marketingplanung

Abbildung 5: Beurteilung isoliert gebildeter strategischer Geschäftseinheiten

Abbildung 6: Marketingstrategietypen auf der Basis einer Filialsystem-SortimentsMatrix

Abbildung 7: Beispielhaftes marketingorientiertes Zielsystem von Warenhäusern

Abbildung 8: Zusammenhang zwischen Umsatz und Erfolgsziel bei dualistischer Aufbauorganisation

Abbildung 9: Ausschnitt eines marketingorientierten Zielsystems von Warenhäusern

Abbildung 10: Grundstruktur des Marktsegmentierungs-Konzepts 
Seite

Abbildung 11: Dreidimensionales Positionierungsmodell von (Nicht-) Warenhauskunden auf der Basis psychographischer Variablen

Abbildung 12: Typologisierung von Kunden ausgewählter Betriebsformen

Abbildung 13: Entscheidungsspektrum Zielgruppenstrategie

Abbildung 14 : Systematisierung von Konkurrenzstrategien eines Warenhauses

Abbildung 15 : Leistungsprofil ausgewählter Betriebsformen

Abbildung 16 : Beurteilung von vier Warenhauskonzernen anhand ausgewählter Merkmale

Abbildung 17: Image-Ertragskraft-Portfolio der Warengruppen des Warenhauses $\mathrm{X}$

Abbildung 18: Marktanteils-Ertragskraft-Portfolio der Warengruppen des Warenhauses $\mathrm{X}$

Abbildung 19 : Marktanteils-Marktwachstums-Portfolio der Warengruppen des Warenhauses $\mathrm{X}$

Abbildung 20: Wachstums-Portfolio der Warengruppen des Warenhauses $\mathrm{X}$

Abbildung 21: Lebenszyklus-WettbewerbspositionsPortfolio der Warengruppen des Warenhauses $\mathrm{X}$

Abbildung 22: Marktattraktivitäts-Wettbewerbsvorteile-Portfolio der Warengruppen des Warenhauses $\mathrm{X}$ 
Seite

Abbildung 23: Zielorientierte Modifizierung von Basisstrategien

Abbildung 24: Systematik der Marketing-Instrumente in Warenhäusern

Abbildung 25: Mehrstufig-heuristische Vorgehensweise zur Ableitung von Marketing-Instrumentalstrategien

Abbildung 26: Zusammenhang zwischen Sortimentsniveau und Preislagenstrategie

Abbildung 27: Aktivitätenniveau (Netzstrategie) der Marketinginstrumente in Warenhäusern

Abbildung 28: Heuristische Vorgehensweise zur Kombination der Marketing-Instrumentalstrategien in Warenhäusern

Abbildung 29: Strategieplanung in Warenhäusern nach dem Gegenstromverfahren 


\section{Tabellenverzeichnis}

Seite

Tabelle 1: Abgrenzungskriterien und Anforderungen an strategische Geschäftseinheiten

Tabelle 2: Anzahl der Filialen der Warenhausunternehmen in den Bundesländern stand 31.12 .1978

Tabelle 3: Beispielhafte Abgrenzung von Sortimentsstrukturen

Tabelle 4: Demographische Beschreibung von Warenhauskunden und Nichtwarenhauskunden

Tabelle 5: Relevante Faktoren der Marktattraktivität und relativen Wettbewerbsvorteile für Warenhäuser

Tabelle 6: Schema eines Punktbewertungsverfahrens zur Beurteilung der Marktattraktivität

Tabelle 7: Isolierte Entwicklung der Marketing-Basisstrategien mit Hilfe des Image-Ertragskraft-Portfolios und des Marktanteils-Ertragskraft-Portfolios 174

Tabelle 8: Isolierte Entwicklung der MarketingBasisstrategien mit $\mathrm{Hilfe}$ des Marktanteils-Marktwachstums-Portfolio und des Marktwachstums-Unternehmenswachstums-Portfolios

Tabelle 9: Isolierte Entwicklung der MarketingBasisstrategien mit Hilfe des Lebenszyklus-Wettbewerbs-Portfolio und des Marktattraktivitäts-Wettbewerbsvorteile-Portfolios 
Tabelle 10: Endgültige Marketing-Basisstrategien auf der Basis eines sukzessiven Strategienvergleichs

Tabelle 11: Umsatz pro qm nach Filialgrößenklassen

Tabelle 12: Wachstumsraten relevanter Größen als Prämissen für die Strukturanalyse

Tabelle 13: Hypothetisches Beispiel einer warengruppenbezogenen Strukturprojektion

Tabelle 14: Beispiel für ein Punktbewertungsverfahren zur Bewertung und Auswahl von Strategien 


\section{Abkürzungsverzeichnis}

a.a.o.

$\mathrm{Abb}$.

AG

AMA

ASW

Auf 1 .

BAG

BBE

Bd.

BddW

bzw.

DBW

Diss.

DOB

dt.

DU

EIASM

Erg.

et a 1 .

etc.

e.V.

evtl.

f., ff.

FAZ

$\mathrm{FfH}$

ggf.

GfK

GfM

HaKa

HBR

Hrsg.

hrsg. v. am angegebenen Ort

Abbildung

Aktiengesellschaft

American Marketing Association

Absatzwirtschaft

Auflage

Bundesarbeitsgemeinschaft der Mittelund Großbetriebe des Einzelhandels e.V., Köln

Betriebswirtschaftliche Beratungsstelle für den Einzelhandel

Band

Blick durch die Wirtschaft

beziehungsweise

Die Betriebswirtschaft

Dissertation

Damenoberbekleidung

deutsch

Die Unternehmung

European Institute for Advance

Ergänzung

et altera

et cetera

eingetragener Verein

eventuell

folgende Seite, Seiten

Frankfurter Allgemeine Zeitung

Forschungsstelle für den Handel

gegebenenfalls

Gesellschaft für Konsumforschung

Gesellschaft für Markt- und Meinungsforschung

Herrenkleidung

Harvard Business Review

Herausgeber

herausgegeben von 
i.e.s.

i.d. R.

ISB

Jg •

JoM

JoR

LRP

MM

N.J.

No., Nr.

o.V.

O.J.

PIMS

qm

ROI

S.

SB

SBU

SGE

Sp.

Tab.

u.a.

usw.

U.U.

$\mathrm{v}$.

Vol.

WISU

Wist

W\&V

z.B.

$\mathrm{ZfB}$

$\mathrm{ZfbF}$

$\mathrm{ZO}$ im engeren Sinn

in der Regel

Institut für Selbstbedienung

Jahrgang

Journal of Marketing

Journal of Retailing

Long Range Planning

Manager Magazin

New Jersey

Nummer

ohne Verfasser

ohne Jahr

Profit Impact of Market Strategies

Quadratmeter

Return on Investment

Seite

Selbstbedienung

Strategic Business Unit

Strategische Geschäftseinheit

Spalte

Tabelle

unter anderem

und so weiter

unter Umständen

von

Volume

Das Wirtschaftsstudium

Wirtschaftswissenschaftliches Studium

Werben \& Verkaufen

zum Beispiel

Zeitschrift für Betriebswirtschaft

zeitschrift für betriebswirtschaftliche Forschung

Zeitschrift für Organisation 
Friedrich Wehrle - 978-3-631-75094-0

Downloaded from PubFactory at 01/11/2019 08:26:46AM

via free access 
1 Bedeutung der strategischen Marketingplanung in

Warenhäusern

1.1 Strategische Unternehmens- und Marketingplanung in Hersteller - und Handelsunternehmungen

\subsection{Gegenstand und begriffliche Abgrenzung}

Vor dem Hintergrund einer sich schneller wandelnden und zunehmend komplexeren Umwelt wird das Problembewußtsein für strategische Fragestellungen geschärft und die Bemühungen $\mathrm{zu}$ ihrer Lösung intensiviert. Insbesondere in den vergangenen zehn Jahren wurde der wissenschaftlichen Erforschung und praktischen Anwendung der strategischen Unternehmens- und Marketingplanung verstärkte Aufmerksamkeit zuteil. Das Ergebnis dieser vielfältigen forscherischen Bemühungen sind eine Fülle von Denkmodellen und Konzepten, die sich inhaltlich in der Bearbeitung spezifischer Schwerpunkte unterscheiden und teilweise zeitbedingt die Probleme aus unterschiedlichen Blickwinkeln heraus zu lösen versuchen.

Die strategische Unternehmensplanung befaßt sich mit der globalen Analyse der Erfolgsquellen und der Entwicklung langfristig angelegter Konzepte zur zukunftssicherung ${ }^{1}$. Dabei dient die strategische Planung primär der Beein-

1 Damit wird hier weitgehend der Auffassung von Gälweiler gefolgt. Vgl. hierzu: Gälweiler, A., Unternehmensplanung, Frankfurt / New York 1974, S. $133 \mathrm{ff}$. und S. $229 \mathrm{ff.} \mathrm{(im} \mathrm{folgenden} \mathrm{zitiert} \mathrm{als:} \mathrm{Gälweiler,} \mathrm{A.,}$ Unternehmensplanung). Ein materiell ähnliches Verständnis der strategischen Unternehmensplanung ist heute bei den meisten Autoren, insbesondere auch im englischsprachigen Raum zu finden. Vgl. zu einer detaillierten Darstellung verschiedener Begriffsabgrenzungen: Hadaschik, M., Die Einsatzbedingungen organisierter langfristiger Unternehmensplanung, Diss., Berlin 1979, S. $27 \mathrm{ff.}$. 
flussung des Verhaltens sozio-technischer systeme in einer ständigen Veränderungen unterworfenen Umwelt ${ }^{1}$. Interpretiert man die strategische Unternehmensplanung als umfassenden, iterativen und dynamischen Planungspro$z e \beta$, sind dabei die folgenden interdependenten Teilprobleme zu lösen ${ }^{2}$ :

(1) Definition des Geschäftsfeldes bzw. der Geschäftsfelder (Abgrenzung und Festlegung von Produkt-Marktkombinationen).

(2) Die Bestimmung der "Mission" des Geschäfts bzw. der Geschäftsfelder (Festlegung strategischer Ziele bzw. Schlüsselfaktoren wie z.B. Umsatzwachstum, Marktanteile, ROI, Cash flow).

(3) Die Formulierung funktionaler Strategien (Marketing, Forschung und Entwicklung, Produktion).

(4) Die Budgetierung (Festlegung und Allokation der Ressourcen).

Neben der Sicherung der marktlichen Erfolgsvoraussetzungen soll die strategische Unternehmensplanung die Reaktionsoder Antizipationsfähigkeit (Flexibilität) der Unternehmung erhöhen ${ }^{3}$, das Risiko von langfristig wirksamen Entscheidungen vermindern, die Komplexität der zu berücksichtigenden relevanten internen (z.B. soziale Beziehungen,

1 Vgl. Zahn, E., Strategische Planung zur Steuerung der langfristigen Unternehmenspolitik, Berlin 1979, S. 10.

2 Vgl. Meffert, H., Strategische Planung unter besonderer Berücksichtigung von Marktsättigung und Rezession, in: ASW,6/1980, S. 89-97, hier S. 89 (im folgenden zitiert als: Meffert, H., Strategische Planung).

$3 \mathrm{Vgl}$. ebenda, S. 89; Zahn spricht hier von den grundlegenden Zwecken der "Vorbereitung auf Eventualitäten" sowie dem "Entdecken und Generieren von Potentialitäten"; vgl. Zahn, E., a.a.O., S. 9. 
Interessengruppen, Potentiale) und externen Faktoren (z.B. Ökonomische, politische, soziale, technische Entwicklungen) reduzieren und zur Ausnutzung von Synergien ${ }^{1}$ (im Produktions-, Marketing-, Finanz- oder Managementbereich) beitragen.

Interpretiert man als primäre Aufgabe der strategischen Marketingplanung die Beantwortung der Frage, welche Produkte in welchen Märkten mit welchen Marketingaktivitäten zu welchem Zeitpunkt vertrieben werden sollen 2 , so wird deutlich, daß die strategische Marketingplanung nicht nur eine funktionale Komponente, sondern das Kernstück der strategischen Unternehmensplanung darstellt ${ }^{3}$. Im Rahmen der marktorientierten Handlungsweise von Unternehmungen beeinflussen Fragestellungen der strategischen Marketingplanung alle Teilprobleme, die das Aufgabenfeld der strategischen Unternehmensplanung determinieren ${ }^{4}$. Damit kommt ihr eine zentrale Bedeutung zu.

Diese zentrale Stellung der strategischen Marketingpla-

1 Vgl. hierzu Ansoff, H.I., Management Strategie, München 1966, S. $97 \mathrm{ff}$. (im folgenden zitiert als Ansoff, H.I., Management Strategie).

2 Vgl. Meffert, H., Strategische Planung, a.a.o., S. 89.

3 Diese Ansicht vertritt auch Koch, der dem Absatz eine Schlüsselstellung im Rahmen der strategischen Planung zuerkennt und Strategien primär durch Produktlinien und Märkte kennzeichnet. Vgl. Koch, H., Die zentrale Globalplanung als Kernstück der integrierten Unternehmensplanung, in: Wild, J. (Hrsg.), Unternehmensplanung. Reader + Abstracts, Reinbek 1975, S. 145-153, hier S. 148 (im folgenden zitiert als: Koch, H., Globalplanung).

4 Vgl. hierzu auch Henzler, H., Strategisches Marketing als Impulsgeber für die 8oer Jahre, in: $\mathrm{ZfbF}$ Sonderheft II, 1980, S. 70-86. 
nung wird durch die Analyse der Entwicklung ${ }^{1}$ der strategischen Unternehmens- und Marketingplanung deutlich. In den 6oer Jahren standen strategische probleme im Mittelpunkt, die sich im wesentlichen auf das Erkennen von Disharmonien in den Unternehmens-Markt-Beziehungen konzentrierten und auf der Basis von Unternehmens- und Umweltanalysen Lösungen erarbeiteten, die nahezu ausschließlich im Bereich rationaler Wirtschaftstätigkeit lagen und verhaltenswissenschaftliche Aspekte vernachlässigten. Die unter diesem Blickwinkel des strategischen Problems erarbeiteten Denkmodelle und Konzepte beinhalten eine Reihe von Grundfragen, die die Gesamtkonzeption und den Planungsprozeß, einzelne Strategietypen sowie die Umwelt betreffen ${ }^{2}$. Diesen Ansätzen ist der Grundgedanke gemeinsam, daß das überleben der Unternehmung das zentrale

1 Einen guten Überblick über die Entwicklung der strategischen Planung findet sich z.B. bei Bircher, B., Langfristige Unternehmensplanung, Bern/Stuttgart 1976, S. 15 ff.; Ewing, D.W. (Hrsg.), Long-Range Planning for Management, New York 1964, Mockler, R.J., Theory and Practice of Planning, in: HBR, March-April 1970, pp. 148-159; Shanklin, W.L., Strategic Business Planning: Yesterday, Today and Tomorrow, in: LRP, Oct. 1979, S. 7-14; Zahn, E., a.a.O., S. $19 \mathrm{ff}$.

2 Aus der Vielzahl der Veröffentlichungen sind nach Meinung des Autors folgende Beiträge aufgrund ihrer wissenschaftlichen Leistung im Hinblick auf den Erkenntnisfortschritt hervorzuheben: Ackoff, R.L., Unternehmensplanung, München 1972; Aghte, K., Strategie und Wachstum der Unternehmung, Baden-Baden 1972; Ansoff, H.I., Management Strategie; Anthony, R.N., Planning and Control Systems, Boston 1965; Argenti, J., Die Langfristplanung im Unternehmen, München 1970; Aurich, W. , Schröder, H.K., System der Wachstumsplanung im Unternehmen, München 1972; Borschberg, E., Die Diversifikation als Wachstumsform der industriellen Unternehmung, Bern 1969; Chandler, A.D., Strategy and Structure, Cambridge, Mass. 1962; Payne, B., Planning for Company Growth of the Firm, New York 1963; Penrose, E.T., The Theory of the Growth of the Firm, Oxford 1966; Scott, B.W., Long-Range Planning in American Industry, New York 1965; Steiner, G.A., Top Management Planning, London 1969; Vancil, R.F., et al. (Hrsg.), Formal Planning Systems, Harvard Business School, Boston 1969 . 
Problem darstelle und hierzu die Anpassung der ProduktMarkt-Strategie an die Umweltbedingungen entsprechend der Heuristik "Stärken betonen - Schwächen vermeiden" zu erfolgen habe' ${ }^{1}$. Entsprechend dieser Denkweise konzentrierten sich die Lösungsansätze auf partielle Bereiche, d.h., es wurde primär der Produkt-Markt-Bereich betrachtet und die Umwelt weitgehend auf potentielle Kunden sowie potentielle Konkurrenten reduziert ${ }^{2}$. Insbesondere die seit Ende der 6oer Jahre wichtiger werdenden Veränderungen der Umwelt im ökonomischen (Kaufkraft, Inflation usw.), ökologischen (Ressourcenverknappung, Umweltprobleme usw.), sozialen (Käuferverhalten, Änderung der sozialen Schichtung, Arbeitslosigkeit usw.) und politischen Bereich (Gesetzesflut, Wirtschafts- und Steuerpolitik usw.) wurden ebenso vernachlässigt wie Änderungen innerhalb der Unternehmung (Mitbestimmung, soziale Absicherung, stärkeres Streben nach Selbständigkeit und Selbstverwirklichung usw.).

Diese Entwicklungen forderten eine Neuformulierung des strategischen Problems ${ }^{3}$, das die bis dahin zu konstatierende einseitige Ausrichtung überwindet. Diese Neuformulierung setzt zunächst an der Ausweitung der Dimensionen des strategischen Problems an und fordert insbesondere die Berücksichtigung

- der Bemühungen um die Übereinstimmung zwischen Umwelt, Strategie und interner Konfiguration (z.B. Führungssystem, Potentiale) sowie der zeitlichen Abfolge des Anpassungsprozesses

1 Vgl. Roventa, P., Portfolio-Analyse und Strategisches Management, München 1979, S. 17.

2 Vgl. ebenda, S. 18.

3 Ansoff u.a. prägten hierfür den Begriff des "strategischen Management". Vgl. Ansoff, H.I., Hayes, R.L., Introduction, in: Ansoff, H.I., Declerck, R.I., Hayes, R.L., (Hrsg.), From Strategic Planning to Strategic Management, London usw. 1976, S. 1-12. Inzwischen hat Ansoff den Grundgedanken weiterentwickelt. Vgl. hierzu Ansoff,H.I., Strategie Management,London usw. 1979,S.8 ff. (im folgenden zitiert als: Ansoff,H.I.,Strategic Management). 
- der Implementierung der strategischen Unternehmens- bzw. Marketingplanung sowie

- einer Vielzahl neuer relevanter Variablen aus dem internen und externen Bereich der Unternehmung.

Hinzu kommt der Versuch, den immer rascher akzelerierenden Wandel der Umwelt adäquat zu handhaben. Es wird zunehmend wichtiger, aufkommende Turbulenzen (Diskontinuitäten) ${ }^{1}$ frühzeitig zu erkennen ${ }^{2}$ und ihnen mit adäquaten Aktivitäten zu begegnen.

Diese neuen Anforderungen an die strategische Planung brachten eine Verlagerung der Forschungsschwerpunkte in der strategischen Unternehmens- und Marketingplanung mit sich. Heute stehen primär Fragestellungen des Aufbaus der strategischen Planung und ihrer Integration in das Führungssystem ${ }^{3}$, die Berücksichtigung der norma-

1 Drucker prägte den Begriff des "Zeitalters von Diskontinuitäten. Vgl. Drucker, P.F., The Age of Discontinuity, New York 1969, S. 9 ff... Ansoff greift ihn auf und erhebt die dahinterstehende Problematik zum zentralen Gegenstand der strategischen Planung. Vgl. hierzu die folgenden Beiträge: Ansoff, H.I., Managing, Surprise and Discontinuity - Strategic Response to Weak Signals, in: ZfbF 1976, S. 129-152 (im folgenden zitiert als: Ansoff, H.I., Weak Signals); Derselbe, Planned Management in Turbulent Change, in: EIASM - Working Paper 78-3, Brüssel 1978 (im folgenden zitiert als: Ansoff, H.I., Planned Management).

2 Dieser Aspekt wird speziell unter dem Stichwort "Frühwarnsysteme" analysiert. Vgl. zum aktuellen Stand der Forschung: Albach, H., Hahn, D., Mertens, P., Frühwarnsysteme, in: ZfB, Erg. Heft 2/1979; Rieser, I., Frühwarnsysteme für die Unternehmenspraxis, München 1980, sowie die dort angegebene Literatur.

$3 \mathrm{Vgl}$. exemplarisch Hinterhuber, H., Strategische Unternehmensführung, 2. Auf1., Berlin/New York 1980; Koch, H., Globalplanung, a.a.O., S. $145 \mathrm{ff}$; Derselbe, Aufbau der Unternehmensplanung, Wiesbaden 1977 (im folgenden zitiert als: Koch, H., Aufbau); Lorange, P., Vancil, R.F., How to design a Strategic Planning System, in: HBR Sept.- Oct. 1976, S. 75-81; Töpfer, A., Planungsund Kontrollsysteme industrieller Unternehmungen, Berlin 1976, insbes. S. 87 ff.; Lorange, P., Vancil, R.F., Strategic Planning in Diversified Companies, in: HBR Jan.-Feb. 1975, S. 75-81. 
tiven Komponente (Wertproblematik), die Betrachtung individueller und gruppenspezifischer Prozesse ${ }^{1}$, die Organisation $^{2}$ der strategischen Unternehmensplanung und vor allem ihre methodische und technische Unterstützung ${ }^{3}$ im Vordergrund 4 .

Die Erweiterung des strategischen Planungsproblems findet ihren Niederschlag notwendigerweise ebenso bei der

1 Vgl. hierzu den grundlegenden Aufsatz von Kirsch, W., Bamberger,I., Strategische Unternehmensplanung, Rationalität und Philosophien der politischen Planung, in: $\mathrm{ZfB} 1976, \mathrm{~S} .341-356$ sowie die dort angegebene Literatur.

2 Vgl. den guten Überblick bei Gabele, E., Unternehmensstrategie und Organisationsstruktur, in: ZO 4/1979, S. 183-190 sowie die dort angegebene Literatur. Hiermit hängen auch eng die Probleme der Persönlichkeit und Fähigkeiten von Managern für die strategische Planung zusammen. Vgl. hierzu z.B. Ansoff, H.I., The Changing Manager, in: Ansoff, H.I., Declerck, R.P., Hayes, R.L. (Hrsg.), From Strategic Planning to Strategic Management, London usw. 1976, S. 181-197; Wissema, J.G., Van der Pol, H.W., Messer, H.M., Strategic Management Archetypes, in: Strategic Management Journal, Vol.1 $(1980)$, S. 37-47.

3 Hier sind insbesondere die modell- und computergestützte Unternehmensplanung sowie die vielfältigen Ansätze der Portfolio-Methode zu nennen. Einen guten úberblick zum Stand der Wissenschaft geben für den ersten Bereich: Grochla, E., Szyperski, N. (Hrsg.), Modell- und Computergestützte Unternehmensplanung, Wiesbaden 1973; Hansen, H.R. (Hrsg.), Computergestützte Marketingplanung, München 1974; Plötzeneder, H.D. (Hrsg.), Computergestützte Unternehmensplanung, Stuttgart usw. 1977. Zum zweiten Bereich vgl. exemplarisch Szyperski, N., Winand, U., a.a.O., S. $123 \mathrm{ff}$..

4 Allerdings haben die vielfältigen Forschungsanstrengungen bis heute nur einen begrenzten Erkenntnisfortschritt gebracht, so daß nahezu einhellig die Meinung vertreten wird, heute lediglich von einer rudimentären Planungstheorie sprechen zu können. Vgl. zur kritischen Auseinandersetzung mit der Planungstheorie z.B.: Kirsch, W. , Bamberger, I., a.a.O., S. 341 ff.; Szyperski, N., Planungswissenschaft und Planungspraxis, in: Wild,J.(Hrsg.), Unternehmensplanung, Reinbek, 1975, S.58-75 (im folgenden zitiert als: Szyperski, N., Planungswissenschaft); Szyperski,N.,Welters,K., Grenzen und ZweckmäBigkeit der Planung, in: Die Unternehmung 1976, S. 165-283, hier S. $267 \mathrm{ff.;}$ Welters,K.,Zum Problembegriff einer Theorie strategischer Unternehmensplanung, in: ZO 3/1978,S.135142, hier S. 135; Zahn,E., a.a.O., S. $42 \mathrm{ff} .$. 
Ableitung konkreter Marketingstrategien ${ }^{1}$, die nach wie vor das Kernstück der strategischen Marketingplanurig darstellen. Unter einer Marketingstrategie ist dabei ein bedingter, langfristiger, globaler Verhaltensplan zur Erreichung der Unternehmungs- und Marketingziele $\mathrm{zu}$ verstehen ${ }^{2}$. Die Marketingstrategie ist insofern als bedingt $\mathrm{zu}$ bezeichnen, als sie in einer spezifischen internen und externen Situation unter einem gegebenen Informationsstand festgelegt wird. Ihre Kennzeichnung als langfristig setzt an der zeitlichen Wirksamkeit an: Marketingstrategien müssen mehrere Planungsperioden (i. d.R. Jahre) wirksam sein. Mit der Globalität ist die Tatsache angesprochen, daß der Gegenstand von Marketingstrategien meist auf einem hohen Aggregationsgrad zu sehen ist. Durch den Verhaltensbegriff wird die Handlungsorientierung deutlich; die Marketingstrategie legt die Handlungen der Unternehmung fest. Durch den Zusatz "Plan" soll die Verbindlichkeit der Marketingstrategie verdeutlicht werden. In der Regel liegen als Ergebnis dokumentierte, für nachfolgende Planungsebenen verbindliche Planungsdokumente vor.

Nahezu allen Veröffentlichungen zur Marketingplanung und zur Ableitung von Marketingstrategien in den vergangenen Jahren liegen mehr oder weniger explizit die strategischen Problemstrukturen industrieller Unternehmungen zugrunde. Offensichtlich ist der Zwang zur Beschäftigung

$1 \mathrm{Vgl.} \mathrm{zum} \mathrm{etymologischen} \mathrm{Ursprung} \mathrm{des} \mathrm{Begriffs} \mathrm{"Strate-}$ gie" z.B. Wündrich, H., Strategie - Begriff kritisch betrachtet, in: ASW 11/1977, S. 84-9o, hier S. 84 .

2 Vgl. Meffert, H., Strategische Planung, a.a.O., S. 89. Vgl. auch die materiell ähnliche Interpretation des Strategiebegriffs bei Ahlert, D., Grundzüge des Marketing, Düsseldorf 1980,S. 53 ff. (im folgenden zitiert als: Ahlert, D., Marketing). Zu weiteren Interpretationen des (Marketing-) Strategiebegriffs vgl. z.B. Ansoff,H.I., Management Strategie, a.a.O., S. $125 \mathrm{ff} . ;$ Agthe, K., a.a.0., S. 17; Kotler, P., Marketing Management. Analysis Planning and Control, 4. Aufl., Englewood Cliffs, N.J. 1980, S. 272; Pümpin, C.B., a.a.O., S. 76; Scheuplein, H., Unternehmensstrategie, Wiesbaden 1970, S. $17 \mathrm{f} .$. 
mit strategischen Fragestellungen angesichts der wachsenden Komplexität und der zunehmenden Dynamik sozialer Systeme sowie den unmittelbar daraus resultierenden Führungsproblemen in Herstellerunternehmungen heute klar erkannt worden ${ }^{1}$. Auf der anderen Seite ist diese Problematik in anderen Wirtschaftsbereichen und insbesondere im Handel noch kaum adäquat berücksichtigt, was zum einen $\mathrm{zu}$ dem früher geringen Wettbewerbsdruck und zum anderen in der bisherigen Dominanz der Hersteller im Absatzkanal begründet liegt.

1.12 Notwendigkeit der strategischen Unternehmens- und Marketingplanung im Handel

Die im Vergleich zum Industriebereich geringe wissenschaftliche und praktische Auseinandersetzung mit den Phänomenen der strategischen Planung im Handel ${ }^{2}$ muß

1 Vgl. zu ähnlichen Gründen für die Notwendigkeit zur strategischen Unternehmens- und Marketingplanung in Herstellerunternehmungen: Albach, H., Strategische Unternehmensplanung bei erhöhter Unsicherheit, in: ZfB 48 (1978), S. 702-715, hier S. 7o2 f. (im folgenden zitiert als: Albach, H., Unsicherheit); Gälweiler, A., Unternehmenssicherung und strategische Planung, in: $\mathrm{ZfbF} 28$ (1976), S. 362-379, hier S. 362 (im folgenden zitiert als Gälweiler, A., Unternehmenssicherung); Zahn, E., a.a.0., S. 11. Vgl. auch Koch, der ähnliche Faktoren unter den Begriff der Wirtschaftsunruhe subsummiert. Vgl. Koch, H., Wirtschaftsunruhe und Unternehmensplanung, in: $\mathrm{zfbF}$, 28 (1976), S. 330-341 (im folgenden zitiert als Koch, H. , Wirtschaftsunruhe).

$2 \mathrm{Zu}$ den wenigen relevanten Quellen zählen vor allem: Barth, K., Systematische Unternehmensführung in den Groß- und Mittelbetrieben des Einzelhandels, Göttingen 1973 (im folgenden zitiert als: Barth,K., Unternehmensführung); Derselbe, Planung, in: Nieschlag, R., Eckardstein, D. (Hrsg.), Der Filialbetrieb als System - das Cornelius Stüssgen Modell, Köln 1972,S.65-88 (im folgenden zitiert als:Barth, K., Planung); Mace,D., Long Range Planning in Retailing, in: Wills,G. (Hrsg.), New Ideas in Retail Management, London 1970,S.21-38; Steffenhagen,H., Marktführer im Zwielicht, in: Moderner Markt 5/1978,S.22-32 (im folgenden zitiert als: Steffenhagen,H., Marktführer). Hier sind auch einige praxisorientierte Beiträge zu nennen,z.B.: BAG (Hrsg.), Unternehmensplanung im Einzelhandel,Köln 1977; Hartmann, K. , Die Unternehmensplanung - eine Kette von Fehlprognosen? in: ZO 1/1979, S.2-4; 0.V., Sear's New 5- Year Plan. To Serve Middle America, in: Kotler,P., Cox,K., Marketing Management, A Reader, Englewood Cliffs,N.J.,1980, S. 248-256. 
um so mehr erstaunen, je detaillierter man die vergangenen, relevanten aktuellen und zukünftigen Veränderungen interner und externer Art analysiert ${ }^{1}$.

In den vergangenen drei Jahrzehnten haben sich grundlegende Veränderungen im Käuferverhalten vollzogen. Waren die 5oer und 6oer Jahre noch stark vom Nachholbedarf geprägt, in denen das Bedürfnis nach Massenware zum günstigen Preis dominierte, so standen die 7oer Jahre ganz im Zeichen differenzierter Kundenwünsche, die sich sowohl auf die Waren als auch auf die Betriebsformen bezogen. Die wachsende Mobilität der Käufer bewirkte außerdem eine Veränderung der Kaufgewohnheiten bezüglich Ort, Häufigkeit und Einkaufsmenge und trug wesentlich $\mathrm{zu}$ einer insgesamt besseren Markttransparenz bei. Eng mit diesen Verhaltensänderungen ist die Verschiebung der Verbrauchsausgabenstruktur zu sehen. Von 1960 bis 1978 nahmen die Ausgaben für"langlebige Gebrauchsgüter" (insbesondere Möbel und Kraftfahrzeuge) sehr stark zu, wogegen die Ausgaben für "sonstige Ge- und Verbrauchsgüter" (insbesondere Nahrungsund Genußmittel, Bekleidung) stark abnahmen ${ }^{2}$. Prognosen ${ }^{3}$ weisen darauf hin, daß diese Entwicklung sich weiter fort-

$1 \mathrm{Vgl} . \mathrm{zu}$ den folgenden Ausführungen insbesondere Berg,H., Die Herausforderung des sich wandelnden Marktes von morgen, in: Handelsblatt $\mathrm{Nr} .231,9 . / 10$. Dezember 1978, S. 17; Eggert, U., Handel 80: Die Zeit der Dinosaurier, in: ASW 12/1978, S. 90-96; Höller, P., Quantitative oder qualitative Expansion? in: Selbstbedienung-Dynamik im Handel 6/1977, S. 12-14; Meffert, H., Perspektiven des Marketing in den 8oer Jahren, in: DBW 40(1980), Heft 1, S. 59-80 (im folgenden zitiert als: Meffert, H., Perspektiven).

$2 \mathrm{Vgl}$. Langmantel, E., Strukturwandel in der Bundesrepublik, in: Ifo-Schnelldienst $34 / 1979$, S. 12-20, hier S. 12 .

3 Vgl. z.B. Prognos AG (Hrsg.), Prognos-Report Nr. 19, Zürich 1979 . 
setzen wird und damit grundsätzliche Anpassungen des Handels im Hinblick auf die Branchenwahl bzw. Sortimentsstruktur notwendig werden. Darüber hinaus werden die rückläufige Bevölkerungsentwicklung und die gleichzeitig wachsende Bedeutung der älteren Konsumenten weitreichende Auswirkungen auf den Handel haben ${ }^{1}$.

Die bisher aufgezeigten Aspekte sind maßgeblich für die Entstehung neuer Betriebsformen verantwortlich. Insbesondere Verbrauchermärkte und SB-Warenhäuser ${ }^{2}$, die durch eine "zeitgemäße" Marktbearbeitung (preisaggressiv, großflächig, Selbstbedienung, am Standrand gelegen ...) den geänderten Kundenwünschen entgegen kamen, gelten als Initiator eines Strukturwandels, der sich in einem starken Rückgang der Zahl der Handelsbetriebe, durch Konzentrationstendenzen ${ }^{3}$ und durch eine starke Verschiebung der Betriebsformen-Marktanteile $e^{4}$ kennzeichnen läßt.

Angesichts der immer häufigeren konjunkturell und strukturell bedingten Schwankungen im Wirtschaftsablauf mu $\beta$ jede Handelsunternehmung zur Sicherung ihres Markterfolges der langfristigen Bindung ihrer Käufer besondere Aufmerksamkeit schenken. Hierzu bedarf es einer klaren Pro-

1 Vgl. hierzu z.B. Buttler,G., Bevölkerungsrückgang in der Bundesrepublik, Ausmaß und Konsequenzen, Köln 1979, S. $167 \mathrm{ff.;}$ Jägeler, F.J., Alle Branchen sind betroffen, in: Manager Magazin 5/1979, S. 74-83.

2 Zur begrifflichen Abgrenzung vgl.: Arbeitsausschuß für Begriffsdefinitionen der Kommission zur Förderung der handels- und absatzwirtschaftlichen Forschung (Hrsg.), Katalog E., Begriffsdefinitionen aus der Handels- und $\mathrm{Ab}-$ satzwirtschaft, 2. Ausgabe, München 1975, S. 21 (im folgenden zitiert als: Arbeitsausschuß Katalog-Kommission). Im folgenden werden beide Betriebsformen synonym betrachtet.

3 Vgl. hierzu z.B. Eggert, U., a.a.O., S. 92 f..

$4 \mathrm{Vgl}$. hierzu die Prognose des Ifo-Instituts bei Batzer, E., Greipl,E., Vor weiteren Marktanteilsverschiebungen im Einzelhandel, in: Ifo-Schnelldienst, 33. Jg., Heft 25 , 4. September 1980, S. 3-8. 
filierung und Akzentuierung der Unternehmung innerhalb der eigenen und gegenüber den übrigen Betriebsformen, die trotz aller situativ bedingten, kurzfristigen Aktivitäten langfristig orientiert sein müssen. Nicht zuletzt muß auch der Handel mit den Problemen der erhöhten Unsicherheit ${ }^{1}$ über Umweltentwicklungen (Einkommen, Wachstumsraten, Wettbewerb, Wirtschaftspolitik) und Unternehmensentwicklungen (Kapitalstruktur, Personalprobleme) fertig werden.

Innerhalb der Großbetriebsformen ${ }^{2}$ des Einzelhandels wirken sich diese Veränderungen in der Umwelt (Käuferverhalten, Strukturwandel usw.) insbesondere auf die Betriebsform Warenhaus aus, was sie in den Mittelpunkt des Interesses rücken $1 \ddot{B} \beta t^{3}$.

1 In Analogie zu Albach, H., Unsicherheit, a.a.0., S. 702.

2 Hierunter versteht man pauschal jene Betriebsformen mit einem Umsatz von über 5o Miliionen DM; vgl. Falk, B.R., Wolf, J., Handelsbetriebslehre, 5. Aufl., München 1979, S. 21 ff.. Vgl, auch die ausführliche Definition bei Conrads, B., Großbetriebsformen des Einzelhandels, in: Handlexikon für Handel und Absatz, hrsg. v. Falk, B., Wolf, J., München 1979, S. 242245 .

3 Hierfür sprechen auch die zahlreichen Beiträge insbesondere populärwissenschaftlicher Art. Vgl. Berg, H., a.a.O., S. 17; Fehr, G., Warenhauskonzerne nach einem Jahrzehnt der Umstrukturierung, in: Rationeller Handel, 3/1980, S. 3o-32; Krüger, H. , Warenhäuser mit neuer Philosophie, in: Markenartikel 2/1979, S. 62-67; Jeske, J.J., Der Verlust der Mitte, in: FAZ Nr. 34, 11.2.1978, S. 15. Vgl. zu primär wissenschaftlichen Ausführungen z.B. Steffenhagen, H. , Marktführer, a.a.o., s. 22-32. 


\subsection{Strategische Marketingplanung in Warenhäusern}

\subsection{Warenhäuser im Spannungsfeld der Betriebsformen}

Generell lassen sich Warenhäuser als abteilungsweise organisierte Großbetriebe kennzeichnen, die Waren aus zahlreichen Branchen bzw. Warengruppen anbieten, mit einem weit verzweigten Filialnetz ausgestattet sind und vornehmlich innerstädtische standorte einnehmen ${ }^{1}$. Mit den vier Warenhauskonzernen Hertie, Horten, Karstadt und Kaufhof $^{2}$ weist der Warenhausmarkt in der Bundesrepublik Deutschland eine oligopolistische struktur auf.

Am Nachkriegsboom in der Bundesrepublik Deutschland partizipierten die Warenhäuser überdurchschnittlich. Der Marktanteil der Warenhäuser am gesamten Einzelhandelsumsatz stieg infolgedessen ständig, von ca. 3\% im Jahre 1949 auf bis $10,5 \%$ in $1974^{3}$. In den folgenden Jahren sank der Marktanteil - entgegen allen Prognosen - auf unter $98(8,98$ in 1979) 4 . Vor dem Hintergrund dieser Entwicklung stellt sich die Frage nach den Gründen sowie nach der grundsätzlichen Richtigkeit der Marktbearbeitungs-Konzeption in der veränderten Situation.

Angesichts des Nachholbedarfs der breiten Massen nach dem zweiten Weltkrieg realisierten die Warenhäuser zunächst

$1 \mathrm{Zu}$ Fassungen des Warenhausbegriffs vgl. z.B. Althoff, T., Waren- und Kaufhaus, in: Tietz, B. (Hrsg.), Handwörterbuch der Absatzwirtschaft, Enzyklopädie der BWL, Bd. IV., Stuttgart 1974, Sp. 2161 ff.; ArbeitsausschuB Katalogkommission, a.a.O., S. 19; Mangold, H.G., Die Absatzpolitik der deutschen Warenhaus-Gesellschaften, Berlin 1971, S. 14 ff..

2 In ihrer Gesamtheit werden diese vier Warenhausgesellschaften in dieser Arbeit als "Warenhäuser" bezeichnet. Die Gleichsetzung der Betriebsform mit diesen vier Gesel1schaften erscheint angesichts eines Gesamtanteils von ca. 90 \& dieser Konzerne am Gesamtumsatz der Warenhäuser (ohne Kleinpreisgeschäfte wie z.B. Woolworth, und ohne die stationären Filialen des Versandhandels) gerechtfertigt.

3 Vgl. Krüger, H., a.a.O., S. 62.

4 Vgl. z.B. Fehr, G., a.a.O., S. 3o f.. 
eine Verhaltensweise, in deren Mittelpunkt das preisgünstige Angebot stand, um der knappen Kaufkraft der Bevölkerung entgegenzukommen. Auf die anspruchsvolleren Wünsche der sich entwickelnden Wohlstandsgesellschaft reagierte das Warenhaus-Management mit der Anhebung des Sortimentsniveaus sowie der Warenqualität und erweiterte das Sortiment um Warengruppen der gehobenen Preisklassen, die bislang nur in Fachgeschäften erhältlich waren ${ }^{1}$. Getreu dem Slogan "tausendfach alles unter einem Dach" boten die führenden Warenhäuser bis zu 160 ooo Artikel in ihren teilweise fachgeschäftsähnlichen Charakter aufweisenden Abteilungen. Durch diese Neuorientierung entstand jedoch eine Lücke in den unteren Preislagen, in die gegen Ende der 6oer Jahre neue Betriebsformen wie Discounter, Verbrauchermärkte und SB-Warenhäuser stießen.

Der Markteintritt dieser Vertriebsformen wurde noch durch einige wichtige allgemeine Veränderungen gefördert, insbesondere den ansteigenden Motorisierungsgrad, die Verlagerung des Wohnbereichs aus der City an die Peripherie sowie die zunehmenden Verkehrsprobleme in den Innenstadtbereichen, den klassischen Standorten der Warenhäuser. Ihre Erfolge lösten einen bis dahin noch nicht gekannten Strukturwandel im Handel aus, der in einer stark veränderten Betriebsformenstruktur seinen Niederschlag fand. Waren die Verbrauchermärkte und SB-Warenhäuser im Jahre 1965 mit einem Marktanteil von 0,4 \& noch völlig unbedeutend, so stieg ihr Marktanteil insgesamt auf über 11 \& in $1979^{2}$, womit sie die klassischen Warenhäuser überrundeten. Die Warenhäuser waren jedoch nicht als einzige Betriebsform vom Strukturwandel betroffen. Insbesondere der Fachhandel wurde durch den Konkurrenzkampf zu einer aktiven Preispolitik gezwungen, die den Abstand zu den Warenhäusern verringerte. Damit entschied sich der Kunde in der

1 Vgl. Jeske, G., a.a.O., s. 15.

2 Vgl. Krüger, H., a.a.O., S. 64. 
Wahlsituation leichter für das Fachgeschäft, weil er bei den problemvollen und hochwertigen waren die individuelle Beratung erwartete.

Durch diese Entwicklungen gerieten die Warenhäuser zwischen zwei Fronten, die auf der einen Seite durch die Verbrauchermärkte und SB-Warenhäuser im Bereich der billigen problemlosen Güter sowie den Artikeln des täglichen Bedarfs und auf der anderen Seite durch die Fachgeschäfte im Bereich der teuren problemvollen und individuellen Güter gehalten werden. Angesichts dieser Tatsache ist es berechtigt, vom "Verlust der Mitte" ${ }^{1}$ für Warenhäuser zu sprechen. Offensichtlich ist es den Warenhäusern nicht gelungen, ihr Profil ${ }^{2}$ in ausreichendem Maße deutlich zu machen ${ }^{3}$. Als Beweis hierfür wird heute auch übereinstimmend die falsche Reaktion der Warenhausleitungen angesehen. Als die neuen Betriebsformen als ernstzunehmende Konkurrenten spürbar wurden, reagierte man mit einer erheblichen Verkaufsflächenexpansion, wobei entgegen der bis dahin verfolgten Standortpolitik auch kleinste Filialen in der Peripherie der Großstädte gegründet wurden, sowie mit verstärkten Rationalisierungsbemühungen sowohl im Personal- als auch im Sortimentsbereich. Durch diese Anpassungsmaßnahmen in Richtung auf das Konzept der "Billigkonkurrenten" verlor man einen wesentlichen Teil der bisherigen Käufer ${ }^{4}$.

Aufgrund dieser Erfahrung bemühen sich die Warenhäuser seit mehreren Jahren in verstärktem Maße darum, ihre Häuser im Bereich der gehobenen Mitte zu profilieren und damit

1 Diesen Begriff prägte Jeske. Vgl. Jeske, J.J., a.a.O., S. 15 .

2 Vgl. hierzu z.B. Hebbering, B., Profil im Verkauf, Ein Warenhaus arbeitet an seiner Identität, in: Moderner Markt, 3/1979, S. 23-27.

3 Vgl. Steffenhagen, H., Marktführer, a.a.o., S. 28.

$4 \mathrm{Vgl}$. ebenda. 
einen Teil des verlorenen Bodens wieder zurückzugewinnen. Hierbei gehen die vier großen deutschen Warenhauskonzerne im Detail betrachtet zwar unterschiedlich vor ${ }^{1}$, die generelle Zielsetzung der Wiedererlangung der starken Marktstellung ist jedoch bei allen gleich. Angesichts der zunehmenden Wettbewerbsintensität im Handel wird sich dieses ziel allerdings langfristig nur unter dem Primat einer marktorientierten Unternehmensführung realisieren lassen. Hieraus wird unmittelbar die Notwendigkeit einer strategischen Marketingplanung in Warenhäusern deutlich. Ihre Realisierung setzt die Anpassung der für Herstellerunternehmungen entwickelten Planungsmethoden auf die besondere Situation der Warenhäuser voraus.

1.22 Gegenstand und Besonderheiten der strategischen Marketingplanung in Warenhäusern

Die strategische Marketingplanung erfordert in einem ersten Schritt die situationsadäquate Festlegung der Planungsobjekte. Dem im Herstellerbereich primär intensivierenden Objektbereich Produkt bzw. Produktprogramm stand im Handel bislang aufgrund seiner Aufgaben der Objektbereich "Ware" gegenüber. Angesichts der skizzierten Veränderungen situativer Rahmenbedingungen und der Notwendigkeit der lokalen Kundenorientierung rückt darüber hinaus zunehmend der Objektbereich "Filiale" in den Vordergrund, da nur erfolgreiches Marketing am einzelnen Standort den Erfolg des Warenhauses als Ganzes bestimmt.

1 Vgl. hierzu im Detail z.B.: Deuss, W., Die Deutschen sind keine Konsummuffel, in: Der Spiegel, 13. Juni 1978, S. 36-41; Freese, G. , Die Verkäufer kommen zurück, wie die großen Warenhäuser aus der Flaute herauskommen wollen, in: Die Zeit, $\mathrm{Nr} .23,27.5 .1977, \mathrm{~S} .18$; Krüger, M., a.a.O., S. 66 f.; 0.V., Wachstum durch Lebensqualität, in: Wirtschaftswoche Nr. 39, 22.9.1978, S. 16-24; O.V., Warenhäuser 77: Zu Euphorie besteht kein Anlaß, in: Rationeller Handel, August 1978, S. 41-45. 
Somit ergibt sich für Warenhäuser die Notwendigkeit, die spezifischen Probleme z.B. des Aggregationsgrades, der Zielgruppenadäquanz der Konkurrenzbeziehungen und des Marketinginstrumente-Einsatzes jeweils für beide Planungsobjekte differenziert zu bearbeiten. Diese isolierte Betrachtung der Planungsobjekte bedarf aufgrund der starken Interdependenzen (z.B. bei den Zielgruppen, Marketinginstrumenten und Ressourcen) anschließend der simultanen Planung bzw. der Integration und Koordination aus gesamtunternehmensbezogener sicht ${ }^{1}$.

Um bereits bei der isolierten Planung von Waren und Filialen einen möglichst hohen Abstimmungsgrad zu erreichen, sind zunächst Rahmenentscheidungen $z u$ treffen, die den Planungsrahmen abstecken. Diese umfassen die Festlegung der Ziele und der grundlegenden Positionierung eines Warenhauses gegenüber den Käufersegmenten und den Konkurrenten $^{2}$. Die Zielbildung beinhaltet die konkrete Definition der Marketingziele in Warenhäusern nach Inhalt, Ausmaß Zeit- und Segmentbezug. Sie sind im Zielsystem zu dokumentieren und beschreiben den zukünftigen Zustand des Warenhauses, der als Ausdruck der politischen Willensbildung als anstrebenswert erachtet wird. In ihrer Ausgestaltung weisen sie unmittelbar auf die Positionierungsentscheidung hin.

1 Die "Integration" bezeichnet jene planerischen Aktivitäten, die eine vertikale Abstimmung von hierarchisch über- und untergeordneten Plänen, Zielen oder Strategien bewirken. Die Koordination bezieht sich auf die horizontale Abstimmung unter hierarchisch gleichrangigen Planungsträgern bzw. -instanzen. Vgl. zu diesen Begriffen Wild, J., Grundlagen der Unternehmensplanung, Reinbek 1974, S. $161 \mathrm{f}$. und S. $191 \mathrm{ff}$.

2 Damit wird die Positionierung eines Warenhauses, d.h. seine Stellung am Markt explizit durch die Dimensionen "Kunde" und "Konkurrenz" bestimmt. Ahnlich definiert Kotler,der jedoch primär auf den Konkurrenzaspekt abstellt und die Kundendimension implizit berücksichtigt. Vgl. Kotler, P., Marketing Management, a.a.o., S. $272 \mathrm{ff.}$ 
Die zunehmende Differenzierung der Kundenbedürfnisse und die daraus resultierende Notwendigkeit, diese an den Anfang jeglicher Marktbearbeitungsstrategien zu stellen, macht es im Rahmen der strategischen Marketingplanung für Warenhäuser unabdingbar ${ }^{1}$, ihre Position gegenüber der Käuferschaft durch die Festlegung zu bearbeitender Zielgruppen $\mathrm{zu}$ bestimmen. Dies impliziert unmittelbar auch die Festlegung der Verhaltensweisen gegenüber der Konkurrenz (= Konkurrenzstrategie), da diese u.U. gleiche oder ähnliche Ziele verfolgt, gleiche oder ähnliche Zielgruppen bearbeitet und somit "... versucht, durch Gegenaktionen die eigenen Marktvorhaben zu stören" ${ }^{2}$.

Mit den Entscheidungen über die Ziele und Positionierung des Warenhauses sind die Voraussetzungen für die Entwicklung von Marketingstrategien für die Planungsobjekte geschaffen. Aufgrund der nahezu unbegrenzten Möglichkeiten, Marketingstrategien zu formulieren, erscheint es sinnvoll, der Entwicklung von Marketingstrategien ein Grundraster zugrunde zu legen, das einerseits dem generellen Marketingverständnis als marktorientierter Führung und andererseits den realen strategischen Marketingproblemen der Warenhäuser Rechnung trägt. Zu diesem Zweck wird zwischen Basis- und Marketing-Instrumentalstrategien differenziert $^{3}$. Die Basisstrategien legen als Ausdruck der globa-

1 Vgl. hierzu u.a. Ahlert, D., Marketing, a.a.O., S. 41 ff.; derselbe, Probleme der Abnehmerselektion und der differenzierten Absatzpolitik auf der Grundlage der segmentierenden Markterfassung, in: Der Markt 1973, Nr. 4, $\mathrm{S} .103 \mathrm{ff} . ;$ Meffert, H., Marketing. Einführung in die Absatzpolitik, 5. Aufl., Wiesbaden 1980, S. $213 \mathrm{ff}$. (im folgenden zitiert als: Meffert, H., Marketing).

2 Pümpin, C.B., a.a.O., S. 34 .

$3 \mathrm{Vgl}$. zu einer ähnlichen systematisierung von Marketingstrategien: Hoffmann, K., Wolff, V., Zur Systematik von Absatzstrategien als Grundlage langfristig wirkender Entscheidungen im Absatzbereich, in: JAVF, 23. Jg., Heft 2/1977, S. 161-175, hier S. 163 ff.; Meffert, H., Strategische Planung, a.a.0., s. 90. 
len Vorgehensweise die "strategischen Stoßrichtungen" eines Warenhauses fest und stellen damit das logische Bindeglied zwischen den Rahmenentscheidungen und dem strategischen Einsatz der Marketinginstrumente dar. Ihre Konkretisierung erfolgt durch die Festlegung des Aktivitätsniveaus der Marketinginstrumente im Rahmen der Instrumentalstrategien. Sie umfassen demnach die grundlegenden Verhaltensweisen in den Bereichen der Produkt- und Sortimentspolitik, der Distributions- Kontrahierungs- und Kommunikationspolitik ${ }^{1}$.

In der Realität der strategischen Planung von Warenhäusern sind die aufgezeigten Einzelentscheidungen weder klar voneinander zu trennen noch sind sie in ihrer Reihenfolge fest determiniert. Dies berührt die zentrale Problematik einer prozessualen Betrachtung der strategischen Planung. Einerseits erfordert die Komplexität strategischer Planungsprobleme aufgrund der begrenzten menschlichen Problemlösungsfähigkeiten eine (idealtypische) Aufteilung des Planungsgeschehens in überschaubare Teilphasen (vgl. auch Abbildung 1). Andererseits wird dadurch der Anschein einer ausgeprägten logisch abfolgenden Sukzessivität erweckt, die weder zwingend gute Lösungen hervorbringt noch in der Realität vorzufinden ist.

1 Damit wird einer heute weitgehend anerkannten systematisierung der Marketinginstrumente gefolgt. Vgl. hierzu Meffert, H., Marketing, a.a.O., S. 82 ff... Eine abweichende Systematik stellt z.B. Ahlert vor. Er differenziert zwischen Abnehmerselektionspolitik und $\mathrm{Ab}-$ nehmerakquisitionspolitik und gliedert letztere in $\mathrm{Ab}-$ satzprogramm-, Preis-, Absatzkonditionen-, Absatzkommunikationspolitik. Vgl. Ahlert, D., Marketing, a.a.O., S. $49 \mathrm{ff.}$ 
So liegt z.B. in der (Neu-) Konzeption der strategischen Geschäftseinheiten ein beträchtliches strategisches Innovationspotential - ihre endgültige Bildung ist jedoch erst dann möglich, wenn klare ziele und Strategien entwickelt sind. Um diese entwickeln zu können, sind jedoch grobe Vorstellungen über die strategischen Geschäftseinheiten notwendig.

Ähnliche Aussagen gelten für jede Teilphase des Planungsprozesses. Damit erscheint zwar die idealtypische Aufteilung des Planungsgeschehens in einzelne Teilphasen zur besseren Durchdringung und Strukturierung der strategischen Probleme eines Warenhauses gerechtfertigt, jedoch muß stets berücksichtigt werden, daß es sich um "interdependente Planungsvorgänge handelt, die in einem dynamischen Rückkoppelungsprozeß ohne Anfang und Ende eingebunden sind ${ }^{1}$.

Berücksichtigt man neben der Festlegung des Planungsobjektes, den Rahmenentscheidungen und der Ableitung von Strategien zusätzlich die Informationsphase, in deren Rahmen die relevanten internen und externen Informationen gewonnen werden, sowie die Realisations-, und Kontrollphase,

1 Zahn, E., a.a.O., S. 229. Dies betrifft vor allem die Ziele, die Positionierung und die Strategiefindung. Diese drei Bereiche sind in einem iterativen Proze $\beta$ festzulegen. 
die die Umsetzung, die Ermittlung von Abweichungen urd ggf. Anpassungsmaßnahmen beinhaltet, so läßt sich der Prozeß der strategischen Marketingplanung in Warenhäusern wie in Abbildung 1 darstellen ${ }^{1}$.

Neben der prozessualen Betrachtung sind für die strategische Marketingplanung von Warenhäusern insbesondere noch die Fragen nach den Planungsträgern und der Planungsmethoden zu klären.

Als Träger der strategischen Marketingplanung in Warenhäusern fungiert der Vorstand, unterstützt durch einen zentralen Marketingstab. Ausgestattet mit den notwendigen Kompetenzen zur Übernahme und Delegation der strategischen Planungsaufgaben stüzt er sich bei seinen Entscheidungen auf die Erfahrungen und spezifischen Kenntnisse der Bereichsleiter "Ware" und "Filialen", denen entsprechende Mitspracherechte bei der strategischen Marketingplanung eingeräumt werden.

Im Rahmen der Durchsetzung der strategischen Entscheidungen stellt sich für Warenhäuser insbesondere das Problem der Verteilung der Planungskompetenz zwischen der Zentrale und den dezentralen Organisationseinheiten. Es besteht darin, daß dezentrale Entscheidungen über die einzelnen Waren oder Filialen die spezifische Marktsituation

1 Dieser Phaseneinteilung wird heute in der Planungs-Literatur überwiegend gefolgt. Vgl. hierzu für die industrielle Planung z.B. Gälweiler, A., Unternehmensplanung, a.a.O., S. 172 ff.; Töpfer, A., a.a.O., S. 80 ff.; Wild, J., Grundlagen, a.a.O., S. 32 ff.. Eine ähnliche Phasenaufteilung wird üblicherweise auch für handelsspezifische Planungsprobleme gewählt. Vgl. hierzu z.B. Bearchell, C.A., Retailing: A Professional Approach, New York usw. 1975, S. 54 ff.; Berman, B., Evans, J.R., Retail Management. A Strategy Approach, New York 1979, S. 90 ff.; Davidson, W.R., Doody, A.F., Sweeney, D.J., Retailing Management, 4. Aufl., New York 1975, S. 83 ff.. 


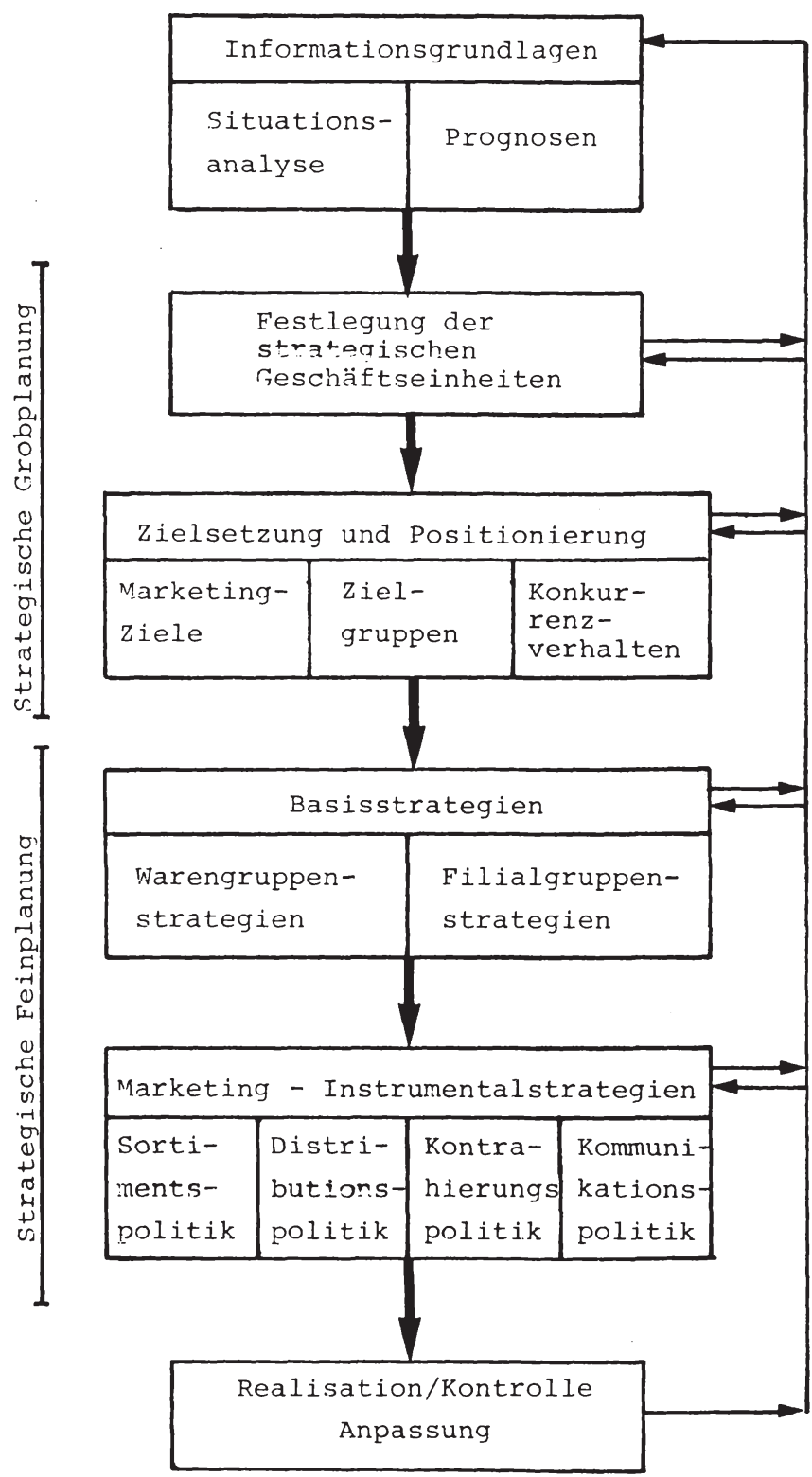

Abb. 1: Prozeß der strategischen Marketingplanung in Warenhäusern Friedrich Wehrle - 978-3-631-75094-0 
$z u$ berücksichtigen haben und damit mehr oder weniger stark von zentralen Entscheidungen abweichen, die sich eher an den "durchschnittlichen" Marktgegebenheiten zu orientieren haben. Aufgrund derartiger Divergenzen muß die Warenhausleitung eine Planungssystematik entwickeln, die die übergeordneten, zentralen Belange mit den dezentralen Vorstellungen in Einklang bringt und damit die Realisierung der strategischen Planung auch in den dezentralen Einheiten gewährleistet. Vor dem Hintergrund der aktuellen "Warenhauskrise" hat dieser Problemkreis besondere Beachtung gefunden ${ }^{1}$.

Die vielschichtigen Probleme bei der Ableitung von Marketingstrategien in Warenhäusern rücken die Frage nach planungsmethoden im Sinne globaler Denkkonzepte in den Vordergrund, die die Komplexität des strategischen Problems zu reduzieren, die Vielfalt der Einzelaspekte zu strukturieren sowie überschaubarer und handhabbar $\mathrm{zu}$ gestalten vermögen ${ }^{2}$. Aufgrund der teilweise schwierig $z u$ qualifizierenden und in mathematische strukturen faßbaren Probleme gewinnen heuristische Planungsmetho-

1 Vgl. hierzu z.B. Alberts, K., Sortimentsfight statt Flächenklotzerei, in: ASW 9/1979, S. 6-8; Käckenhoff, U., Wieder Schwung im Laden, in: Manager Magazin, 11/1979, S. 188-195, hier S. 192; Steffenhagen, H., Marktführer, a.a.O., S. $28 \mathrm{ff}$.; Derselbe, Zukunft fordert Strategie, in: SB-Warenhaus 4/1979 (Teil I), S. 6-10 und 5/1979 (Teil II), S. 6-7 (im folcenden zitiert als: Steffenhagen, H., Strategie, Teil I und Teil II); vgl. hierzu auch die empirische Untersuchung von Herder. Vgl. Herder, H., Filialunternehmen: Alle Macht der Zentrale?, in: Rationeller Handel, 4/1979, S. 2-8; derselbe, Filialorganisation: Optimierung mit Kompromissen, in: Rationeller Handel, 5/1979, S. 8-13; derselbe, Wie zentralistisch ist mein Betrieb?, in: Rationeller Handel, 6/1979, S. 33-39; derselbe, Determinanten der (De-) Zentralisation, in: Rationeller Handel 1/1980, S. 2-6 (im folgenden wird die gesamte Aufsatzserie zitiert als: Herder, H., Zentralisation, Teil I-IV).

2 Vgl. Roventa, P., a.a.O., S. 76. 
den ${ }^{1}$ zunehmend an Bedeutung, wobei die Portfolio-Methode im Mittelpunkt der aktuellen Diskussion steht ${ }^{2}$.

1 Ungeachtet der uneinheitlichen Begriffsfassung kann eine Planungs- bzw. Entscheidungsmethode dann als heuristisch bezeichnet werden, wenn die eingesetzten Verfahrensregeln im Durchschnitt den Zeitaufwand zur Lösung verringern und dadurch Teile der potentiellen Lösung nicht erfassen, sie nur für eine bestimmte Klasse von Problemen gilt und keine Lösungsgarantie gegeben werden kann (in Anlehnung an Klein, H., Heuristische Entscheidungsmodelle, Wiesbaden 1971, S. 36 ;

Scheuch, F., Heuristische Entscheidungsprozesse in der Produktpolitik, Berlin 1977, S. $33 \mathrm{f} .$. Vgl. als weitere grundlegenden Beiträge z.B. Frese, E., Heuristische Entscheidungsstrategien der Unternehmensführung, in: $\mathrm{Z}_{\mathrm{fbF}}$ 1971, S. 283-306; Imboden, C., Leibundgut, A., Siegenthaler, P., Klassifikation heuristischer Prinzipien, in: DU 32 (1978), S. 295-330; Streim, H., Heuristische Lösungsverfahren. Versuch einer Begriffsklärung, in: Zeitschrift für Operations Research, Bd. 19 (1975), S. 143-162; Ulrich, W., Einführung in die heuristischen Methoden des Problemlösens, in: Wisu 6/1976, S.63-68.

2 Als weitere, unter heuristischen Gesichtspunkten einerseits und der praktischen Bedeutung andererseits aktuell diskutierte (heuristische) strategische Planungsmethoden sind z.B. die Gap-Analyse, die Lebenszyklusanalyse sowie die Misfit-Analyse und die Diskontinuitätenplanung zu nennen. Vgl. zur Gap-Analyse z.B. Ansoff, H.I., Management-Strategie, a.a.O., S. 125 ff.; Kami, M.J., Gap-Analysis, Key to Super Growth, in: LRP 4/ 1969, S. 44-47; Kreikebaum, H., Die Lückenanalyse als Voraussetzung der Unternehmensplanung, in: Interne Revision, 1/1973, S. 17-26; Meffert, H., Strategische Planung, a.a.0., S. 95; Roventa, P., a.a.O., S. 77-84; Thanheiser, H., Patel, P., Strategische Planung in diversifizierten deutschen Unternehmen, Wiesbaden 1977, S. 56 f.; Zahn, E., a.a.O., S. 248 ff..

Vgl. zur Lebenszyklus-Analyse z.B. Ahlert, D., Marketing, a.a.O., S. 96 f.; Clifford, D.K., Managing the Product Life Cycle, in: Kotler, P., Cox, K., Marketing Management and Strategy. A Reader, Englewood Cliffs, N.J.,1980, S. 175-181; Enis, B.M., La Garce, R., Prell, A.E., Extending the Product Life Cycle, in: Business Horizons, Vol. 20, June 1977, S. 46-56; Freudemann, H., Planung neuer Produkte, Stuttgart 1965; Hoffmann, K., Der Produktlebenszyklus, Freiburg 1972; Kotler, P., Marketing Management, a.a.O., S. $289 \mathrm{ff}$; Levitt, T., Exploit the Product Life Cycle, in: HBR, Vol. 43, Nov.-Dec. 1965, S. 81-94; Meffert, H., Interpretation und Aussagewert des Produktlebenszyklus-Konzepts, in: Neuere Ansätze der Marketingtheorie, Festschrift zum 80 . Geburtstag von Otto Schnutenhans , Hrsg.:Hammann, P., Kroeber-Riel, W. , Meyer, C.W., Berlin 1974, S. o4-134 (im folgenden zitiert als: Meffert, H., Produktlebenszyklus; Derselbe, Marketing, a.a.O., S. 339 ff.; Weber, J.A., Planning Corporate 
1.3 Bedeutung der Portfolio-Methode für die strategische Marketingplanung in Warenhäusern

Die Portfolio-Methode ${ }^{1}$ (Portfolio-Analyse, PortfolioManagement) ist ein relativ neues Denkschema der strategischen Unternehmens- und Marketingplanung. In Anlehnung an das Portfolio von Wertpapieren wird hierbei eine Unternehmung als Gesamtheit von einzelnen Produkten, Produktlinien oder - in der Terminologie der Portfolio-Methode "strategischen Geschäftseinheiten"2 gesehen. Es gilt,eine Ausgewogenheit im Gesamt-Portfolio der Unternehmung herzustellen. Diese ist erreicht, wenn eine Portfolio-Konstellation realisiert ist, die einerseits die Zielerreichung sicherstellt und andererseits durch eine Mischung von finanzmittel-freisetzenden und finanzmittel-verbrauchenden strategischen Geschäftseinheiten gekennzeichnet ist, bei der die Liquidität des Unternehmens stets gewährleistet ist.

2 (Fortsetzung von S. 23)

Growth with Inverted Product Life Cycles, in: LRP, Oct. 1976, S. 12-29; Wesner, E., Die Planung von Marketingstrategien auf der Grundlage des Modells des Produktlebenszyklus, Diss. Berlin 1977.

Zur Misfit-Analyse und Diskontinuitätenplanung vgl. Ansoff, H.I., Hayes, R.L., a.a.O., S. 1 ff.; Ansoff, H.I., Declerck, R.P., Hayes, R.L., From Strategic Planning to Strategic Management, in: Ansoff, H.I., Declerck, R.P., Hayes, R.L., (Hrsg.), From Strategic Planning to Strategic Management, London 1976, S. 39-78, hier S. 39 ff..

1 Einen guten Überblick geben z.B. Abell, D.F., Hammond, J.S., Strategic Market Planning, Englewood Cliffs, N.J., 1979 , S. $173 \mathrm{ff.;}$ Albach, H., Unsicherheit, a.a.O.,s.7o2 ff.; Ansoff, H.I., Leontiades, J.C., Strategic Portfolio Management, EIASM Working Paper 76-16, Brüssel 1976; Dunst,K., Strategisches Portfolio-Management, Berlin/New York 1979, insbesondere S. $89 \mathrm{ff.;}$ Hinterhuber, H., a.a.O., insbes. S. $89 \mathrm{ff.;}$ Jünger, E.P., Globalitätsgrade und Verfahren der Produktionsprogrammplanung im Rahmen der hierarchischen Unternehmensplanung, Diss. Münster 1980,S.120-177;Szyperski, N., Winand,U., Strategisches Portfolio-Management;Konzept und Instrumentarium, in: $\mathrm{Z} \mathrm{fbF} \mathrm{1978,} \mathrm{S.} \mathrm{123-132;} \mathrm{Wittek,}$ B.F., Strategische Unternehmensführung bei Diversifikation, Berlin/New York 1980, S. $131 \mathrm{ff..}$

2 Ihre materielle Ausgestaltung für die strategische Marketingplanung von Warenhäusern ist Gegenstand des 2.Kapitels. 
Bei einer Anwendung in Warenhäusern besteht das Anliegen des Portfolio-Konzeptes darin, die Chancen und Risiken von Planungsobjekten (strategischen Geschäftseinheiten) durch ein System von relevanten Bestimmungsfaktoren ${ }^{1}$ zum Ausdruck zu bringen. Gruppiert man diese Bestimmungsfaktoren in zwei Hauptdimensionen, so läßt sich unabhängig von ihrer konkreten Ausprägung eine zweidimensionale Matrix aufstellen, in die sich die Planungsobjekte (strategische Geschäftseinheiten) des Warenhauses positionieren lassen. Hierbei wird die eine Achsendimension in der Regel von unternehmensbezogenen Faktoren bestimmt, wie z.B. Warenqualität, Sortimentsattraktivität, Marketingpotential, Ertragskraft. Die zweite Dimension spiegelt dagegen marktbezogene Faktoren wider, wie z.B. Marktvolumen, Produktlebenszyklusstadium oder Marktwachstum.

Ist die Auswahl der relevanten Faktoren ${ }^{1}$ durchgeführt, werden die verschiedenen Planungsobjekte beurteilt und in der Matrix positioniert (vgl. Abbildung 2). Die sich zeigenden Unausgewogenheiten in der Matrix sind als Ausgangspunkt und Stimulanz für die Suche nach Strategien zur Herbeifürung der Ausgewogenheit des Portfolios zu interpretieren. Dabei liefern die in der Matrix aufgezeigten Positionen bereits wesentliche Hinweise auf $\mathrm{zu}$ ergreifende Basis-Strategien (in der Terminologie der PortfolioAnalyse "Normstrategien" genannt) in Warenhäusern, die $\mathrm{zu}$ einer Verbesserung der Position im Sinne einer insgesamt größeren Ausgewogenheit des Portfolios führen. Diese Basisstrategien lassen sich in ihrer allgemeinsten Form als Investitions-, Desinvestitions- oder Selektionsstra-

1 Die Vielzahl denkbarer Einflußfaktoren hat zu einer großen Anzahl von Portfolio-Varianten geführt, die sich jedoch nicht im Grundgedanken, sondern lediglich in den Achsenbezeichnungen den erstellten Matrizen und damit den entwickelten Normstrategien unterscheiden. VGl. den guten Überblick z.B. bei Roventa, P., a.a.O., S. $132 \mathrm{ff.}$. 


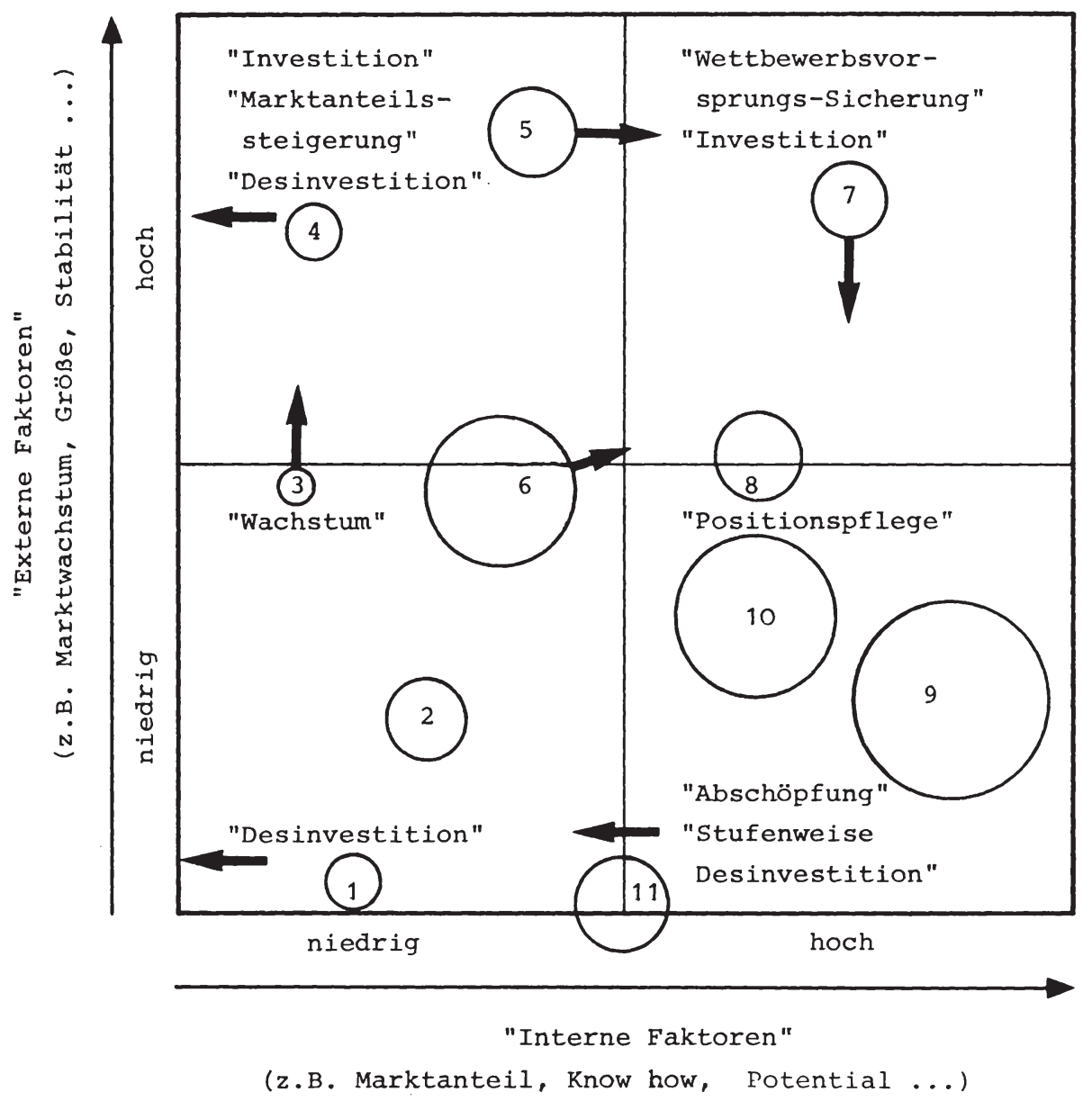

Legende: (1) Die Kreise 1-11 kennzeichnen die strategischen Geschäftseinheiten

(2) Die Größe der Kreise bezeichnet die Umsatzbedeutung

(3) Die Normstrategien sind den einzelnen strategischen Geschäftseinheiten beispielhaft zugeordnet

Abb. 2 : Grundstruktur des Portfolio-Konzeptes 
tegien bezeichnen. Ihre spezifische Ausprägung ist stark von dem zugrundegelegten Einflußfaktorensystem, das die Achsenbezeichnungen determiniert, abhängig.

Bei der Bestimmung der relevanten Einflußfaktoren müssen einerseits die gewinn- und risikowirksamen Faktoren vollständig erfaßt werden, um die Qualität der Planung nicht zu gefährden. Andererseits aber muß eine Reduktion auf wenige wichtige Einflußfaktoren erfolgen, um die Transparenz und Handhabbarkeit des Systems zu wahren ${ }^{1}$. Je nach Anspruchsniveau (Genauigkeitsgrad) der Planung kann diese Reduktion aufgrund von Kreativität, Intuition, Plausibilitätsüberlegungen ${ }^{2}$ oder aufgrund empirischer Untersuchungen oder theoretischer Erkenntnisse durchgeführt werden.

In diesem Zusammenhang ist im Herstellerbereich die Gültigkeit des Erfahrungskurveneffektes als relevantes Kriterium für die Auswahl der Schlüsselfaktoren nachgewiesen worden ${ }^{3}$. Er besagt, daB die Stückkosten in Abhängigkeit von der kumulierten Produktions- und Absatzmenge bedingt durch Erfahrung sinken. Dabei bezieht sich die Erfahrung sowohl auf Kostendegressionseffekte bei der Produktion

1 Vgl. Szyperski, N., Winand, U., a.a.O., S. 125.

2 Insbesondere bei der praktischen Anwendung im Beratungsfall werden die Bestimmungsfaktoren aufgrund von Intuition oder Plausibilitätsüberlegungen der Manager festgelegt. Vgl. hierzu z.B. die unternehmensspezifisch festgelegten Erfolgsfaktoren bei: Hussey, D.W., Portfolio Analysis: Practical Experience with the Directional Policy Matrix, in: LRP Aug.1978,S. 2-8; Robinson, S.J., Hichens, R.E., Wade,D.P., The Directional Policy Matrix - Tool for Strategic Planning, in: LRP, June 1978, S. 8-15.

$3 \mathrm{Vgl}$. zur Darstellung des Effektes und seiner Implikationen für die Planung von Marketingstrategien z.B. Gälweiler, A., Unternehmensplanung, S. 241 ff.; Hedley, B., Strategy and the Business Portfolio, in: LRP, Vol.10, Febr. 1977, S.9-15; Henderson, B.D., Die Erfahrungskurve in der Unternehmensstrategie, Frankfurt/New York 1974; Nagtegaal, H., Wie überlebt mein Unternehmen, Wiesbaden 1975, S. 32 ff; Roventa,P.,a.a.O.,S.132-139; Ziegenbein, K., Der Boston-Effekt, in: ASW 12/1975,S.72-75, insbes.S. 72: Gälweiler, A., Marketingplanung im System einer integrierten Unternehmensplanung, in: Poth, L.(Hrsg.), Marketing Bd. 2, Neuwied 1979, S. $19 \mathrm{ff}$.. 
durch Ferneffekte und Rationalisierungsmaßnahmen als auch auf Erfahrungen im Marketingbereich z.B. im Bereich des persönlichen Verkaufs sowie Synergien in der Werbung und im Vertriebssystem ${ }^{1}$. Der Erfahrungskurveneffekt ist auf Warenhäuser nur bedingt übertragbar. Insbesondere durch die fehlende Produktion liegt ein wesentlicher Bestandteil des Kostensenkungspotentials eines Artikels nicht im Entscheidungsbereich der Warenhausleitung. So lassen sich Erfahrungseffekte bei Warenhäusern primär im Marketingbereich plausibel begründen ${ }^{2}$. Durch steigende Ein- und Verkaufsmengen lassen sich in der Regel günstigere Konditionen erzielen, die den Kalkulationsspielraum der Warenhausleitung erhöhen. Gleichzeitig werden tendenziell kostensenkende Rationalisierungsmaßnahmen im Lager- und Transportwesen erleichtert. Folgt man der Hypothese, daß die Qualifikation und Leistung des Verkaufspersonals wesentlich von der Erfahrung mit bestimmten Artikeln bzw. Artikelgruppen abhängt, so nimmt diese im Zeitablauf (bei gleichen oder ähnlichen Artikeln) zu. Dies kann sowohl zu einer besseren Verkaufsleistung führen, als auch -7.B. durch die fbernahme mehrerer Funktionen ( Beratung, Verkauf, Disposition etc.) - zu unmittelbar kostenwirksamen Effekten führen. Darüber hinaus lassen sich bei Warenhäusern durch die große Zahl der Artikel einerseits sowie die Vielzahl der Verkaufsstellen andererseits synergieeffekte in der Werbung erzielen (z.B. erhöhen zentrale Werbekampagnen die Kundenfrequenz in den einzelnen Häusern).

1 Damit ergibt sich für Unternehmen, die über große akkumulierte Erfahrung verfügen eine relativ zum Wettbewerber günstige Kostenposition, die z.B. bei Preisstrategien, Budgetfragen usw. zentrale Bedeutung erlangt.

2 Empirische Untersuchungen zu diesem Problemkreis sind bislang nicht bekannt. 
Der Erfahrungskurveneffekt weist unmittelbar auf die Bedeutung des Marktanteils ${ }^{1}$ und des Marktwachstums als Schlüsselfaktoren hin. Auch hier gelten die für Industrieunternehmen teilweise empirisch begründeten Auswirkungen für Warenhäuser nur bedingt. Aufgrund von Plausibilitätsüberlegungen läßt sich die These aufstellen, daß mit zunehmendem Marktanteil eines Warenhauses - gemessen z.B. an einem bestimmten Artikel, einer Artikelgruppe oder auch in einer Region - die gesammtelte (Marketing-) Erfahrung wächst und damit ein Kostensenkungspotential gegenüber Konkurrenten mit niedrigeren Marktanteilen aufgebaut wird. Die Relevanz des Marktwachstums als Schlüsselfaktor liegt darin begründet, daß sich in wachsenden Märkten ${ }^{2}$ die absolute Absatzmenge ex definitione vergrößert, Marktanteile leichter zu erringen sind und sich selbst bei gleichbleibendem Marktanteil durch den steigenden Absatz Erfahrungseffekte ergeben.

Die Marktwachstumsrate einer Ware bzw. einer Warengruppe hängt wesentlich von ihrer jeweiligen Phase im Lebenszyklus ab. Dabei wird bei fortschreitendem Lebenszyklus sinkende Wachstumsraten bzw. ein negatives Wachstum in der Verfallsphase angenommen. Trotz der berechtigten Kritik ${ }^{3}$

1 In den letzten Jahren wurden mehrere Ansätze entwickelt, auf der Basis des Marktanteils von Unternehmungen Marketingstrategien $\mathrm{zu}$ entwickeln. Vgl. hierzu z.B. Bloom, P.N., Kotler, P., Strategies for High MarketShare Companies, in: HBR, Vol. 53, Nov.-Dec. 1975, S. 63-72; Fogg, D.C., Planning Gains in Market Share, in: JoM 38 (July 1974), S. 30-38; Hamermesh, R.G., Anderson, M.J., Harris, J.E., Unternehmensstrategien bei niedrigen Marktanteilen, in: Harvard Manager, 3/1979, S. 4o-48.

2 Dabei muß der "Markt"-Begriff nicht unbedingt auf ein Produkt bezogen sein, sondern kann auch aggregierter gefaßt werden, z.B. als Warengruppe oder Branche.

3 Diese setzt insbesondere an den Prämissen an und verweist auf die fehlende, bisher noch nicht nachgewiesene Allgemeingültigkeit, die weder empirisch noch theoretisch nachgewiesene Gesetzmäßigkeit sowie die nicht durchführbare eindeutige Abgrenzung der Phasen. Vgl. zur detaillierten Kritik insbesondere: Dhalla, N.K., Yuspeh, S., Abschied vom Konzept des Produktlebenszyklus (dt. Ufbersetzung), in: Harvard Manager, Heft 1/1980, S. 69-79, hier S. 69 ff.; Meffert, H., Produktlebenszyklus, a.a.O., S. 84 ff.. 
an diesem Konzept ist es in Industrieunternehmungen als strategisches Denkraster weit verbreitet ${ }^{1}$. Dennoch gilt auch hier, daß die Übertragung der Überlegungen auf die einzelnen Artikel bzw. Artikelgruppen bzw. Filialen eines Warenhauses oder auf die Betriebsform Warenhaus als Ganzes problematisch $z u$ sehen sind. Die aus der Literatur bekannten Versuche, die Gültigkeit des Lebenszykluskonzeptes für Betriebsformen als Ganzes zu belegen ${ }^{2}$, sind insgesamt als interessante jedoch keineswegs befriedigende Versuche $\mathrm{zu}$ werten. Insofern ist - wie bei Industrieunternehmungen auch - die Berücksichtigung der Position im Lebenszyklus für warenhausspezifische Problemstellungen zwar möglich, doch müssen stets die begründeten Vorbehalte gegen das Konzept beachtet werden.

Die zentrale Bedeutung dieser Bestimmungsfaktoren belegen auch die Erkenntnisse aus der PIMS-Studie ${ }^{3}$. Aus-

1 Vgl. zum Einsatz des Konzeptes auch Meffert, H., Strategische Planung, a.a.0., S. 96 f..

2 Vgl. hierzu neuere Arbeiten z.B. von Berger, S., Ladenverschleiß (Store-Erosion) - Ein Beitrag zur Theorie des Lebenszyklus von Einzelhandelsgeschäften, Göttingen 1977; Mathieu, G., Lebenszyklus als Entscheidungshilfen für Betriebstypen im Handel, in: Marketing Journal 2/1978, S. 122-128; Moser, D., Neuere Betriebsformen im Einzelhandel, Frankfurt/Zürich 1974; Müller-Hagedorn, L., Bevorzugte Betriebsformen des Einzelhandels und das Lebenszykluskonzept, in: $\mathrm{ZfbF} 30$ (1978), S. 106-124; Davidson, W., Der Lebenszyklus im Einzelhandel, in: Harvard Manager 2/1979, S. 46-54.

$3 \mathrm{Vgl}$. zur Darstellung, den Anwendungsmöglichkeiten und Ergebnissen der PIMS-Studie (Profit Impact of Market Strategies) z.B. Abell, D.F., Hammond, J.S., a.a.O., S. 271 ff.; Buzzell, R.D., Gale, B.T., Sultan, R.G., Market Share - a Key to Profitability, in: HBR, Jan.Febr. 1975, S. 97-106; Neubauer, F.F., Strategien besser absichern, in: Manager Magazin, 12/1979, S. 154-159; derselbe, Wer wird aurch die Rezession besonders hart getroffen?, in: Blick durch die wirtschaft, Nr. 90, 17. April 1980; Roventa, P., a.a.O., S. 114-119; Schöffler, S., Buzzell, R.D., Heany, D.F., Impact of strategic planning on profit performance, in: HBR March-April 1974, S. 137-145; Wittek, B.F., a.a.O., S. $160 \mathrm{ff.}$. 
gehend von der Zielsetzung, den ROI von Unternehmen zu erklären, weist sie zusätzlich die Erklärungsrelevanz weiterer (ca. 26) Faktoren, u.a. der Marketingausgaben, der Unternehmensgröße (gemessen am Umsatz) sowie der Investitionsintensität aus. Je intensiver angesichts der zunehmenden Konkurrenzintensität die Kundenakquisition betrieben werden muß, desto differenzierter und konzeptionell durchdachter müssen die Marktbearbeitungsaktivitäten sein und desto höher ist das Marketingbudget $\mathrm{zu}$ bemessen. Diese Handlungsmöglichkeiten hängen ihrerseits wieder eng mit der Unternehmensgröße zusammen. Je größer das Warenhaus ist, desto gröBer ist das finanzielle Potential und damit die Voraussetzung, entsprechende Marketingbudgets bereitzustellen. Die gleiche úberlegung trifft ebenso für die Investitionsintensität $z u$.

Diese Ausführungen verdeutlichen, daß die Anwendung der Portfolio-Methode auf strategische Problemstellungen der Warenhäuser eine Modifikation insbesondere der Prämissen erforderlich macht, als deren unmittelbare Konsequenz die Schlüsselfaktoren festgelegt werden. Insofern sind die später zu diskutierenden Portfolio-Varianten stets vor dem Hintergrund einer lediglich auf plausiblen Ubberlegungen begründeten Auswahl der Schlüsselfaktoren kritisch zu betrachten ${ }^{1}$. Weiterhin sind im Rahmen einer kritischen Analyse des Aussagegehalts dieser Planungsmethode vorhandene praktische Erfahrungen $z u$ berücksichtigen. So dürfen bei der Portfolio-Methode heute neben ihren zweifellos vorhandenen Vorzügen, z.B. der Verdichtung relevanter Informationen, der Komplexitätsreduktion und Handhabbarmachung des strategischen Problems, der Förderung der Kom-

1 Dabei darf allerdings nicht übersehen werden, da $B$ in der Mehrzahl der Anwendungsfälle in Industrieunternehmungen die Auswahl der Schlüsselfaktoren auch auf der Basis von Plausibilitätsüberlegungen getroffen wird, die sich oftmals lediglich auf Vermutungen des Managements uber die Zusammenhänge zwischen Faktoren stützt. 
munikation zwischen den Entscheidungsträgern, der Verknüpfung interner und externer strategiebestimmender Einflußgrößen einige Schwierigkeiten nicht übersehen werden.

Bei der Abgrenzung und Festlegung der strategischen Geschäftseinheiten entsteht zunächst der Konflikt, daß diese einerseits losgelöst von organisatorischen Zwängen zu bilden sind, daß die auf ihnen basierenden Marketingstrategien dann aber andererseits in der organisation umgesetzt werden müssen. Als weiteres Problemfeld ist die zweckadäquate Dimensionierung der Portfolio-Varianten anzusehen, die bislang für die Warenhäuser nicht vorgenommen wurde. Hierbei ist dem sachlogischen Zusammenhang zwischen den in den Rahmenentscheidungen festgelegten Zielen und den aus den Portfolios hergeleiteten Marketingstrategien Rechnung $z u$ tragen. In diesem Zusammenhang ist ebenso das Problem des Globalitätsgrades der zu entwickelnden Marketingstrategien zu diskutieren. Hierbei ist ein sinnvoller Ausgleich zwischen dem relativ hohen Aggregationsgrad der strategischen Geschäftseinheiten einerseits und den Anforderungen im Hinblick auf eine eindeutige Umsetzbarkeit der Basisstrategien in konkrete Marketinginstrumentalstrategien andererseits zu finden, damit operationale Handlungsanweisungen für nachgelagerte Planungsstufen formuliert werden können.

\section{$1.4 \mathrm{Zielsetzung} \mathrm{und} \mathrm{Gang} \mathrm{der} \mathrm{Untersuchung}$}

Die gegenwärtige Situation der Warenhäuser im Spannungsfeld der Betriebsformen macht eine langfristige, systematisch geplante Neuorientierung ihrer Marketingpolitik notwendig. Die Basis hierfür muß durch eine konsequente Anwendung der Denkmodelle der strategischen Marketingplanung gelegt werden. Zwar verfolgen Warenhäuser arteigene Ziele, dennoch ist zu erwarten, daß sich einige grundlegende 
Erkenntnisse der Portfolio-Planung zur Entwicklung von Marketingstrategien im Herstellerbereich auf ihre Marketingsituation und zielsetzung übertragen lassen. Dieses unter theoretischer und beispielhaft empirischer ${ }^{1}$ Orientierung aufzuzeigen, ist die zielsetzung der vorliegenden Arbeit.

Dazu müssen zunächst die notwendigen gedanklich-organisatorischen Voraussetzungen für den Einsatz der PortfolioMethode gelegt werden. Durch die Bildung strategischer Geschäftseinheiten als Planungsobjekte des Warenhauses erfolgt eine Strukturierung und Differenzierung in klar umrissene, isoliert $\mathrm{zu}$ planende Unternehmensbereiche von überschaubarem Komplexitätsgrad. Ihre Abgrenzung muß anhand von klaren warenhausspezifisch formulierten Kriterien erfolgen und kann nur teilweise den in Industrieunternehmungen gemachten Erfahrungen folgen.

Die Entwicklung von Marketingstrategien in Warenhäusern dient letzten Endes dazu, die übergeordneten Unternehmensund Marketingziele zu erreichen. Dementsprechend müssen die warenhausspezifischen Ziele im Einzelnen und in ihrem geordneten Zusammenhang im marktorientierten Zielsystem der Entwicklung von Marketingstrategien vorangestellt werden.

Weiterhin erfordert die detaillierte Entwicklung von Marketingstrategien zuvor eine eindeutige Positionierung des Warenhauses in seinem Markt. In diesem Zusammenhang sind einerseits die für das Warenhaus relevanten zielgruppen im Konsumentenmarkt aufzudecken und hinreichend

1 Die hierzu notwendige Informationsbasis entstammt z.T. aus Datenmaterial, das dem Verfasser von einem Warenhauskonzern zur Verfügung gestellt wurde. Um die Vertraulichkeit zu sichern, muß in einigen Fällen von einer Veröffentlichung der Datenherkunft abgesehen werden. 
zu beschreiben. Andererseits muß das generelle Verhalten gegenüber den unmittelbaren Warenhauskonkurrenten und den mittelbaren Wettbewerbern in den übrigen Betriebsformen des Handels so festgelegt werden, daß in den Augen der aktuellen und potentiellen Kunden ein eigenständiges Profil erreicht wird.

Im Mittelpunkt der Betrachtung steht die Entwicklung relevanter Marketingstrategien für Warenhäuser. Sie erfolgt auf der Basis der Portfolio-Methode und soll Aufschluß darüber geben

1. welche Portfolio-Varianten bei gegebenem Zielsystem und gegebener Positionierung der strategischen Marketungsituation von Warenhäusern adäquat sind und darauf aufbauend

2. welche strategischen StoBrichtungen (Marketing-BasisStrategien) sich aus der Position der strategischen Geschäftseinheiten ableiten lassen und nicht zuletzt, 3. welchen Beitrag die konsequente Anwendung der Portfolio-Methode zur Lösung relevanter Problemstellungen der strategischen Marketingplanung von Warenhäusern $\mathrm{zu}$ leisten vermag.

Die solchermaßen auf relativ hohem Globalitätsgrad entwickelten Basisstrategien müssen durch Marketing-Instrumentalstrategien konkretisiert werden. Es wird beispielhaft gezeigt, welche Ausprägungen die Marketing-Instrumentalstrategien für spezielle Basisstrategien aufweisen.

Die Durchsetzung und Kontrolle von Marketingstrategien in komplexen, arbeitsteiligen systemen wie Warenhäusern bedarf vielfältiger horizontaler und vertikaler Abstimmungsprozesse, die sich nur auf der Basis einer adäquaten $\mathrm{Ab}-$ lauf- und Aufbauorganisation vollziehen können. Infolgedessen zeigt das abschließende Kapitel einige zentrale Ansatzpunkte für die Integration und Koordination von Marketingstrategien sowie für die Realisierung eines marktorientierten strategischen Planungssystems in Warenhäusern. 
2 Abgrenzung und Bildung strategischer Geschäftseinheiten in Warenhäusern als Gegenstand der Portfolio-Planung

Die Komplexität von Warenhäusern, die $\in$ inerseits in der Breite und Tiefe des Sortiments und andererseits in der Zahl und Heterogenität der Filialen begründet liegt, macht eine Strukturierung im Sinne einer Gruppierung für die strategische Marketingplanung, insbesondere für den Einsatz der Portfolio-Methode (Festlegung des Planungsobjektes) notwendig. Dieser Problemkreis wird - bisher primär für Multiprodukt-Unternehmungen der Industrie - unter dem Stichwort "strategische Geschäftseinheiten" 1 diskutiert.

\subsection{Bedeutung der strategischen Geschäftseinheiten in Warenhäusern}

Das Konzept der strategischen Geschäftseinheiten ${ }^{2}$ ist zunächst in seiner allgemeinen Form darzustellen und kritisch $z u$ würdigen. Darauf aufbauend werden die Notwendigkeit des Denkens in strategischen Geschäftseinheiten für warenhäuser aufgezeigt, sowie konkrete - den Besonderheiten der Warenhäuser Rechnung tragende - Vorschläge zu ihrer Festlegung entwickelt.

1 Häufig werden Begriffe wie "Strategic Business Unit", "Geschäftsgebiet", "Geschäftsfeld", "Business" u.ä. synonym verwendet.

2 Vgl. zur Darstellung und Diskussion des Konzepts der strategischen Geschäftseinheiten im Überblick: Drumm, H.J., Planungs- und Anpassungsprobleme der Geschäftsbereichsorganisation, in: $\mathrm{Z} f \mathrm{~B} 48$ (1978), S. 87-104; Dunst, K., a.a.O., S. 56 ff.; Hinterhuber, H., Unternehmensplanung, a.a.O., S. 219 ff.; Derselbe, Die organisatorische Umsetzung der strategischen Planung in Unternehmen, in: $\mathrm{ZfB} 48$ (1978), S. 425-428; Roventa, P., a.a.O., S. 125 ff.; Stalp, H.-G., Strategische Geschäftseinheiten?, in: $\operatorname{ZfB} 48$ (1978), S. 919924 . 
2.11 Darstellung und Würdigung des Konzepts der strategischen Geschäftseinheiten

Als strategische Geschäftseinheiten werden üblicherweise Produkt/Markt-Kombinationen bezeichnet, die im Rahmen der strategischen Marketingplanung eine eigenständige Behandlung erfahren. Die strategischen Geschäftseinheiten sind so zu bilden, daß sie alle mit einem bestimmten "Geschäft" verbundenen strategischen Entscheidungen, Chancen und Risiken in ihrer Gesamtheit erkennen lassen.

Die Notwendigkeit der Bildung strategischer Geschäftseinheiten wird dann deutlich, wenn man als primäre Aufgabe der Unternehmensleitung die zieladäquate langfristige Steuerung der Unternehmung begreift. Die zunehmende interne und externe Komplexität des Systems und der stetige Wandel fordert eine "Zerlegung" des Gesamtsystems in überschaubare, im Rahmen klarer Strategien koordinierbarer Teileinheiten ${ }^{2}$ geradezu heraus. Hierdurch soll es gelingen, den Gesamtunternehmenserfolg durch die Erfolgssicherung auf disaggregierter Ebene - unter Beachtung der wechselseitigen Abhängigkeiten - sicherzustellen. Damit stehen Fragen der Bildung strategischer Geschäftseinheiten, die diesen Ansprüchen gerecht werden können, am Beginn jeglicher Überlegungen der strategischen Unternehmens- und Marketingplanung ${ }^{3}$.

1 Vgl. Gälweiler, A., Strategische Geschäftseinheiten (SGE) und Aufbau-Organisation der Unternehmung, in: ZO 5/1979, S. 252-260 (im folgenden zitiert als: Gälweiler, A., SGE); Henzler, H., Strategische Geschäftseinheiten (SGE) : Das Umsetzen von strategischer Planung in Organisation, in: $\mathrm{ZfB} 48$ (1978), S. 912-919 (im folgenden zitiert als: Henzler, H., SGE); Roventa, P., a.a.O., S. $125 \mathrm{ff.}$.

2 Hinterhuber spricht hier von einer "Aufteilung des Schlachtfeldes". Vgl. Hinterhuber,H., a.a.O., S. 221 f..

3 Selbstverständlich muß eine einmal durchgeführte $\mathrm{Ab}-$ grenzung während des strategischen Planungsprozesses stets überprüft und evtl. modifiziert werden. Vgl. hierzu die Ausführungen im Kapitel 1.22 der Arbeit. 
Angesichts der Bedeutung, die der Abgrenzung von strategischen Geschäftseinheiten zukommt, muß der bisher weitgehend uneinheitliche Stand der Literaturdiskussion erstaunen. Meist wird ein Katalog mit relativ alluemeinen Merkmalen aufgestellt, die als charakteristisch für eine strategische Geschäftseinheit gelten sollen oder es werden Anforderungen formuliert, denen eine strategische Geschäftseinheit entsprechen muß (vgl. hierzu die Beispiele in Tabelle 1 ). Hinzu kommt, daß die meisten Autoren Vorschläge zur Abgrenzung entwickeln, die auf Unternehmensbeispielen beruhen und von daher nur bedingt übertragbar sind ${ }^{1}$.

1 Als grundlegendes praktisches Beispiel gilt auch heute noch General Electric. Vgl. zur Beschreibung des General Electric-Konzepts z.B. Hall, W.K., SBU's: Hot Topic in the Management of Diversification, in: Business Horizons, Feb. 1978, S. 17-25; 0.V., Nichts geht durch die Lappen, in: MM 9/1979, S. 46-57; Springer, C.H., Strategi.c Management in General Electric, Operations Research, Nov.-Dec. 1973, S. 1177-1182. Weitere Vorschläge sind zu finden bei Albach, H., Unsicherheit, a.a.O., S. 712 f.; Ebskamp, K., a.a.O., S. $13 \mathrm{f}$.; Hanan, M., Reorganize your Company Around its Markets, in: Kotler, P., Cox, K. (Hrsg.), Marketing Management and Strategy. A Reader, Englewood Cliffs, N.J., 1980, S. 135-145; Hussey, D.E., a.a.O., S. 6; Hinterhuber, H., a.a.O., S. $214 \mathrm{f}$.; Lorange, P., Corporate Planning, Englewood Cliffs, N.J., 1980, S. 76 ff... Auch die PIMS-Studie basiert auf strategischen Geschäftseinheiten. Vgl. zu ihrer Bildung bzw. Definition: Abell, D.F., Hammond, J.S., a.a.O., S. 273; Neubauer, F.-F., a.a.O., S. 156 . 
A. Hinterhuber

1. Eigenständigkeit der Marktaufgabe

2. Gesellschaftsrelevanz der Marktaufgabe

3. Abhebung von der Konkurrenz

4. Erreichbarkeit relativer Wettbewerbsvorteile

5. Relative Unabhängigkeit der Entscheidungen

6. Führungseffizienz

B. Albach

1. Organisatorische Einheit

2. Eigenverantwortlichkeit durch selbständige Entscheidungen

3. Ausrichtung am Wettbewerber

C. General Electric

1. In sich geschlossenes homogenes Geschäft

2. Verantwortung des Managements für Strategien, Kontrolle und wichtige Funktionen

3. Ausrichtung an einem Hauptwettbewerber

D. $\underline{\mathrm{AEG}}$

1. Eindeutig abgrenzbare Produkt-bzw. Marktkombination

2. Einheitliche Zielbildungsmöglichkeit

3. Einheitliche Technologie

E. PIMS-Studie

1. Homogener Geschäftsbereich mit einer eigenständigen Marktaufgabe (kundenbezogen)

2. Klar abgrenzbares Produkt oder Produktgruppe

3. Klar bestimmarer Kreis von Wettbewerbern

Tab. 1: Abgrenzungskriterien und Anforderungen an strategische Geschäftseinheiten. 
Analysiert man die in der Tabelle 1 beispielhaft genannten Definitions- und Abgrenzungsvorschläge, so lassen sich unschwer folgende Mängel feststellen:

(1) Mangelnde Operationalität: die Abgrenzkriterien sind sehr allgemein gehalten und für eine konkrete Anwendung nicht exakt und handhabbar genug formuliert (Beispiel: Wie läßt sich die Gesellschaftsrelevanz, die Führungseffizienz oder die Unabhängigkeit nach Inhalt, Ausmaß und Zeitbezug präzisieren?).

(2) Konkurrenzbeziehung: eine Konkurrenz zwischen den unterschiedlichen Anforderungen wird eher die Regel als die Ausnahme bilden (Beispiel: Die Erreichbarkeit relativer Wettbewerbsvorteile impliziert die Schaffung möglichst großer und starker strategischer Geschäftseinheiten. Hier kann im Einzelfall ein Konflikt zur "Eigenständigkeit der Marktaufgabe" auftreten, die tendenziell klar abgegrenzte kleinere, an Produktoder Produktlinien orientierte Einheiten impliziert,

(3) Unbefriedigende Bereichsbildung: bei vorurteilsfreier Anwendung der Kriterien wird man häufig sich überlappende oder widersprechende Produkt/Markt-Kombinationen finden ${ }^{1}$.

Damit wird deutlich, daß solchermaßen festgelegte $A b-$ grenzungskriterien sowohl in theoretischer Hinsicht wegen ihrer mangelnden Präzision, Konsistenz oder Operationalität als auch für praktische Planungsaufgaben wegen der sich ergebenden problematischen,d.h. nicht eindeutigen und als objekt der strategischen Unternehmens- und Marketingplanung u.U. nicht unmittelbar einsetzbaren strategischen Geschäftseinheiten, unbefriedigend sind. Insofern darf es einerseits nicht verwundern, wenn in der Planungspraxis strategische Geschäftseinheiten gebil-

1 Vgl. Roventa, P., a.a.O., S. 126. 
det werden, die auf völlig anderen als den o.g. Kriterien beruhen ${ }^{1}$. Andererseits ist die mangelnde theoretische Fundierung des Konzepts sicherlich auch dafür verantwortlich, daß sich die Meinung eines Planungspraktikers in den Worten manifestiert: "In our company an SBU ultimately becomes watever subdivision corporate management wants it to be" ${ }^{2}$.

Vor dem Hintergrund dieser kritischen Beurteilung erscheint die unmodifizierte übertragung bisheriger Bemühungen zur Bildung strategischer Geschäftseinheiten für Warenhäuser nicht möglich. Vielmehr ist es notwendig, eine warenhausspezifische, realitätsnahe Analyse, Interpretation und Gewichtung der den bekannten Abgrenzungsvorschlägen gemeinsamen Abgrenzungs-Dimensionen "Produkt", "Markt", "Konkurrenz" und "interne struktur" vorzunehmen.

\subsection{2 Übertragbarkeit des Konzepts für Warenhäuser}

Die vielfältigen relevanten Unternehmens- und Umweltvariablen führen $z u$ einer Komplexität der Planungsaufgabe, die als Ganzes problematisch zu handhaben ist. Insofern erscheint es sinnvoll, eine Komplexitätsreduktion durch die Aufteilung der Gesamtunternehmung in solche Planungsobjekte bzw. Geschäftsbereiche vorzunehmen, die nach spezifischen externen und internen Aspekten gebildet sind und die Ableitung von operationalen Marketingstrategien ermöglichen. Gleichzeitig ist $\mathrm{zu}$ berücksichtigen, daß die für diese Teilbereiche abgeleiteten Marketingstrategien in ihrer Gesamtheit zuein-

1 Vgl. z.B. die Abgrenzung. von strategischen Geschäftseinheiten bei General Foods, beschrieben von Hall, W.K., a.a.O., S. 19 .

2 Ebenda. 
Die Dimension "Produkt" kann je nach vorgefundener Problemstellung unterschiedlich weit gefaßt werden. Darunter können Einzelprodukte, Produktgruppen jeweils nur mit ihrer Hard- bzw. Software oder auch mit ihrer Service- (Kundendienst-) Komponente verstanden werden. Als wichtig erscheint es, das Produkt funktionsorientiert zu betrachten, um eine langfristig stabile Definition zu erhalten ${ }^{1}$.

Die Dimension Produkt bzw. Ware erlangt insbesondere für Warenhäuser besondere Bedeutung, da die Zusammenstellung der Waren zu einem attraktiven Sortiment eine wesentliche Erfolgsdeterminante darstellt ${ }^{2}$. Dabei rückt hier insbesondere die Frage nach dem Aggregationsgrad der Dimension Ware in den Vordergrund. Gerade bei dem durch seine enorme Breite und Tiefe gekennzeichneten Warenhaussortiment ist das Problem der Bildung von höher aggregierten "Warenbündeln", die Gegenstand strategischer Entscheidungen sein können, evident.

Die Dimension "Markt" beinhaltet Aspekte der kunden- und regionalorientierten Segmentierung. Beide Aspekte sind bei Warenhäusern anders als bei üblicherweise analysierten Industrieunternehmen $\mathrm{zu}$ sehen.

Analysiert man die regionalen Besonderheiten der deutschen Warenhauskonzerne zunächst im Hinblick auf ihre länderspezifischen Strategien, so läßt sich eine fast ausschließliche Tätigkeit auf dem nationalen Markt feststellen ${ }^{3}$. Insofern bietet der üblicherweise bei Industrieunternehmen gewählte Ansatz, bei der Bildung strategischer Geschäftseinheiten verschiedene Länder zu berücksichtigen, kaum Ansatzpunkte ${ }^{4}$. Auch die isolier-

1 Vgl. Dunst, K., a.a.O., S. 57 f..

2 Dies gilt im Grunde für jede Einzelhandelsunternehmung.

3 Lediglich Karstadt unternimmt bislang erste Schritte durch eine Beteiligung an dem französischen Warenhauskonzern Jelmoli.

4 Allerdings mußte diese Beurteilung für den Fall verstärkter Internationalisierungsbemühungen geändert werden. 
te Analyse des inländischen Marktes zeigt kaum Ansatzpunkte für die Abgrenzung strategischer Geschäftseinheiten, da

- alle Konzerne überregional tätig sind, d.h., die gesamte Bundesrepublik abdecken

- die inländische Marktabdeckung insgesamt relativ gut ist, d.h., die Marktbesetzung mit zusammen fast $400^{1}$ Filialen relativ dicht ist und von daher

- insgesamt nur wenig unterversorgte Gebiete festzustellen sind; allenfalls konzernspezifisch ergeben sich einige Lücken ${ }^{2}$

- überwiegend national tätig sind und am Beginn ihrer Internationalisierung stehen.

Diese wenigen Statements zeigen auf, daß die geographische Betrachtung des Absatzmarktes im Augenblick noch keinen sinnvollen Ansatzpunkt zur Differenzierung bietet ${ }^{3}$. Berijcksichtigt man allerdings die zunehmenden Internationalisierungsbemühungen von Industrieunternehmen, so ist auch für Warenhäuser eine zukünftig stärkere Bedeutung des internationalen Geschäfts denkbar ${ }^{4}$. Zum gegenwärtigen Zeitpunkt scheint es jedoch gerechtfertigt $z u$ sein, die geographische Marktkomponente lediglich implizit zu berücksichtigen.

1 Vgl. hierzu die Tabelle 2 .

2 So ist der Tabelle 2 z.B. zu entnehmen, daß für HORTEN sich eine deutliche Unterbesetzung in den Ländern Schleswig-Holstein und Berlin ergibt; für den KAUFHOF ergibt sich die gleiche Aussage für die Länder Niedersachsen, Schleswig-Holstein und Berlin.

3 Diese Einschätzung trifft auch dann $z u$, wenn man z.B. eine Differenzierung des Marktes bzw. der Standorte, z.B. nach Ortsgrößenklassen durchführt.

$4 \mathrm{Vgl}$. zu den spezifischen Problemen hierbei z.B. Meffert, H., Marktsegmentierung und Standardisierung als absatzpolitische Probleme internationaler Unternehmungen, in: Zeitschrift für Wirtschafts- und Sozialwissenschaften, Heft 4 (1980) (in Vorbereitung);

derselbe, Marktsegmentierung und Marktwahl im internationalen Marketing, in: DBW, 37. Jg. (1977), S. 433-446. 


\begin{tabular}{|c|c|c|c|c|c|}
\hline & Horten & Kaufhof & Hertie & Karstadt & Gesamt \\
\hline $\begin{array}{l}\text { Nordrhein- } \\
\text { Westfalen }\end{array}$ & 21 & 38 & 19 & 60 & 138 \\
\hline Bayern & 7 & 11 & 7 & 14 & 39 \\
\hline Nieders. & 5 & 3 & 10 & 15 & 33 \\
\hline Hessen & 5 & 9 & 9 & 11 & 34 \\
\hline Rh.-Pfalz & 4 & 10 & 3 & 4 & 21 \\
\hline $\begin{array}{l}\text { Schleswig- } \\
\text { Holstein }\end{array}$ & 1 & - & 4 & 14 & 19 \\
\hline Bad. -württ. & 11 & 7 & 11 & 7 & 36 \\
\hline Berlin & 1 & - & 11 & 7 & 19 \\
\hline Bremen & 2 & 1 & 2 & 2 & 7 \\
\hline Hamburg & 3 & 2 & 5 & 13 & 23 \\
\hline Saarland & - & 3 & - & 1 & 4 \\
\hline Gesamt & $60^{+}$ & 84 & 81 & 148 & 373 \\
\hline \multicolumn{6}{|c|}{$\begin{array}{l}\text { + davon } 53 \text { Horten-Warenhäuser, } 6 \text { Merkur-Kaufhäuser, } \\
1 \text { Hanse-SB-Warenhaus und } 1 \text { City-Markt }\end{array}$} \\
\hline
\end{tabular}

Tab. 2 : Anzahl der Filialen der Warenhausunternehmen in den Bundesländern / Stand 31.12.1978 ${ }^{1}$

Analysiert man den zweiten Aspekt der Dimension "Markt", die Kundensegmentierung, so sind die Warenhäuser durch eine sehr breite zielgruppe gekennzeichnet. Versucht man, die Zielgruppe der Warenhäuser ${ }^{2}$ auf einer globalen Ebene anhand demographischer Abgrenzungsmerkmale $\mathrm{zu}$ beschreiben, so kann der typische Warenhauskunde 3 charakterisiert werden als:

- ca. 14 - 65 Jahre alt

- mit einem Haushaltsnettoeinkommen von mehr als $1000,-$ DM/Monat.

1 Quelle: ISB Waren- und Versandhäuser-Bericht 1979, Köln 1979, S. 147.

2 Die Kundenstruktur ist für alle vier Warenhauskonzerne nahezu identisch.

3 Die Angaben beruhen auf Marktforschungsergebnissen eines Warenhauskonzerns. 
Zieht man noch weitere demographische kriterien wie z.B. Beruf, geographische Verteilung, Ausbildung etc., heran, so zeigt sich, daB eigentlich von dem "typischen" Warenhauskunden gar nicht gesprochen werden kann: die demographische struktur der Warenhaliskunden entspricht weitgehend der durchschnittlichen Schichtung der Bevölkerung. Auch die Einbeziehung von psychographischen Kriterien läßt die operationale Abgrenzung auf der globalen, auf das gesamte Warenhaus bezogenen Segmentierungsebene kaum zu1. Damit rückt die Zielgruppe als isoliertes, eigenständiges Abgrenzungskriterium für strategische Geschäftseinheiten in Warenhäusern in den Hintergrund. Dies bedeutet allerdings nicht eine Vernachlässigung der kundenorientierten Aspekte, vielmehr gewinnen diese später im Zusammenhang mit den übrigen Dimensionen (insbesondere der Produktdimension) aus folgenden drei Gründen an Bedeutung:

(1) eine aussagefähige differenzierte Zielgruppenabgrenzung erscheint gegenwärtig nur auf einer niedrigeren Aggregationsebene, d.h. auf Warengruppen- (oder Artikelgruppen) ebene sinnvoll, wobei hierbei auch die standortbezogene Situation eine wesentliche Rolle spielt.

(2) Im Zuge einer stärkeren Profilierung der warenhäuser in der "Betriebsformenlandschaft" werden verstärkte Bemühungen um eine differenzierte Zielgruppenbearbeitung erkennbar2, so daß dieser Aspekt in Zukunft an Bedeutung zunehmen wird.

(3) Durch die unterschiedlichen Sortimentseigenschaften der Warengruppen sind gewisse Schwerpunktbildungen möglich.

$1 \mathrm{Vgl}$. hierzu die detaillierten Ausführungen in Kapitel 3.2 der Arbeit.

2 Diese Bemühungen finden ihren konkreten praktischen Niederschlag $\mathrm{u} . \mathrm{a}$. in der Schaffung von Boutiquen nach dem Shop in the Shop-Prinzip, durch eine bewußte Fachgeschäftsorientierung einzelner Warengruppen, oder durch die Betriebsformendiversifikation der Warenhäuser. Vgl. hierzu Krüger, H., a.a.0., S. 66 f.; Böckler, M., Ein unverwechselbares Profil aufbauen, in: W\&V Nr. 17, 27. April 1979, S. II-VI; Höller, P., a.a.O., S. 12 ff.; Alberts, K., Sortimentsfight statt Flächenklotzerei, in: ASW, 8/1979, S. 6-8; 0.V., Wachstum durch Lebensqualität, in: Wirtschaftswoche, Nr. 39, 22.9.1978, S. 16-24. Schenk, H.-O., Polarisierung die neue Handelsstrategie, in: Markenartikel, 10/1979, S. 578-589, hior S. 584 ff.. 
Die Dimension "Konkurrenz" erlangt für Industrieunternehmen besondere Bedeutung, da sich in ihr die orientierung der Unternehmensführung an externen Gegebenheiten manifestiert. Hinter diesem Aspekt steht die tuberlegung, daB als Voraussetzung zur zielerreichung Wettbewerbsvorteile aufgebaut, gehalten oder ausgenutzt werden müssen; was aufgrund der oligopolistischen Struktur auch für Warenhäuser gilt.

Allerdings kann bei Warenhäusern der Forderung nach der Ausrichtung an einem Hauptwettbewerber nicht gefolgt werden. Dies liegt in der Vielzahl der Konkurrenten begründet. Aufgrund der extensiven Branchenausrichtung und der breiten Marktabdeckung konkurrieren die Warenhäuser

- mit den übrigen Warenhauskonkurrenten (=unmitte1bare Konkurrenzebene)

- mit dem gesamten institutionellen Einzelhandel (mittelbare Konkurrenzebene).

Berücksichtigt man hierzu noch den grundsätzlichen regionalen, standortspezifischen Charakter des Wettbewerbs im Einzelhandel (außer Versandhandel), so wird deutlich, daß dieses Kriterium bei der Bildung von strategischen Geschäftseinheiten isoliert betrachtet keine herausragende Bedeutung aufweist. Damit rückt dieses Kriterium zwar bei der Abgrenzung der strategischen Geschäftseinheiten in den Hintergrund. Dies bedeutet allerdings nicht seine Vernachlässigung bei der Ableitung von zielen und Strategien, deren Formulierung selbstverständlich Konkurrenzaspekte vordringlich berücksichtigen muß.

Die Berücksichtigung der Dimension "interne Struktur" verhindert, daß bei der Abgrenzung von Planungsbereichen übersehen wird, daß Planung und reale interne Unternehmenssituation nicht isoliert voneinander betrachtet werden können. "Das heiBt, der zunächst nur begrifflich abgegrenzten SGE entspricht - spätestens im Ausführungsstadium stets ein konkretes Ausführungssystem" ${ }^{1}$. Damit wird der enge Zusammenhang zwischen der bestehenden Organisation und der Abgrenzung von strategischen Geschäftseinheiten deutlich.

1 Gälweiler, A., SGE, a.a.O., S. 253. 
Dies erscheint auch bei Warenhäusern von hervorragender Bedeutung, wobei insbesondere die filialmäßige Organisation berücksichtigt werden muB, da diese einige wichtige Konsequenzen im Hinblick auf

- die zentrale-dezentrale Planungskompetenz

- den zentralen-dezentralen Einsatz der Marketinginstrumente

- die Ressourcen usw. aufweist.

Faßt man diese isolierte Beurteilung der Dimensionen der strategischen Geschäftseinheiten zusammen, so rücken unter Berücksichtigung der Besonderheiten in Warenhäusern zunächst die Dimensionen Produkt (Ware) und interne Struktur (Filialorganisation) in den Vordergrund.

Somit lassen sich nunmehr als wichtigste, zum Gegenstand der strategischen Marketingplanung zu erhebende Teilbereiche ganz allgemein die "Ware" sowie die "Filialen" bezeichnen. Dies umso mehr, wenn man als primäre Aufgabe der Warenhausleitung das "Management von Waren" und das "Management von Filialen" interpretiert.

Diese bevorzugte Betrachtung zweier Dimensionen darf jedoch nicht zur völligen Vernachlässigung der übrigen Dimensionen bei der Abgrenzung von strategischen Geschäftseinheiten führen. Insofern erscheint eine heuristische Vorgehensweise sinnvoll, bei der die Bildung strategischer Geschäftseinheiten in Warenhäusern einmal aus isolierter Produktsicht (wobei die Dimensionen Markt, Konkurrenz und Filiale untergeordnet sind) und einmal aus isolierter Filialsicht (wobei die Dimensionen Markt, Konkurrenz und Produkt untergeordnet sind) erfolgt. Hierdurch gelingt es, das Problem zu strukturieren und vollständig zu durchdringen. 


\subsection{Bildung strategischer Geschäftseinheiten in Waren- häusern}

Entsprechend der bisherigen Diskussion werden die strategischen Geschäftseinheiten zunächst unter dem isoliertfilialbezogenen und dem isoliert-warenbezogenen Blickwinkel diskutiert. Dabei stehen insbesondere das Aggregations-Problem, die Berücksichtigung der übrigen $\mathrm{Ab}-$ grenzungsdimensionen sowie Probleme der Operationalisierung im Vordergrund.

\subsection{Strategische Geschäftseinheiten in Warenhäusern aus isolierter Filialsicht}

Für die herausragende Bedeutung der Filial(-organisation) bei strategischen Fragestellungen der Warenhäuser sprechen eine Reihe von Gründen. So gilt generell, daß jegliche Planungsaktivität sich grundsätzlich an den unternehmensinternen Faktoren (z.B. Personen, Sachmittel, Filialstruktur) orientieren muß. Als weiterer Grund ist die verstärkte aktuelle Diskussion in Theorie und Praxis bezüglich der Verteilung der Planungskompetenz, d.h. der Festlegung des Partizipationsgrades der dezentralen Stellen am Planungs- und Entscheidungsproze $\beta$ der überwiegend zentral geführten Warenhauskonzerne, zu nennen ${ }^{1}$.

Hierfür sprechen auch die verstärkten Bemühungen der Warenhauskonzerne im Rahmen des sogenannten "standort-

1 Vgl. hierzu z.B. Herder, H., Zentralisation, Teil II-IV, a.a.O.; Steffenhagen, H., Marktführer, a.a.O., S. $28 \mathrm{ff}$.; Derselbe, Zukunft fordert Strategie, in: SBWarenhaus 4/1979 (Teil I), S. 6-10 und 5/1979 (Teil II), S. 6-7 (im folgenden zitiert als: Steffenhagen, H., Strategie Teil I und Teil II). 
spezifischen Marketing" ${ }^{1}$, den einzelnen Filialen stärkere Mitspracherechte einzuräumen. Die Bedeutung der Filialen wird insbesondere deutlich, wenn man sich vor Augen hält, daß die Filialen vor ort jene Elemente des Gesamtsystems sind, die in direktem Kontakt zum Kunden stehen und damit auch wesentlich für den Erfolg verantwortlich sind ${ }^{2}$.

Für die Abgrenzung von strategischen Geschäftseinheiten ist zunächst festzuhalten, daB die einzelnen Filialen bei einer ersten Betrachtung als eigenständige Einheit angesehen werden können. Allerdings muß diese niedrige Aggregationsstufe als für strategische Planungsprobleme ungeeignet bezeichnet werden: Bei einer isolierten Betrachtung jeder einzelnen warenhausfiliale als strategische Geschäftseinheit wäre eine relativ unübersichtIiche Darstellung gegeben. Dies bedeutet, daß die Ableitung von Marketingstrategien für jede einzelne Filiale aus der Gesamtsicht heraus nicht mehr überschaubar und koordinierbar wäre, da die Vielfalt der Filialen eine Vielfalt der Marketingstrategien mit sich brächte. Es liegt deshalb nahe, Filialen mit gleichen oder ähnlichen Strukturen und Standortbedingungen zusammenzufassen und die solchermaßen gebildeten Filial- oder Haustypen als höher aggregierte Einheiten zum Gegenstand der strategischen Marketingplanung $z u$ erheben. Hierdurch würde eine Reduzierung der zu betrachtenden strategischen Geschäftseinheiten auf eine überschaubare Anzahl von Filialtypen erfolgen, für die operationale Marketingstrategien ableitbar wären.

1 Vgl. u.B. Käckenhoff, U., Wieder Schwung im Laden, in: Manager Magazin, 11/1979, S. 188-195, hier S. 192; 0.V., Sortimentsfight, a.a.o., s. $6 \mathrm{ff}$..

2 In diesem Zusammenhang wird in der Praxis oft der Ausdruck "all business is local" getätigt. 
Dieser Gedanke, die strategischen Geschäftseinheiten als Filialgruppen $\mathrm{zu}$ interpretieren, wirft insbesondere zwei noch zu klärende - allerdings höchst interdependente Fragen auf. Zum einen das Verhältnis bzw. der Zusammenhang zu den übrigen Dimensionen (Kunde, Konkurrenz, Ware) und zum anderen die Frage der Vorgehensweise bei der Gruppenbildung.

Unter primär filialorientiertem Blickwinkel gewinnt insbesondere die Konkurrenzdimension bereits bei der Bildung von strategischen Geschäftseinheiten an Bedeutung. Entsprechend dem festgelegten regionalen Charakter der Konkurrenzbeziehungen muß für jede Filiale die unmittelbare Warenhaus- sowie die mittelbare übrige Betriebsformenkonkurrenz in quantitativer (z.B. Marktanteil) und qualitativer (z.B. Imagevorteile) Form erfaßt werden. Darauf aufbauend werden jene (Einzel-) Filialen zu einer strategischen Geschäftseinheit zusammengefaßt, die in Bezug auf die Konkurrenzsituation ähnliche Strukturen aufweisen. Dabei sind als MeBkriterien (Subkriterien) z.B. die Art und Anzahl der Konkurrenten, die eigene Stellung gemessen am Marktanteil, das Verhalten der Wettbewerber (aggressiv, defensiv ...) denkbar. Mit dieser Berücksichtigung der Konkurrenzdimension wird ein üblicherweise in der Literatur gefordertes Abgrenzungskriterium für strategische Geschäftseinheiten berücksichtigt.

Die Betrachtung einzelner Filialen ermöglicht die Berücksichtigung speziell anzutreffender Zielgruppen bzw. Käuferstrukturen an jedem Standort. Somit läßt sich bei der Gruppierung zu Filialgruppen als Kriterium die Ähnlichkeit der Zielgruppen bereits als Gruppenbildungs-Kriterium mit einbeziehen. Dabei sind als MeBkriterien sowohl soziodemographische Kriterien (z.B. Kaufkraft, soziale Schichtung, Altersverteilung) als auch psychographische Kriterien (Einkaufsverhalten, Einkaufsstättenpräferenzen usw.) denkbar. 
Als Ergebnis der Gruppierung würden bei theoretisch exakter Vorgehensweise strategische Geschäftseinheiten vorliegen, die unter Zielgruppenaspekten identisch wären und damit die zielgruppenadäquate Ableitung von Marketingstrategien ermöglichten.

Die Berücksichtigung der Produkt- bzw. Warendimension kann durch die Art und Anzahl der geführten Artikel, durch die Sortimentsstruktur oder z.B. durch besondere Sortimentsschwerpunkte berücksichtigt werden. Die Gruppierung müBte somit nach dem Grad der Übereinstimmung in diesen sortimentsbeschreibenden Kriterien erfolgen.

In dieser Form gebildete strategische Geschäftseinheiten bieten ferner die Möglichkeit der Berücksichtigung weiterer, der 'internen Struktur' zuzurechnenden Kriterien. Bei der Gruppierung der Einzelfilialen können zusätzliche Kriterien herangezogen werden, die eine einzelne Filiale im Hinblick auf interne Faktoren (z.B. Größe, Standplatz usw.) beschreiben.

Zusammenfassend lassen sich als Trennkriterien, die die Unterschiede und Gemeinsamkeiten der einzelnen Filialen sichtbar machen, folgende Hauptkriterien nennen, die wiederum eine Reihe von Subkriterien unter sich vereinen können:

(1) Käuferschaft (z.B. Kaufkraft, soziale Schichtung, psychographische Kriterien)

(2) interne Struktur (z.B. Filialgröße, Verkehrslage,Standplatz)

(3) Sortiment (z.B. Struktur, Umfang, Schwerpunkte)

(4) Konkurrenz (z.B. Intensität, Verhaltensweisen)

(5) Sonstige Situationsfaktoren (z.B. Stadtstruktur, kulturelle Einflüsse).

Liegt die Beurteilung sämtlicher Häuser mit Hilfe dieser Kriterien vor, so kann eine Typologie erstellt werden,d.h. Häuser werden anhand von Ähnlichkeitsüberprüfungen zu mög- 
lichst homogenen Segmenten, Gruppen, Typen zusammengefaßt, die dann als strategische Geschäftseinheiten angesehen werden können. Dabei bietet sich zur Bildung von Filialgruppen eine Reihe von Planungsmethoden an. Als eine einfache heuristische Vorgehensweise bietet sich eine sukzessive Vorgehensweise an, die auf einer groben, subjektiven Ähnlichkeitsbeurteilung und Gruppenbildung anhand weniger Kriterien beruht. Die Abbildung 3 zeigt den Gedankengang an einem einfachen hypothetischen Beispiel mit 12 Filialen. Diese Vorgehensweise ist zweifellos nur eine recht grobe Heuristik, die insofern kritisch betrachtet werden muß, als

- nur ein Teil der relevanten Beurteilungskriterien und im realen Planungsfall zu berücksichtigenden Filialen einbezogen sind

- die Gruppenzuweisung aufgrund der Ähnlichkeit in den Kriterienausprägungen relativ vage ist und die (subjektive) Definition von Zuweisungs- bzw. Ablehnungsgrenzen notwendig macht,

- die Überschneidungen bzw. Anzahl der Filialen, die nicht eindeutig zuzuordnen sind mit steigenden Ähnlichkeitsansprüchen zunehmen.

Diese Kritikpunkte schränken die unbedenkliche Anwendung dieser einfachen Gruppierungsmethoden zwar ein, können jedoch erste Anregungen für die praktische Umsetzung des Grundgedankens geben. Unter theoretischen Aspekten ist zweifellos exakteren, modernen statistischen Verfahren wie z.B. der Clusteranalyse der Vorzug zu geben ${ }^{1}$. Der Grundgedanke der Clusteranalyse besteht darin, eine vorliegende Stichprobe nach bestimmten Kriterien in homogene Subgruppen aufzuteilen. Die dabei gebildeten Gruppen sollen sich dadurch auszeichnen, daß die in ihnen enthaltenen Elemente hinsichtlich bestimmter Kriterien

1 Vgl. zur Beschreibung dieser Methode z.B. Bock, H.H., Automatische Klassifikation. Theoretische und praktische Methoden zur Gruppierung und Strukturierung von Daten (Cluster-Analyse), Göttingen 1974; Everitt, B., ClusterAnalysis, London 1974; Hartigan,J.A., Clustering Algorithms, New York 1975; Hüttner, M., a.a.O., S. 380-407; Schuchard-Ficher, C. et al., Multivariate Analysemethoden, Berlin usw. 1980, S. 105-150. 


\begin{tabular}{|c|c|c|c|c|c|c|c|}
\hline Fillale & $\left|\begin{array}{c}\text { Umsatz } \\
\text { (M1O DM) }\end{array}\right|$ & $\begin{array}{l}\text { Umsatz- } \\
\text { rendite } \\
\text { (in 8) }\end{array}$ & $\begin{array}{l}\text { Sortiments- } \\
\text { struktur } \\
\text { (Typen/ } \\
\text { Klassen) } \\
\end{array}$ & $\begin{array}{l}\text { Konkurrenz- } \\
\text { intensität }\end{array}$ & Standort & $\begin{array}{l}\text { Stadt- } \\
\text { Charakter }\end{array}$ & $\begin{array}{l}\text { beispielhafte } \\
\text { heuristische } \\
\text { Gruppenbildung }\end{array}$ \\
\hline 1. & 160 & 2,5 & 5 & hoch & City & Weltstadt & Gruppe 1: \\
\hline 2. & 50 & 3,0 & 3 & hoch & City & & Fillalen $1,3,6$ \\
\hline 3. & 140 & 2,5 & 5 & hoch & $\begin{array}{l}\text { City/Fuß- } \\
\text { gängerzone }\end{array}$ & Weltstadt & Gruppe 2: \\
\hline 4. & 60 & 3,5 & 3 & niedrig & & Mittelstadt & Filialen $2,4,8$ \\
\hline 5. & 40 & 1,6 & 2 & mittel & City & Mittelstadt & Gruppe 3: \\
\hline 6. & 170 & 2,5 & 5 & hoch & $\begin{array}{l}\text { City/FuB- } \\
\text { gängerzone }\end{array}$ & Großstadt & $\overline{\text { Filialen }} 5,7,9,11$ \\
\hline 7. & 60 & 1,5 & 3 & mittel & $\begin{array}{l}\text { City/FuB- } \\
\text { gängerzone }\end{array}$ & Mittelstadt & Filiale 10 , isoliert \\
\hline 8. & 60 & 3,5 & 3 & niedrig & City & Kleinstadt & Filiale $12 \int$ be \\
\hline 9. & 70 & 1,4 & 3 & mittel & $\begin{array}{l}\text { C1ty/FuB- } \\
\text { gängerzone }\end{array}$ & Großstadt & \\
\hline 10. & 30 & 3,5 & 2 & niedrig & Rand lage & Kleinstadt & \\
\hline 11. & 70 & 1,5 & 3 & hoch & City & Großstadt & \\
\hline 12. & 60 & 0,5 & 3 & hoch & Vorort & weltstadt & \\
\hline
\end{tabular}


eine große Homogenität aufweisen, während die cluster untereinander möglichst heterogen sein sollen.

Hierin zeigt sich bereits der Wert dieser Methode für die Bildung von strategischen Geschäftseinheiten in Warenhäusern. Interpretiert man die Filialen als Elemente der Stichproben, beschreibt sie anhand der fünf Hauptund Subkriterien, so bildet die Clusteranalyse Filialgruppen, die intern homogen und extern heterogen sind. Damit ist die Möglichkeit zur Ableitung von gruppenspezifischen Marketingstrategien gegeben.

Aufgrund der Eignung dieser Methode erscheint es sinnvoll, eine Vorgehensweise zur Bildung der strategischen Geschäftseinheiten zu wählen, die sich an die Clusteranalyse anlehnt. Somit wird im folgenden - unter Verzicht auf statistische Details - ein 5-stufiges Vorgehen skizziert.

Stufe 1: Präzisierung des Untersuchungsziels Ziel der Clusteranalyse ist es hier, jene Filialen eines Warenhauskonzerns, die sich - gemessen anhand mehrerer Kri terien - ähnlich sind, zu Gruppen zusammenzufassen.

Stufe 2: Auswahl der Elemente und Variablen Die Elemente bilden sämtliche Filialen eines spezifischen Warenhauskonzerns. Die Variablen werden durch jene Faktoren oder Kriterien gebildet, anhana deren die einzelnen Filialen zu beurteilen sind. Dabei ist die Gesamtheit der zur Verfügung stehenden Kriterien zu differenzieren in aktive und passive Variablen. Die aktiven Variablen sind jene Variablen, die als Gruppierungsvariablen dienen, d.h. die Clusterbildung erfolgt zunächst nur aufgrund dieser Variablen. Diese Variablen müssen metrisch sein, womit aus den 0.9 . Set von Variablen die überwiegende Anzahl von Subkriterien herangezogen werden kann (z.B. Kaufkraft, Filialgröße, Sortimentsumfang). Die passiven Variablen dienen dazu, nach der clusterbildung die gebildeten cluster noch weitgehender zu beschreiben, bzw. nehmen damit eine gewisse - an realen Problemstellungen orientierte - Überprüfung der Clusterbildung vor. Im vorliegenden An- 
wendungsfall könnten hier z.B. die Sortimentsstruktur der einzelnen Filialen oder kulturelle Einflüsse des Standortes herangezogen werden.

Stufe 3: Festlegung der Ähnlichkeitsfunktion Die Gruppierung der Filialen erfolgt auf der Basis der vorliegenden Beurteilungen nach Maßgabe der Ähnlichkeit dieser Beurteilungen. Hierzu ist aus technischen Gründen die Definition eines Ähnlichkeitsmaßes ${ }^{1}$ notwendig, das den Abstand der Beurteilung zweier Filialen mißt ${ }^{2}$.

Stufe 4: Festlegung der Clustertechnik

In dieser primär statisch relevanten Stufe muB die Rechentechnik (der Clusteralgorithmus) festgelegt werden $^{3}$. Hier interessiert insbesondere die Frage nach der vorzugebenden Anzahl der Cluster 4 . Im vorliegenden Fall erscheint zunächst die Vorgabe einer relativ hohen Clusterzahl sinnvoll (ca. 20-25), die dann bei späteren Rechendurchgängen sukzessive verringert werden kann, bis eine gerade noch vertretbare clusterhomogenität erreicht ist.

Stufe 5: Interpretation der Ergebnisse

Nach der Durchführung der Rechnung mittels des Computers ist im letzten Schritt die Interpretation der clusterbildung - hier im Hinblick auf Fragestellungen der strategischen Marketingplanung notwendig.

Speziell ist zu fragen, ob die solchermaßen gebildeten Filialgruppen als strategische Geschäftseinheiten interpretierbar sind, d.h. die Ableitung von "ClusterStrategien" möglich ist.

1 In der Literatur werden verschiedene Ähnlichkeitsmaße diskutiert. Auf dieses Problem wird hier nicht eingegangen, da es sich um eine rein statistische Fragestellung handelt.Vgl. hierzu den guten Überblick bei Kaiser, A., Die Identifikation von Marktsegmenten, Berlin 1978, S. $186 \mathrm{ff} .$.

2 Neben der Ähnlichkeit zwischen den einzelnen Filialen ist es auch möglich, die Ähnlichkeit bzw. Unähnlichkeit zwischen den jeweils gebildeten Filialgruppen zu überprüfen. Dies ist mit Hilfe der Diskriminanzanalyse möglich, in deren Mittelpunkt die Ermittlung einer Geraden steht, die am schärfsten zwischen zwei Gruppen trennt (diskriminiert). Das bedeutet, daß die Zuweisung einer Filiale zu einer bestimmten Gruppe überprüft werden kann. Vgl. als Überblick zur Diskriminanzanalyse Hüttner, M., Informationen für Marketing-Entscheidungen, München 1979, S. 291-307 sowie die dort angegebene Literatur.

$3 \mathrm{Vgl}$. z.B. den guten Überblick über alternative Algorithmen bei Hüttner, M., a.a.O., S. $387 \mathrm{ff} .$.

$4 \mathrm{Vgl.}$..B. den Überblick bei Kaiser, A., a.a.O., S. $216 \mathrm{ff}$. sowie die dort angegebene Literatur. 
Der Einsatz der Clusteranalyse zur Bildung von strategischen Geschäftseinheiten in Warenhäusern genügt sicherlich hohen theoretischen Ansprüchen, doch dürfen die damit verbundenen Probleme insbesondere für den praktischen Einsatz nicht übersehen werden. Sie erfordert einen erheblichen Aufwand der Informationsgewinnung und -verarbeitung und fordert darüber hinaus hohe Anforderungen (z.B. metrische Skalierung) an die verfügbaren Informationen. So müssen für alle Filialen Analysen im Hinblick auf die Haupt- und Subkriterien durchgeführt werden ${ }^{1}$. Insbesondere die Quantifizierung von standortbezogenen Größen wie Marktanteil, Marktpotential oder Marktwachstum dürfte einige Schwierigkeițen bereiten ${ }^{2}$.

Vor dem Hintergrund dieser Schwierigkeiten wird in der realen Planungssituation ein Kompromi $\beta$ zwischen einer theoretisch wünschenswerten und einer praktisch realisierbaren Vorgehensweise notwendig sein. Unabhängig von der konkreten Ausgestaltung stellen die vorgeschlagenen Vorgehensweisen Ansatzpunkte dar, die

- zu relativ geschlossenen, d.h. unter nachfrage- und konkurrenzbezogenen Aspekten relativ homogenen Einheiten führen,

- eine Reduktion der großen Filialzahl bewirken und damit die Voraussetzung zu ihrer - unter marketingstrategischen Gesichtspunkten - eigenständigen Handhabung schaffen,

- die notwendige Flexibilität offenlassen, da jederzeit eine Umstrukturierung innerhalb der gebildeten Gruppen erfolgen kann (z.B. durch Hinzunahme einer neueröffneten Filiale).

1 Es ist allgemein bekannt, daß alle Warenhauskonzerne für fast jeden Standort, der bereits besetzt ist (oder als potentieller Standort gilt) Standortanalysen durchführen, die die o.g. Kriterien weitgehend berücksichtigen. Insofern ist dieser Einwand unter realen Planungsgegebenheiten evtl. nicht von großer Bedeutung.

2 Die Marktforschungsabteilungen der Warenhauskonzerne sind dabei in der Regel auf Expertenschätzungen angewiesen. 
Legt man diese Filialgruppen als strategische Geschäftseinheiten zugrunde, so ist die Voraussetzung geschaffen, daß die Warenhausleitung als primäre Aufgabe die Realisierung eines ausgewogenen Portfolios von Filialgruppen ansehen kann. Inwieweit diese positive Beurteilung für die aus primär warenorientierter sicht gebildeten strategischen Geschäftseinheiten auch gilt, ist im folgenden zu prüfen.

\subsection{Strategische Geschäftseinheiten in Warenhäusern bei isolierter Warensicht}

Aufgrund der elementaren Bedeutung der "Ware" bzw. des Sortiments ${ }^{1}$ für den Erfolg eines Warenhauses liegt es nahe, diesen Bereich als strategische Geschäftseinheiten zu interpretieren. Dabei drängen sich zwei Fragestellungen auf: Erstens, das Problem des Aggregationsgrades und zweitens, der Zusammenhang zu den übrigen Dimensionen (Kunden, Konkurrenz, interne struktur).

Die Warenhäuser sind durch eine große Vielzahl von Einzelprodukten gekennzeichnet. Große Warenhäuser führen z.B. 160.000

1 Die Bedeutung eines attraktiven Sortiments kommt auch darin zum Ausdruck, daß in diesem Zusammenhang von "Kernfunktion", "Grundfunktion" oder "grundlegender Leistung" gesprochen wird. Vgl. hierzu die Ausführungen bei Hansen, U., Absatz- und Beschaffungsmarketing des Einzelhandels, Band 1, Göttingen 1976, S. $194 \mathrm{f}$. sowie die dort angegebene Literatur (im folgenden zitiert als: Hansen, U., Marketing 1). Vgl. auch das grundlegende Werk zur Sortimentspolitik im Handel von Gümbel, R., Die Sortimentspolitik in den Betrieben des Wareneinzelhandels, Köln Opladen 1963.

2 Im folgenden soll die handelsbetriebswirtschaftliche Terminologie Sorte, Artikel und Warengruppen verwendet werden. 
Artikel und mehr ${ }^{1}$. Es bedarf keiner Begründung, daß diese disaggregierte Ebene nicht Gegenstand der strategischen Marketingplanung sein kann. Vielmehr ist - wie bei der Filialsicht - eine sinnvolle Gruppenbildung vorzunehmen, d.h. die disaggregierten Einheiten sind so zu bündeln, daß ausreichend globale, klar abgegrenzte Einheiten entstehen.

Analysiert man das Problem der Gruppenbildung zunächst aus formaler sicht, so ist das Gesamtsortiment in möglichst detaillierte, zugleich aber auch möglichst trennscharfe Einheiten zu klassifizieren. Diesen Anforderungen entspricht die Unterscheidung in "Sorte", "Artikel", "Artikelgruppen", "Warengruppen" sowie "Warenbereiche" ${ }^{2}$. Sie ermöglicht sowohl für die theoretische Analyse als auch für die praktische Planungsarbeit eine ausreichend detaillierte Klassifizierung der Sortimentseinheiten auf unterschiedlichen Aggregationsebenen ${ }^{3}$. Damit ist unmittelbar die Frage nach dem $z u$ wählenden Aggregationsgrad für strategische Fragestellungen aufgeworfen.

Betrachtet man zunächst die beiden Extrempunkte der Alternativen, zum einen die "Sorten" bzw. "Artikel" als kleinste Sortimentseinheiten und zum anderen die "Warenbereiche" (Textilien, Hartwaren und Lebensmittel) als globalste Einheit, so fällt eine Beurteilung relativ leicht. Die Sorten bzw. Artikel-Ebene ist für strategische problemstellungen zu differenziert. Die Entscheidungen über diese Sorti-

1 Vgl. Jeske, J., a.a.O., S. 15.

2 Vgl. zu den Klassifikationsvorschlägen u.a. Falk, B.R., Wolf, J., Handelsbetriebslehre, 4. Aufl., München 1979, S. 77 f.; Hansen, U., Marketing 1, a.a.O., S. 199 ff.; Meffert, H., Marketing, a.a.O., s. $372 \mathrm{f}$..

3 Hierfür spricht auch die positive Erfahrung der Warenhäuser in der realen planungssituation. 
mentsebene sind eindeutig der operativen Planung zuzurechnen. Die Warenbereiche sind zu global gefaßt, um operationale und differenzierte Marketingstrategien ableiten $\mathrm{zu}$ können.

Damit bietet sich eine Abgrenzung der strategischen Geschäftseinheiten unter Produkt-, Kunden- und Filialaspekten auf einem mittleren Aggregationsgrad an. Dieser für die strategische Planung geeignete Aggregationsgrad stellt die Warengruppe dar. Entsprechend der grundsätzlichen Anforderungen einer flexiblen und dynamischen Planung ist im Einzelfall durchaus auch die Modifikation der Abgrenzung im Hinblick auf die disaggregiertere Ebene der Artikelgruppe denkbar (vgl. Abbildung 4 ).

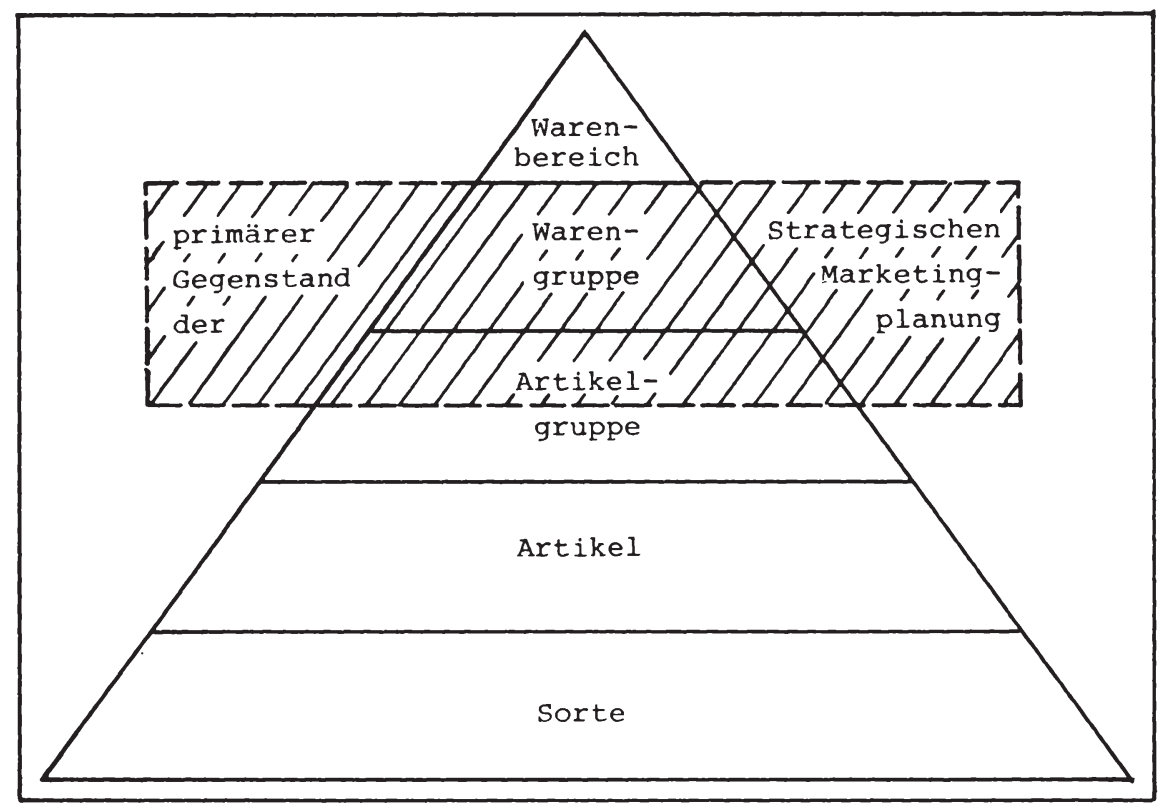

Abb. 4 : Sortimentshierarchie und strategische Marketingplanung 
Diese rein formale Betrachtung des Problems der Gruppenbildung reicht zur Bildung von strategischen Geschäftseinheiten in warenhäusern nicht aus. Vielmehr stellt sich nun die zentrale Frage zum einen nach der Bündelung der Sortimentseinheiten nach sog. Gestaltungsprinzipien ${ }^{1}$ und zum anderen nach der Berücksichtigung der drei übrigen Dimensionen (Kunde, Konkurrenz, interne Struktur).

Grundsätzlich sind als übergeordnete Gestaltungsprinzipien zu nennen:

- die Herkunftsorientierung, d.h. die Bildung des Sortiments aufgrund der Beschaffenheit des Materials (z.B. Textilien, Eisenwaren)

- die Preislagenorientierung, d.h. die starke preispolitische Ausrichtung der Sortimente (z.B. Billigpreis-, Hochpreissortimente)

- die Bedarfsorientierung, d.h. die Orientierung am Verbraucher bzw. an seinem Bedarf, unabhängig von der branchenmäßigen Zuordnung der Ware (z.B. Wohnen, Sport, Alles für das Kind)

- die Orientierung an der Selbstverkäuflichkeit der Ware, d.h. die Zusammenstellung des Sortiments nach Gesichtspunkten der Beratungs- bzw. Bedienungsnotwendigkeit der Wareñ.

$1 \mathrm{Vgl.} \mathrm{zur} \mathrm{ausführlichen} \mathrm{Beschreibung} \mathrm{der} \mathrm{Gestaltungs-}$ prinzipien Hansen, U., Marketing 1, a.a.0., S. 209 ff.; Nieschlag, R., Dichtl, E., Hörschgen, H., Marketing, 10. Aufl,, Berlin 1979, S. 212 ff.. 
Überprüft man die Bedeutung dieser Gestaltungsprinzipien für die strategische Marketingplanung in Warenhäusern bzw. für die Bildung strategischer Geschäftseinheiten, so ist gegen die Herkunftorientierung die primäre Ausrichtung an Produkteigenschaften, technischen Eigenschaften oder Beschaffungsaspekten vorzubringen, mithin die Vernachlässigung kundenspezifischer Gesichtspunkte. Gegen die isolierte Orientierung an der Preislage bzw. der Selbstverkäuflichkeit spricht die primäre Ausrichtung auf lediglich ein Marketinginstrument, was der Komplexität der strategischen Marketingentscheidungen in Warenhäusern nicht gerecht wird. Lediglich die Bedarfsorientierung sichert die Berücksichtigung der kundenspezifischen Belange und soll infolgedessen näher analysiert werden.

Die Bündelung der Sortimentseinheiten nach dem Bedarf der Konkurrenten kann sich an drei Prinzipien orientieren.

(1) Die Orientierung an Bedarfsarten besagt die relativ vollständige Abdeckung eines Bedarfsausschnittes für alle Konsumenten ${ }^{1}$. Aufgrund der unterschiedlichen Interpretationswerte der Bedarfsarten enthält das ausschließlich nach diesem Prinzip gebildete Sortiment "eine groBe Auswahl substitutiver Güter und führt zu einer wenig strukturierten Kundenselektion" ${ }^{2}$. Aus diesem Grunde ist dieses Prinzip in Warenhäusern in seiner reinen Form nur für wenige Bereiche des standardisierten Massenbedarfs (z.B. Haushaltswaren) anwendbar.

(2) Die Orientierung an Bedarfsträgern sieht die Sortimentsbildung aufgrund der spezifischen Ansprüche einer oder mehrerer Konsumentengruppen vor ${ }^{3}$. Damit wird die Basis für einen zielgruppenspezifischen Einsatz der Marketinginstrumente gelegt. Die insgesamt breite

$1 \mathrm{Vgl}$. zur Definition Hansen, U., Marketing 1, a.a.o., S. 210; Küthe spricht hier von der Orientierung an der "Problemlösung" (Vgl. Küthe, E., a.a.O., S. 95 f.).

2 Hansen, U., Marketing 1, a.a.O., S. 211.

$3 \mathrm{Vgl}$. ebenda. Vgl. Küthe, E., a.a.0., S. $95 \mathrm{f} .$. 
Zielgruppe der Warenhäuser sowie die oftmals nicht klar abgrenzbaren Bedarfsträger setzen einer isolierten Anwendung dieses Prinzips Grenzen.

(3) Eine dritte Möglichkeit stellt der Sortimentsaufbau nach Erlebnisbereichen dar. Der Bedarf wird dabei nach Erlebnisbereichen, die durch einen Komplex komplementärer Güter zu befriedigen sind, zusammengefaßt und bezieht sich meist auf bestimmte Aktivitäten der Kunden (z.B. "Schönsein", "zeitgemäßes Wohnen", "Do it yourself" usw.)" 1 . Dabei weist diese Art des Sortimentsaufbaus die Vorteile der Bedarfsorientierung und der Ausnutzung von Verbundkäufen auf. Insbesondere für Warenhäuser ergibt sich aufgrund der breiten Zielgruppe einerseits und der Vielfalt der Waren andererseits das Problem überschneidungsfreie (und damit kostengünstig handhabbare) attraktive Erlebniskomplexe zu erkennen und praktisch umzusetzen.

Diese knappen Ausführungen zeigen, daß die bedarfsorientierte Sortimentsbündelung in Warenhäusern keineswegs nur auf der Basis eines Gestaltungskriteriums erfolgen darf. Vielmehr muß das realisierte Warenhaussortiment das Ergebnis der kombinierten Anwendung der diskutierten Gestaltungsprinzipien sein. Diese Kombination kann ' dabei neben den bedarfsorientierten (Detail-) Prinzipien auch Aspekte der ubrigen Grundprinzipien beinhalten. Analysiert man die realisierte Sortimentsstruktur der deutschen Warenhäuser, so führen die Ubberlegungen zur Sortimentsbildung $\mathrm{zu}$ einer struktur, wie sie in der Tabelle 3 dargestellt ist.

1 Vgl. Hansen, U., Marketing 1, a.a.o., S. 211 ff.; Küthe, E., a.a.O., S. $97 \mathrm{ff..}$

2 Dabei können neben den 


\begin{tabular}{|c|c|c|}
\hline Warenbereich & Warengruppe & Artikelgruppe \\
\hline \multirow[t]{2}{*}{ Textilien } & $\begin{array}{l}\text { 1. Damen- } \\
\text { kleidung }\end{array}$ & $\begin{array}{ll}1.1 & \text { Röcke } \\
1.2 & \text { Hosen } \\
1.3 & \text { Hosenröcke } \\
1.4 & \text { Hüte } \\
1.5 & \text { Pelzbekleidung } \\
1.6 & \text { Lederbekleidung } \\
1.7 & \text { Blusen } \\
1.8 & \text { Kleider } \\
1.9 & \text { Kostüme } \\
1.10 & \text { Sportmoden } \\
1.11 & \text { Wollwaren } \\
1.12 & \text { Hauskleidung } \\
1.13 & \text { Berufskleidung } \\
1.14 & \text { Nachtwäsche } \\
1.15 & \text { Tageswäsche } \\
\end{array}$ \\
\hline & $\begin{array}{l}\text { 2. Herrenkleidung } \\
\text { 3. Alles fürs Kind } \\
\text { 4. Schuhe und } \\
\text { Kleintextilien } \\
\text { 5. Textiler Ein- } \\
\text { richtungsbe- } \\
\text { darf }\end{array}$ & $\vdots$ \\
\hline Hartwaren & $\begin{array}{l}\text { 6. Übriger Ein- } \\
\text { richtungsbedarf } \\
\text { 7. Einrichtungszu- } \\
\text { behör }\end{array}$ & \\
\hline Lebensmittel & $\begin{array}{l}\text { 9. Lebensmittel } \\
\text { 10. Restaurant/Café } \\
\text { 11. Sonstiges }\end{array}$ & \\
\hline
\end{tabular}

Tab. 3: Beispielhafte Abgrenzung von Sortimentsstrukturen 
Während die Ebene der Warenbereiche herkunftsorientiert strukturiert ist, stellt die Ebene der Warengruppen eine Vermischung von Bedarfsarten- und Bedarfsträgerprinzipien dar. So sind die Bereiche Damenkleidung, Herrenkleidung, Alles für's Kind primär bedarfsträgerorientiert zusammengefaßt, bei den übrigen Bereichen überwiegt die Bedarfsartenorientierung. Auf der Ebene der Artikelgruppe greift eine kaum noch nachvollziehbare Vermischung aller Gestaltungsprinzipien platz.

Die Interpretation der solchermaßen gekennzeichneten Sortimentsteile, insbesondere der Warengruppen als strategische Geschäftseinheiten in Warenhäusern bedarf noch einiger relativierender und kritischer Anmerkungen.

Analysiert man zunächst die Beziehungen zu den übrigen bei der Bildung strategischer Geschäftseinheiten in Warenhäusern zu diskutierenden Dimensionen, so sichert die primäre Bedarfsorientierung eine unmittelbare Berücksichtigung der Kundendimension ${ }^{1}$. Die Bündelung bzw. Aggregation des Bereichs Ware zu strategischen Geschäftseinheiten erfolgt unter expliziter Berücksichtigung der Kundendimension - ausgedrückt durch den Bedarf, der wiederum als Oberbegriff für Käuferverhalten, Käuferanforderungen, Kaufgewohnheiten usw. interpretiert werden kann. Die skizzierte Bildung warenorientierter strategischer Geschäftseinheiten ermöglicht weiterhin die Berücksichtigung der Dimension der internen Struktur. Dies betrifft z.B. die Beachtung von Beschaffungs- und Lagerungsaspekten, einer zielgruppengerechten Präsentation sowie der Filialorganisation. Betrachtet man die Filialorganisation näher,

1 Damit ähnelt die Vorgehensweise bei der Bildung strategischer Geschäftseinheiten aus warenorientierter Sicht sehr stark der für Industrieunternehmen typischen Bildung von Produkt-Markt-Segmenten. 
so ist an die Bündelung bzw. Aggregation der Waren die Forderung nach einer möglichst einfachen Umsetzung in der Filialorganisation $z u$ erheben. Diese Anforderung ist unter verkaufsstrategischen Gesichtspunkten zu sehen, da die Sortimentsstruktur und ihr Pendant in der Filialorganisation, die Abteilungsstruktur, stets im Zusammenhang gesehen werden müssen.

Die Konkurrenzdimension wird in der vorgeschlagenen Vorgehensweise zur Bildung warenorientierter strategischer Geschäftseinheiten nur nachrangig betrachtet. Ihre Berücksichtigung erscheint vielmehr erst bei der konkreten Ausgestaltung warengruppenbezogener Marketingstrategien relevant.

Darüber hinaus erscheint es insbesondere bei der primären Anwendung bedarfsorientierter Prinzipien wichtig, die strategischen Geschäftseinheiten dynamisch $z u$ interpretieren. Dies besagt, daB jedes Warenhaus den sich wandelnden Bedarfen, Einkaufsverhalten und Konkurrenzproblemen Rechnung tragen muß. Hierdurch bietet sich ein Innovationspotential für Warenhäuser, das bei rechtzeitigem Erkennen und Realisieren entscheidend zur Profilierung beitragen kann. Analysiert man unter diesem Aspekt die in der Tabelle 3 vorgestellte Sortimentsstruktur, so erscheint eine Modifizierung bzw. Verbesserung insbesondere im Hinblick auf die zunehmende Bedeutung des Freizeitbereichs für die Konsumenten wichtig. Hierzu müssen die unmittelbar die Freizeit berührenden Sortimentsbestandteile zu einer Warengruppe "Freizeit" zusammengefaßt werden, der z.B. die Artikelgruppen Sport, Reisen, Do it yourself usw. integriert. Ebenso vermag die Trennung textiler und sonstiger Einrichtungsgegenstände nicht einzuleuchten. Hier bietet sich die Zusammenfassung zu einer neuen Warengruppe "Wohnbedarf" an, die sämtliche Wohnprobleme (z.B. Möbel, Gardinen, Bettwäsche etc.) zusammenfaßt. 
Damit ist inhaltlich umrissen, welche Dimensionen bei der Abgrenzung der strategischen Geschäftseinheiten aus warenorientierter Sicht eine wesentliche Rolle spielen. Jede disaggregierte Sortimentseinheit ist anhand der folgenden Kriterien $\mathrm{zu}$ beurteilen und mit anderen, ähnlichen Elementen zu höher aggregierten Einheiten zusammenzufassen:

(1) Käuferschaft (z.B. Erlebnisbereich, Einkaufsverhalten, Produkterwartungen, Einstellungen)

(2) Filialorganisation (z.B. Abteilungsbildung, Verbundaspekte)

(3) Sonstige Situationsfaktoren (z.B. Beschaffungs- und Lieferverbund, notwendige Serviceleistungen).

Die theoretisch exakte Vorgehensweise zur Gruppenbildung erfolgt nun gleich wie bei der Bildung von Filialgruppen. Sämtliche Sortimentseinheiten werden anhand dieser Kriterien beurteilt und mittels geeigneter statistischer Verfahren zu Segmenten, Typen oder Gruppen unterschiedlichen Aggregationsgrades zusammengefaßt. Als statistisches Verfahren könnte auch hier wieder die Clusteranalyse angewendet werden. Gegen diese Vorgehensweise spricht die Vielzahl der $z u$ betrachtenden und $\mathrm{zu}$ beurteilenden Sortimentselemente auf der disaggregierten Ebene (z.B. Sorten oder Artikel). Der Aufwand für die Beschaffung aller relevanten Informationen ist entschieden $\mathrm{zu}$ hoch. Insofern ist für praktische Problemstellungen der strategischen Marketingplanung in Warenhäusern eine weniger exakte, primär von Intuition und Erfahrung geprägte Vorgehensweise üblich, die sich primär an der praktischen Umsetzbarkeit orientiert. Dabei wird eine Definition der Warenbereiche, Warengruppen und Artikelgruppen vorgenommen, die zwar unter dem Primat der Bedarfsorientierung steht, jedoch alle anderen Gestaltungsprinzipien ebenfalls umfaßt. Damit ergibt sich unter Bezugnahme auf die Beurteilungskriterien eine nicht nachvollziehbare Vermischung der drei Kriteriengruppen "Käuferschaft", "Filialprganisation" und "Sonstige Schlüsselfaktoren". 
Die Bildung von warengruppenbezogenen strategischen $\mathrm{Ge}-$ schäftseinheiten unter besonderer Berücksichtigung der bedarforientierten Aggregation bzw. Sortimentsstruktur sowie ihrer dynamischen Interpretation ist positiv zu beurteilen, da

- die primär unter käuferorientierten Gesichtspunkten aggregierten Warengruppen relativ homogene Einheiten darstellen

- ihr mittlerer Aggregationsgrad die Formulierung operativ umsetzbarer Strategien ermöglicht

- die Abgrenzung bzw. Bildung genügend Flexibilität für eine Umstrukturierung oder Ergänzung sowohl der Warengruppen als auch der Artikelgruppen innerhalb der einzelnen Warengruppen läßt

- die Berücksichtigung käuferspezifischer Aspekte bei der Aggregation einen Schritt auf dem Wege zur "marketingorientierten" Denkweise der Warenhäuser darstellt.

Allerdings ist hier relativierend $\mathrm{zu}$ bemerken, daß die beschriebene Vorgehensweise durchaus zur Bildung von Warengruppen im Sinne von strategischen Geschäftseinheiten führen kann, die in mehrfacher Hinsicht nicht mit dem bestehenden Sortiments- und den unmittelbar hieraus resultierenden Organisationsstrukturen der Warenhäuser übereinstimmt ${ }^{1}$. Die solchermaßen bestimmte Warengruppenstruktur wird nicht mehr identisch sein

- mit der einkaufsorientierten Warengruppenstruktur und führt damit evtl. zur Verschärfung des traditionellen "Konfliktes" zwischen (Zentral-) Einkauf und Verkauf in Warenhäusern sowie

1 Dies wird bei einer konsequent käuferorientierten Bündelung der Warengruppen sogar die Regel sein. Vgl. hierzu die beiden Vorschläge "Freizeit" und "Modernes Wohnen" auf Seite 65 der Arbeit. 
- mit der gegebenen Abteilungsstruktur und wird hier evtl. wesentliche organisatorische Anpassungen bedingen.

Ungeachtet dieser Kritikpunkte stellt die warenorientierte Bildung strategischer Geschäftseinheiten auf der Basis käuferspezifischer Bündelungskriterien einen vielversprechenden Ansatz dar, dieses Problem zu lösen und die Grundlage für die Ableitung von Marketingstrategien zu schaffen. Dies umso mehr, als die angeführten Kritikpunkte nur bedingt platz greifen, da die Bildung strategischer Geschäftseinheiten primär ein planerisches Problem und Fragen der organisatorischen Umsetzung erst nachgelagert zu diskutieren sind.

Damit sind zwei - jeweils isoliert betrachtet - durchaus sinnvolle Varianten der Bildung strategischer Geschäftseinheiten in Warenhäusern entwickelt, was die Frage nach ihrer vergleichenden Beurteilung aufwirft.

\subsection{Vergleichende Beurteilung der isolierten Ansätze}

An die zum einen aus Filialsicht und zum anderen aus Warensicht gebildeten strategischen Geschäftseinheiten der Warenhäuser sind eine Reihe von materiellen und formalen Anforderungskriterien oder Bedingungen zu richten. Die Erfüllung oder Nichterfüllung der Anforderungen sollte An$l a \beta$ zu einer Überprüfung der gewählten Abgrenzung sein. Im einzelnen sind folgende Anforderungen/Bedingungen $z u$ überprüfen, die primär aus den obigen definitorischen überlegungen zu strategischen Geschäftseinheiten sowie ihrer 
Eignung, Gegenstand der strategischen Marketingplanung zu sein, resultieren:

(1) Ganzheit- oder Homogenität besagt, daß die strategische Geschäftseinheit im Hinblick auf käuferbezogene Abgrenzungskriterien ein homogenes Geschäft darstellen muß, damit es als Ganzes strategisch planbar ist

(2) Eigenständigkeit besagt, daß eine eigenständige Aufgabe gegeben sein muß, die entweder darauf abzielt, ein bestimmtes Bedürfnis zu befriedigen oder einen bestimmten Markt zu versorgen

(3) Mittelbindung besagt, da 3 alternative Entscheidungen Auswirkung auf die Bindung oder Freisetzung von Ressourcen (Mitteln) haben

(4) Erfaßbarkeit besagt, daß eine klare und eindeutige Definition, Abgrenzung und Messung der strategischen Geschäftseinheit möglich ist

(5) Datenerhebung besagt, daß die zur strategischen Planung notwendigen Größen (z.B. Marktanteile, Marktwachstumsraten, Kundenverhalten, Konkurrenzsituation) erhoben und möglichst quantifiziert werden können

(6) Flexibilität besagt, daß bei festgestellten Veränderungen (intern/extern) Änderungen in der Definition und Abgrenzung berücksichtigt werden können 
(7) Kontrollierbarkeit besagt, daß die strategischen Geschäftseinheiten auch Gegenstand strategischer Kontrolle sein müssen

(8) Organisationsvereinbarkeit besact, daß die strategischen Geschäftseinheiten nicht im Widerspruch zur realisierten (Aufbau-) Organisation stehen, die für die Realisierung bzw. Umsetzung der Strategien zuständig ist.

Beurteilt man die zwei Alternativen anhand dieser Kriterien und wählt eine einfache Beurteijungsskala mit den Extrempunkten "erfüllt" und "nicht erfüllt" sowie eine Einstufung als "bedingt erfüllt" für jene Kriterien, deren Erfüllung näher zu bezeichnende Probleme aufwirft, so läßt sich anhand plausibler überlegungen das Profil der Abbildung 5 aufstelien.

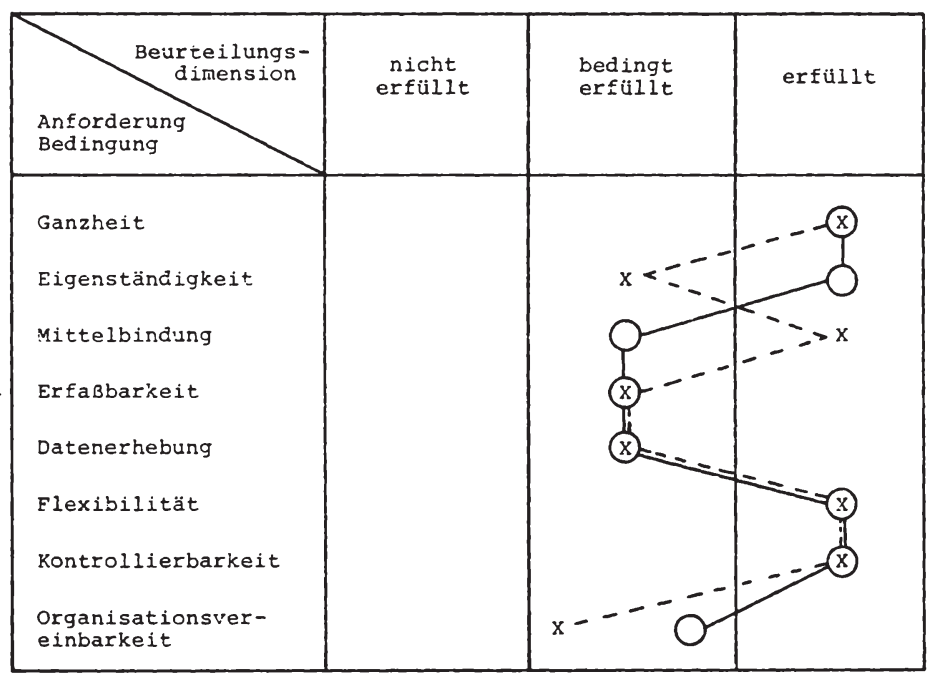

Legende :

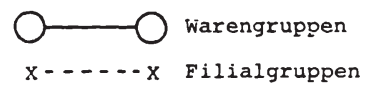

Abb. 5 : Beurteilung isoliert gebildeter strategischer Geschäftseinheiten 
Das Profil belegt auf den ersten Blick eine insgesamt relativ gute Erfüllung der Anforderungen sowie eine relativ starke Übereinstimmung der beiden Alternativen. Die Ganzheits-, Flexibilitäts- und Kontrollierbarkeitsbedingung sind eindeutig von beiden Alternativen erfült. Die Erfaßbarkeits- und Datenerhebungsbedingung ist bei beiden Alternativen nur bedingt erfüllt. Dies resultiert einerseits aus dem zweifellos großen Schwierigkeitsgrad, eine theoretisch weitgehend exakte Abgrenzung der Warengruppen und Filialgruppen zu realisieren und andererseits aus den Schwierigkeiten, alle für die Abgrenzung und die nachfolgende Ableitung von Marketingstrategien notwendigen Daten hinreichend genau bestimmen zu können. Diese negativ beurteilten Aspekte stellen jedoch nur Probleme der praktischen Umsetzbarkeit dar und können durch die Entwicklung und Anwendung geeigneter Meßverfahren in ihrer Bedeutung gemindert werden.

Die Erfüllung der Eigenständigkeitsbedingung ist tendenziell für Warengruppen besser erfüllt. Diese Beurteilung wird dann verständlich, wenn man sich die Bedeutung des Bedarfskriteriums bei der (theoretisch exakten) Bildung von Warengruppen vor Augen hält. Hier wird der Bedarf zum wichtigen Bildungsmerkmal erhoben, so daß seine Berücksichtigung ex definitione gesichert ist. Bei der Bildung von Filialgruppen steht zwar die Versorgung eines bestimmten "Marktes" im Vordergrund, doch ist die zugrundeliegende Definition des Marktes im Vergleich zur Bedarfsorientierung bei den Warengruppen nicht exakt genug. Bei der Bildung der Filialgruppen werden insbesondere kundenund marktorientierte (im Sinne von regional) Aspekte vermischt, d.h., es ist der Fall denkbar, daß Filialgruppen gebildet werden, die eine spezifische Zielgruppe in unterschiedlichen Regionen oder die in einer abgegrenzten Region unterschiedliche Zielgruppen bearbeiten. Dies muB für die Ableitung von Marketingstrategien kein Nachteil sein; bei strenger Auslegung der Eigenständigkeitsbedingung ergibt sich jedoch die eher negative Beurteilung. 
Bezüglich der Mittelbindungsbedingung wird die Filialgruppe besser eingeschätzt. Entscheidungen über Investitionen oder Desinvestitionen auf Filialgruppenebene (z.B. die Erweiterung der Filialgruppe um eine neue Filiale, oder die Schließung einer Filiale) haben starke Auswirkung auf die Bindung bzw. die Freisetzung von finanziellen Mitteln ${ }^{1}$. Dagegen sind die Auswirkungen bei Warengruppenentscheidungen (z.B. die Hinzunahme oder Aufgabe von Artikelgruppen in das Sortiment) bezüglich der einzusetzenden oder freiwerdenden Mittel in ihrer quantitativen Ausprägung tendenziell weniger bedeutsam ${ }^{2}$.

Bezüglich der Organisationsvereinbarkeitsbedingung werden beide Alternativen nur als bedingt geeignet angesehen, wobei die Warengruppenalternative leichte Vorteile aufweist. Hinter dieser einschränkenden Beurteilung steht die Uberlegung, daß eine konsequente strategische Planung nach dem Warengruppen- oder Filialgruppenprinzip Konflikte im Hinblick auf die realisierte Organisationsbzw. Planungsstruktur der Warenhäuser hervorrufen würde. Denn eine konsequente, durch strategische Geschäftseinheiten gekennzeichnete strategische Planung müßte hinsichtlich der Vèrteilung der Planungskompetenz von der gegenwärtig überwiegend zentralen zu einer eher dezentralen Planung übergehen. Die Warenhausleitung (oder die Zentrale) müßte sich konsequenterweise auf die Koordination und die Vorgabe des "Rahmens" beschränken, innerhalb dessen die strategischen Geschäftseinheiten autonom planen

1 Dies belegt auch ein Blick in die Warenhauspraxis, wo solche Entscheidungen finanzielle Auswirkungen von mehreren Millionen DM haben.

2 Hier wird aber auch die Interdependenz zwischen Warengruppen und Filialgruppen deutlich. So bedeutet die generelle Aufgabe einer Artikelgruppe auch die Freisetzung von Ressourcen in den einzelnen Filialen, da die bis dahin gebundenen Ressourcen (Verkaufsfläche, Personal, Finanzen) nun anderen Verwendungen zugeführt werden können.

3 Dabei ist $z$ u beachten, daß der Bedingung der Vereinbarkeit mit der Organisation bei dieser primär planerischen Fragestellung nur sekundäre Bedeutung zukommt. 
und entscheiden können. Es bedarf keiner weiteren Erläuterung, daß solche tiefgreifenden Änderungen in der Organisation und Führung der Warenhäuser sich nicht ad hoc, sondern vielmehr in einem langsamen evolutionären ProzeB vollziehen. Von daher kommt dem Denken in strategischen Geschäftseinheiten eine wesentliche Steuerungsfunktion bei der langfristigen Anpassung der Organisationsund Führungsstruktur zu.

Insgesamt dominiert zum einen keine Alternative eindeutig, und zum anderen erscheint eine konsequente planerische Durchführung unter Durchsetzungsgesichtspunkten problematisch zu sein. Diese beiden Aspekte legen die SchluBfolgerung nahe, eine Sichtweise zu wählen, die beiden Aspekten, d.h. der warenorientierten und der filialorientierten Sichtweise, Rechnung trägt und eine Planungssystematik zur Ableitung von Marketingstrategien zu entwickeln, die einen Kompromi $\beta$ aus primär zentraler und primär dezentraler strategischer planung darstellt.

\subsection{Duale Interpretation strategischer Geschäftseinheiten in Warenhäusern und ihre Implikationen für die stra- tegische Marketingplanung}

Die Beurteilung der beiden entwickelten Ansätze zur Bildung strategischer Geschäftseinheiten in Warenhäusern zeigt, daß die Filialgruppensicht und die Warengruppensicht im Grunde keine "echte" Alternativen darstellen. Vielmehr erscheint es aufgrund der Bedeutung beider Ansatzpunkte sinnvoll, eine duale strategische Marketingplanung durchzuführen, die zum einen die Planung von filialgruppenbezogenen Marketingstrategien und zum anderen die Planung von warengruppenbezogenen Strategien vorsieht. 
Aus der Perspektive der Gesamtunternehmung stehen bei der Entwicklung von Marketingstrategien für die Warengruppen und Filialgruppen vier grundsätzliche Strategietypen zur Verfügung (vgl. Abbildung 6 ). Berücksichtigt man, daß die reale Planungssituation der Warenhäuser von einem bestehenden Sortiment (als Summe aller Warengruppen) und einem bestehenden Filialsystem (als summe aller Filialgruppen) auszugehen hat, so muB im Mittelpunkt der Strategieableitung die Änderungsrichtung und -intensität der bisher verfolgten Strategien stehen. Somit bildet eine Expansionsstrategie den einen Extrempunkt des grundsätzlichen Entscheidungsspektrums der Warenhausleitung. Den Gegenpol hierzu bindet die Reduktionsstrategie, die durch eine Verringerung der Waren- bzw. Filialgruppen gekennzeichnet ist. Als "gemäßigte" Strategie zielt die Erhaltungsstrategie auf die Erhaltung des status quo ab. Die Konsolidierungsstrategie strebt einen vorsichtigen Ausbau von Waren- bzw. Filialgruppen an, wobei unter Renditegesichtspunkten schlecht beurteilte strategische Geschäftseinheiten eliminiert und neue nur dann geschaffen werden, wenn sich dadurch der Unternehmensertrag steigern läßt.

Aus der Kombination dieser beiden Dimensionen lassen sich die in der Abbildung 6 dargestellten, aufgrund von Plausibilitätsüberlegungen abgeleiteten globalen Marketingstrategien entwickeln. Sie reichen von Schrumpfungsüber Intensivierungs- und Konsolidierungsstrategien bis hin zu Diversifikationsstrategien. Dieses Strategieraster ${ }^{1}$

1 Dieses Strategieraster kann als modifizierte Ansoff'sche Produkt-Markt-Matrix interpretiert werden. Vgl. hierzu z.B. Ansoff, H.I., Management Strategie, a.a.O., S. 125 ff.; Derselbe, Strategies for Diversification, in: Taylor, B., Wills, W. (Hrsg.), Long-Range Planning for Marketing and Diversification, London 1971, S. 172-195. 
gibt zwar wertvolle Anregungen für gesamtunternehmensbezogene strategische Verhaltensweisen. Marketingstrategien lassen sich jedoch auf ihrer Basis wegen des $\mathrm{zu}$ hohen Aggregationsgrades kaum konkretisieren. Vielmehr

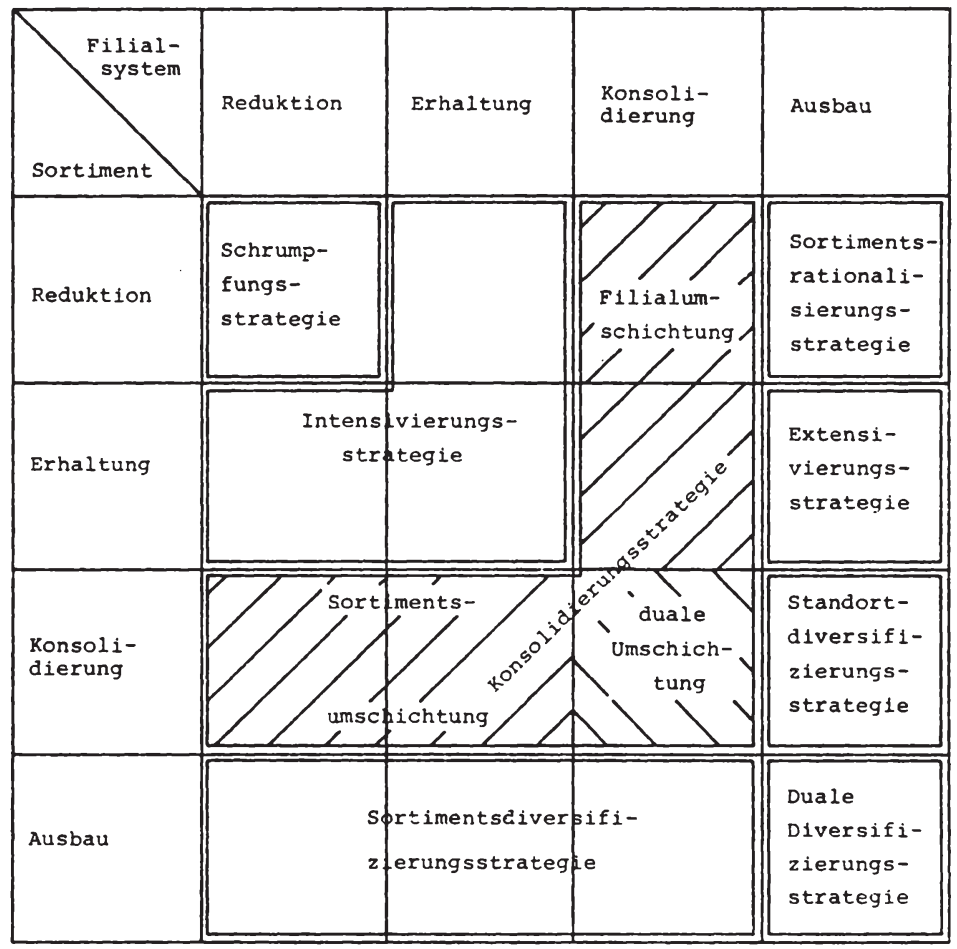

Abb. 6: Marketingstrategietypen auf der Basis einer Filialsystem-Sortiments-Matrix 
sind diese globalen Strategietypen im Rahmen des dualen Konzepts auf der Ebene der jeweiligen strategischen Geschäftseinheiten materiell auszufüllen.

Dabei führt die duale Planung aufgrund der Besonderheiten des jeweiligen Bereiches zu einem evtl. höheren Koordinationserfordernis, als dies bei der eindeutigen Dominanz einer Betrachtungsweise notwendig wäre. Neben geeigneten organisatorischen Konzepten ${ }^{1}$ (z.B. ständige Ausschüsse, teamorientierte Entscheidungen) kommt hier für die Fragestellungen der strategischen Marketingplanung vor allem den $\mathrm{Ziel-}$ und Positionierungsentscheidungen im Rahmen der strategischen Grobplanung besondere Bedeutung zu. Sie stecken den Rahmen für die nachfolgende strategische Feinplanung $a b$, engen damit die Freiheitsgrade der Entscheidung auf disaggregierter Ebene ein und bewirken somit eine Ausrichtung auf die Belange des Gesamtsystems.

Während auf der gesamtunternehmensbezogenen Ebene Warengruppen und Filialgruppen geplant werden, stehen auf der SGEEbene Artikelgruppen innerhalb einer Warengruppe bzw. einzelne Filialen innerhalb einer Filialgruppe zur Disposition. Insofern scheint es berechtigt, von einer "hierarchischdualistischen strategischen Marketingplanung" in Warenhäusern zu sprechen. Die Ableitung von Marketingstrategien bedarf daher sowohl in vertikaler als auch in horizontaler Hinsicht einer differenzierten Betrachtung, der beim Einsatz der Portfolio-Methode Rechnung zu tragen ist.

1 Diese sollen nicht näher erläutert werden, da hier Fragen der strategischen Planungssystematik im Vordergrund stehen. 
3. Ziele und Positionierung als Rahmenentscheidungen für die Portfolio-Planung in Warenhäusern

\subsection{Festlegung von Marketingzielen in Warenhäusern}

Die theoretische Erforschung der Ziele unternehmerischer Betätigung steht seit geraumer zeit im Vordergrund der modernen Betriebswirtschaftslehre. Auf besonderes Interesse stoßen dabei Fragestellungen zur Zielbildung, zur Projektion und zum Aufbau von Zielsystemen, wobei die Probleme primär aus der Herstellerperspektive diskutiert werden.

Aufgrund des besonderen situationalen Kontextes sowie der divergierenden Marketingaufgaben des Handels ergeben sich bei der Festlegung von Marketingzielen in Warenhäusern spezifische Probleme. Insofern werden im folgenden die relevanten Funktionen und Anforderungen an Ziele sowie Zielinhalte und Zielsysteme von Warenhäusern als Grundlage für die Portfolio-Planung diskutiert.

\subsection{Funktionen und Anforderungen marktorientierter ziele in Warenhäusern}

Marktorientierte Ziele des Warenhauses bilden die dem Marketingbereich gesetzten Imperative ${ }^{1}$ und verkörpern die erstrebenswerten Sollzustände ${ }^{2}$ des Warenhauses, die durch

1 Vgl. Heinen, E., Das Zielsystem der Unternehmung, 2. Aufl., Wiesbaden 1971, S. 51.

2 Vgl. Meffert, H., Unternehmensziele, in: Jahrbuch des Marketing, Ausgabe 1971, Hrsg.: Schöttle, K.M., Essen 1971, S. 22-34, hier S. 22, (im folgenden zitiert als: Meffert, H., Unternehmensziele). 
geeignete Marketingstrategien $\mathrm{zu}$ erreichen sind. Damit stellen sie die Endpunkte des strategischen Planungsfeldes in Warenhäusern dar, die den Rahmen für die inhaltliche und intensitätsmäßige Ausgestaltung der strategischen Handlungsweisen abstecken ${ }^{1}$.

Während die traditionelle Planungstheorie die Ziele von Unternehmen als gegeben voraussetzt, verläuft die zielbildung in Warenhäusern in der Realität als kreativer, multipersonaler Entscheidungs- bzw. Problemlösungsprozeß, der sich aus einer Vielzahl von Prozessen der Gewinnung, Verarbeitung und Weitergabe von Informationen sowie der interpersonellen Beeinflussung zusammensetzt ${ }^{2}$. An seinem Anfang stehen die gegenwärtigen Wert- und Zielvorstellungen des Warenhauses, die auf Willenskundgebungen des Managements bzw. des Vorstands basieren, sowie Erkenntnisse über Veränderungen der zukunftsbestimmenden Einflußgrößen im internen und externen Bereich des Warenhauses. Die Offenlegung und Prognose dieser Größen führt zu alternativen strategischen Zielvorstellungen, die hinsichtlich ihrer Konsequenz zu bewerten und vergleichen sind ${ }^{3}$.

1 Vgl. Zahn,E., a.a.0., S. 236.

2 Vgl. Bamberger, I., Grundprobleme und Forschungsansätze der langfristigen Zielplanung, in: ZO 2/1977, S. 91-99, hier S. 93. Vgl. auch Zahn, E., a.a.O., S. 238; Töpfer, A., a.a.O., S. 59 f.; Steiner, G.A., a.a.O., S. $140 \mathrm{ff.;}$ Pümpin, C., a.a.O., S. $24 \mathrm{ff.;} \mathrm{Wild,} \mathrm{J.,} \mathrm{Grundlagen,}$ a.a.O., S. 57 ff.; Heinen, E., a.a.O., S. 187 ff..

3 Hier können Größen z.B. aus dem Bereich der Konsumenten (Kaufkraft, Verhalten usw) der Konkurrenten (Betriebsformenentwicklung, Strategiewandel usw.) und der politischen Umwelt (Gesetzgebung, soziale Bestrebungen usw.) herangezogen werden.

Die Entwicklung solcher Zukunftsbilder basiert grundsätzlich auf der Anwendung von Prognosemethoden. Vgl. hierzu den guten Ủberblick bei Bamberger, I., a.a.O., S. 94 sowie die dort angegebene Literatur.

Zum Einsatz mathematischer Modelle bei diesem Problemkreis vgl. Meffert, H., Steffenhagen, H., MarketingPrognosemodelle, Stuttgart 1977. 
Dabei rücken im Rahmen der Bewertung Aspekte in den Vordergrund, die den politischen Charakter der Zielbilaung in Warenhäusern verdeutlichen. Im Rahmen der Konsensbildung werden die unterschiedlichen, von einzelnen Individuen oder Gruppen vorgebrachten ziele für das Warenhaus in Ziele des Warenhauses transformiert. Dazu müssen die vielfältigen Werte und ihre Veränderungen im Zeitablauf offengelegt und systematisiert werden.

Unter dem Blickwinkel der Durchsetzbarkeit sind die politisch gebildeten Zielvorstellungen im Zielsystem $z u$ ordnen und zu dokumentieren, um als verbindliche Richtlinien für untere Instanzen dienen zu können. Diesem Aspekt ist in Warenhäusern besondere Beachtung zu schenken, da aus dem zentral festgelegten Zielsystem unmittelbar die Steuerungsgrößen für die dezentralen Stellen, insbesondere die Filialen, abgeleitet werden müssen.

Da die ziele auf der vorgefundenen internen und externen Lagebeurteilung ${ }^{1}$ aufbauen, wirken sie als problemgeneratoren, d.h. sie setzen die Suche nach Strategien in Gang . Gleichzeitig übernehmen sie als Problemevaluatoren die Funktion von Entscheidungskriterien bei der Auswahl geeigneter Strategien? Durch die Vorgabe von zielen werden weiterhin die vielfältigen Aktivitäten im Warenhaus rechtzeitig koordiniert und die Kommunikation zwischen den dezentralen Entscheidungsstellen erleichtert. Kommt es zu Konfliktsituationen, so fungieren die $\mathrm{Ziele}$ als (rationale) Konflikthandhabungsmechanismen. Schließlich wird erst durch

1 Vgl. hierzu die (checklistenartigen) Vorschläge z.B. von Amara, R., Strategic Planning in a Changing Corporate Environment, in: LRP, Vol. 12, Febr. 1979, S. 2-16; Croon, P., Aids in Determing Strategy, the Internal Analysis, in: LRP, Vol. 12, Aug. 1979, S. 65-73; Hill, W., a.a.O., S. $289 \mathrm{ff.}$.

2 Vgl. auch Zahn, E., a.a.O., S. 250. 
die Zielvorgabe eine Kontrolle ${ }^{1}$ möglich, auf deren Basis die einzelnen im Warenhaus ablaufenden Prozesse gesteuert werden können ${ }^{2}$.

Diese vielfältigen Funktionen können die Marketingziele bzw. das zugrundegelegte Marketing-zielsystem nur dann erfüllen, wenn die vom zielsetzenden Gremium getroffenen zielentscheidungen bestimmten Anforderungen ${ }^{3}$ genügen.

Die strategischen Marketingziele müssen realistisch formuliert, d.h. an den jeweiligen Markt- und Betriebsgegebenheiten orientiert sein, da sie andernfalls als Utopien angesehen werden und ihre Motivationsfunktion verlieren.

Hierzu müssen die Ziele ebenfalls handlungsoperational sein. Sie müssen es dem jeweiligen Entscheidungs- und

1 Dabei ist es insbesondere für die strategische Marketingplanung wichtig, zielbeeinflussende interne und externe Veränderungen bereits rechtzeitig $\mathrm{zu}$ erkennen, um so in einem möglichst frühen Stadium eine Ziel- und Strategiekorrektur durchführen zu können. Eine Möglichkeit hierzu stellt die Anwendung des Leitlinienkonzeptes dar. Es basiert auf dem Grundgedanken, zu verschiedenen Zeitpunkten die Abweichungen von einer Planzielkurve zu messen, ihre Konsequenzen auf den tatsächlichen Zielkurvenverlauf zu prognostizieren und bei $\mathrm{Ab}-$ weichungen, die eine bestimmte Toleranzgrenze überschreiten, rechtzeitig Korrekturmaßnahmen zu ergreifen. Vgl. hierzu Crawford, C.M., Das Leitlinienkonzept in der Absatzplanung, in: Marketingtheorie, hrsg. v. Kroeber-Riel, W., Köln 1972, S. 254-269.

2 Vgl. zu den Funktionen z.B. Szyperski, N., Das Setzen von Zielen - Primäre Aufgabe der Unternehmensleitung, in: ZfB 41 (1971), S. 639-670; hier S. 651 (im folgenden zitiert als: Szyperski, N., Ziele); Meffert spricht hier von der Koordinierungs-, Kontroll- sowie Anreiz- und Befriedigungsfunktion; vgl. Meffert, H., Unternehmensziele, a.a.O., S. $23 \mathrm{f..}$

3 In der Literatur werden verschiedene Anforderungskataloge an ziele (allgemein) entwickelt, die hier auf Warenhäuser übertragen werden. Vgl. u.a. Bircher, B., a.a. O., S. $98 \mathrm{ff.;}$ Meffert, H., Unternehmensziele, a.a.O., S. 24 f.; Steiner, G.A., a.a.O., S. 156 ff.. 
Ausführungsträger ermöglichen, die Zielvorstellung durch praktisches Handeln zu verwirklichen und die zielerreichung $z u$ kontrollieren ${ }^{1}$. Dies bedingt, daß der Zielerreichungsgrad in eindeutig verständlichen Größen ausgedrückt wird und meßbar ist. Eine wesentliche Voraussetzung hierfür ist die möglichst eindeutige Formulierung der Marketingziele, die vier Aspekte umfaßt. Die Präzisierung des zielinhalts verlangt die inhaltliche Umschreibung des zieles (z.B. Umsatz, Marktanteil, ROI). Die Präzisierung des zu realisierenden Zielausmaßes fordert eine möglichst quantitativ ausgedrückte Angabe der Höhe des Anspruchsatzes (z.B. $12 \%$ Rendite, $28 \%$ Marktanteil). Die Präzisierung der zeitlichen Dimension verlangt die Festlegung der Zeitspanne innerhalb derer die Ziele erreicht werden müssen. Schließlich muB in der Zielformulierung auf das relevante Marktsegment bzw. die Kundengruppe Bezug genommen werden.

Diese Dimensionen müssen für den Bereich der strategischen Marketingplanung von Warenhäusern insbesondere hinsichtlich der Operationalität von Zielen relativiert werden. Aufgrund der relativ langen zeitlichen Erstreckung der Planungsperiode und der damit verbundenen Unsicherheiten, lassen sich die erst langfristig greifenden ziele $\mathrm{zu}$ Anfang meist nur in groben Umrissen fassen und vage, d.h. wenig operational, formulieren. Sie werden deshalb in den nachfolgenden Planungsstufen in einem iterativen ProzeB entsprechend des jeweiligen Kenntnisstand zunehmend präzisiert und neu formuliert ${ }^{2}$. Unter diesem Blickwinkel

1 Vgl. hierzu Frese,E., Kontrolle und Unternehmensführung Entscheidungs- und organisationstheoretische Grundfragen, Wiesbaden 1968; derselbe, Ziele als Führungsinstrumente - Kritische Anmerkungen zum "Management by Objectives" -, in: ZO 5/1971, S. 227-238; Heinen, E., a.a.O., S. 117 .

2 Vgl. hierzu u.a. Bircher, B., a.a.O., S. $147 \mathrm{ff.;}$ Ulrich, H., Unternehmenspolitik, Bern und Stuttgart 1979, S. 104 f.; Zahn, E., a.a.O., S. $238 \mathrm{ff.}$. 
müssen die Ziele ebenso ein hohes Maß an Flexibilität aufweisen. Im Falle unvorhergesehener Ereignisse muß diese eine schnelle Zielanpassung sicherstellen. Der Bezug zur Umwelt findet auch seinen Niederschlag in der Forderung, die Bestimmung der relativen Position des Warenhauses im Konkurrenzumfeld zu ermöglichen. Insofern erscheint es bei der strategisch bedeutsamen harten Wettbewerbskonfrontation der Warenhäuser sinnvoll, die Ziele so zu formulieren, daß sie einen Vergleich mit Konkurrenzunternehmen erieichtern.

Die vielfältigen Funktionen von Zielen sowie die umfassenden Anforderungen verdeutlichen, daß nur eine geordnete Mehrheit von Zielen, d.h. ein Zielsystem $^{1}$, Gegenstand

1 Die theoretische und empirische Analyse von Zielsystemen bildet seit langem einen Schwerpunkt der Zielforschung. Vgl. hierzu beispielhaft die grundlegenden Arbeiten von: Heinen, E., Das Zielsystem der Unternehmung, a.a.O.; Bidlingmaier, J., Zielkonflikte und Zielkompromisse im unternehmerischen EntscheidungsprozeB, Wiesbaden 1968; Schmidt-Sudhoff, U., Unternehmensziele und unternehmerisches Zielsystem, Wiesbaden 1967; Berthel, J., Zielorientierte Unternehmenssteuerung, die Formulierung operationaler Zielsysteme, Stuttgart 1973. Eine spezielle Analyse aus marketingorientierter Sicht ist z.B. zu finden bei: Bidlingmaier, J., Die Festlegung der Marketingziele im Rahmen der Unternehmenspolitik, in: GfM-Mitteilungen zur Markt- und Absatzforschung, 16. Jg. (1970), S. 88; derselbe, a.a.O., S. $71 \mathrm{ff}$. sowie die dort angegebene Literatur. Die Erforschung der Zielsysteme des Einzelhandels wurde lange Zeit vergleichsweise stiefmütterlich behandelt. Erst neuere Untersuchungen widmen sich diesem Problemkreis. Vgl. z.B. Barth, K., Führung, a.a.O., S. $144 \mathrm{ff.;}$ Klein-Blenkers, F., Unternehmensziele im Facheinzelhandel, in: Mitteilungen des Instituts für Handelsforschung an der Universität zu Köln, hrsg. v. E. Sundhoff, 24. Jg. 1972, S. 69 ff.; Kunt, W., Die Zielkonzeption der mittelständischen Einzelhandelsunternehmen, in: Sonderhefte der Mitteilungen des Instituts für Handelsforschung an der Universität zu Köln, hrsg. v. E. Sundhoff, Nr. 24, Köln 1975. 
der Planung von Zielen in Warenhäusern sein kann. Als Zielsystem in Warenhäusern kann grundsätzlich eine geordnete Menge von Zielvorstellungen (Zielbündel oder Zielmenge) bezeichnet werden, in der die langfristigen Zielentscheidungen der Unternehmung zum Ausdruck kommen. Zur Ableitung eines solchen Zielsystems müssen zunächst die relevanten, warenhausspezifischen ziele definiert werden.

\subsection{Relevante Ziele für die strategische Marketingplanung} in Warenhäusern

Die Notwendigkeit einer marktorientierten Unternehmensführung im Warenhaus bedingt, daß Zielinhalte, die üblicherweise als Unternehmensziele klassifiziert werden (z.B. Gewinn, ROI, Marktanteil, Umsatzwachstum) Gegenstand marketingorientierter Zielinhalte sind. Diese sind dementsprechend nach ihrer Bezugsebene bzw. nach ihrem Differenzierungsgrad in allgemeine Marketingziele und Marketinginstrumente-ziele zu systematisieren.

Im Rahmen der allgemeinen Marketingziele kommt im ökonomischen Bereich dem Gewinnstreben bei Warenhäusern eine hervorragende Eedeutung zu. Die Gründe hierfür sind äußerst vielschichtig. Im markwirtschaftlichen system ist eine Unternehmung auf Dauer nur bei Erzielung eines zu definierenden Mindestgewinns überlebensfähig, da dem Gewinn die Funktion eines Lenkungsinstruments für knappe Ressourcen zukommt und er somit nicht nur die Verzinsung des eingesetzten Kapitals und die Substanzerhaltung abdeckt, sondern u.U. auch zur Finanzierung von Wachstumsinvestitionen beitragen muß ${ }^{1}$. Ohne auf die mit dem Begriff "Gewinn"

1 Vgl. Barth, K., Führung, a.a.O., s. 146 f.. 
verbundenen heterogenen Gedankeninhalte näher einzugehen ${ }^{1}$ ist für Warenhäuser der "Gewinn" inhaltlich insbesondere als langfristig definierter Mindestgewinn in Prozenten vom eingesetzten Kapital aufzufassen. Diese relative Formulierung des Gewinnziels führt zur Integration eines Rentabilitätsquotienten (ROI = Return on Investment) in das Zielsystem der Warenhäuser.

Als weiteres Ziel, das im Einzelhandel generell hohe Priorität genießt ${ }^{2}$, kennzeichnet das Umsatzstreben die $\mathrm{Ab}-$ sicht eines Warenhauses, seinen wertmäßigen Absatz zu erhöhen. Neben der absoluten Vorgabe (z.B. x Millionen DM in 1981) gewinnen hier für Warenhäuser insbesondere relationale Formulierungen in Form von Kennziffern an Bedeutung $^{3}$. Hier sind insbesondere die Zielinhalte Umsatz pro qm und Umsatz pro Beschäftigten $z u$ nennen. In ihnen kommen die zentralen Leistungsfaktoren eines Warenhauses, die Raumleistung und die Personalleistung - jeweils bezogen auf den Umsatz - zum Ausdruck.

Wird das Ziel der Handelsspanne bzw. des Warenrohertrags allgemein als wichtiges ziel des Einzelhandels gesehen, so stellen die Warenhäuser in jüngster Zeit mehr auf den Deckungsbeitrag als Zielgröße ab. Dabei wird dieser üblicherweise definiert als Warenrohertrag minus Personalkosten ${ }^{4}$ und für Warengruppen bzw. Filialen oder Abtei-

1 Vgl. zur Begriffsexplikation z.B. Heinen, H., a.a.O., S. $59 \mathrm{ff.}$. Vergleiche auch den Uberblick bei Kunt, W., a.a.O., S. $57 \mathrm{ff}$. sowie die dort angegebene Literatur.

2 Vgl. hierzu die empirischen Untersuchungen von KleinBlenkers, F., a.a.O., S. 81 ff.; sowie Kunt, W., a.a.O., S. $96 \mathrm{ff}$..

3 Diese können auch als Kennziffern der "Produktivität" interpretiert werden.

4 Obgleich diese Definitionen im Vergleich zur differenzierten Deckungsbeitragsrechnung in Industrieunternehmungen unbefriedigend ist, spielt sie in der iPraxis der Warenhausplanung unter dem Gesichtspunkt einer ertragsorientierten Steuerung eine große Rolle. Immerhin weist sie gegenüber der Steuerung mittels des Warenrohertrages den Vorteil auf, die Personalkosten (die ca. $20 \%$ vom Umsatz betragen) verursachungsgerecht zuzurechnen. 
lungen differenziert ermittelt. Die daraus abgeleiteten Kennziffern Deckungsbeitrag pro qm bzw. Deckungsbeitrag pro Filiale oder Abteilung liegen der Sortimentssteuerung sowie der wirtschaftlichen Ausnutzung knapper Verkaufsflächen (Verkaufsflächenschlüssel) zugrunde ${ }^{1}$.

Neben diesen unternehmensintern orientierten Zlelinhalten stellt der Marktanteil im Hinblick auf den notwendigen Konkurrenzbezug in der strategischen Marketingplanung eine weitere wichtige Zielgröße dar. Er wird - ausgehend von der hier zugrundegelegten Konkurrenzdefinition ${ }^{2}$ - global gemessen

(1) als das Verhältnis des eigenen Umsatzes zum Umsatz des gesamten institutionellen Einzelhandels und

(2) als das Verhältnis des eigenen Umsatzes zum Umsatz aller Warenhäuser.

Im Zuge einer weiteren Differenzierung sind warengruppenspezifische Marktanteile (Umsatz der Warengruppe i des Warenhauses $j$ im Verhältnis zum Umsatz der Warengruppe $i$ beim gesamten institutionellen Einzelhandel oder bei allen Warenhäusern) und regionale Marktanteile (Umsatz einer Filiale im Verhältnis zum Umsatz des gesamten institutionellen Einzelhandels am Standort bzw. im Einzugegebiet) zu ermitteln.

Insbesondere unter marketingorientierter Perspektive sind diese quantitativen ökonomischen Zielinhalte um psychographische ziele zu ergänzen. Hier ist als bedeutsamer zielinhalt zunächst das Image zu nennen. Hierunter ist das aus der Vorstellung und/oder der Wahrnehmung entstandene Gesamtbild zu verstehen, das sich die öffent-

1 Vgl. auch Barth, K., Unternehmersführung, a.a.O., S. 166. 2 Vgl. Kap. 3.31 dieser Arbeit. 
lichkeit (Kunden, Nichtkunden, sonstige Öffentlichkeit) von einem spezifischen Warenhaus(konzern) macht ${ }^{1}$. Da sich das Image eines Warenhauses überwiegend aus den persönlichen Erfahrungen des einzelnen Kunden an einem spezifischen Standort oder sogar in einer speziellen $\mathrm{Ab}-$ teilung dieser Filiale bildet, ist dieses Imageziel im Hinblick auf Filialen, Warengruppen und Abteilungen zu differenzieren ${ }^{2}$.

In engem Zusammenhang zum Image eines Warenhauses sind die Zielinhalte Bekanntheit und Kundenbindung zu sehen. Die Bekanntheit stellt die Voraussetzung zur Bildung von Images bzw. Einstellungen und gleichzeitig einen wesentlichen Indikator für die Erklärung bzw. Prognose des Kundenverhaltens $\mathrm{dar}^{3}$. Ebenso wie das Image ist die Be-

$1 \mathrm{Vgl}$. hierzu z.B. Henseler, R., Imagepolitik in Betrieben des mittelständischen Facheinzelhandels, Göttingen 1978, S. 8; Klein-Blenkers, F., Imagepolitik im mittelständischen Facheinzelhandel, in: Handelsforschung heute. Festschrift zum 50-jährigen Bestehen der Forschungsstelle für den Handel, Schriftenreihe der Forschungsstelle für den Handel, Dritte Folge, Nr. 7, Berlin 1979, S.125 -136 ; Kook, W., Einstellungen zur Universität. Operationalisierung und empirische Prüfung verhaltenswissenschaftlicher Hypothesen am Beispiel der Stadtbevölkerung von Münster, Frankfurt usw. 1980, S. 34 ; Meffert, H., Marketing, a.a.0., S. 75; Spiegel, B., Die struktur der Meinungsverteilung im sozialen Feld, Bern und Stuttgart 1961, S. 36; Trommsdorff, V., Die Messung von Produktimages für das Marketing. Grundlagen und operationalisierung, Köln 1975, S. 5 ff.; Winkler, A., Kritische Überlegungen zur Tragfähigkeit des Image-Begriffs, in: JAVF, 19. Jg. (1973), Nr. 2, S. 147-156, hier S. 149.

2 Dahinter steht die These, daß Prädispositionen in Bezug auf einen Warenhauskonzern (dokumentiert als Prädispositionen in Bezug auf eine Filiale am Standort des Kunden) sich als Ergebnis der Prädisposition in Bezug auf die Abteilungen dieses Hauses ergeben. Vgl. hierzu Heemeyer, M., Psychographische Marktanalyse im Einzelhandel. Verhaltenswissenschaftliche und meßtheoretische Verankerung einer operationalen Befragungs- und Auswertungskonzeption, Diss. Siegen 1980, S. 27.

3 Vgl. hierzu z.B. Steffenhagen, H., Wirkungen absatzpolitischer Instrumente, Theorie und Messung der Marktreaktion, stuttgart 1978, S. $94 \mathrm{ff}$. (im folgenden zitiert als: Steffenhagen, H., Wirkungen). 
kanntheit - üblicherweise als Prozentsatz der Bevölkerung ausgedrückt, dem der Geschäftsname geläufig ist - gesamtunternehmensbezogen (überregional) und filialbezogen (regional) zu definieren ${ }^{1}$. Von Kundenbindung (bzw. Einkaufsstättenbindung)wird gesprochen, wenn ein bewußter, d.h. nicht zufälliger, auf einem Entscheidungsproze $B$ zwischen mehreren Alternativen beruhender wiederholter Kauf in einem bestimmten Warenhaus vorliegt ${ }^{2}$. Die Kundenbindung soll bereits als Warenhauskunden gewonnene Käuferschichten halten, ihr Abwandern zu anderen Betriebsformen verhindern und sie stärker an das Warenhaus binden. Damit soll auch für zukünftige Kaufentscheidungen eine stabile Prädisposition zum Aufsuchen eines bestimmten Warenhauses erreicht werden. Diese Ausrichtung auf die bestehende Käuferschicht darf jedoch nicht zu einer Vernachlässigung neuer interessanter Käuferschichten führen. Diesem Aspekt trägt die Zielsetzung der Neukundengewinnung Rechnung. Es sollen neue Käuferschichten systematisch erschlossen werden, um eine Transformation von bisherigen Nichtwarenhauskunden $\mathrm{zu}$ Warenhauskunden $\mathrm{zu}$ vollziehen.

Zum Zwecke einer möglichst operationalen und umsetzungsorientierten Zielformulierung sind die allgemeinen Marketingziele (= Marketing-Oberziele) in Marketing-Instrumentalziele (= Marketing-Unterziele) zu transformieren. Da$\mathrm{zu}$ sind für die Bereiche des

- Leistungsmix (z.B. Sortimentsprofilierung, Eigenmarkenanteil, Sortimentsstruktur-Kennziffern)

- Kontrahierungsmix (z.B. Kalkulation, Preisniveausicherung)

1 Auch ein abteilungs- oder warengruppenspezifischer Bekanntheitsgrad ist $z$ u ermitteln. Vgl. hierzu die Ausführungen von Heemeyer, H., a.a.O., S. $34 \mathrm{ff.}$

2 Vgl. die ausführliche Begriffsdefinition und die Hinweise auf die Spezialliteratur bei Heemeyer, H., a.a.O., S. $268 \mathrm{ff}$, sowie bei Heinemann, M., Einkaufsstättenwahl und Firmentreue des Konsumenten, Diss. Münster 1974 , S. 12 ff.. 
- Distributionsmix (z.B. Rationalisierung, Marktabdeckungsgrad) und des

- Kommunikationsmix (z.B. Bekanntheitsgrad, Kontakthäufigkeit und -qualität, Verhältnis Selbst- zu Fremdbedienung)

spezifische Ziele zu konkretisieren und formulieren.

Die verschiedenartigen Marketingzielsetzungen der Warenhäuser sowie die vielfältigen Interdependenzen zwischen ihnen begründen die Notwendigkeit, die einzelnen ziele unter Beachtung ihrer komplementären, konkurrierenden oder indifferenten Beziehungen ${ }^{1}$ in ein Zielsystem zu integrieren.

1 Komplementäre Ziele liegen dann vor, wenn durch die Erfüllung eines Zieles auch die Erfüllung anderer Ziele gesteigert wird. Ziele konkurrieren miteinander, wenn die Erfüllung eines Zieles zur Minderung des Erfüllungsgrades der anderen ziele führt. Schließlich verhalten sich Ziele untereinander indifferent oder neutral, wenn die Erfüllung eines Zieles auf die Erfüllung der anderen Ziele keinen Einfluß ausübt. Vgl. zu dieser Begriffsabgrenzung Heinen, E., a.a.O., S. $94 \mathrm{ff}$.. Insbesondere bei Vorliegen konkurrierender Zielbeziehungen mu $\mathrm{die}$ Unternehmensleitung Methoden entwickeln, die einen KompromiB herbeiführen. Während für quantifizierbare Ziele analytische Methoden wie z.B. die lineare Programmierung oder die Vektor-Optimierung zur Konfliktlösung eingesetzt werden können (vgl. hierzu Laukamm, T., Zielkonflikte in der Unternehmensplanung, in: Harvard Manager, 3/1980, S. 92-97) sind für die Gesamtheit der Marketingziele, die auch psychographische Ziele einschließen i.d.R. andere Abstimmungsregeln anzuwenden. Ein häufig eingesetztes Verfahren stellt die Gewichtung von Zielen, d.h. die Entwicklung einer Prioritätenreihenfolge dar. Einer solchen Rangordnung kommt die Funktion $z u$, in Konfliktsituationen die Erfüllung eines spezifischen Zieles den Vorrang zu geben und damit gleichzeitig die Mindererfüllung eines (oder mehrerer) anderer Ziele in Kauf zu nehmen (vgl. hierzu z.B. Heinen, E., a.a.O., S. $107 \mathrm{ff.;}$ Meffert, H., Unternehmensziele, a.a.O., S. 30). Eine weitere, insbesondere dem praktischen Denken entgegenkommende ordnung der ziele stellt die Erstellung von Ziel-Mittel-Hierarchien (Instrumentalrelationen) dar, die im weiteren verfolgt werden. 
3.13 Bildung marketingorientierter Zielsysteme in Warenhäusern

Als Ausgangspunkt für die Ordnung der Marketingziele bieten sich im Hinblick auf das praktische Denken insbesondere die zwischen ihnen bestehenden Instrumentalbeziehungen an ${ }^{1}$. Sie begründen ein Rangverhältnis der ziele, ermöglichen die Einteilung in Ober- und Unterziele und damit die Bildung von Zielhierarchien. Die Grundlage für die Ordnung der ziele bilden entweder definitionslogische Beziehungen zwischen Zielen, aufgrund derer die oberziele in Partialziele (Teilkomponenten) zerlegt werden, die nur einen Teil der Zustandsbeschreibung des Oberziels umfassen, und/oder empirisch ermittelte Mittel-Zweck-Beziehungen, aufgrund derer die Unterziele als Mittel zur Realisierung angestrebter übergeordneter Zustände interpretiert werden können. Die Ordnung der Ziele nach dem Mittel-ZweckVerhältnis führt damit zu zielhierarchien, die ein zusammenfassendes Abbild aller Aufgaben der Organisationseinheiten ${ }^{2}$ darstellen. Die hierarchische Zielstruktur gibt Aufschluß über die operationale Umsetzung bzw. Ableitung von Marketingzielen für nachgelagerte Instanzen bzw. für taktische oder operative Problemstellungen ${ }^{3}$. Hierin ist ihre besondere Bedeutung für dezentral organisierte Unternehmungen wie Warenhäuser zu sehen, die damit die Probleme der Bildung von Bereichszielen lösen können und der zielorientierten Steuerung des Gesamtunternehmens näher kommen.

1 Vgl. hierzu z.B. Heinen, E., a.a.O., S. 102 ff.; Kupsch, P., a.a.O., S. 33 ff.; Meffert, H., Unternehmensziele, a.a.o., S. $30 \mathrm{f}$..

2 Vgl. hierzu Hauschildt, J., Zielhierarchien in innovativen Entscheidungsprozessen, in: Unternehmensplanung, Hrsg. Ulrich, H., Wiesbaden 1975, S. 103-132, hier S. 114.

3 Die Bedeutung dieses Aspektes hat z.B. Hauschildt empirisch nachgewiesen. Er belegt, daß Entscheidungsträger bei innovativen Problemen dazu neigen, auf ziele höher- und niedrigerer hierarchischer ordnung zurückzugreifen. Dies setzt natürlich das Vorhandensein eines solchen Systems voraus. Vgl. Hauschildt, J., a.a.O., S. 116 f.; Derselbe, Zielbildung - ein heuristischer Proze $B$, in: $\mathrm{ZfB} 76, \mathrm{~S} .328-340$. 
Die Struktur eines solchen hierarchischen Zielsystems für Warenhäuser zeigt beispielhaft ${ }^{1}$ die Abbildung 7 . Aus der grundlegenden Philosophie des Warenhauses leiten sich die Unternehmensziele (= Oberziele) wie z.B. der Gewinn bzw. ROI, Umsatzwachstum, Marktanteil, Kostenwirtschaftlichkeit und Sicherheit $\mathrm{ab}^{2}$. Aus den Oberzielen sind die Umsatzziele und der Marktanteil in die allgemeinen Marketingziele (= Marketing-Oberziele) zu übernehmen. Hinzu treten das Image, der Deckungsbeitrag, der Umsatz pro Beschäftigte, die Handelsspanne, der Warenumschlag, die Kundentreue, die Gewinnung neuer Käuferschichten usw. Diese Marketing-Oberziele finden ihre Konkretisierung in den Marketinginstrumente-Zielen des Warenhauses, die sich in die Unterzielbereiche der Produkt- und Sortiments-, der Kontrahierungs-, der Distributions- sowie der Kommunikationspolitik differenzieren lassen.

Ein solchermaßen grob skizziertes marketingorientiertes Zielsystem von Warenhäusern bedarf einer weiterführenden Analyse in zwei Richtungen. Zum einen ist den organisatorischen Besonderheiten der Warenhäuser durch die Ableitung spezifischer Bereichsziele Rechnung zu tragen, zum anderen sind die Interdependenzrelationen einer detaillierten Analyse zu unterziehen. Insbesondere aufgrund des hierarchisch-dualistischen Charakters der strategischen Mar-

1 Die Multidimensionalität der ziele, ihre i.d.R. nur unvollständig zu erklärenden Zielrelationen und vor allem die allenfalls unternehmenspezifischen Präferenzen hinsichtlich der Zielinhalte erlauben nur eine beispielhafte, auf Plausibilitätsüberlegungen und praktischen Erfahrungen beruhende Strukturierung eines marketingorientierten Zielsystems von Warenhäusern.

2 Innerhalb der Oberziele muß wiederum eine entscheidungsträgerabhängige Gewichtung (Prioritätenreihenfolge) durchgeführt werden. 


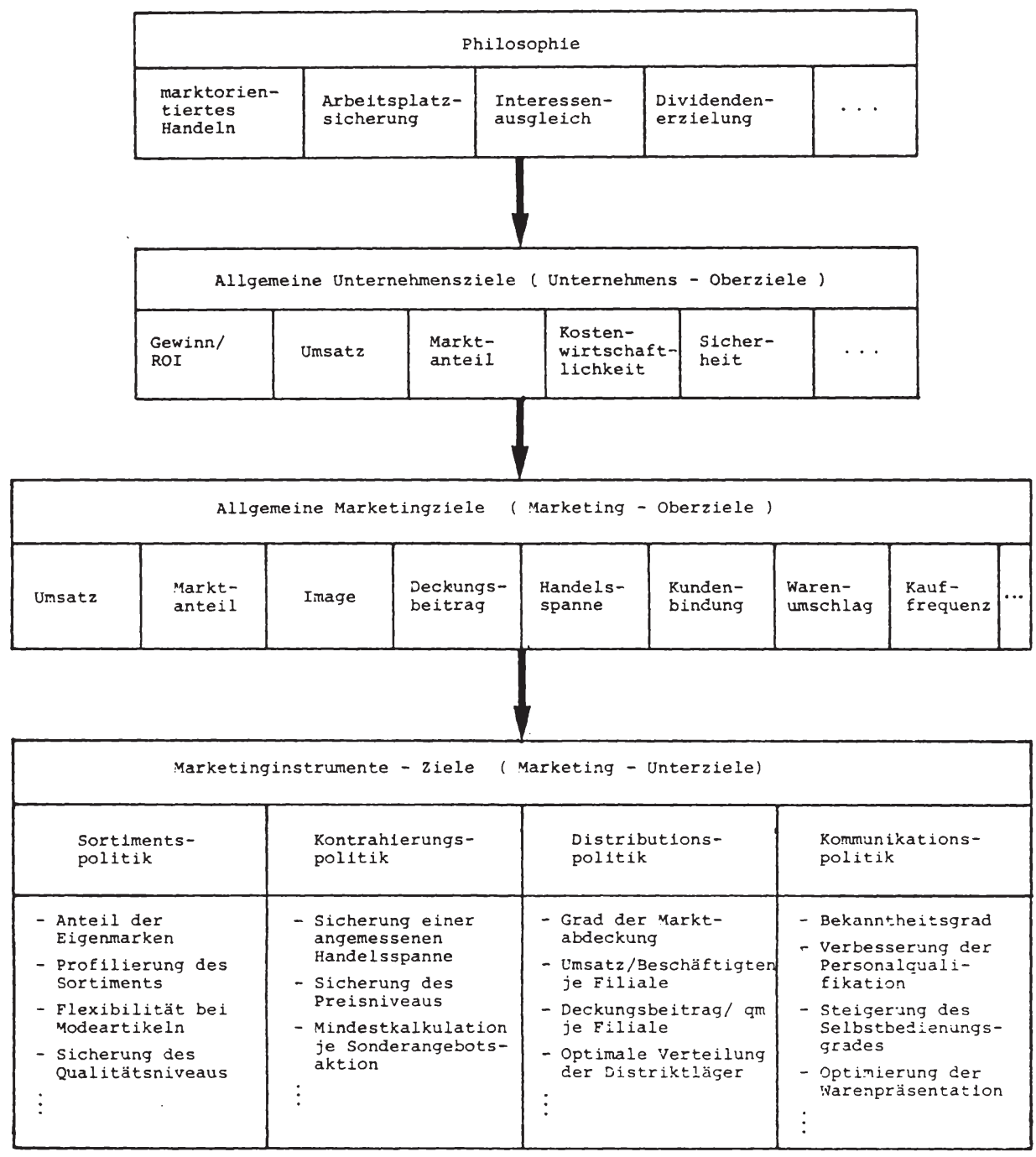

$\mathrm{Abb}$. 7: Beispielhaftes marketingorientiertes Zielsystem von Warenhäusern 
ketingplanung in Warenhäusern ist ein system von Steuerungsgrößen zu entwickeln, das die einzelnen Warengruppen- und Filial(gruppen)-Bereiche zielgerichtet im Sinne der Gesamtunternehmung koordiniert. Aufgrund der zentralen Stellung des Gewinnziels erscheint ein ROI-Kennzahlensystem ${ }^{1}$, das die Einflußgrößen der Kapitalrentabilität aufzeigt und es ermöglicht, hierarchische steuerungszahlen abzuleiten, die mit der marktorientierten organisationsStruktur der Warenhäuser in Einklang gebracht werden können, sinnvoll 2 .

Dieser organisatorische Aspekt der dualistischen Planung in Warenhäusern wird in der Abbildung 8 deutlich, die den Zusammenhang zwischen dem Umsatz und dem Betriebsergebnis im Hinblick auf die warengruppen- und die filialbezogene Analyse aufzeigt. Der Gesamtumsatz und das Gesamtergebnis des Warenhauses können zum einen auf der Basis der Warengruppen und zum anderen auf der Basis der Filialen ermittelt werden ${ }^{3}$.

1 Vgl. zur Darstellung und Diskussion des ROI-Kennzahlenschemas z.B. Aghte, K., a.a.O., S. 115 ff.; Bircher, B., a.a.O., S. 125; Koch, H., Aufbau der Unternehmensplanung, Wiesbaden 1977, S. $131 \mathrm{f}$. (im folgenden zitiert als Koch, H., Aufbau).

$2 \mathrm{Vgl}$. zur handelsspezifischen Interpretation des ROIKennzahlensystems Barth, K., Unternehmensführung, a.a.O., S. 162 ff..

3 Der Umsatz (U) ergibt sich aus den Warengruppenumsätzen oder den Filialumsätzen

$$
U=\sum_{i=1}^{n} W_{i} \quad \text { oder (2) } U=\sum_{j=1}^{m} F U_{j}
$$

Das Betriebsergebnis ergibt sich aus den Warengruppenerlösen (WGE) oder den Filialerlösen (FE) zusammen

$$
E=\sum_{i=1}^{n} \text { WGE oder }
$$$$
\text { (2) } \quad E=\sum_{j=1}^{m} F E_{j}
$$

Die Verknüpfung beider Bereiche erfolgt bei der Ergebnisermittlung der Warengruppen (WGE) und der Filialen

$$
\begin{aligned}
& \text { (1) } W_{i}=W_{i}-\sum_{\substack{i=1 \\
i=\text { const. }}}^{m} I K_{j / i} \\
& \text { wobei WGS = Warengruppenspanne } \\
& \text { (2) } \mathrm{FE}_{j}=\mathrm{FU}_{j}-\sum_{i=1}^{\mathrm{n}} \quad \mathrm{WGK}_{i / j} \\
& j=\text { const. }
\end{aligned}
$$

$\mathrm{FK}_{j / i}=$ alle Filialkosten der Warengruppe $i$

WGK $_{i / j}=$ alle Warengruppenkosten der Filiale $j$. 


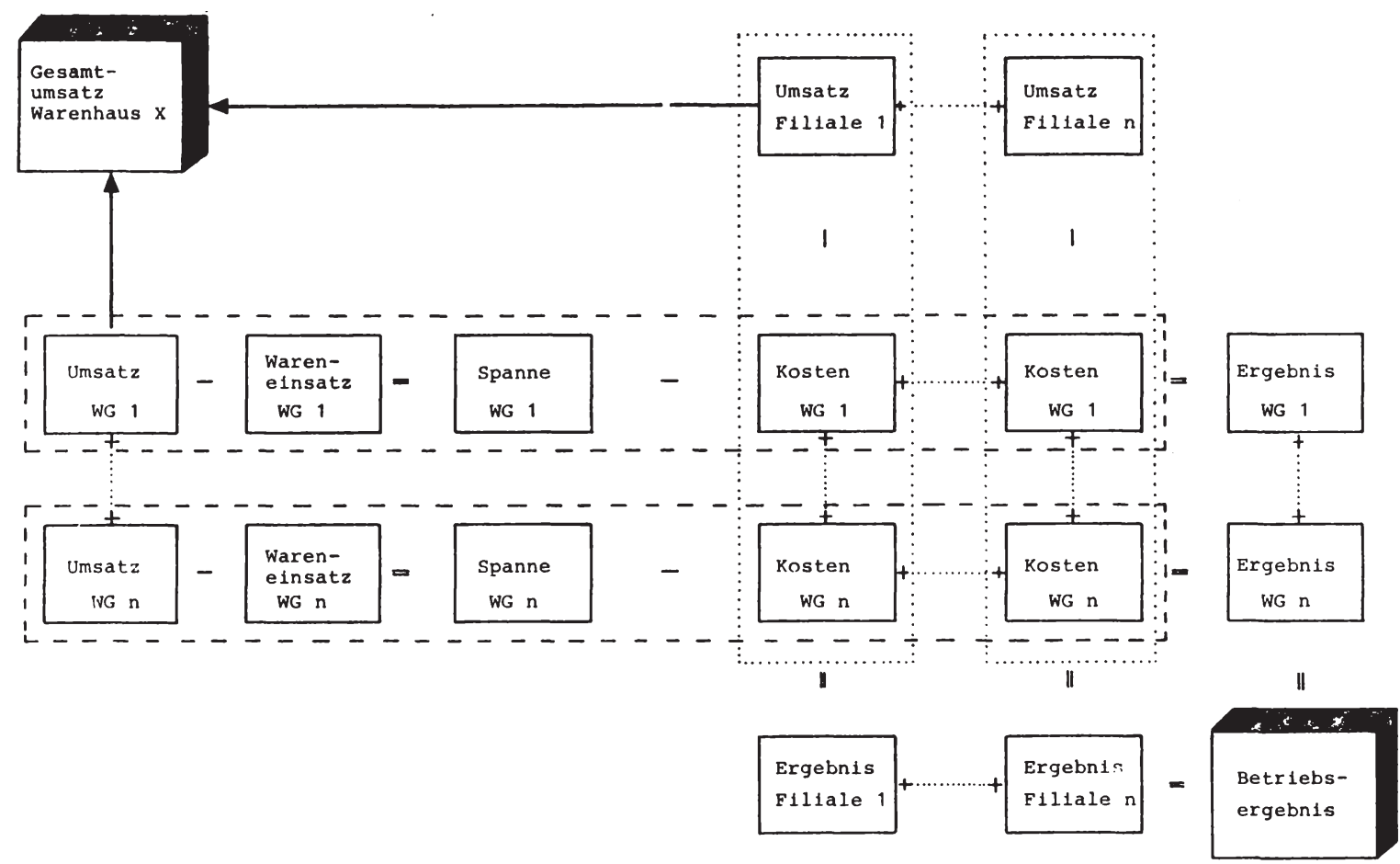

Abb. 8 : Zusammenhang zwischen Umsatz und Erfolgsziel bei dualistischer Planung 
Der enge Zusammenhang zwischen der Planung bzw. Organisation und der hierarchischen Struktur des Zielsystems macht die Notwendigkeit deutlich, die globalen Zielinhalte (vgl. Abbildung 9 ) eindeutig im Hinblick auf die relevanten Planungsobjekte (Gesamtunternehmung, einzelne Warenoder Filialgruppen) zu konkretisieren ${ }^{1}$. Denn nur in dieser eindeutigen Formulierung können die zielsetzungen in Warenhäusern als Steuerungsgrößen eingesetzt werden und ihre Koordinierungsfunktion erfüllen ${ }^{2}$.

Die aufgezeigte Struktur eines marketingorientierten Zielsystems in Warenhäusern enthebt die Entscheidungsträger nicht des Problems, die entscheidungsfeldbedingten Interdependenzrelationen sorgfältig $\mathrm{zu}$ analysieren. Die ermittelten Interdependenzen sind sowohl bei der $\mathrm{Ab}-$ leitung der Mittel-Zweck Hierarchie als auch bei der Formulierung spezifischer Leistungs- bzw. Steuerungskennzahlen zu berücksichtigen ${ }^{3}$.

Als Beispiele für eine derartige Interdependenzanalyse ist in der Abbildung 9 der Umsatz eines Warenhauses ${ }^{4}$ in seiner Beziehung zu einigen ausgewählten Marketingzielen dargestellt. Dabei sind die komplementären mit (+), die konkurrierenden mit (-) und die neutralen zielbeziehungen mit (o) gekennzeichnet.

1 Zur Problematik der Bildung von Zielsystemen bei dezentraler Organisationsstruktur vgl, auch Berthel, J., a.a.O., S. $87 \mathrm{ff}$.

2 Greift man sich als Beispiel das Marktanteilsziel heraus, so sind folgende Formulierungen denkbar:

Marktanteil Gesamtunternehmung $=2,6 \%$; Marktanteil der Warengruppe $\mathrm{x}$ überregional $=6,8 \%$; Marktanteil der Filiale $\mathrm{Y}$ im Einzugsgebiet $=4,5 \%$; Marktanteil der Warengruppe $\mathrm{X}$ im Einzugsgebiet der Filiale $\mathrm{Y}=8,2 \%$.

3 Eine Möglichkeit, diese Interdependenzen festzustellen und auch zu quantifizieren stellt eine handelsspezifische PIMS-Studie dar (vgl, auch Kapitel 1.3 der Arbeit). Sie würde es ermöglichen, die wesentlichen internen und externen Variablen (Zielgrößen) zu erkennen, die den ROI eines Warenhauses beeinflussen.

4 Auf eine Differenzierung nach Warengruppen oder Filialen wird hier verzichtet. 
Eine positive Beeinflussung des umsatzes ist u.a. durch die Ziele "Sicherung der Kundentreue" sowie "Erschließung neuer Käuferschichten" gegeben ${ }^{1}$. Die Sicherung der Kundentreue wird ihrerseits von der zielerreichung der Sortimentsprofilierung sowie der Imageverbesserung positiv beeinflußt. Eine nur partiell geltende Komplementarität ist zum Ziel der Handelsspannensicherung gegeben. Für den Fall, daß dieser zielsetzung höhere priorität zukommt mit der Konsequenz einer hohen Preisfestsetzung, ist ein überproportionaler Verlust der bisherigen Kunden denkbar.

Das Ziel der Erschließung neuer Käuferschichten wird umso eher erreichbar sein, je profilierter das Sortiment, je positiver das Image und je höher der Bekanntheitsgrad des Warenhauses insgesamt ist. Eine neutrale Beziehung ist zum Ziel der Handelsspannensicherung gegeben.

Das Ziel der Sortimentsprofilierung wird u.a. vom Qualitätsniveau positiv beeinfluBt. Der Eigenmarkenanteil profiliert einerseits das Warenhaus, indem er die Leistungsfähigkeit demonstriert, andererseits darf er auch nicht zu hoch sein, da ein profiliertes Sortiment sich auch durch das Führen klassischer Herstellermarken auszeichnet ${ }^{2}$. Ebenso wird ein gehobenes Preisniveau und/oder die Erhöhung der Zahl der Sonderangebote nur in bestimmten Zielerreichungsgraden zur Profilierung beitragen. Der Bekanntheitsgrad verhält sich zur Sortimentsprofilierung neutral.

1 Selbstverständlich sind hier noch weitere zielinhalte wie z.B. die Kauffrequenz zu nennen.

2 Dies stellt im Grunde ein nahezu unlösbares optimierungsproblem dar. 
Das Ziel der Imageverbesserung wird durch ein hohes Qualitätsniveau, einen hohen Bekanntheitsgrad und ein mittleres/gehobenes Preisniveau positiv beeinflußt. Wiederum nur partiell kompatibel sind die zielsetzungen "Eigenmarkenanteil" sowie "Erhöhung der Zahl der Sonderangebote".

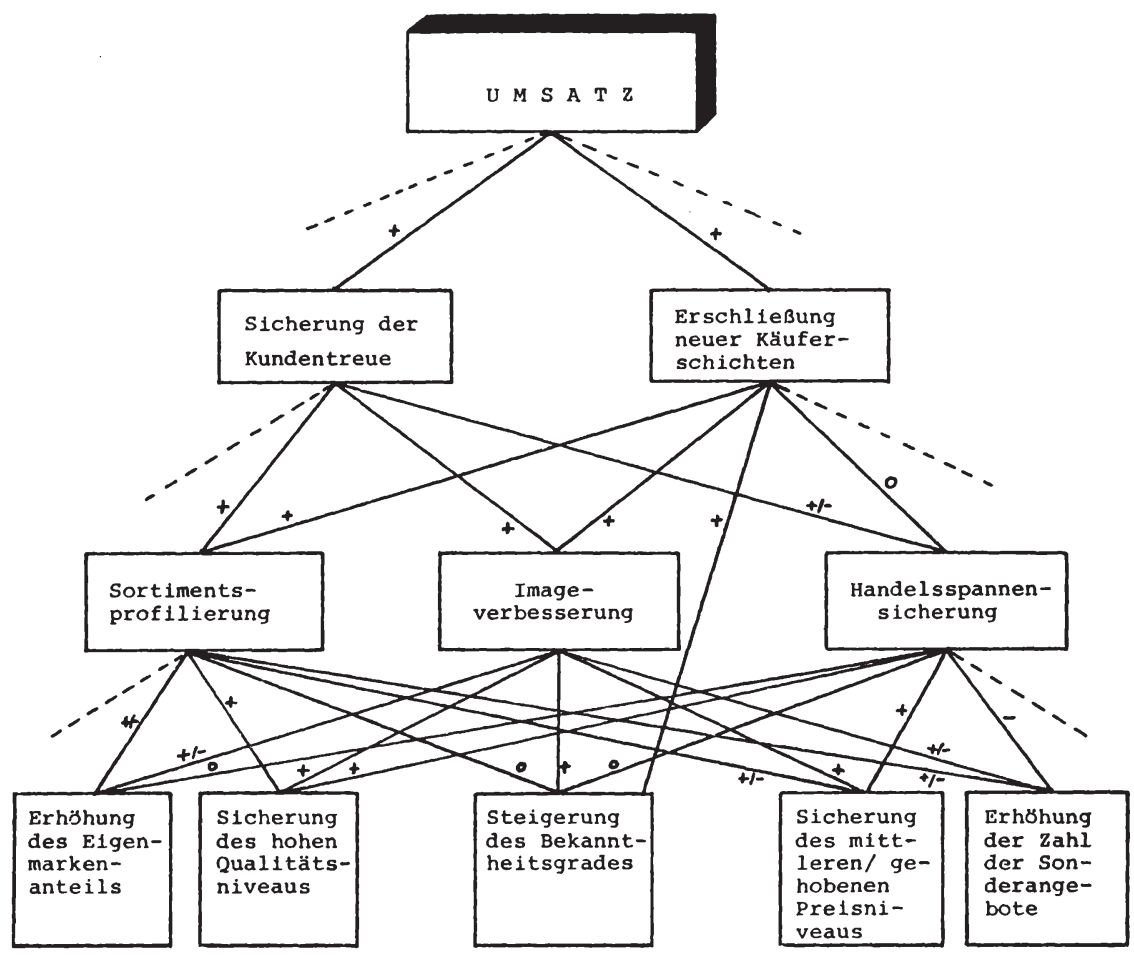

Abb. 9 : Ausschnitt eines marketingorientierten Zielsystems von Warenhäusern 
Die Handelsspannensicherung weist zur Zielsetzung "Qualitätsniveau-" und "Preisniveau-Sicherung" eine kompatible Beziehung auf. Zur Zielsetzung des Eigenmarkenanteils sowie des Bekanntheitsgrades sind neutrale Beziehungen vorhanden.

Die Erhöhung der Zahl der Sonderangebote wird sich auf das Ziel der Handelsspannensicherung negativ auswirken, da diese Politik ja definitionsgemäß durch einen unterdurchschnittlich kalkulierten Verkaufspreis gekennzeichnet ist.

Insbesondere die Diskussion der nach der Ziel-Mittel-Relation gebildeten Zielsysteme zeigt exemplarisch das Dilemma der strategischen Planung auf. Einerseits bilden die Ziele die Voraussetzung, um Strategien entwickeln zu können. Andererseits können sie erst nach einer Abschätzung der strategischen Möglichkeiten soweit konkretisiert werden, daB sie die beschriebenen Funktionen erfüllen können ${ }^{1}$. Dabei müssen die strategischen Verhaltensweisen keineswegs vollständig und detailliert bekannt sein. Vielmehr reichen in der realen planungsituation oftmals bereits grobe Vorstellungen aus. So kommt im Rahmen der strategischen Marketingplanung in Warenhäusern insbesondere den Positionierungsentscheidungen, die eine globale Beschreibung der Stellung im Markt zum Gegenstand haben, eine solche zielkonkretisierende Funktion $z u$. Dabei muß die Positionierung im Sinne einer kundenorientierten Unternehmensführung zunächst im Hinblick auf die zu bearbeitenden Käufersegmente vorgenommen werden.

1 In diesem zusammenhang wird in der Literatur auch oft von "Zielen vor Planung" und "Zielen nach Planung" gesprochen. Vgl. hierzu z.B. Töpfer, A., a.a.O., S.131 ff.; Wild, J., Grundlagen, a.a.O., S. $52 \mathrm{ff} .$. 


\subsection{Positionierung von Warenhäusern im Hinblick auf Käufersegmente}

Die Basis für die Positionierung eines Warenhauses im Hinblick auf die verschiedenen Käufersegmente legt das Konzept der Marktsegmentierung nahe ${ }^{1}$. Die Notwendigkeit zur Segmentierung des Marktes ergibt sich für Warenhäuser aus der Heterogenität der Konsumentenanforderungen an Einzelhandelsleistungen einerseits und aus der Heterogenität des Leistungsangebots der Einzelhändler andererseits. Damit ist es die primäre Aufgabe des Warenhausmanagement, Konsumentengruppen zu finden, "... deren Anforderungen an bestimmte Verhaltens- und Strukturmerkmale der Anbieter gleichgerichtet sind" 2 . Es besteht weitgehender Konsens in Theorie und Praxis, daß die richtige Wahl der Zielgruppe(n) die Weichen für den Erfolg einer spezifischen Einzelhandelskonzeption stellt ${ }^{3}$ und damit auch für Warenhäuser eine strategische Rahmenentscheidung darstellt.

1 Vgl. zur Darstellung des Konzepts z.B. Ahlert, D., Marketing, a.a.0., S. 41 ff.; Derselbe, Probleme der Abnehmerselektion und der differenzierten Absatzpolitik auf der Grundlage der segmentierenden Markterfassung, in: Der Markt 1973, Nr. 4, S. 103 ff.; Bauer, E., Markt-Segmentierung, Stuttgart 1977; Böhler, H., Methoden und Modelle der Marktsegmentierung, Stuttgart 1977; Crone, G., Marktsegmentierung. Eine Analyse zur Zielgruppendefinition unter besonderer Berücksichtigung soziologischer und psychologischer Kriterien, Frankfurt/Bern 1977; Brumm, G., Die Problematik der Marktsegmentierungspolitik im Rahmen des Marketing, Diss. Berlin 1973; Gröne, A., Marktsegmentierung bei Investitionsgütern, Wiesbaden 1977, insbes. S. 3o ff.; Groh, G., Marktsegmentierung, in: Handwörterbuch der Absatzwirtschaft, Hrsg. Tietz, B., Stuttgart 1974, Sp. 1408-1420; Krautter, J., Zum Problem der optimalen Marktsegmentierung, in: $\mathrm{ZfB} 1975, \mathrm{~S} .109-$ 128; Meffert, H., Marketing, a.a.o., S. $213 \mathrm{ff..}$

2 Hansen, U., Marketing, Band I, a.a.O., S. 59.

$3 \mathrm{Vgl.hierzu} \mathrm{z.B.} \mathrm{die} \mathrm{Aussagen} \mathrm{bei} \mathrm{Bearchell,} \mathrm{C.A.,}$ a.a.O., S. 37 ff.; Bermann, B., Evans, J.R., a.a.O., S. 102 ff.; Davidson et al., a.a.0., S. $105 \mathrm{ff.}$. 
Das Marktsegmentierungskonzept beinhaltet insgesamt ärei Stufen (vgl. Abbildung 10):

1. die Aufspaltung des Marktes in Marktsegmente (Markterfassung)

2. die Auswahl der zu bearbeitenden Segmente (Segmentselektion) und

3. die Entwicklung geeigneter Marktbearbeitungsstrategien, die im Rahmen der Marketing-Instrumentalstrategien erfolgt.

Das ziel der Markterfassung ist es, intern homogene und extern heterogene Marktsegmente zu bilden, an cie folgende Anforderungen ${ }^{1}$ zu stellen sind: Ansprechbarkeit, Zugänglichkeit und Wirtschaftlichkeit der differenzierten Segmentbearbeitung, Akzeptanz durch die Mitarbeiter sowie zeitliche Stabilität ${ }^{2}$. Die Markterfassung erfolqt auch für Warenhäuser auf der Basis sozio-demographischer (Alter, Einkommen, Beruf, Geschlecht, Wohnort, Haushaltsgröße usw.) und psychographischer Kriterien (Einstellungen, Lebensstil, Persönlichkeitsmerkmale, Kaufgewohnheiten usw. $)^{3}$.

$1 \mathrm{Vgl}$. auch den Literaturüberblick bei: Kuhlmann, E., Die Selektion von Segmentierungsmerkmalen, Arbeitspapiere zum Marketing, Bd. 4, Hrsg.: Engelhardt, H.W., Hammann, P., Bochum 1979, S. 3 ff..

2 Hierbei werden diese Anforderungen auf die Segmente bezogen, während in der Literatur diese Anforderungen oftmals an die Segmentierungskriterien gestellt werden. Vgl. hierzu Gröne, A., a.a.O., S. $43 \mathrm{ff..}$

$3 \mathrm{Vgl}$. hierzu den Überblick bei Freter, H., Marktpositionierung, Habilitationsschrift Münster 1978, S. 17; vgl. auch Kaiser, A., Die Identifikation von Marktsegmenten, Berlin 1978, S. $35 \mathrm{ff..} \mathrm{Dabei} \mathrm{rücken} \mathrm{in} \mathrm{jüngster}$ Zeit insbesondere auch Aspekte der Familienkaufentscheidung in den Vordergrund. Vgl. hierzu z.B. Dahlhoff, H.-D., Kaufentscheidungsprozesse von Familien, Frankfurt 1980; Meffert, H., Dahlhoff, H.-D., Kollektive Kaufentscheidungsprozesse von Konsumenten, in: Handelsforschung heute, Festschrift zum 5ojährigen Bestehen der Forschungsstelle für den Handel, Berlin 1979, S. 193-205. 


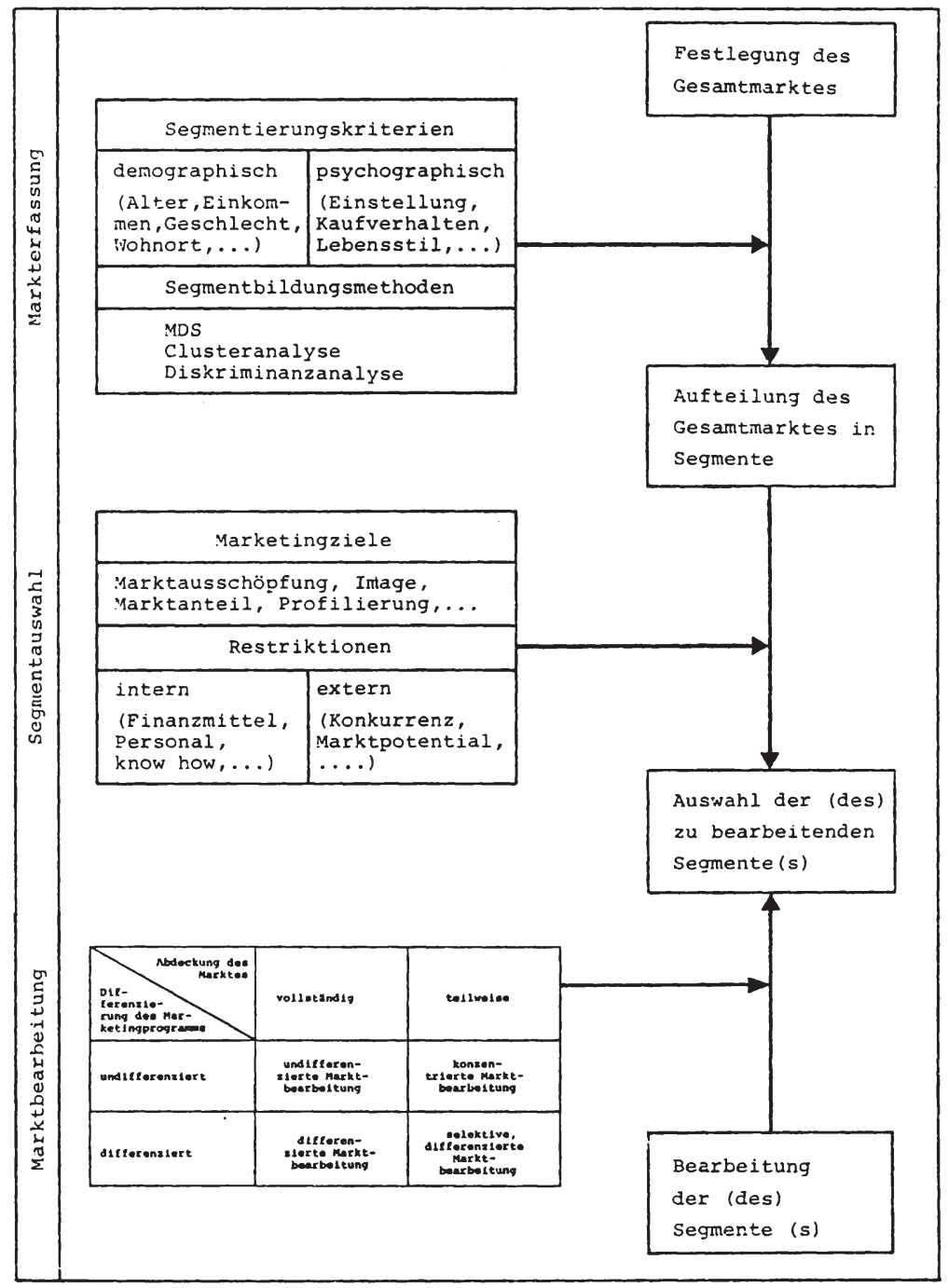

Abb.10: Grundstruktur des Marktsegmentierungs-Konzepts 


\subsection{Demographische und psychographische Struktur der}

Zielgruppen von Warenhäusern

Analysiert man die Stellung der Warenhäuser innerhalb der Betriebsformen des Einzelhandels, so wird deutlich, daß sie sich weniger auf eine oder wenige zielgruppen beschränken, sondern vielmehr viele unterschiedliche Verbrauchersegmente bearbeiten wollen. Dies belegt die demographische Struktur der Warenhauskunden und -nichtkunden in Tabelle 41 . Demnach sind Warenhaus-Kunden überwiegend

- zwischen 14 und 69 Jahren alt und

- zumeist verheiratet

- besitzen Grund- und Mittelschulbildung

- entstammen zumeist Angestellten- und Arbeiterberufen

- weisen ein mittleres Haushalts-Nettoeinkommen (ca. 1000 - 2000 DM) auf.

Damit entspricht die demographische struktur der Warenhauskunden ${ }^{2}$ weitgehend der durchschnittlichen Schichtung der Bevölkerung in den genannten Merkmalen.

Vermutet man im Hinblick auf das Einkommen zunächst eine große Bedeutung der einkommensschwächeren Bevölkerungsschichten für Warenhäuser, so zeigt diese Analyse einen Schwerpunkt der Warenhauskunden in mittleren Einkommens-

1 Die hier wiedergegebenen Zahlenangaben sind der Zielgruppenanalyse eines Warenhauskonzerns entnommen, die repräsentativ für die Bundesrepublik 1972 durchgeführt wurde. RegelmäBige Nachfolgeuntersuchungen zeigen, daß - bis auf die Wertangaben der Nettoeinkommen - die festgestellten Angaben auch heute noch stimmen.

2 Eine zusätzliche Analyse der Abweichungen in den Merkmalen zwischen den vier großen Warenhauskonzernen ergab keine signifikanten Unterschiede. Insofern ist es gerechtfertigt, allgemeingültig - ohne firmenspezifische Ausrichtung - von Warenhauszielgruppen zu sprechen. 


\begin{tabular}{|c|c|c|}
\hline Kriterien & Warenhauskunden & $\begin{array}{l}\text { Nichtwaren- } \\
\text { hauskunden }\end{array}$ \\
\hline $\begin{array}{l}\text { Geschlecht } \\
\text { - männlich } \\
\text { - weiblich }\end{array}$ & $\begin{array}{l}47 \\
53\end{array}$ & $\begin{array}{l}54 \\
46\end{array}$ \\
\hline $\begin{array}{l}\frac{\text { Alter }}{14-29} \text { Jahre } \\
30-490 " \\
50-69 " \\
70 \text { Jahre und älter }\end{array}$ & $\begin{array}{r}23 \\
39 \\
28 \\
9\end{array}$ & $\begin{array}{l}15 \\
32 \\
34 \\
19\end{array}$ \\
\hline $\begin{array}{l}\text { Familienstand } \\
\text { verheiratet } \\
\text { ledig } \\
\text { verwitwet/geschieden }\end{array}$ & $\begin{array}{l}70 \\
18 \\
12\end{array}$ & $\begin{array}{l}63 \\
16 \\
21\end{array}$ \\
\hline $\begin{array}{l}\text { Schulbildung } \\
\text { Volksschule } \\
\text { weiterführ. Schulen } \\
\text { (ohne Abiturienten) } \\
\text { Abitur } \\
\text { Hochschule, Uni }\end{array}$ & $\begin{array}{r}67 \\
23 \\
4 \\
5\end{array}$ & $\begin{array}{r}77 \\
15 \\
3 \\
4\end{array}$ \\
\hline $\begin{array}{l}\text { Beruf } \\
\text { Selbständige, incl. } \\
\text { Landwirte } \\
\text { Angestellte } \\
\text { Beamte } \\
\text { Arbeiter } \\
\text { in Ausbildung } \\
\text { Hausfrau }\end{array}$ & $\begin{array}{r}8 \\
24 \\
5 \\
20 \\
8 \\
28\end{array}$ & $\begin{array}{r}13 \\
17 \\
4 \\
26 \\
5 \\
25\end{array}$ \\
\hline $\begin{array}{l}\text { Beruf des Haushaltungs- } \\
\text { vorstands } \\
\text { Selbständige, incl. } \\
\text { Landwirte } \\
\text { Angestellte } \\
\text { Beamte } \\
\text { Arbeiter } \\
\text { in Ausbildung }\end{array}$ & $\begin{array}{r}15 \\
34 \\
10 \\
37 \\
0\end{array}$ & $\begin{array}{r}21 \\
26 \\
8 \\
40 \\
0\end{array}$ \\
\hline $\begin{array}{l}\frac{\text { Kinder im Haushalt }}{\text { bis zu } 5 \text { Jahren }} \\
6-13 \text { Jahre }\end{array}$ & $\begin{array}{l}21 \\
26\end{array}$ & $\begin{array}{l}15 \\
21\end{array}$ \\
\hline $\begin{array}{l}\text { Haushalts-Nettoein- } \\
\frac{\text { kommen }}{\text { bis } 799} \text { DM } \\
800-999 \text { DM } \\
1000-1499 \text { DM } \\
1500-1999 \text { DM } \\
2000-2499 \text { DM } \\
2500 \text { DM und mehr }\end{array}$ & $\begin{array}{l}11 \\
10 \\
28 \\
23 \\
15 \\
12\end{array}$ & $\begin{array}{l}21 \\
11 \\
24 \\
19 \\
12 \\
11\end{array}$ \\
\hline
\end{tabular}

Tab. 4 : Demographische Beschreibung von Warenhauskunden und Nichtwarenhauskundern 
klassen und einen beachtlichen Anteil von Käufern, die den gehobeneren Klassen (2000 DM und mehr) zuzurechnen sind (27\%). Diese Tatsache läßt sich u.a. damit erklären, daß hohe Bedarfsansprüche nicht unbedingt mit hohem Einkommen korreliert sind, wogegen diese Beziehung in den unteren Einkommensklassen wesentlich enger ist. Damit bestärkt dieses Ergebnis die häufiger zu findende These, daß sich für Warenhauskunden eine exakte Abgrenzung des Einkommens nach oben nicht durchführen läßt. Ob im Warenhaus gekauft wird oder nicht, hängt von den angebotenen Warengruppen bzw. Artikeln $a b$. Allerdings dürfte der Kunde mit einem Einkommen von mehr als 2500 DM aufgrund seiner hohen Bedarfsansprüche bislang kaum als typischer Warenhauskunde zu bezeichnen sein.

Die Nicht-Warenhauskunden setzen sich vor allem

- aus älteren Leuten (19\% über 70 Jahre)

- aus Beziehern unterdurchschnittlicher Einkommen (teils alters-, teils ausbildungsbedingt)

- aus Mitgliedern von Selbständigen- und Landwirtenhaushalten (21\%)

zusammen. Damit unterscheiden sie sich in ihrer demographischen Struktur sehr viel stärker von der Gesamtbevölkerung als die Warenhauskunden.

Insgesamt bietet die demographische Käuferanalyse für das Warenhaus kaum Ansatzpunkte zur Definition von Zielgruppen, die sich mit spezifischem Marketing-Instrumenteeinsatz differenziert bearbeiten lassen ${ }^{1}$. Deshalb ist der Rückgriff auf psychographische Merkmale notwendig.

$1 \mathrm{Zu}$ diesem Ergebnis kommt auch Steffenhagen. Vgl. Steffenhagen, H., Marktführer, a.a.O., S. 24 . 
Die Beschreibung der Warenhauskunden anhand psychographischer Variablen wie z.B. Persönlichkeit, Lebensstil, Einstellungen stellt im Hinblick auf die Erfassung allerdings ein ungleich schwierigeres problem als die demographische Beschreibung dar.

Im Rahmen einer psychographischen Zielgruppenanalyse für ein Warenhaus wurde die Käuferschaft anhand der Dimensionen "Qualitätsempfinden", "Aufgeschlossenheit für neue Produkte" und "Prestige-Bedürfnis" typologisiert". Dabei war zu prüfen, ob sich die Warenhauskunden hinsichtlich dieser Merkmale voneinander und von den Nichtwarenhauskunden unterscheiden (vgl. hierzu Abbildung 11).

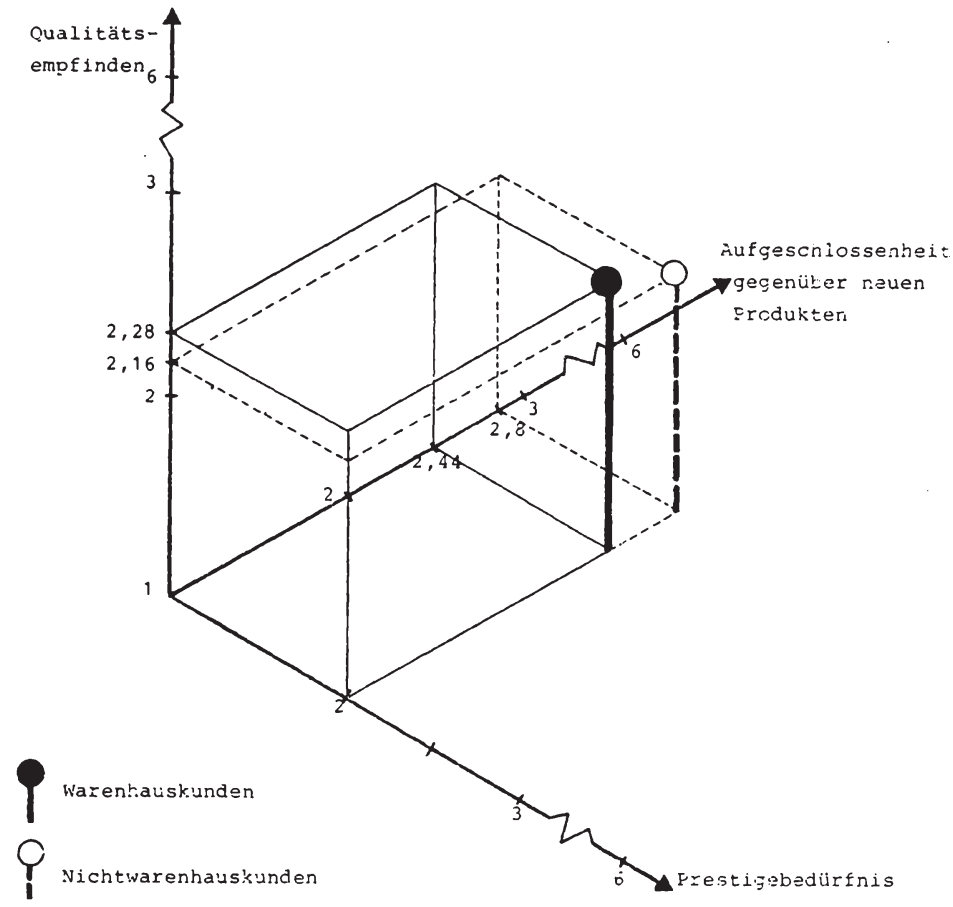

Abb. 11: Dreidimensionales Positionierungsmodell von

(Nicht-) Warenhauskunden auf der Basis psychographischer Variablen

1 Dieser Ansatz wurde der Analyse eines Warenhauskonzerns zugrundegelegt, der die folgenden Daten entnommen sind. 
Die Dimensionen wurden mit Hilfe mehrerer Kriterien gebildet, die den Befragten in Form von Statements vorgelegt wurden, denen sie mittels einer 5er-Skala von 1 (= volle Zustimmung) bis 6 (= volle Verneinung) mehr oder weniger zustimmen konnten ${ }^{1}$. Nach den Ergebnissen dieser Untersuchung unterscheiden sich die Warenhauskunden besonders in den Dimensionen "Qualitätsempfinden" von den Nichtwarenhauskunden (Mittelwert 1,84:2,2); sie akzeptieren schlechte Qualität in keinem Fall. Nichtwarenhauskunden sehen einen deutlich ausgeprägten Preis-Qualitäts-Zusammenhang und sind durchaus bereit, für Qualität einen höheren Preis zu bezahlen. Bezüglich der Aufgeschlossenheit für neue Produkte zeigen sich die Nichtwarenhauskunden deutlich konservativer. Sie sind weniger probierfreudig und stützen sich bei Kaufentscheidungen lieber auf ihre Erfahrungen. Die Analyse des Prestige-Bedürfnisses zeigt kaum Unterschiede zwischen den beiden Gruppen.

Ein weiterer Ansatz befaßt sich mit der Typologisierung ${ }^{2}$ der Käufer im Betriebsformenvergleich anhand der Dimensionen "Freude am Einkaufen" und "Niveauorientierung"3 . Die Abbildung 12 zeigt als Ergebnis die Abgrenzung derjenigen Konsumenten, die das Warenhaus, den Verbrauchermarkt oder das Fachgeschäft präferieren. Danach existiert "... für die Warenhäuser ein relativ großes, potentielles Käuferpublikum als tragfähige Basis eines Markterfolges"A.

1 So wurde z.B. die Dimension "Aufgeschlossenheit für neue Produkte" mit folgenden statements erfaBt:

- probiere gern neue Produkte

- Angebot zu groß, kaufe lieber Bekanntes

- Vielfalt fasziniert, probieren macht SpaB

- kaufe nur bekannte oder empfohlene Produkte

- prinzipiell skeptisch qegenüber neuen Produkten.

2 Vgl. zur Bildung von Typologien z.B. Dichtl, E., Die Bildung von Konsumententypologien als Grundlage der Marktsegmentierung, in: Wist, 3. Jg. (1979), Heft 2, S. 54-59.

$3 \mathrm{Vgl.}$ Steffenhagen, H., Marktführer, a.a.O., S. 24. Leider werden zur MeBmethode keinerlei Angaben gemacht.

4 Ebenda. 


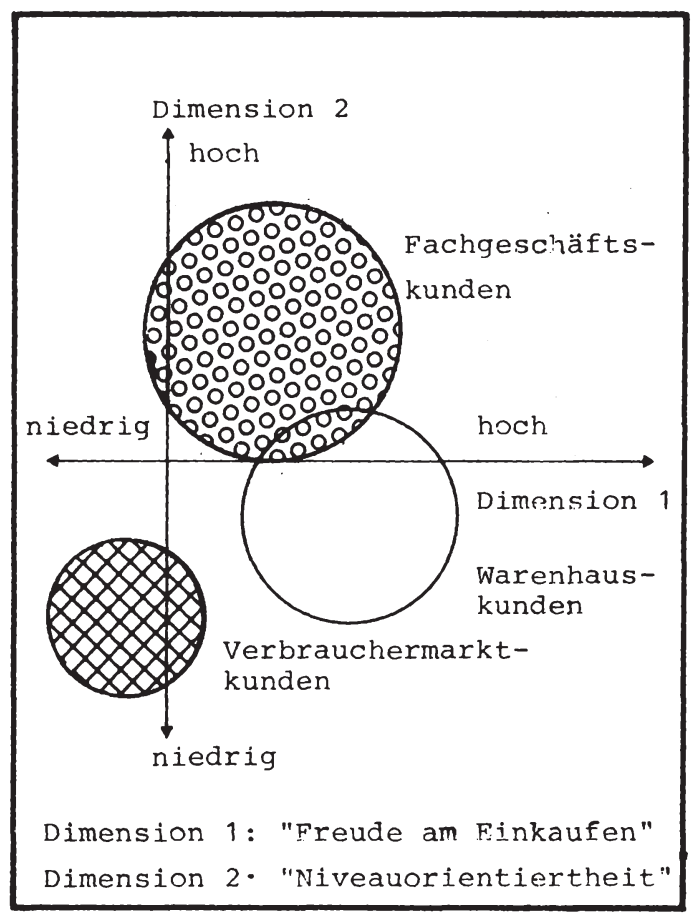

$\begin{aligned} \text { Abb. } 12: & \text { Typologisierung von Kunden } \\ & \text { ausgewählter Betriebsformen }\end{aligned}$

Diese psychographischen Charakterisierungen von Warenhauskunden stellen vielversprechende Ansätze zur Angrenzung von Marktsegmenten bzw. Zielgruppen im Warenhaus dar, da sie die kaufverhaltensbestimmenden Elemente berücksichtigen. Allerdings liegt in diesen Darstellungen die Gefahr von Fehlinterpretationen in der Hinsicht, daß Warenhauskunden in jedem Fall z.B. weniger qualitätsorientiert und weniger konservativ sind, und zum anderen, daß ver- 
brauchermarktkunden generell nicht im Warenhaus oder Fachgeschäft einkaufen. Eine weitere Problematik dieser Untersuchungen liegt in der fehlenden Differenzierung nach Warengruppen bzw. nach standorten.

Als Fazit aller Studien ${ }^{1}$ ist festzuhalten, daß die Warenhäuser bislang eine "Zielgruppenstrategie der gehobenen Mitte" verfolgen. Dabei wird diese breite Zielgruppe ${ }^{2}$ von allen (deutschen) Warenhäusern - abgesehen von kleinen Unterschieden- umworben. Angesichts dieser Situation muß sich die Warenhausleitung vor dem Hintergrund der gegenwärtigen "Warenhauskrise" und dem Erfordernis der eindeutigen Positionierung die Frage nach der Notwendigkeit und den Möglichkeiten der Änderung der bisherigen Zielgruppenstrategie stellen, womit der zweite Aspekt der Marktsegmentierung, die Segmentselektion angesprochen ist.

1 In den letzten Jahren rücken Imageanalysen, die versuchen, die Präferenz der Käufer für bestimmte Betriebsformen herauszufinden, zunehmend in den Vordergrund. Vgl. 2.B. Anders, H.J., Analyse des Einkaufsverhaltens, in: Der Einzelhandel 1978/79. Versuch einer Standortbestimmung, Hrsg.: Gesellschaft für Konsum-, Markt- und Absatzforschung e.v. (GfK) Nürnberg 1978, S.9ff.; BBE (Hrsg.), Untersuchung über die Einstellung der BadenWürttembergischen Bevölkerung zu ausgewählten Betriebsformen, Stuttgart 1977, insbesondere S. $133 \mathrm{ff.;}$ Tietz, B., Wie Verbraucher über Einzelhandelsleistung denken, in: ASW 3/1978, S. 47-53 (im folgenden zitiert als: Tietz, B., Einzelhandelsleistung).

Die Ergebnisse dieser Untersuchungen können zwar unterstützend zur Abgrenzung von Kundensegmenten anhand psychographischer Variablen herangezogen werden, ihr eigentlicher Wert liegt jedoch im Aufdecken spezifischer Stärken und Schwächen eines Warenhauses gegenüber den Konkurrenten.

2 Die solchermaßen für deutsche Warenhäuser grob umrissene Zielgruppe unterscheidet sich nur marginal von der Zielgruppendefinition der schweizerischen Warenhausgesellschaft "Globus", die folgendermaßen lautet:

- jüngere und mittlere Altersklasse

- mittlere Kaufkraftklasse

- preis - und qualitätsbewußt

- moderne Menschen mit Freude am Einkaufen.

Vgl. Bitterli, R.E., Aktuelle Marketingprobleme des Warenhauses, in: Entwicklungstendenzen im Handel, hrsg. v. J.S. Krul is-Randa, Bern/Stuttgart 1979, S. 83-89. 


\subsection{Zielgruppenstrategien von Warenhäusern}

Bei der Auswahl der Zielgruppen hat sich die Warenhausleitung primär an den zukünftigen Beiträgen der einzelnen Segmente zur Erreichung der gesetzten Marketingziele zu orientieren. Hierbei sind sowohl quantitative Ziele (Umsatz, Marktausschöpfung, Deckungsbeitrag, Kosten usw.) als auch qualitative ziele (Image, Profilierung, Verträglichkeit mit anderen Segmenten usw.) heranzuziehen.

Wie jedes Einzelhandelsunternehmen erstreckt sich das Entscheidungsspektrum der Warenhäuser bezüglich der Zielgruppenwahl von einer exklusiven bis zu einer extensiven Zielgruppenstrategie (vgl. Abbildung 13 ).

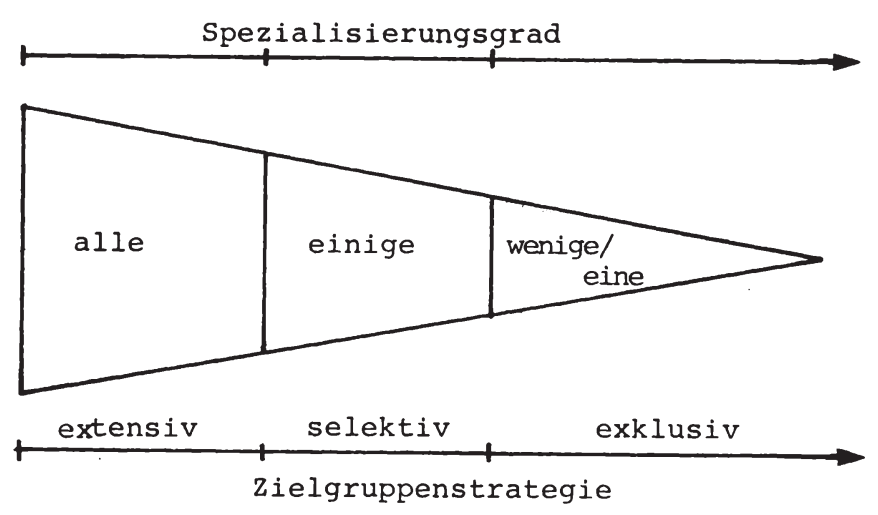

Abb. 13 : Entscheidungsspektrum Zielgruppenstrategie

Blickt man auf die demographische und psychographische Beschreibung der Warenhauskunden, so belegt diese eindeutig einen niedrigen Spezialisierungsgrad bzw. eine extensive Zielgruppenstrategie der Warenhäuser. Dies liegt in der 
Vielzahl der Warengruppen begründet, die diverse, mehr oder weniger heterogene Kundensegmente ansprechen, so daß sich eine starke Beschränkung auf einen bestimmten Teil der Kundensegmente aufgrund der strategischen Sortimentsentscheidung verbietet. Von daher stellt sich das Problem der Zielgruppenwahl bzw. -strategie für Warenhäuser im Rahmen der strategischen Grobplanung weniger in der grundsätzlichen Neuorientierung der Basisstrategie. Vielmehr läßt sich das Problem auf die Kernfrage nach der Ergänzung bzw. Abrundung des breiten Basissegments um Randgruppen reduzieren. ${ }^{1}$ In diesem Zusammenhang ist zu überprüfen, welche Randgruppen für Warenhäuser prinzipiell bearbeitungswürdig erscheinen.

Dabei ist zum einen das ziel der Kundenfrequenzerhöhung mit den Ausprägungen Gewinnung von Nichtwarenhauskunden, Gewinnung von Kunden der Warenhauskonkurrenz und Erhöhung der Kundentreue der bisherigen Kunden heranzuziehen. Zum anderen ist zu berücksichtigen, daß die einheitliche Gesamtkonzeption für das bisherige Basissegment nicht wesentlich beeinträchtigt werden darf, die Bearbeitung der ausgewählten Randsegmente lohnend sein muß und die Prioritäten in marketingstrategischen Uberlegungen umsetzbar sein müssen. Schlieblich ist erforderlich, daB die potentiellen Kunden eine aufgeschlossene Einstellung zum Leistungsprogramm der Warenhäuser erkennen lassen.

Unter Berücksichtigung dieser Selektionskriterien und der aktuellen demographischen Struktur der Warenhauskunden sind z.B. folgende drei Randsegmente beachtenswert: der sog. Altenmarkt (über 65 Jahre), die Haushalte mit niedrigem Einkommen (unter 1000 DM) und die Haushalte mit sehr hohem Einkommen (über 2500 DM). Für jedes dieser drei (bisherigen) Randsegmente ist nun mittels einer qualitativen Vorteils-Nachteils-Betrachtung die Vor-

1 Dabei ist es im realen Anwendungsfall durchaus denkbar, neben der globalen Betrachtung des Basissegments für den gesamten Warenhauskonzern eine differenzierte evtl. auch regional begrenzte abteilungsspezifische Segmentierung ergänzend heranzuziehen. 
teilhaftigkeit einer Bearbeitung und damit der Ausweitung der Basiszielgruppe zu überprüfen. Dabei sind Beurteilungskriterien wie z.B. Segmentgröße, Entwicklung, Kaufkraft, Warenhauseinstellung, Qualitäts-, Auswahl-, Bedienungs-, Preisansprüche usw. detailliert zu bewerten. Führt die Warenhausleitung diese Analyse für alle zur Entscheidung stehenden Segmente durch, lassen sich konkrete Hinweise für eine Neuorientierung in der zielgruppenstruktur erarbeiten.

Neben der Gewinnung neuer Randgruppen muB sich die Zielgruppenstrategie der Warenhäuser insbesondere am Ziel der Erhöhung der Kundenfrequenz orientieren. Damit sind die Schwerpunkte der Aktivitäten im Bereich des Basissegments zu legen, um die aktuellen Kunden zu veranlassen, häufiger im eigenen Warenhaus zu kaufen. Im Zuge der zunehmenden Heterogenisierung der Kundenwünsche ist auch innerhalb dieses Basissegments eine stärkere Differenzierung vorzunehmen. Insofern scheint für die Warenhäuser eine Doppelstrategie angebracht, die eine Festigung des Basissegments und eine an aktuellen Trends orientierte flexible Zielgruppen-Neuorientierung beinhaltet.

Im Hinblick auf den Globalitätsgrad muß abschließend kritisch vermerkt werden, daß die gesamtunternehmensbezogene Beschreibung der Zielgruppen eine konkrete Umsetzung in segmentspezifische Marketingstrategien relativ schwierig erscheinen läßt. Deshalb müssen die Zielgruppen der Warenhäuser im Rahmen der disaggregierten waren- und filialgruppenbezogenen Planung genauer spezifiziert werden. Erst durch diese Konkretisierung lassen sich die im Sinne der Kundenorientierung erforderlichen Akzentuierungsund Profilierungsziele der Warenhäuser realisieren. 


\section{3 Positionierung von Warenhäusern im Konkurrenzumfeld}

Parallel zur Wahl der Zielgruppenstrategie muß die Warenhausleitung ihre Marktposition durch die Festlegung der strategischen Verhaltensweise gegenüber den Wettbewerbern bestimmen.

Ausgangspunkt dieser Konkurrenzstrategie bildet die $\mathrm{Ab}-$ grenzung der relevanten Wettbewerber. Dabei ist es zweckmäßig, eine möglichst weite Konkurrenzabgrenzung ${ }^{1}$ vorzunehmen, um wichtige Verschiebungen auf der Anbieterseite ebenso wie Veränderungen in den Betriebsformenpräferenzen der Kunden rechtzeitig erkennen und richtig reagieren zu können.

Dementsprechend müssen Warenhäuser neben der unmittelbaren Warenhauskonkurrenz zusätzlich die mittelbaren

Konkurrenten in den übrigen Betriebsformen des institutionellen Einzelhandels berücksichtigen und jeweils spezifische Konkurrenzstrategien entwickeln ${ }^{2}$.

Im oligopolistisch strukturierten Markt der unmittelbaren Warenhauskonkurrenten übernimmt Karstadt die Position des "Marktführers", während Kaufhof und Hertie als "Herausforderer" und schlieBlich Horten als" Marktfolger" zu kenn-

1 Die meisten Autoren plädieren bei strategischen Fragestellungen für eine weite Konkurrenzabgrenzung. Vgl. z.B. Abell, D.F., Hammond, J.S., a.a.O., S. 51 ff.; Meffert, H., Absatzpolitik, a.a.O., S. 104; ähnlich auch: Nieschlag, R., Dichtl.,E., Hörschgen, M., a.a.O., S. 441 .

2 Dabei basieren die strategischen Utberlegungen auf ausführlichen Konkurrenzanalysen. Diese decken tatsächlich vorhandene Wettbewerbsverhältnisse auf und untersuchen sie auf ihre Auswirkungen hinsichtlich des eigenen Warenhauskonzerns. Vgl. hierzu z.B. Dunst, K., Konkurrenzanalyse, in: Marketing Enzyklopädie, Bd. 2, München 1974, S. 147-154, hier S. 147 (im folgenden zitiert als: Dunst, K., Konkurrenzanalyse); Hoffmann, K., Die Konkurrenzuntersuchung als Determinante der langfristigen Absatzplanung, Göttingen 1979, hier S. 10 f.. 
zeichnen sind ${ }^{1}$. Die Bestimmung der Hauptkonkurrenten innerhalb des gesamten industriellen Einzelhandels hat sich an der Entwicklung ${ }^{2}$ und Strategie der übrigen Betriebsformen ${ }^{3}$ zu orientieren. Demnach lassen sich als Hauptkonkurrenten die Verbrauchermärkte (und SB-Warenhäuser) sowie der Facheinzelhandel herausschälen.

Obgleich die konkrete Ausprägung der Konkurrenzstrategie von der jeweiligen Unternehmenssituation und -zielsetzung abhängt ${ }^{4}$, lassen sich generelle Typen relevanter Konkurrenzstrategien für Warenhäuser bestimmen.

1 Die Ermittlung der Marktanteile gestaltet sich insofern schwierig, als jeder Warenhauskonzern in mehr oder weniger starkem Umfang auch in anderen Betriebsformen tätig ist. Beschränkt man sich auf den klassischen Warenhausbereich, so kann man etwa folgende Verteilung zugrunde legen (für 1977): Karstadt: 33 \%, Kaufhof: 25 \% ; Hertie: 268 ; Horten: $16 \%$. Damit liegen Kaufhof und Hertie nahezu gleich auf dem 2. Rangplatz. Weitere kleinere Warenhauskonkurrenten halten lediglich einen Marktanteil von insgesamt ca. $10 \%$ und haben allenfalls regionale Bedeutung, z.B. "Breuninger" (Stuttgart) oder "Schneider" (Freiburg).

$2 \mathrm{Vgl}$. hierzu insbesondere die Ausführungen im Kapitel 1.21 dieser Arbeit.

$3 \mathrm{Vgl}$. neben den Ausführungen im Kapitel 1.12 der Arbeit z.B. Bates, A.D., The Troubled Future of Retailing, in: Business Horizons, Aug. 1976, S. 22-28; Eggert, U., Profilierungschancen für Fachgeschäfte, in: Rationeller Handel 6/1979, S. 40-44; Derselbe, Fachhandels-Strategie für die 8oer Jahre: Die Marketing-Lücke schließen, in: Rationeller Handel, 3/1979, S. 18-23; Derselbe, Handel 80: Die Zeit der Dinosaurier, in: ASW 12/1978, S. 90-96; Goodrich, J.N., Hoffmann, J.A., Warehouse Retailing: The Trend of the Future?, in: Business Horizons, April 1979, S. 45-50; Meffert, H., Perspektiven, a.a.O., S. 70 ff..

$4 \mathrm{Vgl}$. hierzu z.B. die Ausführungen von Jäger, F., Das Konkurrenzverhalten als Problem der Marketingplanung, Diss. Münster 1977, S. 19 ff.. 


\subsection{Typen von Konkurrenzstrategien in Warenhäusern}

Die möglichen Verhaltensweisen eines Warenhauses gegenüber seinen Wettbewerbern lassen sich auf einem Kontinuum abbilden, das sich vereinfachend in die zwei Bereiche offensiv und defensiv aufteilen läßt. Dabei ist offensives Verhalten als aktives, eigenständiges Marketing, defensives Verhalten dagegen als passives, reaktives Orientieren des Marketing am Wettbewerber zu verstehen. Zum anderen können die Auswirkungen der jeweils realisierten Konkurrenzstrategie langfristig in einer völligen Ausschaltung des Wettbewerbs oder in einer Erhaltung des Wettbewerbs mit evtl. anderen Strukturen liegen. Durch die Kombination der beiden Dimensionen "Verhalten" und "Auswirkung" erhält man die in Abbildung 14 dargestellten.konkurrenzbezogenen strategietypen.

\begin{tabular}{|l|l|l|}
\hline $\begin{array}{l}\text { Angestrebte } \\
\text { Auswirkung }\end{array}$ & $\begin{array}{l}\text { Erhaltung des } \\
\text { Wettbewerbs }\end{array}$ & $\begin{array}{l}\text { Ausschaltung } \\
\text { des Wettbewerbs }\end{array}$ \\
\hline defensiv & $\begin{array}{l}\text { Anpassungsstrate- } \\
\text { gie } \\
\text { Rückzugstrategie } \\
\text { Ausweichstrategie }\end{array}$ & $\begin{array}{l}\text { Kooperations- } \\
\text { strategie }\end{array}$ \\
\hline offensiv & $\begin{array}{l}\text { Profilierungs- } \\
\text { strategie }\end{array}$ & Kampfstrategie \\
\hline
\end{tabular}

Abb. 14 : Systemantisierung von Konkurrenzstrategien eines Warenhauses

Verhält sich das Warenhaus defensiv und wird der Wettbewerb langfristig erhalten, so sind Anpassungs-, Rückzugs- und Ausweichstrategien zu diskutieren. Dient das defensive Verhalten der Ausschaltung des Wettbewerbs, so ist die Kooperationsstrategie $\mathrm{zu}$ analysieren. Bei offensivem Verhalten des betrachteten Unternehmens 
sind bei angestrebter Erhaltung des Wettbewerbs Profilierungsstrategien und bei angestrebter Ausschaltung des Wettbewerbs Kampfstrategien zu nennen ${ }^{1}$.

Diese Strategien werden im folgenden isoliert analysiert und auf ihre Eignung als generell für die gesamte Unternehmung gültige Verhaltensweise überprüft ${ }^{2}$.

Verfolgt ein Warenhaus eine Kampfstrategie, so will es seine Konkurrenten ganz oder teilweise vom Markt verdrängen. Die Realisierung dieser Strategie ${ }^{3}$ ist an folgende Voraussetzungen ${ }^{4}$ geknüpft:

- das Warenhaus muß in der Lage sein, die von den Konkurrenten abgezogene Nachfrage zu befriedigen,

- es benötigt starke finanzielle Reserven, um vorübergehend auftretende Verluste abdecken zu können und

- es muß in mehreren Bereichen deutliche Uberlegenheit aufweisen, als Voraussetzung, um genügend große Nachfrage auf sich ziehen zu können.

Aufgrund der oligopolistischen struktur ist derzeit wohl keiner der vier Warenhaus-Konzerne in der Lage, die für eine Kampfstrategie notwendigen Mittel aufzubringen oder die dadurch entstehende Nachfrage alleine $z u$ befriedigen. Ferner ist bei einer Kampfstrategie der Warenhäuser untereinander eine weitere Schwächung der Betriebsform insgesamt zu befürchten, so daß letzten Endes auch der "überlebende" Konzern in Gefahr wäre.

1 Damit wird weitgehend der systematisierung von Hoffmann gefolgt. Vgl. Hoffmann, K., Die Konkurrenzuntersuchung als Determinante der langfristigen Absatzplanung, Göttingen 1979 , S. 47 f..

2 Dabei wird hier der Methodenaspekt in den Hintergrund gerückt. Aufgrund der fehlenden realen Planungssituation wird darauf verzichtet, ein Punktbewertungsmodell zu entwickeln, sondern der qualitativen, mit Tendenzaussagen arbeitenden Beurteilung der Vorzug gegeben.

3 Hier wird nur auf die Anwendung marktkonformer Mittel Bezug genommen.

4 Vgl. hierzu Ilofimann, K., a.a.O., S. 235 f.. 
Diese Gründe gelten in noch stärkerem Maße für die zweite Konkurrenzebene, die konkurrierenden Betriebsformen. Es wäre vermessen, als einzelner Warenhauskonzern eine generelle Kampfstrategie gegenüber dem Fachhandel oder den Verbrauchermärkten einzuschlagen. Eine solche Strategie wäre allenfalls unter der irrealen Voraussetzung einer gemeinsamen Vorgehensweise aller Warenhäuser zu diskutieren; selbst dann ergäben sich jedoch - von rechtlichen Einwendungen abgesehen - aufgrund des insgesamt nicht ausreichenden ökonomischen Potentials erhebliche Bedenken bezüglich der Erfolgsträchtigkeit der strategie.

Mit einer Profilierungsstrategie verfolgt ein Warenhaus das Ziel einer eindeutigen positiven Abgrenzung von der Konkurrenz derart, daß die Konsumenten sich in einer Wahlsituation für die eigene Unternehmung entscheiden ${ }^{1}$. Die Verfolgung dieser Strategie ist an zwei Voraussetzungen gebunden:

- die Möglichkeit eines differenzierten Einsatzes der Marketing-Instrumente muß gegeben sein

- das Warenhaus mu in der Lage sein, in einem (oder mehreren) für die Wahl der Einkaufsstätte maßgeblichen Bereich(en) die Konkurrenz zu übertreffen ${ }^{2}$.

Auf der Ebene der unmittelbaren Warenhauskonkurrenten sind diese beiden Voraussetzungen erfüllt: Jeder Warenhauskonzern realisiert bereits den differenzierten Instrumente-

1 Diese Zielsetzung steht im Grunde hinter dem Einsatz des gesamten Marketinginstrumentariums. Insofern wird in den allgemein $\mathrm{zu}$ verfolgenden zielen der marktorientierten Unternehmenspolitik auch häufig auf profilierungsfördernde Aspekte Bezug genommen. So z.B. durch die Ziele "Erhöhung der Kundenpräferenz", "Positives Image", "Verstärkung der Kaufabsicht". Vgl. hierzu Meffert, H., Marketing, a.a.O., S. 74 .

2 z.B. Sortimentsattraktivität, Auswahl, Image usw.. 
einsatz und in der realen Situation zeigen sich zumindest bei filialgruppen- und warengruppenspezifischer Differenzierung mehr oder weniger deutliche Vorteile für den einen oder anderen Warenhauskonzern. Die Aufgabe der Warenhausleitung besteht nun darin, langfristig wirksame MaBnahmen zum Ausbau der Stärken und zum Abbau der Schwächen zu entwickeln. Dabei werden die Ansatzpunkte je nach Marktstellung anders zu suchen sein. Während der Marktführer sich aus einer Position der relativen Stärke heraus auf die Verteidigung seiner erreichten Stellung durch Absicherung seiner spezifischen stärken konzentrieren kann, sind die Herausforderer gezwungen, ihre schwächen durch besondere Anstrengungen $z u$ beseitigen. Dabei entspricht es dem Grundsatz der Profilierungsstrategie, auf eine"Kopie"des Marktfürers zu verzichten und dem Wettbewerber etwas Gleichwertiges bzw. Besseres entgegenzusetzen.

Ähnlich wie bei den unmittelbaren Konkurrenten ist auch auf der Ebene der Betriebsformenkonkurrenz die Notwendigkeit einer Profilierungsstrategie allgemein erkannt. Auf der Basis einer detaillierten Stärken/SchwächenAnalyse dieser Betriebsformen muß das Warenhaus durch geeignete Strategien die Distanz zwischen den jeweiligen Konkurrenzstärken und den eigenen Schwächen abbauen.

Ein Warenhaus verfolgt eine Anpassungsstrategie, wenn es sich mit seinem Marketinginstrumenteeinsatz an den der Konkurrenten anpaßt und damit deren Marketing-Konzepte imitiert. Auf der Ebene der Warenhauskonkurrenten empfiehlt sich diese Strategie allenfalls für den"Marktfolger", in keinem Fall aber für den"Herausforderer." Er muB dem Marktführer eine qualifizierte, gleichwertige, eigenständig entwickelte Marketingstrategie entgegenstellen, um dessen Marktposition zu erreichen. Dabei können durchaus partielle Anpassungen in ausgewählten Waren- oder Filial- 
gruppen erfolgen. Im Hinblick auf die Betriebsformenkonkurrenz können Warenhausunternehmen eine weitere negative Entwicklung der Marktstellung nur aufhalten bzw. umkehren, wenn sie eigenständige Marketingkonzepte entwickeln, die ihnen in den Augen der potentiellen Käufer einen Vorteil bieten. Auch hier ist die partielle Anpassung durch die Imitation spezifischer Strategieansätze der anderen Betriebsformen bzw. der Versuch, einzelne, die besondere Stärke einer Betriebsform begründende Elemente zu kopieren, möglich.

Ein Warenhaus verfolgt, wenn es bewußt und planmäßig den Abbau seines Marktanteils hinnimmt, die Rückzugsstrategie ${ }^{2}$. ziel dieser strategie ist es, ohne große Investitionen aus der Stellung im bisherigen Absatzmarkt nock soviel Nutzen wie möglich zu ziehen. Als generelle Verhaltensweise wird diese Strategie für kein Warenhaus 1.n. Fraqe kommen. Bei warengruppen- und filialspezifischer Differenzierung erscheint jedoch die Entscheidung, sich aus einem regionalen Markt zurückzuziehen oder bestimmte Sortimentsteile zu eliminieren, durchaus denkbar. In solchen Fällen besteht das strategische Ziel des Warenhauses darin, die freiwerdenden Ressourcen (Rauin, Personal, Finanzmittel) auf Märkten mit besseren Chancen einzusetzen.

Eine Ausweichstrategie bietet sich für ein Warenhaus an, um die direkte Konfrontation ${ }^{3}$ mit den Konkurrenten

1 Hier ist als Beispiel das tiefe Sortiment oder der Trend $\mathrm{zu}$ qualifiziertem Personal in bestimmten Abteilungen des Warenhauses zu nennen; eindeutig Ansatzpunkte, die die Stärke des Fachhandels bestimmen.

2 Es ist auch der Fall denkbar, daß eine Unternehmung durch gravierende Fehler in der Marktbearbeitung gezwungen ist, einen Markt aufzugeben.

3 Dabei tragen diese Strategien teilweise auch gleichzeitig zur Profilierung bei. 
zu vermeiden. In diesem Fall lassen sich drei idealtypische Fälle des ausweichenden Konkurrenzverhaltens unterscheiden, nämlich

- an neuen, d.h. bisher von den unmittelbaren Warenhauskonkurrenten unbesetzten Standorten Filialen zu eröffnen 1

- neue Sortimentsteile aufzunehmen und über das bestehende Filialsystem dem Kunden anzubieten oder

- an neuen Standorten völlig abweichende verkaufsstrategische Konzepte zu realisieren.

Damit wird deutlich, daß diese Ansätze partielle Strategien darstellen, die auf warengruppen- oder filialbezogener Ebene realisiert werden, jedoch nicht die globale Konkurrenzstrategie bilden können.

Auf der Ebene der Betriebsformenkonkurrenten ist die Ausweichstrategie aufgrund der intensiven Konkurrenz im Einzelhandel nahezu unmöglich. Lücken im Sinne von weniger konkurrenzintensiven Bereichen lassen sich nur noch in wenigen Ausnahmefällen finden und werden i.d.R. in relativ kurzer Zeit von Konkurrenten ebenfalls besetzt ${ }^{2}$.

Bei Verfolgung einer Kooperationsstrategie arbeitet ein Warenhaus mit Einzelhandelsunternehmen im Marketingbereich zusammen $^{3}$. Diese Strategie empfiehlt sich, wenn das Wa-

1 Aufgrund der intensiven Standortabdeckung ist diese Möglichkeit heute nur noch im Ausnahmefall gegeben.

2 So ist z.B. der Verkauf von Reisen - ursprünglich ein Diversifizierungsbereich der Warenhäuser - inzwischen zum festen Bestandteil auch anderer Betriebsformen geworden (z.B. Lebensmittelfilialisten).

3 Hier erfolgt eine Einengung auf Kooperation im Marketingbereich. Selbstverständlich sind auch Kooperationen z.B. im Beschaffungs-, Finanz- oder Personalbereich denkbar. Vgl. hierzu die anschaulichen Beispiele bei Bidlingmaier, H., Jacobi, W. Uherek, Wiesbaden 1967, S. 353-395, hier S. 367 ff. (im folgenden zitiert als: Bidlingmaier, J., Kooperation). 
renhaus hierdurch eine - gegenüber individueller Aufgabenerfüllung - als positiv gewertete Variation der Struktur einzelner, mehrerer oder aller Elemente des Zielsystems erwarten kann ${ }^{1}$. Sie kann allerdings nur bei Einhaltung der rechtlichen Vorschriften und bei der Bereitschaft der Kooperationspartner, die Eigenständigkeit partiell aufzugeben, realisiert werden. Damit ist sie im Bereich der Warenhauskonkurrenz als generelle Konkurrenzstrategie auszuschlieBen.

Sie wird dagegen durchaus - wenn auch selten - im Bereich der Betriebsformenkonkurrenz realisiert. Allerdings kann aus den in letzter Zeit zunehmenden Kooperationen in der Warenhauspraxis (z.B. Horten und Edeka) noch keine Tendenzwende im Verhalten der Warenhäuser abgeleitet wer$\operatorname{den}^{2}$.

Aufgrund der vorangegangenen Ausführungen ist die Festlegung einer einzigen Konkurrenzstrategie und ihrer generellen Verbindlichkeit für ein gesamtes Warenhausunternehmen als problematisch anzusehen. Abgesehen von der Profilierungsstrategie sind die übrigen Verhaltensweisen nur in bestimmten Situationen sinnvoll einzusetzen. Damit wird für die Warenhäuser i.d.R. ein mehrdimensionaler konkurrenzstrategischer Ansatz notwendig. Ausgehend von einer Profilierungsstrategie gegenüber den unmittelbaren Warenhauskonkurrenten sowie den übrigen Betriebsformen sind die übrigen Strategieansätze allenfalls partiell, d.h. in der Regel auf der disaggregierten Warengruppenoder Filialgruppenebene zu realisieren.

$1 \mathrm{Vgl}$. Schneider, D., Zur Problematik der Zielbestimmung in Marketing-Kooperationen, in: Modernes Marketing - Moderner Handel, hrsg. v. J. Bidlingmaier, Wiesbaden 1972, S. 89-98, hier S. 95.

2 Allerdings verstärkt sich insbesondere im Bereich der Serviceleistungen der Trend zur Kooperation. Hierfür sprechen z.B. die Kooperationen mit Fotogeschäften, Wäschereien, Schlüsseldiensten usw.. 


\subsection{Analyse der Marktposition von Warenhäusern im Konkurrenzumfeld}

Die mehrdimensionale Konkurrenzstrategie findet ihren Niederschlag in der Positionierung eines Warenhauses gegenüber seinen unmittelbaren und mittelbaren Konkurrenten. Dabei steht die Beurteilung der Leistungsfähigkeit eines Warenhauses im Vergleich zu der der Konkurrenz durch den aktuellen und potentiellen Käufer im Mittelpunkt ${ }^{1}$. Letztlich bestimmt er durch seine Entscheidung über Kauf oder Nichtkauf den Erfolg der Konkurrenzstrategie und damit die Richtigkeit der Positionierungsentscheidung im Konkurrenzumfeld.

Durch die unmittelbare Orientierung der Wettbewerbsstrategie am Käufer wird deutlich, daß sich diese Positionierungsentscheidung eines Warenhauses an den kaufbestimmenden Faktoren orientieren muß, anhand derer eine Betriebsform bzw. eine Einkaufsstätte beurteilt wird und die somit die Determinanten der Einkaufsstättenwahl darstelien ${ }^{2}$.

$\mathrm{Zu}$ diesem Problemkreis wurden im Hinblick auf den Betriebsformenverglelch in den letzten Jahren eine Reihe von Untersuchungen durchgeführt, wobei die folgenden Merkmale aufgrund ihrer zentralen Bedeutung bei der Einkaufsstättenwahl der Konsumenten zur Charakterisierung des Leistungsprogramms herangezogen wurden: Auswahl, Qualität, Preis, Kundendienst, Sonderangebote, Beratung und Einkaufs-

1 Dies zeigt die enge Verzahnung zwischen den einzelnen Komponenten der strategischen Rahmenentscheidung.

$2 \mathrm{Vgl}$. hierzu auch den theoretischen tberblick bei: Heinemann, M., Einkaufsstättenwahl und Firmentreue von Konsumenten, Diss. Münster 1974, S. $22 \mathrm{ff.}$. 
atmosphäre ${ }^{1}$. Danach ergeben sich für die Betriebsformen Warenhaus $^{2}$, Fachgeschäft, Verbrauchermarkt und Versandhandel die in der Abbildung 153 dargestellten Profile.

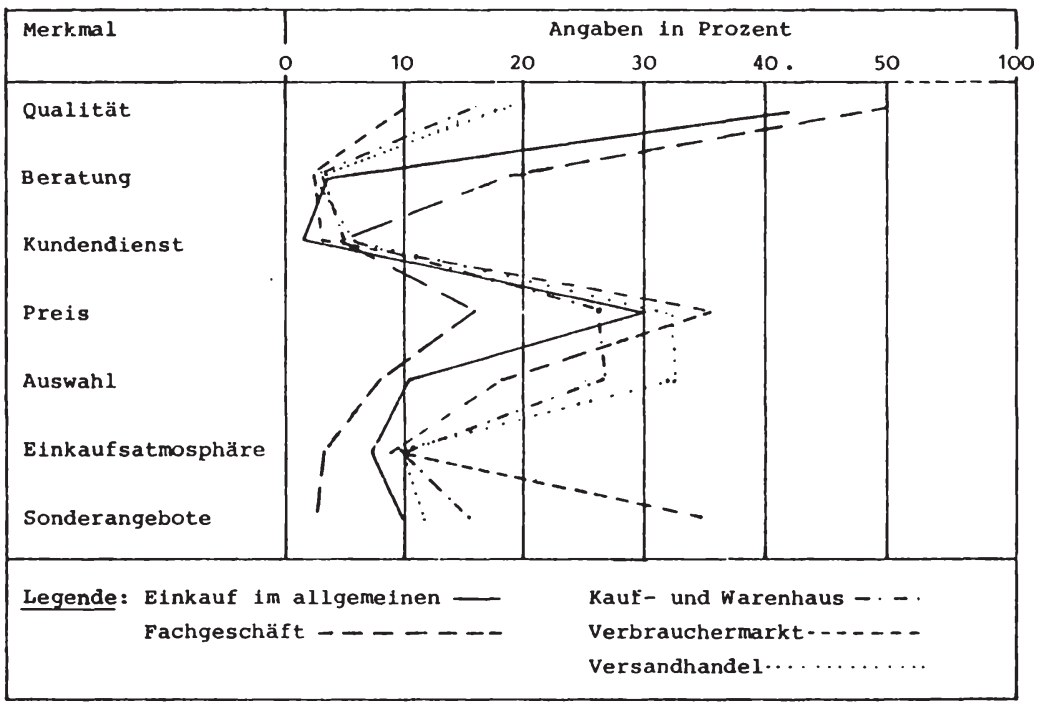

Abb. 15 : Leistungsprofil ausgewählter Betriebsformen

1 Dieser Merkmalskatalog liegt z.B. der BBE-Untersuchung und der Tietz-Studie zugrunde. Vgl. hierzu BBE (Hrsg.), a.a.O., S. $133 \mathrm{ff.;}$ Tietz, B., Einzelhandelsleistung, a.a.0., s. 47 .

2 Tietz untersucht dabei "Kauf- und Warenhäuser".

3 Quelle: nach Tietz, B., Einzelhandelsleistung, a.a.o., S. 47 . 
Aus der Beurteilung der Betriebsformen durch die Konsumenten lassen sich für die Position der (Kauf- und) Warenhäuser ${ }^{1}$ im Markt folgende Feststellungen ableiten:

- die charakteristischen Leistungsprogrammerkmale sind der Preis und die Auswahl

- mit deutlichem Abstand folgen Qualität und Sonderangebote

- mit weiterem Abstand folgt die Beratung

- als relativ unbedeutend werden der Kundendienst und die Einkaufsatmosphäre gesehen.

Diese Ergebnisse stimmen im großen und ganzen auch mit anderen Untersuchungen überein. So wurde in einer Analyse von Anders ${ }^{2}$ die "große Auswahl" als das dominierende Leistungsmerkmal für das Warenhaus ermittelt. Versuche, durch die Hinzunahme weiterer Merkmale ein differenzierteres Bild zu erhalten, erwiesen sich als unterschiedlich erfolgreich. Während die zusätzliche Berücksichtigung des Kriteriums "Lage" (i.S.v. nahe zur Wohnung) für das Warenhaus als unbedeutend gesehen wurde, wiesen die Befragten den Kriterien "freundliche Bedienung" und "übersichtliche Warenanordnung" hohe Priorität $\mathrm{zu}^{3}$.

Ausgehend von derartigen Untersuchungen besteht die Aufgabe der Warenhausleitung darin, ihre Positionierung als Betriebsform so vorzunehmen, daß die in den Augen der aktuellen und potentiellen Käufer relevanten Stärken betont und die Schwächen gemildert werden. Im Hinblick auf die zu entwickelnden Marketingstrategien weisen die Ergebnisse derartiger Analysen den Vorteil auf, daß die

1 Vgl. im einzelnen Tietz, B., Einzelhandelsleistung, a.a.0., S. 47 .

2 Vgl. Anders, H.J., a.a.O., S. 10.

3 Ergebnis der Imageanalyse eines Warenhauskonzerns. 
zugrundegelegten Merkmale unmittelbar in Marketing-Aktivitäten umgesetzt werden können (z.B. Auswahl auf Sortimentsbreite und -tiefe, Beratung auf persönlichen Verkauf bzw. Bedienungssystem usw.). Diese Analysen gewinnen für strategische Entscheidungen im Warenhaus weitere Bedeutung, wenn sie im Hinblick auf die Positionierung gegenüber den unmittelbaren Warenhauskonkurrenten durchgeführt werden. Die Abbildung 16 zeigt dementsprechend das Beurteilungs-Profil von vier Warenhauskonzernen ${ }^{1}$.

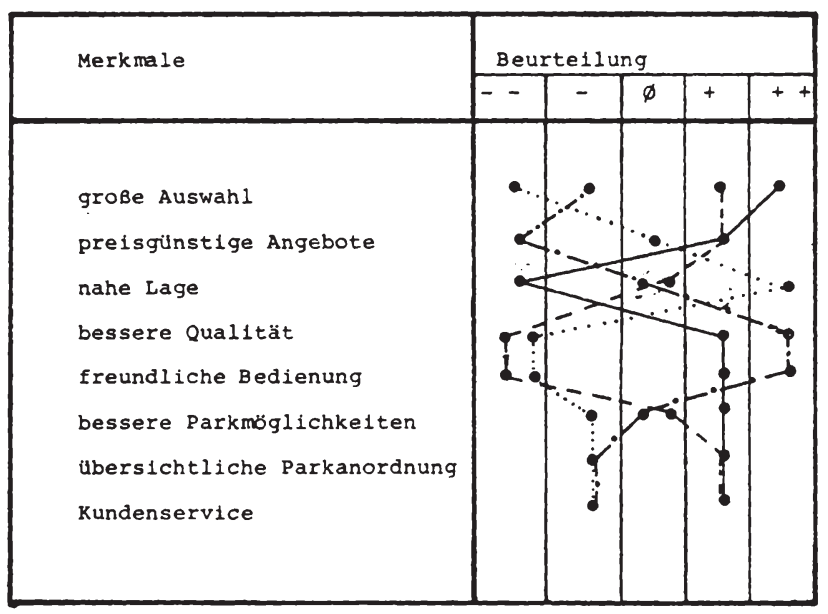

Legende: Warenhaus $\mathrm{A}--$

Warenhaus $\mathrm{B}$
Narenhaus C....

Warenhaus $\mathrm{D}-\cdot-$.

Abb. 16 : Beurteilung von vier Warenhauskonzernen anhand ausgewählter Merkmale 1 Die Profile beruhen auf Plausibilitätsüberlegungen des
Verfassers. 
Aus dem Blickwinkel des Warenhauses B ergeben sich z.B. eindeutige Stärken im Hinblick auf die große Auswahl und die preisgünstigen Angebote und damit in jenen Bereichen, die allgemein für die Betriebsform Warenhaus größte Relevanz besitzen. Hier gilt es unbedingt, die erzielte Konkurrenz-Positionierung in der Kundeneinschätzung zu halten. Für die Warenhäuser $C$ und $D$ muß es dagegen ein zentrales $\mathrm{ziel}$ sein, langfristig die negative bzw. durchschnittliche Beurteilung in diesen Dimensionen $z u$ verbessern. Ihre jetzige Positionierung im Markt gegenüber den anderen Warenhäusern erscheint für den langfristigen Erfolg - insbesondere vor dem Hintergrund des zunehmenden Konkurrenzdrucks anderer Betriebsformen - gefährdet.

Diese relativ globale Operationalisierung der Positionierung von Warenhäusern im Konkurrenzumfeld muß je nach Entscheidungsfeld auf disaggregierterer Ebene fortgeführt werden. Dem dualen Konzept entsprechend ist dabei insbesondere die Positionierung von Warengruppen und Filialgruppen von Bedeutung. Für diese müssen ebenso wie auf der Gesamtunternehmensebene Beurteilungsprofile im Konkurrenzumfeld ermittelt werden. Hierdurch lassen sich einerseits Unterschiede zur durchschnittsorientierten Gesamt-Positionierung ermitteln und andererseits spezifische, SGEbezogene Rahmenentscheidungen treffen, die dann in einem kundenspezifisch differenzierten MarketinginstrumenteEinsatz ihren Niederschlag finden. 1

1 Die Warenhauskonzerne führen sog. Standortanalysen durch, deren wesentlicher Bestandteil die Bestimmung der Ist-Positionierung der Warengruppen $b z w$. Abteilungen einer einzelnen Filiale ist. Eine grundlegende Darstellung der meßtheoretischen Probleme und Ansätze zu ihrer Lösung findet sich bei Heemeyer, H., a.a.O., S. $61 \mathrm{ff..}$ Vgl. hierzu auch Diller, H., Image-Analyse ohne Spekulation, in: Rationeller Handel, 2/1978, S. 6-14. 
Den aufgezeigten Ansatzpunkten zur Positionierung von Warenhäusern gegenüber den mittelbaren und unmittelbaren Konkurrenten kommt in der strategischen Marketingplanung eine zentrale richtungsweisende Funktion zu. Durch die Rahmenentscheidungen im Hinblick auf die Positionierung im Konkurrenzumfeld werden in Verbindung mit der Kundengruppenstrategie die Freiheitsgrade bei der Entwicklung von Marketing-Basis- und Instrumentalstrategien wesentlich eingeengt. Dies impliziert gleichzeitig, daß jede Beurteilung von Marketingstrategien, die sich aus den Ergebnissen der nachfolgenden Portfolio-Analyse ableiten lassen, stets nur vor dem Hintergrund der getroffenen Rahmenentscheidungen vorgenommen werden darf. 
4. Entwicklung von Marketing-Basisstrategien auf der Grundlage der Portfolio-Methode

Auf der Basis des durch die Ziel- und Positionierungsentscheidung gesteckten Planungsrahmens fällt den Marketing-Basisstrategien die Aufgabe $z u$, die strategischen Stoßrichtungen für die strategischen Geschäftseinheiten in Warenhäusern aufzuzeigen. Da die Marketing-Basisstrategien ihrerseits den Entscheidungsspielraum für die disaggregierte Planung innerhalb der Waren- und Filialgruppen einengen, nehmen sie eine zentrale Koordinations-Funktion bei der Ausrichtung aller Marketingstrategien zu einem in sich geschlossenen, widerspruchsfreien system wahr. Die zweckadäquate Erfüllung dieser Funktion gewährleistet in besonderem Maße die Portfolio-Methode, die durch ihren Ziel-, Käufersegment- und Konkurrenzbezug einerseits sowie durch ihre Konkretisierungshinweise für die Marketing-Instrumentalstrategien die notwendige "Verzahnung" $z u$ den vor-und nachgelagerten Planungsstufen schafft.

\subsection{Ermittlung von Portfolio-Varianten für Warenhäuser auf der Basis der Rahmenentscheidungen}

Folgt man der Grundstruktur der Portfolio-Methode ${ }^{1}$, so stellt sich bei ihrer Anwendung unmittelbar die Frage nach den zugrundeliegenden Schlüsselfaktoren ${ }^{2}$, die die Achsenbezeichnungen und damit den materiellen Gehalt der Portfolio-Matrix bestimmen. Als Schlüsselfaktoren sind für die

1 Vgl. hierzu Kap. 1.3 der Arbeit.

2 Dabei leisten die bislang in der Theorie bzw. praktischen Anwendung vorgeschlagenen Lösungsansätze nur bedingt einen Beitrag, da sie primär auf herstellerspezifische Problemstrukturen ausgerichtet sind. 
Warenhausplanung jene Größen anzuwenden, die die Entscheidungsfindung im Rahmen der strategischen Marketingplanung primär determinieren. Dementsprechend sind sie einerseits aus der aktuellen und zukünftigen MarketingSituation ${ }^{1}$ abzuleiten. Andererseits müssen die relevanten Größen der Zielsetzung der Warenhäuser sowie die segment- und Konkurrenzstrategien bei der Wahl der Schlüsselfaktoren ihren Niederschlag finden.

Unter der Perspektive der Zielorientierung der Schlüsselfaktoren ist zunächst der Deckungsbeitrag als gewinnorientiertes Oberziel bedeutsam. Als quantifizierte Kennzahl kann das Deckungsbeitragsziel zum einen auf Warengruppen in Form des Deckungsbeitrags pro qm bezogen werden. Zum anderen läßt er sich auch filialgruppenspezifisch erfassen, und zwar als Summe der Einzelfilial-Deckungsbeiträge ergibt er den Deckungsbeitrag der gesamten Filialgruppe.

Kommt im Deckungsbeitrag, der mit einer Warengruppe bzw. Filialgruppe erzielt wird, die Konkurrenzzielsetzung nur implizit zum Ausdruck, so stellt der Marktanteil eine Zielgröße dar, in der die Stellung eines Warenhauses am Markt explizit erfaßt wird. Insbesondere auf stagnierenden bzw. nur leicht wachsenden Märkten mit starkem Konkurrenzdruck wie dem Einzelhandel kommen dem Marktanteil wesentliche Erklärungs- und Implikationsfunktionen für strategische Entscheidungen $\mathrm{zu}$.

Im Hinblick auf den notwendigen Kundensegmentbezug und die kundenorientierten zielsetzungen ist weiterhin

1 Hierbei rücken in jüngster zeit insbesondere auch rechtliche Aspekte als Nebenbedingungen in den Vordergrund. Vgl. hierzu z.B. Ahlert, D. ,Pollmüller, D., Die Rechtsordnung als institutioneller Rahmen des Marketing, in: Handbuch Marketing, Bd. 1, Hrsg. Koinike, J., Gernsbach 1978, S. $117 \mathrm{ff.;} \mathrm{vgl.} \mathrm{auch} \mathrm{die} \mathrm{ausführlichen}$ Checklisten bei Lessing, R., Welke, W., Marketing, Bd. 2 der Schriftenreihe Strategische Unternehmensfürung, Hrsg. G. Kienbaum, München 1976 , S. $162 \mathrm{ff.}$

2 Z.B. aufgrund seiner Beziehung zum Erfahrungskurveneffekt (siehe Kapitel 1.3 der Arbeit). 
das Image als relevanter Schlüsselfaktor zu nennen. Der langfristige Erfolg eines Warenhauses hängt in starkem Maße davon $a b$, inwiefern es gelingt, durch eine adäquate Akzentuierung und Profilierung Kunden an die eigene Einkaufsstätte zu binden. Dabei ist dem dualen Konzept folgend das Image nach Warengruppen und Filialgruppen differenziert zu ermitteln. Für die Planung der Basisstrategien erscheint es zunächst ausreichend, das Image in den wichtigsten einkaufsbestimmenden Dimensionen "Auswahl", "Preis", "Bedienung/Beratung" und "Qualität" zu erfassen". Da sich das Image primär durch die Erfahrung mit einer Einkaufsstätte am spezifischen Standort bildet, muß eine mehrfache Aggregation stattfinden, die zum einen über die regionale Warengruppenbeurteilung, zur Gesamtbeurteilung einer Warengruppe gelangt und zum anderen über das regionale Abteilungsimage das Filial- und schlieBlich das Filialgruppenimage erfaßt. Dabei ist es aus Gründen der praktischen Handhabbarkeit notwendig, die Einzeldimensionen des Warenhausimage $\mathrm{zu}$ einem einzigen Imagewert $\mathrm{zu}$ verdichten. In dieser Interpretation schafft das Image im Rahmen der Portfolio-Planung sowohl den Bezug zur Zielgruppe als auch zur Konkurrenz ${ }^{2}$.

Im Hinblick auf die geforderte Marktorientierung der Schlüsselfaktoren ist das Marktwachstum aufgrund seiner Funktion als zentraler Indikator für die zukünftigen Chancen einer Warengruppe oder Filialgruppe bedeutsam ${ }^{3}$. Die wachstumsorientierte Formulierung der quantitativen Marketingziele verlangt, daß die Warenhausleitung ihre ver-

1 Weitere Imagedimensionen sind primär im Rahmen der disaggregierten Planung von Interesse.

2 Oftmals wird bei Imageanalysen die Beurteilung der Konkurrenz gleichzeitig mit erhoben. Hierdurch erhält sie dann den Charakter einer konkurrenzbezogenen StärkenSchwächen-Analyse.

3 Die zentrale Bedeutung der Wachstumschancen sowie der hierfür relevanten Subfaktoren betont z.B. auch $\mathrm{Hill}$, W., Umweltanalyse und Unternehmensplanung, in: Die Unternehmung 1977 , S. 289-305, hier S. 300. 
stärkte Aufmerksamkeit auf Bereiche lenkt, die auch in Zukunft Chancen bieten. Dies betrifft sowohl die verschiedenen Warengruppen, die völlig unterschiedliche Wachstumsraten aufweisen ( $z$.B. weist der gesamte Freizeitbereich starke Zuwachsraten auf, der Lederwarenbereich dagegen stagniert weitgehend), als auch die einzelnen Filialgruppen, die z.B. aufgrund nicht beeinflußbarer Wanderungsbewegungen sowie aufgrund von Veränderungen im Einkaufsverhalten der Konsumenten unterschiedliche Marktwachstumsraten aufweisen können. Um $z u$ verhindern, daß in stark wachsenden Märkten relativ hohe eigene zuwachsraten über eine stetige Verschlechterung des Marktanteils hinwegtäuschen, ist neben dem Marktwachstum das Unternehmenswachstum, ausgedrückt als durchschnittliche Umsatzsteigerung je Warengruppe bzw. Filialgruppe über mehrere Jahre hinweg, bei der Portfolio-Planung zu berücksichtigen.

Das Marktwachstum hängt eng mit dem Konzept des (Produkt-) Lebenszyklus zusammen. Je nach erreichtem Stadium im Lebenszyklus wird das Umsatzwachstum variieren und müssen spezifische Strategieimplikationen abgeleitet werden. Die Betrachtung dieser relevanten Dimension für die Portfolio-Planung in Warenhäusern erfolgt unter der Prämisse, daß die Warengruppen eines Warenhauses entsprechend ihrer marktlichen Entwicklung in der Vergangenheit sowie dem prognostizierten Trend in das (idealtypische) Lebenszyklusmodell zu positionieren sind. ${ }^{1}$ Für Filialgruppen muß diese Einschätzung in Analogie zum Lebenszyklus von Betriebsformen ${ }^{2}$ erfolgen.

1 Dabei muß man sich stets der Problematik des Lebenszykluskonzeptes an sich sowie der Schwierigkeiten, Warenoder Filialgruppen in die einzelnen Phasen einzuordnen, vor Augen halten.

2 Vgl. hierzu die überlegungen auf Seite 31 der Arbeit. 
Die in ihrer Bedeutung skizzierten internen und externen Größen bilden nun die Dimensionen der Portfolio-Matrix. Dabei sind nach der Art ihrer Verknüpfung zwei unterschiedliche Vorgehensweisen denkbar.

Zum einen können jeweils zwei der Dimensionen isoliert berücksichtigt werden. Dahinter steht die Utberlegung, daß die jeweiligen Dimensionen für sich betrachtet wesentliche Strategieimplikationen für Waren- und Filialgruppen zulassen. Insofern ist es sinnvoll, ihren Einfluß auf die Waren- und Filialgruppen isoliert zu betrachten und erst im nachhinein sukzessive durch eine vergleichende Betrachtung bzw. Kombination der Einzelergebnisse die insgesamt zu realisierenden Strategien festzulegen. Diese als mehrstufig-analytisch bezeichnete Vorgehensweise ermöglicht es somit, verschiedene analytische Portfolios mit eindeutig definierten Dimensionen $\mathrm{zu}$ erstellen und jeweils Marketing-Basisstrategien zu entwickeln, die in den nachfolgenden Schritten entweder bestätigt werden oder zu modifizieren sind. Gemäß dieser Vorgehensweise sind die folgenden analytischen Portfolio-Varianten ${ }^{1}$ sukzessive zu diskutieren:

- das Image-Ertragskraft-Portfolio

- das Marktanteils-Ertragskraft-Portfolio

- das Marktanteils-Marktwachstums-Portfolio sowie

- das Marktwachstums-Unternehmens-Portfolio.

Zum anderen können diese bislang isoliert betrachteten Dimensionen - eventuell um weiter unternehmensspezifisch als relevant erachtete Dimensionen ergänzt - mittels der

1 Diese Portfolio-Varianten sind z.T. bereits erfolgreich bei Hersteller- und Handelsunternehmungen eingesetzt worden. 
Punktbewertungsmethode ${ }^{1}$ zusammengefaßt werden. Die verschiedenen Einflußfaktoren sind dabei je nach ihrer unternehmensspezifischen Bedeutung durch Gewichtung in eine Rangordnung zu bringen.

Die Gesamtbeurteilung einer strategischen Geschäftseinheit erfolgt sodann für jede Matrixdimension durch eine additive Verknüpfung der Einzelbeurteilungen. Bei dieser synthetischen Vorgehensweise werden als Matrixdimensionen normalerweise die Marktattraktivität und relative Wettbewerbsstärke bezeichnet. Sie bringen mit den dahinterstehenden Einflußfaktoren die Bedeutung der Markt-bzw. Kundensituation sowie der Konkurrenzlage für die strategische Marketingplanung zum Ausdruck. Dabei sind für die Anwendung der synthetischen Portfolio-Varianten in Warenhäusern diese Dimensionen materiell den warenhausspezifischen Belangen anzupassen. Dies fordert die warenhausspezifische Auswahl und Gewichtung der markt-, kunden- und konkurrenzorientierten Einflußfaktoren. Unter diesem Blickwinkel sind als synthetische Portfolio-Varianten für Warenhauser

- das Marktattraktivitäts-Wettbewerbsvorteile-Portfolio sowie

- das Lebenszyklus-Wettbewerbsstärke-Portfolio

zu diskutieren ${ }^{2}$.

1 Vgl. zur Darstellung und Kritik dieser Methode z.B. Schmitt-Grohé, J., Produktinnovation. Verfahren und Organisation der Neuproduktplanung, Wiesbaden 1972, S. $84 \mathrm{ff.}$.

2 Auch diese beiden Portfolio-Varianten wurden bereits bei praktischen Anwendungen, insbesondere bei Herstellerunternehmen erfolgreich eingesetzt. 
Für die nachfolgende Diskussion der analytischen und synthetischen Portiolio-Varianten wird jeweils auf die sog. Ist-Portfolio-Matrix Bezug genommen. Zu ihrer Darstellung wird im folgenden auf ein empirisches Beispiel zurückgegriffen. Dabei steht die Entwicklung von Warengruppenstrategien'im Vordergrund, wobei für jede PortfolioVariante zusätzlich die sich aus Filialgruppensicht ergebenden Besonderheiten aufgezeigt werden. Die Positionierung der strategischen Geschäfseinheiten in den portfolios basiert auf den zum Betrachtungszeitpunkt abgegebenen Beurteilungen der Warenhausleitung. Auf der Grundlage dieser empirisch ermittelten Portfolios werden im folgenden die wesentlichen Erkenntnisse für die Gestaltung der Marketing-Basisstrategien abgeleitet und interpretiert.

\subsection{Anwendung analytischer Portfolio-Varianten in Waren- häusern}

\subsection{Warengruppen- und Filialgruppenstrategien auf der Basis des Image-Ertragskraft-Portfolios}

Mit dem Image-Ertragskraft-Portfolio werden die angesichts der Warenhauskrise vorrangigen Ansatzpunkte der strategischen Marketingplanung, nämlich einerseits die Kundenurd andererseits die Ertragsorientierung in Beziehung gesetzt. Im Hinblick auf den Kundenbezug verkörpert das Image der Warengruppen bzw. Filialgruppen die unternehmensextern orientierte Größe ${ }^{2}$. Ihr wird als unternehmensinterne, er-

1 Dabei werden jene 10 Warengruppen eines Warenhauskonzerns (im folgenden "Warenhaus $X$ " genannt) zugrunde gelegt, die in der Tabelle 3 aufgeführt sind.

2 Hierzu ist auf die im Rahmen der Imageanalyse ermittelten Werte zurückzugreifen. Um der Gefahr von Fehlinterpretationen vorzubeugen, ist zu überlegen, den aggregierten Imagewert als "Beliebtheits-" Index zu bezeichnen. 
tragsorientierte (Ziel-) Größe der Deckungsbeitrag pro qm ${ }^{1}$ gegenübergestellt. Dies führt $\mathrm{zu}$ der in Abbildung 17 dargestellten Portfolio-Matrix, aus der sich Ansatzpunkte für die Marketing-Basisstrategien von Warengruppen ableiten lassen ${ }^{2}$.

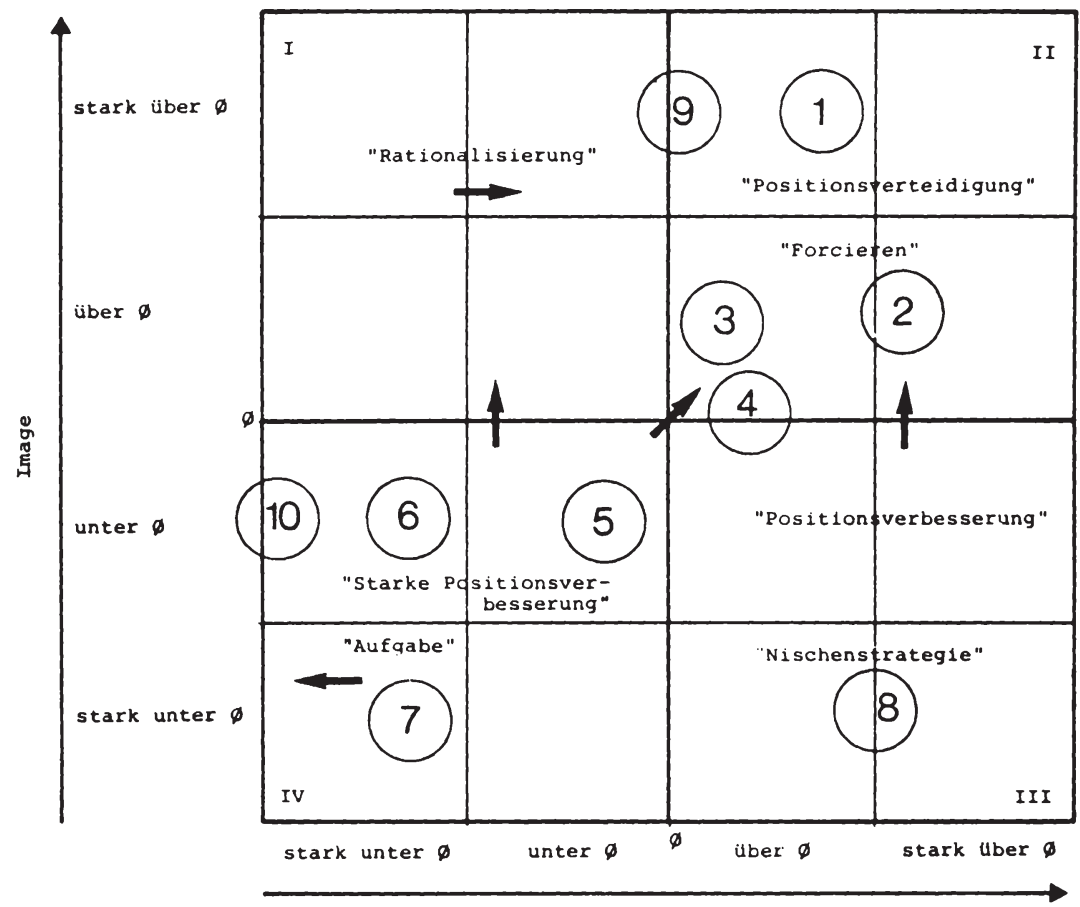

Ertragskraft ( DB/qm )

Abb. 17 : Image-Ertragskraft-Portfolio der Warengruppen des Warenhauses $\mathrm{X}$

1 In diesem Beispiel erfolgt eine Durchschnittsbildung, die die absoluten Deckungsbeiträge in eine qualitative Skala überträgt. Dies dient primär der deutlicheren und einfacheren Unterscheidung der strategischen Felder; selbstverständlich können auch die absoluten werte eingesetzt werden. Für den Fall, daß in einem Handelsbetrieb nur Umsatzzahlen vorliegen, kann auch die Kennziffer Umsatz/qm verwendet werden. Vgl. hierzu auch den Vorschlag von Unkelbach, W., Wiegmann, V.T., Sortimentssieb für Warenhäuser, in: ASW 7/1978, S. 61-63.

2 Barth rechnet dieses Portfolio der kurzfristigen Absatzplanung $z u$ und verwendet es zur Ableitung von flankierenden Maßnahmen. Vgl. Barth, K., Unternehmensführung, a.a. O., S. $199 \mathrm{f}$.. Dies erscheint nicht sinnvoll, da das Image kurzfristig nicht vi: iierbar ist. 
Im Feld I sind Warengruppen positioniert, die durch ein positives Image bzw. eine große Beliebtheit, aber durch eine unterdurchschnittliche Ertragskraft gekennzeichnet sind. Im vorliegenden Beispiel ist allenfalls die Warengruppe Lebensmittel(9) diesem Bereich zuzuordnen. Für diesen Bereich bieten sich Basisstrategien an, die primär innengerichtet auf eine Verbesserung des Deckungsbeitrages wirken. Hierzu zählen z.B. Rationalisierungsstrategien (Kosteneinsparungen, bessere Warenbewirtschaftung) und Veränderungen der Kalkulation, wobei darauf $z u$ achten ist, daB der relativ hohe Beliebtheitsgrad - der ja im Grunde einen Wettbewerbsvorsprung darstellt - nicht beeinträchtigt wird. Dies gilt in der realen Situation der Warenhäuser insbesondere für den Lebensmittelbereich, der in den vergangenen Jahren das Gesamtimage der Warenhäuser allgemein sehr positiv beeinflußt hat ${ }^{1}$.

Die Warengruppen des Feldes II weisen sowohl ein überdurchschnittlich positives Image als auch einen überdurchschnittlich hohen Deckungsbeitrag pro qm auf. Im Beispiel des Warenhauses $x$ sind in diesem Feld die Warengruppen Damenbekleidung (1), Herrenbekleidung (2), Alles für's Kind (3) sowie Schuhe und Kleintextilien (4) positioniert. Für diese Warengruppen gilt es, den erreichten Vorsprung gegenüber den Wettbewerbern sowie den daraus resultierenden positiven Ertragseffekt auf jeden Fall zu halten bzw. $z u$ stärken. Verfügen diese Warengruppen über ein noch nicht ausgeschöpftes Marktpotential, so ist hier eine Forderung zur weiteren Verbesserung der Wettbewerbsvorteile und zur langfristigen Sicherung der Ertragskraft notwendig. Dies

1 Insofern ist es interessant, die zukünftigen Auswirkungen der Horten-Entscheidung, den Lebensmittelbereich auszugliedern, auf das Gesamtimage zu verfolgen. 
trifft im realen Fall bedingt für die Warengruppe Alles für's Kind (3) zu, die vom Marktpotential her noch überdurchschnittliche Zukunftschancen für Warenhäuser eröffnet. Handelt es sich dagegen um Warengruppen, die einer fortgeschrittenen Markt-Lebenszyklusphase zuzurechnen sind, so müssen die positionsverteidigenden Aktivitäten mit möglichst geringen Investitionen erreicht werden, da diese Warengruppen im wesentlichen jene finanziellen Mittel freisetzen sollen, die zum Aufbau starker Imagepositionen bei anderen Warengruppen benötigt werden. So sind z.B. im Bereich der Damen- und Herrenbekleidung insbesondere sortimentsprofilierende überlegungen (z.B. Erhöhung des Markenwarenanteils, Abrundung um obere Qualitätslagen) empfehlenswert, die jedoch stets unter der Prämisse der Erhaltung der Ertragskraft zu beurteilen sind.

Im Feld III sind jene Warengruppen zu finden, die zwar überdurchschnittliche Ertragskraft, aber unterdurchschnittliche Beliebtheit aufweisen, wie z.B. die Warengruppe verschiedener Bedarf (8). Hier greifen alle Basisstrategien Platz, die die Beliebtheit der betreffenden warengruppen beim Konsumenten $z u$ fördern vermögen, wobei dem profilierenden Einsatz der Marketing-Instrumente primäre Bedeutung zukommt (attraktives Sortiment, hohe Qualität, intensive Kommunikation usw.). Die Praxis zeigt, daß in diesem Feld oftmals neu in das sortiment aufgenommene Waren- (oder Artikel-) gruppen positioniert sind, wie z.B. der in Warenhäusern in den letzten Jahren sehr forcierte Sport- und Freizeitbereich. Diese Warengruppen verfügen dann oftmals nur über ein relativ geringes Umsatzvolumen, weisen dafür aber überdurchschnittliche Wachstumsraten auf. Denn mit der Möglichkeit, diese Warengruppen auch im Warenhaus kaufen zu können, muß der Konsument erst vertraut gemacht werden, nur dann kann der "Außen- 
seiter" richtig in das Sortiment hineinwachsen.

Das Feld IV weist unterdurchschnittliche Beliebtheit und unterdurchschnittliche Ertragskraft aus. Im Beispiel sind hier die Warengruppen Textiler Einrichtungsbedarf (5), übriger Einrichtungsbedarf (6), Einrichtungszubehör (7) sowie Restaurant/Café (10) positioniert. Diese Warengruppen stellen echte Problemfälle dar, da sie sowohl im Hinblick auf das Image- bzw. das Ertragsziel negative Auswirkungen zeigen. Insofern legt das Image-Ertragskraft-Portfolio als Basisstrategien entweder eine starke Positionsverbesserung oder eine stufenweise Aufgabe der betroffenen Warengruppen nahe. Die Entscheidung für eine dieser Basisstrategien ist vor der endgültigen Festlegung durch weitere Analysen zu fundieren ${ }^{1}$.

Handelt es sich um Warengruppen in einem Anfangsstadium des Lebenszyklus, die also noch über ein relativ großes Umsatzpotential verfügen, sind starke Anstrengungen zur Verbesserung der Position beim Konsumenten - zunächst unter Mißachtung einer Verbesserung der Ertragskraft sinnvoll. Ist ein zufriedenstellendes $M a B$ an Beliebtheit erreicht, können wleder tjberlegungen platzgreifen, wie sie für das Feld I vorgeschlagen wurden.

Für den Fall, daß der Markt aber weitgehend ausgeschöpft ist, sind Anstrengungen zur Positionsverbesserung nur in Verbindung mit geeigneten Strategien zur Ertragsverbesserung sinnvoll. So wäre z.B. für den Restaurant-Café-Bereich(10) eine konsequente Rationalisierung mittels der Umstellung auf Selbstbedienung durch das Angebot standar-

1 Hier ist insbesondere die Frage des Sortimentsverbundes zu klären. Vgl. hierzu z.B. Böcker, F., Merkle, E., Die Analyse des Sortimentsverbundes, in: Böcker, F., Dichtl, E. (Hrsg.), Erfolgskontrolle im Marketing, Berlin 1975, S. 179-191; Stahl, P., Verbundwirkungen im Sortiment, Diss. Münster 1977. 
disierter Gerichte ${ }^{1}$ evtl. in Verbindung mit einer Reduktion der Verkaufsfläche angezeigt. Ist eine Ertragsverbesserung langfristig nicht zu erreichen, ist zu prüfen, ob die diesen Warengruppen zur Verfügung gestellten Leistungsfaktoren (Verkaufsfläche, Personal, Werbung usw.) nicht eine ertragreichere Verwendung finden können. Für das Beispiel des Restaurant/Café-Bereiches sind unter diesem Aspekt Strategiealternativen denkbar, die von einer stufenweisen Aufgabe der Abteilung bis hin zu einer Ausgliederung durch Vermietung an externe Restaurant-Unternehmen bzw. an örtliche pächter reichen.

Wird das Image-Ertragskraft-Portfolio für Filialgruppen erstellt, so kann die Warenhausleitung hiermit die Imagewerte aller Filialgruppen sowie die Filialgruppen-Dekkungsbeiträge im internen Vergleich analysieren.

Zu den Filialgruppen im Feld I mit relativ hohem Image bei ihren zielgruppen, aber gleichzeitig auch schlechtem Filialgruppen-Deckungsbeitrag zählen jene, die aufgrund ihrer regionalen Lage Warenbewirtschaftsprobleme aufweisen, personalintensiv arbeiten, ungünstige bauliche Voraussetzungen haben oder in ertragsstarken Sortimentsbereichen zu geringe Verkaufsflächenanteile aufweisen. Infolgedessen greifen hier primär Rationalisierungsstrategien Platz, die auf eine Beseitigung der kostensteigenden und ertragsmindernden Faktoren gerichtet sind.

Die Filialgruppen in Feld II müssen dagegen mit ihrem hohen Image und ihrer hohen Ertragskraft in ihrer Position gehalten werden. Hierzu müssen Veränderungen in der wettbewerbsstruktur (z.B. neue Verbrauchermärkte oder Fachgeschäfte) oder aber in der Marktbearbeitungsstrategie der Konkurrenten (z.B. starker Ausbau bestimmter Abteilungen, Neueröffnungen der Warenhauskonkurrenten, Spezial-

1 Z.B. nach dem McDonald-Konzept. 
häuser-Gründung) möglichst frühzeitig erkannt werden, damit der relative Vorsprung gegenüber den Konkurrenten durch geeigneten Marketing-Instrumenteeinsatz gesichert werden kann.

Den Filialgruppen im Feld III, die ein unterdurchschnittliches Image und eine überdurchschnittliche Ertragskraft aufweisen, sind in der Realität primär jene zuzurechnen, die aufgrund einer ungünstigen Lage (z.B. Randlage) oder einer zu geringen Verkaufsfläche ein im Umfang kleineres Sortiment führen müssen. Läßt es die Konkurrenzsituation $z u$, ist eine Nischenstrategie angezeigt, die durch sortimentsprofilierende Aktivitäten einerseits und durch ertragssichernde Rationalisierungsmaßnahmen (z.B. Logistik, Verkaufspersonaleinsatz) andererseits die Position erhält. Kommt als situativer Faktor aber ein starker Konkurrenzdruck hinzu, so sind diese Filialgruppen i.d.R. dem Feld IV, das durch niedriges Image und geringe Ertragskraft gekennzeichnet ist, zuzurechnen. Hier ist im Einzelfall $z u$ überprüfen, ob eine Ertragsverbesserung durch eine Nischenstrategie realisiert werden kann, oder ob durch starke Investitionen (z.B. Umbau, Sortimentsumschichtung, aktive Kommunikation) eine deutliche Positionsverbesserung möglich ist. Werden diesen Aktivitäten geringe Chancen eingeräumt, ist eine stufenweise Desinvestition angezeigt, die über eine abteilungsweise Aufgabe bis hin zur Schließung ganzer Filialen führt. Allerdings sind in diesem Feld i.d.R. auch neueröffnete Filialen zu finden. Denn aufgrund ihrer hohen Anfangsinvestitionen sind ihre wirtschaftlichen Deckungsbeiträge in den ersten Jahren meist sehr gering. Ebenso kann sich ihr Image erst im Verlauf eines längeren Zeitraumes bilden, da es unmittelbar mit der persönlichen Erfahrung der Käufer verbunden ist. Insofern sind für diese Filialgruppen Basisstrategien angezeigt, die auf eine starke Positionsverbesserung 
hinarbeiten und sowohl imagefördernde Aktivitäten (z.B. Bekanntheitsgrad, Demonstration der Leistungsfähigkeit) als auch Rationalisierungsmaßnahmen (z.B. Beseitigung von Anfangsschwierigkeiten, notwendige Umstrukturierungen) umfassen.

Insgesamt betrachtet verdeutlicht das Image-Ertragskraft-Portfolio das Gesamtgefüge der Waren- bzw. Filialgruppen und ermöglicht hierdurch die Entwicklung von Marketing-Basisstrategien aus der strategischen Gesamtsicht des Warenhauses. Darüber hinaus sind die abzuleitenden Strategien auf die zentralen zieldimensionen ausgerichtet und leisten von daher bei ihrer Realisierung einen wesentlichen Beitrag zur Ubberwindung der aktuellen Warenhauskrise. Allerdings ist die Operationalisierung der Imagedimension als problematisch anzusehen. Zum einen führt die Verdichtung der einzelnen Imagewerte $z u$ einem Informationsverlust, da sich die reurteilungsstärken und -schwächen der einzelnen Dimensionen ausgleichen können und die Tendenz zu einer durchschnittlichen Beurteilung fördern. Gleichzeitig verhindert die Verknüpfung das Erkennen von konkreten Strategiehinweisen, die durch eine detaillierte, isolierte Analyse der Beurteilungen zu erhalten sind. Zum anderen ist die vergleichende Betrachtung über mehrere Jahre hinweg bei streng theoretischem Maßstab problematisch, da sich als Durchschnittswert bei jährlich durchgefuhrte Analysen bereits durch die Verschiebung der Einzelbewertungen jeweils ein anderer Wert ergibt ${ }^{1}$. Weiterhin geben die entwickelten strategien ohne Hinzunahme weiterer Informationen z.B. aus anderen Portfolio-Varianten oder weiteren Analysen nur recht globale Verhaltensrichtungen an. So kann insbesondere die Ver-

1 Allerdings halten sich die Verschiebungen des Durchschnittswertes in der praktischen Anwendung in relativ engen Grenzen, so daß zumindest näherungsweise statischkomparative Vergleiche durchzuführen sind. 
nachlässigung des Konkurrenzaspektes zu einer in der realen Situation nicht gerechtfertigten strategieempfehlung führen.

Im Hinblick auf diese Kritikpunkte ist es sinnvoll, das Marktanteils-Ertragskraft-Portfolio als Ergänzungs- und Kontroll-Portfolio hinzuzuziehen.

\subsection{Warengruppen- und Filialgruppenstrategien auf der Basis des Marktanteils-Ertragskraft-Portfolios}

Der Ersatz der am Konsumenten orientierten Zielgröße "Image" durch die am Konkurrenten orientierte Zielgröße "Marktanteil" zeigt, inwieweit unter dem geänderten Blickwinkel eine Revidierung der ursprünglich gewählten Strategie notwendig ist. Der Marktanteil ist dabei zum einen auf die Warengruppen und zum anderen auf die Filialgruppen zu beziehen. Eine derartige Portfolio-Matrix (vgl. Abbildung 18) läßt ähnliche Grundstrategien zu, wie das Image-Ertragskraft-Portfolio, wobei aber eine Positionsveränderung in vertikaler Richtung durch Marktanteilsstrategien ausgedrückt wird ${ }^{1}$.

Im betrachteten Beispiel weist das Portfolio des Warenhauses $\mathrm{X}$ recht viele Warengruppen mit unterdurchschnittlichem Marktanteil auf. Lediglich die Warengruppen Damenkleidung (1) und Schuhe und Kleintextilien (4) liegen deutlich über dem Durchschnitt aller Warengruppen. Für sie gilt eindeutig eine marktanteilsverteidigende Strategie und somit die gleiche Empfehlung wie bei der Analyse des ImageErtragskraft-Portfolios. Eine weitere Strategiekongruenz ergibt sich für die Warengruppe Verschiedener Bedarf (8):

1 Insofern erscheint diese Darstellung insbesondere als Vergleichs- oder Kontrollportfolio interessant. 
ihre hohe Ertragskraft legt die Erhöhung des Marktanteils z.B. durch Sortimentsausweitung, Verkaufsflächenerweiterung usw. nahe.

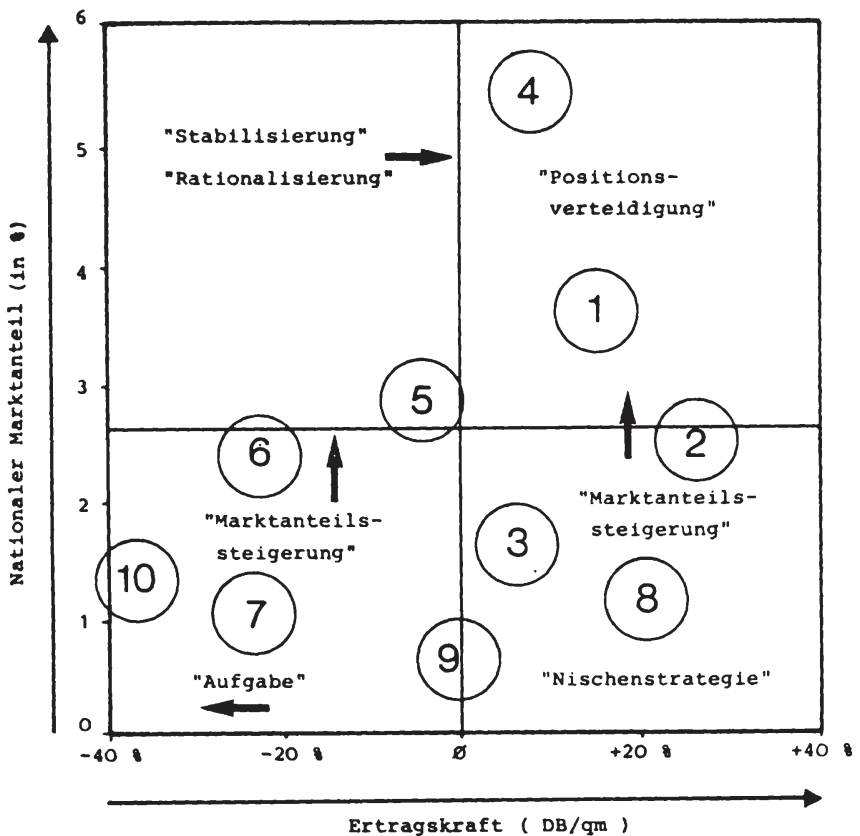

Abb. 18: Marktanteils-Ertragskraft-Portfolio der Warengruppen des Warenhauses $\mathrm{X}$

Für die Warengruppe Übriger Einrichtungsbedarf (6) zeigt das Portfolio einen fast durchschnittlichen Marktanteil und somit eine relativ günstige Wettbewerbssituation. Daher sind hier alle Anstrengungen, eventuell unter Inkaufnahme kurzfristiger weiterer Ertragsverschlechterungen, auf eine Verbesserung des Image zu legen. Damit trägt dieses Portfolio zu einer Absicherung der zuvor kaum begründba- 
ren Basisstrategie bei. Eine widersprüchliche Strategieempfehlung ergibt sich bei isolierter Betrachtung für die Warengruppe Lebensmittel (9). Während das sehr hohe Image eindeutig auf reine Rationalisierungsstrategien verweist, legt das Marktanteils-Ertragskraft-Portfolio als erste Priorität marktanteilssteigernde Aktivitäten nahe. Eine endgültige Entscheidung kann in einem solchen Konfliktfall erst nach weiteren Informationen bzw. PortfolioAnalysen getroffen werden.

Mit der Positionierung von Filialgruppen in das Marktanteil-Ertragskraft-Portfolio lassen sich aufgrund des ImageErtragskraft-Portfolios entwickelte Marketing-Basisstrategien um Konkurrenzaspekte ergänzen. Dies ist insbesondere für Filialgruppen mit unterdurchschnittlichem Image und unterdurchschnitilicher Ertragskraft interessant. Weisen sie gleichzeitig einen unterdurchschnittlichen Marktanteil auf, ist eine stufenweise Desinvestitionsstrategie angezeigt. Liegt jedoch eine ausreichende Wettbewerbsstärke vor, so sind eher positionsverbessernde Image und/oder Rationalisierungsstrategien angezeigt.

Der Vorteil des Marktanteils- Ertragskraft -Portfolios liegt insbesondere in den konkurrenzbezogenen strategischen Schlußfolgerungen begründet. Gerade auf dem konkurrenzintensiven Markt des Einzelhandels gewinnt dieser Aspekt an Bedeutung. Isoliert betrachtet führt das MarktanteilsErtragskraft-Portfolio zu ähnlichen Basisstrategien wie das Image-Ertragskraft-Portfolio, was auch durch die relativ große Ubbereinstimmung im Beispiel belegt wird. Allerdings impliziert die isolierte Betrachtung der Position einer strategischen Geschäftseinheit in dieser Matrix nur bedingt eine konkrete Strategie. So ist die Entscheidung über eine Marktanteilssteigerungs-Strategie nicht zuletzt davon abhängig, ob die Investitionen in einen wachsen- 
den, stagnierenden oder gar schrumpfenden Markt erfolgen. Dies ist durch zusätzliche Analysen zu überprüfen bzw. durch den Einsatz einer Portfolio-Variante festzustellen, die diese Fragestellungen explizit erfaßt.

\subsection{Warengruppen- und Filialgruppenstrategien auf der Basis des Marktanteils-Marktwachstums-Portfolios}

Das "klassische Marktanteils-Marktwachstums-Portfolid"1 positioniert die Warengruppen bzw. Filialgruppen in einer Matrix, deren Achsen durch den "relativen Marktanteil" und das "Marktwachstum" (real) gekennzeichnet sind.

Der "relative" Marktanteil bezeichnet eine Kennzahl aus dem Verhältnis des eigenen Marktanteils zum Marktanteil des nächstgrößeren Wettbewerbers bzw. des Marktführers ${ }^{2}$.

1 Entwickelt von der Boston-Consulting-Group. Vgl. zur Darstellung und Analyse z.B. Abell, D.F., Hammond, J.S., a. a.O., S. $173 \mathrm{ff.;}$ Aghte, K., Aktuelle Planungsprobleme eines internationalen Unternehmens, in: $\mathrm{ZfbF}, 1976, \mathrm{~S}$. 352-361, hier S. 357; Arbeitskreis "Langfristige Unternehmensplanung" der Schmalenbach-Gesellschaft, Strategische Planung, in: ZfB 29 1977, S. 1-20, hier S. 12 f.; Day, G.S., Diagnosing the Product Portfolio, in: JoM, Vol. 41, April 1977, S. 29-36; Hedley, B., A Fundamental Approach to Strategy Development, in: LRP Dez. 1976, S. 2-11; Derselbe, Strategy and the "Business Portfolio", in LRP, Febr. 1977, S. 9-15; Nagtegaal, H., a.a.O., S. 65 ff.; Cox, W.E., Product Portfolio Strategy: A Review of the Boston Consulting Group Approach to Marketing Strategy, in: AMA (Hrsg.) Combined Proceedings 1976, S. 465-470; Koch, H., Zum Verfahren der strategischen Programmplanung, in: ZfbF, 31 (1979), S. 145-161, hier S. 150 ff.; Roventa, P., a.a.O., S. 132-151; The Boston Consulting Group (Hrsg.), Das Portfolio, Perspektiven Nr. 19, Boston usw. 1977; Wind, Y., Product Portfolio Analysis: a New Approach to the Product Mix Decision, in: AMA (Hrsg.), Combined Proceedings 1976, S. 460-464; Wittek, B.F., a.a.O., S. 137 ff.; Ziegenbein, K., a.a.O., S. $72 \mathrm{ff} .$.

2 Dabei stellt das im folgenden diskutierte Beispiel nur auf die unmittelbaren Warenhauskonkurrenten ab. 
Der Marktanteil einer Warengruppe wird national gemessen und ergibt sich als eigener Umsatz in der Warengruppe $\mathrm{x}$ im Verhältnis zum gesamten Umsatz des institutionellen Einzelhandels in dieser Warengruppe. Ist der relative Marktanteil eines Warenhauses größer als der Wert 1, so ist das Warenhaus in diesem Bereich als Marktführer anzusehen. Das Marktwachstum wird real als Gesamtwachstum einer Warenoder Filialgruppe ermittelt. Bei Filialgruppen läßt es sich durch Aggregation der Wachstumsraten der einzelnen Regionen errechnen.

Die Einteilung der Matrix in vier Felder erfolgt in der Regel unternehmensspezifisch aufgrund von Plausibilitätsüberlegungen und läßt sich theoretisch kaum begründen. Normalerweise wird auf der relativen Marktanteilsachse der Schnitt bei einem Wert von 1,5 gelegt, womit ein um $50 \%$ höherer Marktanteil des betrachteten Unternehmens im Vergleich zum nächstgrößeren Wettbewerber markiert wird. Diese aus der Erfahrung in Industrieunternehmen resultierende Relation wird damit begründet, daß erst bei einem so deutlichen Marktanteilsvorsprung entsprechend große Kostenvorteile zu erwirtschaften sind, die eine lediglich marktpositionsverteidigende Strategie erlauben. Bei Warenhäusern ist jedoch aufgrund der oligopolistischen Struktur und der fehlenden Abhängigkeit von technologischen Innovationen eher ein Wert von 1,0 realistisch; denn angesichts der relativ knappen Kalkulation und der weitgehenden Ähnlichkeit der Sortimente ist ein relativer Marktanteil von knapp über 1,0 und damit die Marktführerschaft bereits ausreichend, um spürbare positive Kosteneffekte $z u$ erzielen.

Die horizontale Trennlinie orientiert sich am realisierten durchschnittlichen Marktwachstum aller Warengruppen Um die Vergleichbarkeit $z u$ sichern, ist ein Durchschnittswert über einen längeren Zeitraum (z.B. 5 Jahre) zu 
bilden ${ }^{1}$.

Die sich aus der Unterteilung ergebenden Matrixfelder werden üblicherweise mit den Begriffen "Question Mark", "Star", "Cash Cow" und "Dog" belegt (vgl. Abbildung 19) 2.

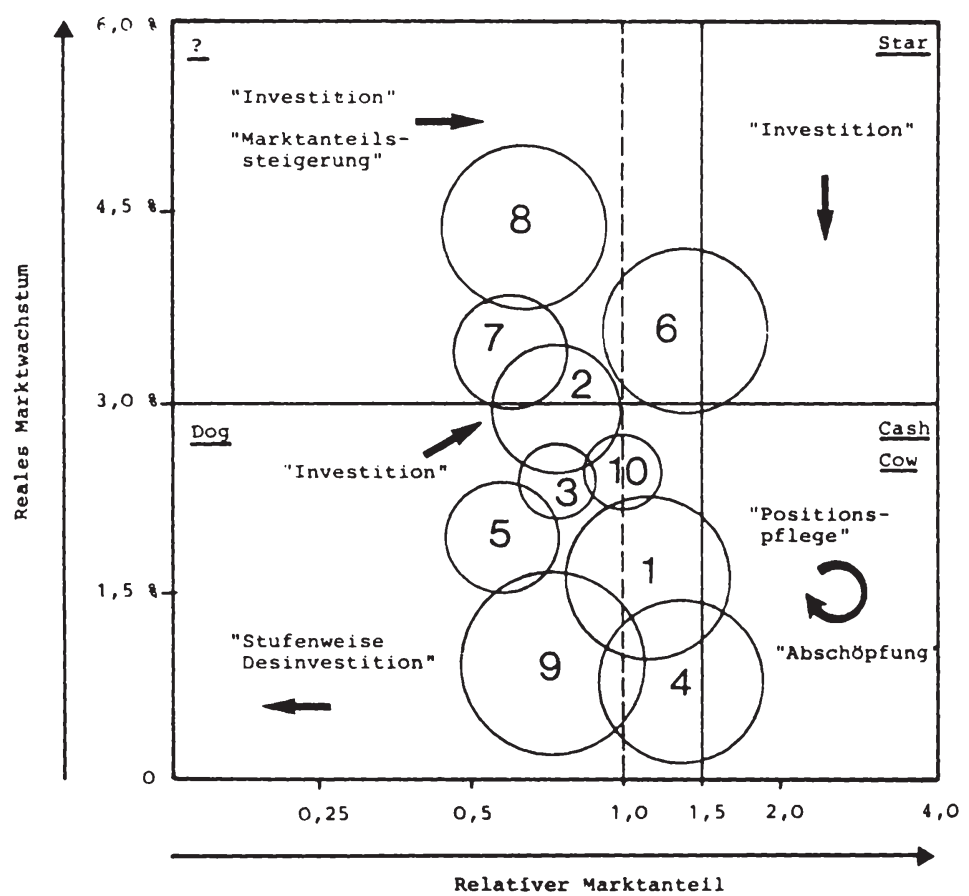

Abb. 19 : Marktanteils-Marktwachstums-Portfolio der Warengruppen des Warenhauses $\mathrm{X}$

1 Im betrachteten Beispiel des Warenhauses $\mathrm{x}$ ergibt sich entsprechend den vorliegenden Daten ein durchschnittliches Marktwachstum von 3\%.

2 Die Kreise bringen die Umsatzbedeutung der Warengruppen für das Warenhaus $x$ zum Ausdruck. 
Stars $^{1}$ beanspruchen als Warengruppen mit überdurchschnittlichem Marktwachstum und dem Potential zu dominierender Marktposition bis in die Reifephase i.d.R. sehr große finanzielle Ressourcen und erwirtschaften in der zeit des starken Wachstums kaum Finanzmittelüberschüsse. Denn das Halten einer Marktführerposition als Basisstrategie in stark wachsenden Bereichen zwingt zu ständigen Aktivitäten gegenüber den ebenfalls sehr aktiven Konkurrenten. Im skizzierten Beispiel läßt sich allenfalls die Warengruppe Übriger Einrichtungsbedarf (6) als Star bezeichnen. Doch hat sich ihr Marktwachstum bereits so stark verlangsamt, daß sie sich in den Cash-Cow-Bereich entwickelt. Damit rücken hier eindeutig marktanteilsverteidigende strategien in den Vordergrund. Ihre primäre Zielrichtung muß die in der Phase der Marktanteilssteigerung vernachlässigte Steigerung der Ertragskraft sein, was Rationalisierungsstrategien und eventuell preislagenkorrigierende Maßnahmen in den Mittelpunkt rückt.

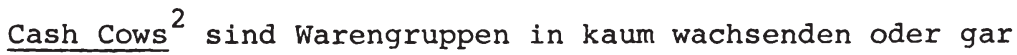
stagnierenden Märkten, für die gute Marktpositionen bestehen. Diese Warengruppen liefern die Finanzüberschüsse, die zum Aufbau der Nachwuchsgeschäfte und zur Sicherstellung der Stars benötigt werden. Cash Cows sichern kurzfristig den Erfolg der Unternehmung und stellen die Hauptquelle für Gewinne und Liquidität dar. Denn die Aktivitäten der Konkurrenten liegen in einem kaum wachsenden Markt i.d.R. auf einem relativ niedrigem Niveau. Damit sind für den Marktführer marktpositionssichernde Aktivitäten nur in geringem Umfang notwendig und die strategischen

1 Vgl. zur Beschreibung der Star-Position: The Boston Consulting Group (Hrsg.), The Star of the Portfolio, Perspektiven $\mathrm{Nr} .22$, München 1979.

2 Vgl. zur Beschreibung der Cash-Cow-Position: The Boston Consulting Group (Hrsg.), Anatomy of the Cash Cow, Perspektiven Nr. 25, München 1979. 
Aktivitäten primär darauf auszurichten, die Position zu pflegen und die Finanzmittelüberschüsse abzuschöpfen ${ }^{1}$. Im Beispiel sind für die Warengruppen Damenkleidung (1) und Schuhe und Kleintextilien (4) als Cash Cows des Warenhauses $\mathrm{X}$ die strategischen Stoßrichtungen der Positionspflege und Abschöpfung, die mit den bisher entwickelten Basisstrategien konsistent sind, $\mathrm{zu}$ verfolgen.

Question Marks nehmen mit einem geringen relativen Marktanteil - dabei aber ein hohes Marktwachstum - eine ambivalente Position ein: einerseits sind sie für die Unternehmung gefährlich, da sie aufgrund ihres starken Wachstums einen hohen Finanzmittelbedarf haben, ohne ihrerseits Finanzmittelüberschüsse zu erwirtschaften. Andererseits bieten Question Marks auch große Chancen, wenn ihr Marktanteil stark erhöht werden kann.

Damit bieten sich zwei alternative strategische Empfehlungen an:

(1) Die Erhöhung des Marktanteils durch intensive und differenzierte Marktbearbeitung, bevor das Wachstum sinkt. Man kann hier von "Investitionsstrategien" sprechen, die zum Ziel haben, die Question Marks mittelfristig zu Stars werden $\mathrm{zu}$ lassen.

(2) Besteht keine Möglichkeit zur Erhöhung des Marktanteils, so ist es zweckmäßiger, die Warengruppen aufzugeben (Desinvestitionsstrategien).

Im Warenhaus $\mathrm{x}$ nimmt lediglich die Warengruppe verschiedener Bedarf (8) die Question Mark Position ein. Für sie weisen die Ergebnisse der bisherigen Portfolio-Analysen eindeutig auf eine marktanteilssteigernde Basisstrategie hin. Insofern muß die Warenhausleitung prüfen, ob ein Teil des

1 Man spricht hier sehr anschaulich auch von einer "Erntestrategie". 
atypischerweise bereits erzielten Deckungsbeitrages unmittelbar in marktanteilssteigernde Marketing-strategien wie z.B. Sortimentsausweitung, Erhöhung der Personalintensität, Verkaufsflächenausweitung investiert werden sol1.

Schließlich werden als Dogs jene Warengruppen bezeichnet, die sowohl ein niedriges Marktwachstum als auch einen niedrigen Marktanteil aufweisen. Sie sind von ihrer Position nur durch unverhältnismäßig hohen Einsatz von Ressourcen in günstigere Positionen $z u$ bringen. Denn ihre relative Marktposition ist oftmals unzureichend, und es werden meist keine Finanzmittelüberschüsse erwirtschaftet, so daß jede Investition Gefahr läuft, in einer Falle ("cash trap") ohne Aussicht auf Gewinn zu verschwinden. Dies empfiehlt eine Desinvestitionsstrategie.

Im skizzierten Beispiel ist zwar keine Warengruppe einer eindeutigen Dog-Position zuzuordnen, allerdings liegen die Warengruppen Lebensmittel (9), textiler Einrichtungsbedarf (5), Alles für's Kind (3) und Herrenkleidung (2) in einer Utbergangsposition, die eine eindeutige strategiezuordnung erschwert. Zieht man zur Klärung Informationen über die Deckungsbeiträge und zum Umsatzvolumen hinzu, so kommt eine Desinvestitionsstrategie noch am ehesten. für die Warengruppen Textiler Einrichtungsbedarf (5) in Frage ${ }^{1}$. Bei der Warengruppe Lebensmittel (9) ist eher eine $\mathrm{Ab}-$ schöpfungsstrategie angezeigt, die unter Ausnutzung aller Rationalisierungsmöglichkeiten Finanzmittelüberschüsse erbringt. Für die Warengruppe Alles für's Kind (3) ist

1 Vor einer endgültigen Desinvestitionsentscheidung sind allerdings noch weiter Analysen z.B. über den Sortimentsverbund, Rationalisierungspotentiale usw. durchzuführen. 
eine Nischenstrategie empfehlenswert, durch die eine profilierende Mittellage erhalten werden kann.

Als erstaunlich gut erscheint hier die Position der Warengruppe Restaurant/Café (10). Allerāings ist die starke Wettbewerbsstellung kein Grund, die bisher als geeignet erachtete Strategie der Eliminierung $z u$ ändern: die schlechte Ertragslage trägt bei einer zukünftig chancenlosen Warengruppe lediglich $z u$ einer Kumulierung von Verlusten bei . Durch die Eliminierung könnten die hier mit Verlust eingesetzten Mittel vorteilhafter verwendet werden.

Insgesamt zeigt das Warengruppen-Portfolio ein relativ unausgewogenes Bild. Die Warengruppen weisen beim Marktanteilsvergleich mit dem Hauptwettbewerber eine geringe Streuung auf. Dies liegt in der oligopolistischen Marktstruktur der Warenhäuser und ihrer relativ festgefügten Marktposition begründet. Dennoch weist das MarktanteilsMarktwachstums-Portfolio für das Warenhaus $\mathrm{x}$ eindeutic eine Lücke in zukunftsträchtigen Warengruppen des QuestionMark- und Star-Bereiches aus. Folglich muß die Warenhausleitung die Suche nach zukunftsträchtigen Warengruppen intensivieren und ihre Aufnahme in das Sortiment realisieren.

Erstellt man das Marktanteils-Marktwachstums-Portfolio für Filialgruppen, so müssen die Schlüsselfaktoren aufgrunci einer regionalen Betrachtungsweise modifiziert werden. Die praktische Anwendung wirft dabei insbesondere Probleme bei der Bestimmung des Filialgruppen-Marktanteils auf. 
Zunächst sind die Marktanteile der einzelnen Filialen im regionalen Einzugs gebiet $z u$ bestimmen ${ }^{1}$. Diese sind im zweiten Schritt zu einem durchschnittlich-gewogenen Marktanteil der gesamten Filialgruppe zusammenzufassen ${ }^{2}$.

Bezieht man diesen Wert auf den (i.d.R. geschätzten) Marktanteil des jeweiligen Hauptwettbewerbers, erhält man den relativen Marktanteil der Filialgruppe ${ }^{3}$. Stellt man das (durchschnittliche) Marktwachstum entgegen, so lassen sich wiederum Basisstrategien entwickeln.

Filialgruppen im Question-Mark-Feld mit relativ hohem Marktwachstum und niedrigem relativen Marktanteil sind z.B. Filialgruppen, die in bevorzugten Standorten liegen, aber entweder gerade neu eröffnet oder von der Verkaursfläche her relativ begrenzt sind. Für diese Filialgrupper erscheint eine "Investitions"- bzw. "Ausbalistrategie" ancezeict. Durch marktanteilssteigernde Aktivitäten muß es gelingen, die relative Position gegenüber den Wettbewerbern zu verbessern, was allerdings

1 Dabei liefert z.B. die amtliche (örtliche) Steuerstatistik die zuverlässigsten Zahlen. Wo diese nicht aktuell vorliegen, müssen Schätzungen der örtlichen Warenhausleitung erste Richtlinien ergeben.

2 Die Gewichte der einzelnen Filial-Marktanteile ergeben sich dabei aus der Umsatzbedeutung des jeweiligen regionalen Einzugsgebiets im Verhältnis zu den anderen Einzugsgebieten der Filialen innerhalb einer Filialgruppe. Das Berechnungsverfahren vereinfacht sich für den Fall, daß eine Filialgruppe aus mehreren Einzelfilialen im gleichen regionalen Einzugsgebiet gebildet wird (z.B. Region Hamburg).

3 Für den Fall, daß Filialen aus unterschiedlichen standorten zu einer Filialgruppe zusammengefaßt werden, ist es denkbar, daß an jedem Standort zwar ähnliche absolute Marktanteile vorliegen, die relativen Marktanteile aufgrund der eventuell unterschiedlichen Konkurrenzstruktur jedoch erhebliche Differenzen aufweisen. Dann erscheint es sinnvoll, statt des relativen den gewichteten absoluten Marktanteilswert heranzuziehen. 
ihre besondere Berücksichtigung bei der Zuteilung finanzieller Mittel voraussetzt.

Filialgruppen im Star-Feld weisen mit ihrem relativ hohen Marktwachstum und ihrem hohen Marktanteil in bevorzugten wachstumsstarken Märkten eine starke Wettbewerbsstellung auf. Hier muß es das primäre Ziel der Warenhausleitung sein, die Partizipation am Marktwachstum durch wettbewerbsvorsprungssichernde Aktivitäten wie z.B. Profilierungsstrategien, intensive Kommunikation, Sortimentsaktualisierung, zu erhalten. Diese werden ebenso wie die Strategien im Question-Mark-Feld relativ hohe Finanzmittel beanspruchen.

Die Filialgruppen im Cash-Cow-Feld weisen einen hohen Marktanteil bei niedrigem Marktwachstum auf. Diese Filialgruppen sind in den jeweiligen Regionen im Konkurrenzgefüge fest etabliert und erzeugen jene Finanzmittelüberschüsse, die für die investitionsorientierten Strategien benötigt werden. Für sie gelten primär Abschöpfungs-, Positionspflege- oder -erhaltungs-, bzw. Konsolidierungsstrategien. Auf der Basis der günstigen Wettbewerbssituation gilt es, z.B. durch Rationalisierungsstrategien, ertragsorientierte Sortimentssteuerung und profilierende Kommunikationsstrategien die finanziellen Filialgruppenüberschüsse zu sichern bzw. noch zu erhöhen.

Für Filialgruppen im Dog-Bereich liegt aufgrund ihres niedrigen Marktwachstums, ihres niedrigen Marktanteils und aufgrund ihrer damit schwachen Wettbewerbsposition die stufenweise Desinvestition nahe. Dabei können diese Filialgruppen insgesamt bzw. zunächst einzelne Filialen innerhalb der Filialgruppen geschlossen werden, um die dadurch freiwerdenden Ressourcen anderen zukunftsträchtige- 
ren Filialgruppen zukommen zu lassen. Angesichts der aktuellen Warenhauskrise erscheint diese Basisstrategie durchaus auch in der Realität interessant. So haben in den letzten drei Jahren alle Warenhauskonzerne einzelne unrentable und chancenlose Filialen aufgegeben.

Das Marktwachstums-Marktanteils-Portfolio erlangt zum einen durch seine unmittelbare Orientierung am Hauptwettbewerber und zum anderen durch die Berücksichtigung finanzieller Aspekte wesentliche Aussagekraft. Diese wird weiterhin durch die implizite Berücksichtigung der zukünftigen Chancen der Waren- bzw. Filialgruppen in Form des Marktwachstums verbessert. Speziell für Warenhäuser ergibt sich bei der Anwendung dieses Portfolios das Problem, daß aufgrund der oligopolistischen, seit Jahren festgefügten Konkurrenzsituation, viele strategische Geschäftseinheiten in Ubbergangslagen positioniert sind, denen nur bedingt eine portfoliospezifische Basisstrategie zuzuordnen ist ${ }^{1}$. Hierzu sind zusätzliche Informationen aus anderen Portfolios notwendig. 2

Als letztes analytisches Portfolio wird das Marktwachstums-Unternehmenswachstums-Portfolio, das nunmehr explizit das Umsatzwachstum des eigenen Warenhauses über mehrere Jahre hinweg berücksichtigt, dargestellt.

1 Vgl. zur generellen Kritik an dieser Portfolio-Variante z.B. Day, G.S., a.a.O., S. 36 ff.; Wittek, B.F., a.a.O., S. 144 .

2 So weist z.B. die Warengruppe Herrenkleidung (2) eine relativ ungünstige Lage im Portfolio auf, die entweder starke Investitionen zur Erlangung der Cash-CowPosition oder eine Abschöpfungsstrategie verlangt. Dagegen weisen die bisherigen Portfolios jedoch eindeutig darauf hin, daß hier offensichtlich eine lukrative Nische durch das Warenhaus $x$ besetzt wird, die es auch zukünftig zu pflegen gilt. 
4.24 Warengruppen- und Filialgruppenstrategien auf der Basis des Marktwachstums-UnternehmenswachstumsPortfolios

Das Martkwachstums-Unternehmenswachstums-Portfolio stellt das durchschnittliche Marktwachstum der vergangenen 5 Jahre dem Unternehmenswachstum der jeweiligen Warengruppen und Filialgruppen im gleichen Zeitraum gegenüber.

Auf der $45^{\circ}$-Linie (vgl. Abbildung 20 ) liegen jene Warengruppen oder Filialgruppen, die weder einen Verlust oder Gewinn von Marktanteilen aufweisen; das durchschnittliche

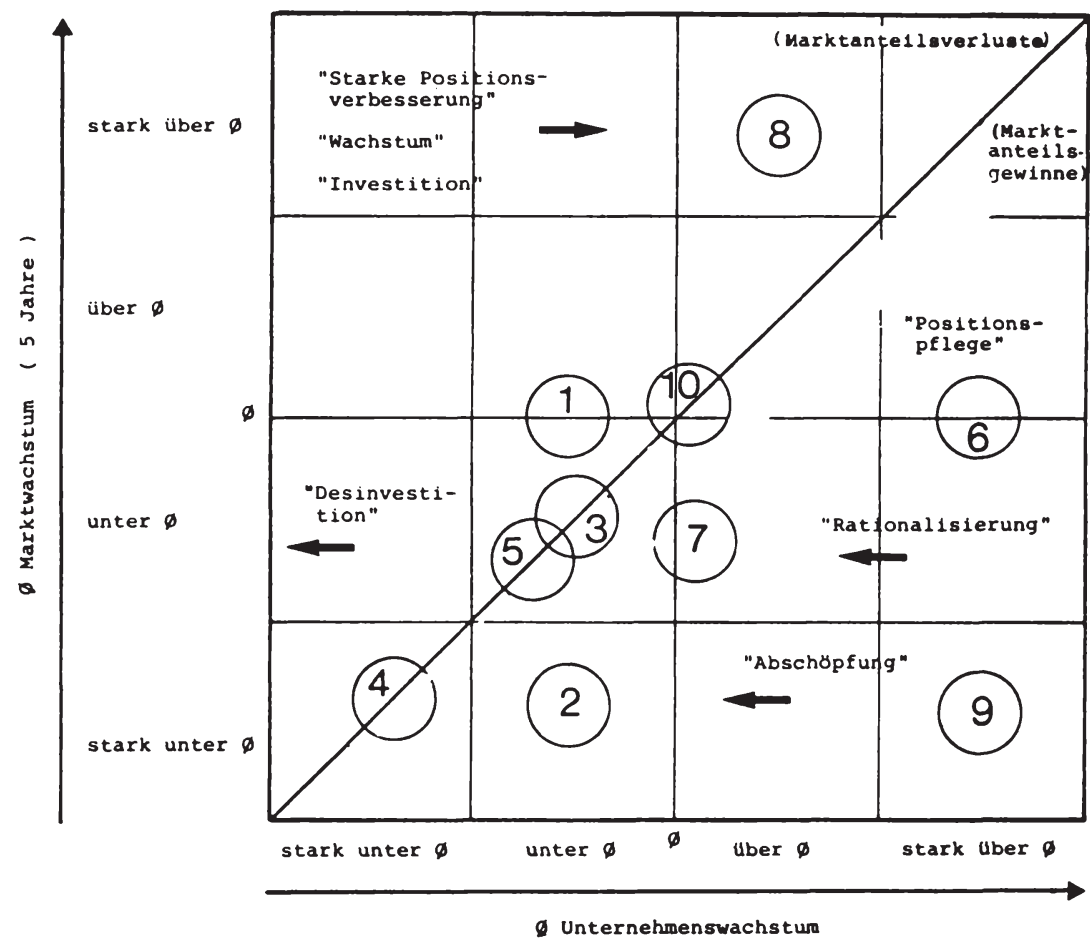

Abb.20: Wachstums-Portfolio der Warengruppen des Warenhauses $\mathrm{X}$ 
Unternehmenswachstum ist mit dem durchschnittlichen Marktwachstum identisch. Der Bereich oberhalb der $45^{\circ}$-Linie weist auf Marktanteilsverluste hin, der Bereich unterhalb entsprechend auf Marktanteilsgewinne. Bei der zentralen Bedeutung des Marktanteilsziels muß es für ein Warenhaus als strategisch anstrebenswert erscheinen, Positionen unterhalb von der $45^{\circ}$-Linie zu erreichen, damit schneller als der Markt zu wachsen und die Marktposition auszubauen ${ }^{1}$.

Liegen die positionierten Warengruppen im attraktiven Wachstumsfeld (überdurchschnittliches Marktwachstum), so sind bei einer Lage oberhalb der $45^{\circ}$-Linie Wachstumsbzw. Investitionsstrategien angezeigt, da ein zukünftiges langsameres Unternehmenswachstum Marktanteilsverluste mit sich bringt. So zeigt das Portfolio für die Warengruppe Damenkleidung (1), daß die bislang stets sehr gute Positionierung zukünftig nur durch Investitions- und Wachstumsstrategien wie z.B. Verkaufsflächenerweiterung, Erhöhung der Personalintensität usw. auch gehalten werden kann. Ohne Änderung der bisherigen Basisstrategien würde die erreichte Cash-Cow-Position langfristig in Gefahr sein.

Für die Warengruppe Verschiedener Bedarf(8) wird bestätigt, daß die Warenhausleitung eine Entscheidung zwischen einer Nischenstrategie und einer Marktanteilssteigerungs- und damit einer Positionsverbesserungsstrategie treffen muß. Diese Entscheidung wird deshalb relativ schwer zu treffen sein, weil diese Warengruppe über eine hohe Ertragskraft verfügt und eine Positionsverbesserungsstrategie zwangsläufig den kurz- bzw. mittelfristigen

1 Die mit Hilfe dieses Portfolios abgeleiteten Basisstrategien sind mit ähnlichen Bezeichnungen belegt wie die bisherigen, so daß im folgenden wenige Hinweise genügen. 
Verzicht auf freigesetzte Mittel bedeuten würde. Allerdings erscheint unter langfristigen Perspektiven nur auf diese Art und Weise die Star-bzw. Cash-Cow-Position - basierend auf einer starken Wettbewerbsposition und einem hohen Image - erreichbar ${ }^{1}$.

Im Fall des unattraktiven Wachstums (unterdurchschnittlichen Marktwachstum) ist bei einer Lage oberhalb der $45^{\circ}$ Linie eine Desinvestitionsstrategie zu überprüfen, da in einem weitgehend stagnierenden Markt Anstrengungen, die eine starke Verbesserung der Marktanteile - gegen den Trend - anstreben, wenig sinnvoll sind ${ }^{2}$. Liegt das eigene Wachstum deutlich über dem Marktwachstum, so ist im Hinblick auf den stagnierenden Markt zu überprüfen, ob zur Aufrechterhaltung des erreichten Marktanteils nicht geringere Investitionen ausreichen bzw. die Ertragskraft der Warengruppen durch eine Verringerung der Marktinvestition erhöht werden kann. Damit würde zwar das Unternehmenswachstum zurückgehen, jedoch immer noch über dem Marktwachstum liegen und sich somit zur optimalen Positionierung $^{3}$ hin bewegen.

1 Gerade hier wird der positive Effekt der mehrstufigen analytischen Portfolio-Planung deutlich. Die isolierte Betrachtung der Warengruppe 8 , die bei den ersten Portfolios eine Nischenstrategie impliziert, wird durch die Gesamtbetrachtung mehrerer Portfolios, die jeweils unterschiedliche "Schnitte" durch die Unternehmens-MarktBeziehungen legen, revidiert und tendenziell in eine Marktanteilssteigerungs- bzw. Positionsverbesserungsstrategie modifiziert.

2 In diesem Bereich sind keine Warengruppen des Warenhauses $x$ positioniert.

3 Dieser Bereich gilt deshalb als "optimal", weil hier mit vergleichsweise geringen Investitionen der Vorsprung gegenüber den Wettbewerbern kaum spürbar, aber stetig vergrößert wird. Dadurch ist die Gefahr verstärkter Aktivitäten seitens der Wettbewerber, die wiederum starke eigene Aktivitäten erfordern, relativ gering. 
So hat das Warenhaus $\mathrm{x}$ in den vergangenen Jahren offensichtlich deutlich stärker in die Warengruppe Lebensmittel (9) investiert als die übrigen Warenhauskonkurrenten und damit eine azyklische Politik realisiert. Angesichts des nahezu stagnierenden Marktes scheint nun - in Konsistenz zu den übrigen Portfolios - der übergang zu einer Abschöpfungsstrategie angezeigt.

Auch für die Warengruppe Übriger Einrichtungsbedarf (6) ergibt sich eine konsistente strategieempfehlung: das starke Unternehmenswachstum ist beizubehalten, die Wettbewerbsposition weiter zu verbessern. Bei langfristig sinkenden Wachstumsraten werden dadurch die Voraussetzungen für die Entwicklung zur Cash-Cow geschaffen.

Positioniert man Filialgruppen in das MarktwachstumsUnternehmenswachstums-Portfolio, so verdeutlichen die erreichten Positionen die Konkurrenzstellung im jeweiligen Regionalmarkt, wobei auf dieser Betrachtungsebene das durchschnittliche Marktwachstum regional zu ermitteln ist. Da die zu entwickelnden Basisstrategien weitgehend mit den Warengruppen-Strategien identisch sind, soll hier auf eine detaillierte Beschreibung verzichtet werden.

Insgesamt verlangt diese Portfolio-Variante insbesondere als Ergänzungs- bzw. Kontrollportfolio Bedeutung. Sie erweitert die bisherige Betrachtungsweise um den Aspekt des Unternehmenswachstums, dem zumindest in der vergangenen Dekade in Warenhäusern höchste Priorität zukam. Isoliert betrachtet ist dieser Variante nur eine begrenzte Aussagekraft zuzuordnen, einerseits, weil im oligopolistischen Warenhausmarkt relativ viele Warengruppen auf der $45^{\circ}$-Linie positioniert sind und andererseits, weil die einseitige Markt- und Unternehmens- Wachstumsorientierung durch die fehlende Ertrags- und Kundenorientierung evtl. zu falschen strategischen Empfehlungen führt. 
Leiteten sich die Dimensionen der dargestellten analytischen Portfolios aus einzelnen Zielen der Warenhäuser sowie aus dem Kunden- und Konkurrenzbezug ab, sind im folgenden Portfolios zu analysieren, deren Schlüsseldimensionen auf der Basis individueller Einschätzung durch ein Mehrfaktorensystem gekennzeichnet sind.

\subsection{Anwendung synthetischer Portfolio-Varianten in Warenhäusern}

\subsection{Warengruppen- und Filialgruppenstrategien auf der Ba- sis des Lebenszyklus-Wettbewerbspositions-Portfolios}

Die Marktchancen einer Waren- bzw. Filialgruppe sind wesentlich durch ihre stellung im Lebenszyklus, die die generellen wachstumsaussichten erfaßt, sowie durch ihre Wettbewerbsposition, die die unternehmensspezifischen stärken wiedergibt, determiniert. Damit liegt dem LebenszyklusWettbewerbspositions-Portfolio ${ }^{1}$ zunächst eine ähnliche Ubberlegung wie dem Marktwachstums-Unternehmenswachstums-Portfolio zugrunde. Als synthetisches Portfolio enthalten seine Achsendimensionen aber wesentlich umfassendere Schlüsselfaktoren.

Die Wahl der Achsendimension "Wettbewerbsposition" resultiert aus der Überlegung, daß der notwendige Konkurrenzbezug der strategischen Planung nicht alleine auf dem

1 Dieses Portfolio wird zu Beratungszwecken hauptsächlich von der Firma A.D. Little eingesetzt. Vgl. zur Darstellung Albach, H., Unsicherheit, a.a.0., S. 708 f.; Patel, P., Younger, M., A Frame of Reference for Strategy Development, in: LRP, April 1978, S. 6-12; Thanheiser, H. und Patel, P., a.a.O., S. 65; Stoff, W.D., a.a.O., S. 8 ff.; Wittek, B.F., a.a.O., S. $151 \mathrm{ff.}$. 
relative Marktanteil begründet sein sollte. Vielmehr wird die Wettbewerbsposition zusätzlich z.B. durch das Unternehmens- und Marktwachstum, die Stabilität der Marktanteilsverteilung, die Sortimentsbreite und-tiefe, das Image usw. bestimmt ${ }^{1}$. Jede strategische Geschäftseinheit ist nun im Hinblick auf diese Kriterien mit dem Hauptwettbewerber $\mathrm{zu}$ vergleichen. Dabei wird im vorliegenden Beispiel einer einfachen Heuristik gefolgt, die auf eine (quasi-) analytische Verdichtung der Einzelbeurteilungen - wie sie z.B. bei detaillierten Punktbewertungsmodellen $^{2}$ erfolgt - verzichtet. Vielmehr wird eine qualitative Einschätzung in einer mehrstufigen Skala vorgenommen, deren spektrum von "dominant" bis "schwach" reicht ${ }^{3}$. Die strategischen Geschäftseinheiten werden dabei lediglich anhand der Kriterien Marktanteil, Image und Preisführer/folgerschaft durch subjektive Einschätzung des Managements festgelegt ${ }^{4}$.

Eine dominante Wettbewerbsposition ist für ein Warenhaus in einer bestimmten Warengruppe bzw. Filialgruppe dann gegeben, wenn es eindeutig als Marktführer zu charakterisieren ist.Dies impliziert, daß es den größten Umsatz und damit auch

1 Selbstverständlich sind je nach Branche auch andere $\mathrm{Ab}-$ stufungen denkbar.

$2 \mathrm{Vgl}$. zur Beschreibung einer solchen Vorgehensweise das Marktattraktivitäts-Wettbewerbsvorteile-Portfolio.

3 Im Falle unterschiedlicher Einzelurteile können die Einzelbeurteilungen gewichtet werden. Diese Gewichtung ist natürlich subjektiv vorzunehmen und kann generell nicht festgelegt werden. Im obigen Beispiel bietet sich für den Marktanteil ein Gewicht von 0,4 , für das Image und die Preisführer/-folgerschaft jeweils 0,3 an. 
den größten Marktanteil mit deutlichem Vorsprung vor dem nächst größeren Warenhausunternehmen aufweist, daß die Kundenpräferenz eindeutig bei ihm liegt und daß es die Preisführerschaft innehat. Eine starke Wettbewerbsposition ist durch eine nicht eindeutige Marktführerschaft gekennzeichnet. Dies bedeutet einen geringen Marktanteilsvorsprung im Vergleich zum wichtigsten Warenhaus-Wettbewerber, der zudem ständig bedroht ist. Das Image des Warenhauses beim Kunden ist zwar relativ gut, doch führen ertragssteigernde Preiserhöhungen zu Marktanteilsverlusten. Im Bereich der schlechteren Wettbewerbsstellung ist eine Differenzierung in haltbar und schwach vorzunehmen. Als haltbar wird eine Position bezeichnet, bei der das Warenhaus nachrangiger Anbieter ist, d.h. einen geringeren Marktanteil als der Marktführer aufweist und sich mit der Rolle des Preisfolgers zufrieden geben muß. Ein Warenhaus verfügt schließlich über eine schwache Wettbewerbsposition, wenn es nur einen sehr niedrigen Marktanteil innehat und seine Konkurrenten die Bewegungen am Markt "diktieren". Als letzte Möglichkeit ergibt sich eine gleiche Wettbewerbsposition im Vergleich zum Hauptwettbewerber.

Die zweite Achsendimension, die Stellung im Lebenszyklus resultiert aus der überlegung, daß die zukünftige Marktentwicklung einer Waren-bzw. Filialgruppe nicht alleine aus ihrem zurückliegenden Marktwachstum hergeleitet, sondern daß hierzu vielmehr auf die umfassenderen Erkenntnisse des Lebenszykluskonzeptes zurückgegriffen werden sollte ${ }^{2}$. Deswegen werden im vorliegenden Portfolio die fünf Lebenszyklusphasen Einführung, Wachstum, Reife, Sättigung und Verfall unterschieden, in die die Warenbzw. Filialgruppen zu positionieren sind.

1 Diese Begriffsinterpretation geht über die reine Marktanteilsbetrachtung hinaus. Vgl. hierzu auch Kotler, P., a.a.O., S. 272 ff.. 
Auf der Basis der Schlüsselfaktoren "Wettbewerbsposition" und "Position im Lebenszyklus" läßt sich die in Abbildung 21 dargestellte Portfolio-Matrix aufstellen.

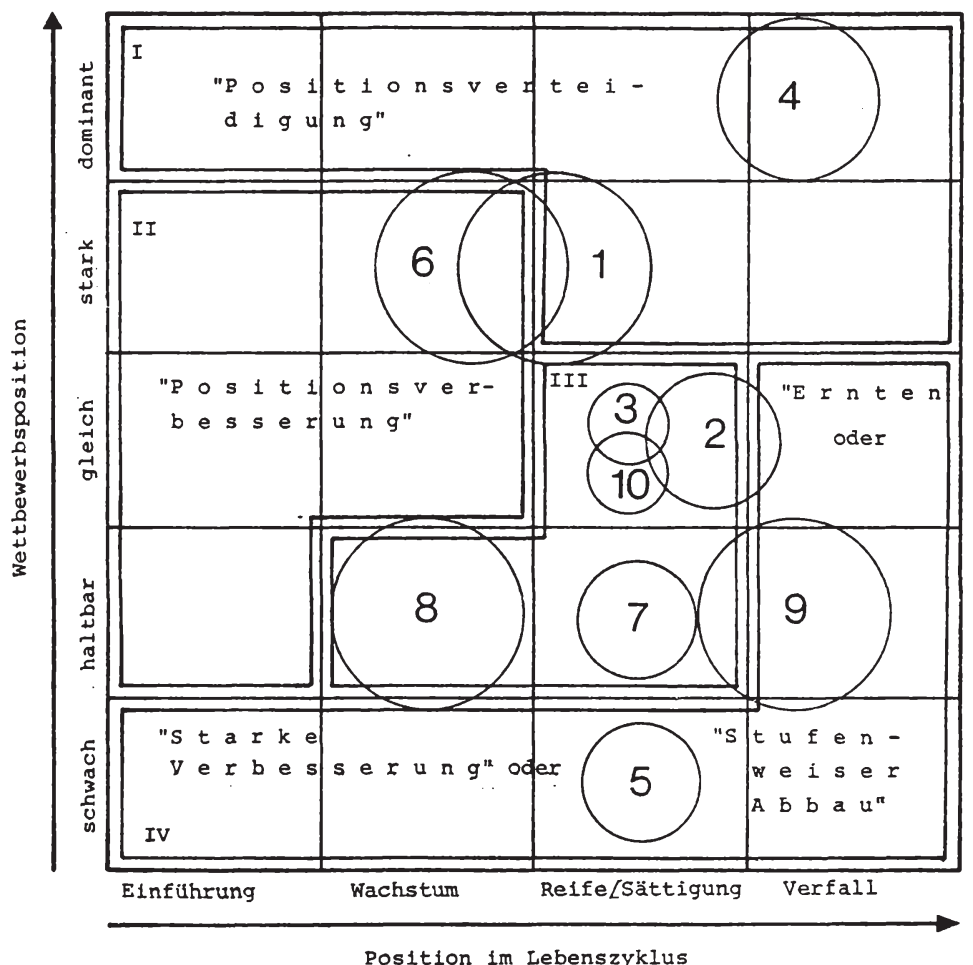

Abb. 21 : Lebenszyklus-Wettbewerbspositions-Portfolio der Warengruppen des Warenhauses $\mathrm{x}$

Im Rahmen der Warengruppenbetrachtung muß bei einer dominanten Wettbewerbsposition (Feld I) z.B. die Warengruppe Damenkleidung (1) als eindeutiger Marktführer ihre Position durch eine Sortiments- und Preisführerstrategie, durch die Erschließung neuer Zielgruppen und durch den Ausbau des Imagevorsprungs halten. Selbstverständlich 
erhalten dabei einzelne Strategieelemente je nach der Position im Lebenszyklus eine andere Bedeutung. So rücken bei der Warengruppe Schuhe und Kleintextilien (4), die sich nach Einschätzung des Managements am Beginn der Verfallsphase befindet, zunehmend Rationalisierungsstrategien in den Vordergrund. Hier gilt es, die erreichte Position mit geringstmöglichem Aufwand zu halten, um möglichst hohe Finanzmittelüberschüsse zu erzielen.

Im Feld II sind junge Warengruppen anzutreffen. Die zu entwickelnden Basisstrategien müssen darauf abzielen, eine Positionsverbesserung $z u$ erreichen und dementsprechend durch geeigneten Marketing-Instrumenteeinsatz möglichst nahe an den Marktführer heranzukommen. Diese Strategien erforciern in der Regel hohe Investitionen, die jedoch getätigt werden müssen, da diese Warengruppen das Geschäft von morgen darstellen. Das Beispiel des Warenhauses $x$ zeigt hier eine eindeutige Lücke, da die Warengruppe übriger Einrichtungsbedarf (6) fast das Feld I erreicht hat.

Für Warengruppen im Feld III wird die Erzielung einer generellen Marktführerposition als aussichtlos beurteilt. Hier bietet sich eine Marktnischenstratesie an, d.h. das Warenhaus spezialisiert sich auf einen bestimmten Sortiments- bzw. Bedürfnisausschnitt und erwirbt sich dadurch eine spezifische Kompetenz, die auch vom Kunden honoriert wird. Im Beispiel des Warenhauses $\mathrm{x}$ weist diese Portfolio-Variante auf eine Nischenstrategie bei den Warengruppen Herrenkleidung (2), Alles für's Kind (3), Restaurant/Café (10), Einrichtungszubehör (7) sowie Verschiedener Bedarf hin. Diese Basisstrategie ist weitgehend konsistent $z u$ den bislang entwickelten strategischen Stoßrichtungen. Allerdings ist für die Warengruppe Einrichtungszubehör (7) auch eine stufenweise Desinvestition in Verbindung mit einer Erntestrategie zu erwägen.

Für die Warengruppen im Feld IV sind dann Eliminierungsstrategien zu empfehlen, wenn sie sich in einem fortge- 
schrittenen Lebenszyklusstadium befinden. Im Hinblick auf die ertragsorientierten Zielsetzungen ist für Warengruppen, die sich bei schwacher Wettbewerbsposition bereits in der Sättigungs- oder Verfallsphase befinden, vielfach die stufenweise Aufgabe richtig. Im Beispiel betrifft dies die Warengruppe Textiler Einrichtungsbedarf (5), die bislang stets eine "unscharfe" Mittelposition einnahm. Dieses Portfolio deckt nunmehr die zu schwache und $\mathrm{zu}$ wenig chancenreiche Position auf, so daß eine Abschöpfungsstrategie platzgreifen kann. Für die Warengruppe Lebensmittel (9) ergibt sich eine ähnliche Situation. Hier muß die Warenhausleitung entscheiden, ob die bislang - entgegen dem Markttrend - realisierte Forcierung auch zukünftig fortgesetzt werden soll.

Wendet man das Lebenszyklus-Wettbewerbspositions-Portfolio für Filialgruppen an, so ist die Wettbewerislage identisch zu bestimmen. Bezüglich des Lebenszyklus ist dagegen für Filialgruppen eine von der Warengruppenbetrachtung differierende Interpretation notwendig. Zwar durchlaufen einzelne Filialen eine (Umsatz-) Entwicklung, die dem im Produktlebenszyklus gekennzeichneten Verlauf ähnlich ist; sie erreichen aber nach einer meist relativ kurzen Einführungsund Wachstumsphase eine i.d.R. sehr lange Reife- bzw. Sättigungsphase. Ist in einer bestimmten Region das örtliche Marketingkonzept erst einmal durchgesetzt, so weist es aufgrund der oftmals vorhandenen starken regionalen wettbewerbsposition ein starkes Beharrungsvermögen auf und entwickelt sich meist nur aufgrund drastischer Veränderungen auf der Konkurrenz- oder auf der Kundenseite zur Verfallsphase hin ${ }^{1}$.

1 Es darf nicht verkannt werden, daß sich gegen diese Art der idealtypischen Beschreibung der Entwicklung einer Filiale über ihren gesamten Existenzzeitraum hinweg nahezu die gleichen Argumente wie gegen das ProduktlebenszyklusKonzept vorbringen lassen.Vgl. hierzu u.a. Dhalla,N.K., Yuspeh, S., a.a.O., S. $69 \mathrm{ff}$. sowie die Erwiderung von: Birkigt, K., Kritische Anmerkungen zu Dhalla und Yuspeh, in: Harvard Manager, Heft 1/1980, S. 79-82. 
Ordnet die Warenhausleitung alle Filialgruppen in die Phasen eines solchen idealtypischen Lebenszyklusmodells ein und analysiertgleichzeitig die jeweilige Wettbewerbslage, so lassen sich aus der gesamtunternehmensbezogenen Sicht wesentliche Erkenntnisse für die strategischen Stoßrichtungen ableiten. Im Hinblick auf die Basisstrategien ist insbesondere auf jene Filialgruppen zu achten, die im Lebenszyklus relativ weit fortgeschritten sind und gleichzeitig eine schwache Wettbewerbslage aufweisen. Aufgrund ihrer relativ hohen Kapitalbindung ist für solche Filialgruppen eine stufenweise Aufgabe zu überprüfen. Dadurch könnten unmittelbar Finanzmittel freigesetzt werden, die notwendig sind, um besser positionierte Filialgruppen zu unterstützen. Zum anderen ist die Ausgewogenheit der Filialgruppen im Portfolio insofern zu überprüfen, als prinzipiell eine möglichst breite verteilung der Filialgruppen über den gesamten Lebenszyklus - mit Schwerpunkt in der Reife/Sättigungsphase - zu realisieren ist, damit der Bestand des Unternehmens langfristig gesichert werden kann. Dabei sind insbesondere die Felder in der mittleren Wettbewerbslage durch Neugründungen zu besetzen, damit die zukünftig unter ertragsorientierten Gesichtspunkten notwendigen Desinvestitionsstrategien realisiert und gleichzeitig ihre negativen Auswirkungen auf den Marktanteil bzw. die Marktstellung kompensiert werden können.

Insgesamt betrachtet ist die Berücksichtigung dieser Aspekte, zweifellos positiv zu werten. Sind die Kennzahlen in ihrer Entwicklung realistisch $z u$ schätzen sowie die Phasen relativ exakt abzugrenzen, so visualisiert der Lebenszyklus die zukünftigen Chancen für Warengruppen oder Filialgruppen. Zugleich wird mit der Konkurrenzdimension durch die Analyse der Wettbewerbslage ein zentraler strategischer Schlüsselfaktor berücksichtigt. 
Besonders kritisch ist hierbei die Verwendung des Lebenszykluskonzeptes als solches $z$ u betrachten. Die berechtigte Kritik an der Grundhypothese des Gesetzes vom "Werden und Vergehen" läßt die Einbeziehung der Lebenszyklusposition in das Portfolio von Warenhäusern grundsätzlich bedenklich erscheinen: da keine hinreichende Absicherung des Konzeptes vorliegt, besteht die Gefahr, aufgrund falscher Voraussetzungen wenig sinnvolle strategien abzuleiten. Diese sind zwar unter formalen Gesichtspunkten folgerichtig entwickelt, können jedoch evtl. wegen der unrichtigen Prämissen eine für das Gesamtsystem nicht förderliche Richtung empfehlen. Darüber hinaus ist gegen die beschriebene Bestimmung der Wettbewerbsposition die mehrfach notwendige subjektive Schätzung und Gewichtung durch das Management vorzubringen. Dies läßt sich auch durch weitere analytische Verfeinerung der Beurteilung $z$.B. in einem Punktbewertungsverfahren nicht lösen. Hinzu kommt, daß sich durch diese Vorgehensweise positive und negative Einzelfaktoren ausgleichen. Diese Nivellierungsgefahr kann leicht zu einer "analytischen Unschärfe" führen", d.h. Gründe für eine bestimmte Positionierung und damit auch die strategischen Ansatzpunkte $\mathrm{zu}$ ihrer Veränderung werden umso weniger deutlich, je mehr Faktoren Berücksichtigung finden. Man versucht ihr durch adäquate Operationalisierungs- und Generierungsverfahren $\mathrm{zu}$ begegnen, was insbesondere für das zusätzliche Faktoren berücksichtigende - Marktattraktivitäts-Wettbewerbsvorteile-Portfolio gilt.

1 Vgl. Wittek, B.F., a.a.O., S. 154. 
4.32 Warengruppen- und Filialgruppenstrategien auf der Basis des Marktattraktivität-Wettbewerbsvorteile-Portfolios

Das Marktattraktivität-Wettbewerbsvorteile-Portfolio ${ }^{1}$ beruht zum einen auf der Überlegung, daß sich die strategische Planung einer Unternehmung nicht auf zwei Dimensionen zurückführen läßt, sondern vielmehr auf einem Konglomerat ganzer Einflußfaktorenbündel basiert, die sowohl quantitative als auch qualitative Größen berücksichtigen. Zum anderen wird es durch ein Mehrfaktorensystem möglich, unternehmensspezifisch relevante Faktoren zu berücksichtigen, wodurch nicht zuletzt die Ansichten des Managements über die Relevanzbestimmung stärkeres Gewicht erhalten ${ }^{2}$.

Erstellt man dieses Portfolio für die Waren- oder Filialgruppen von Warenhäusern, so sind zunächst die Faktoren der Marktattraktivität und der relativen ${ }^{3}$ Wettbewerbs-

1 Abell, D.F., Hammond, J.S., a.a.O., S. 122 ff.; Albach, H., Unsicherheit, a.a.O., S. $706 \mathrm{ff}$.; Ax, A., Börsig, C., a.a.O., S. $118 \mathrm{ff.;}$ Clifford, D.K., Bridgewater, B.A., Hardy, T., The Game has Changed, in: The McKinsey Quarterly, Herbst 1975, S. 2-21; Ebskamp, K., Strategische Planung in der Praxis, ZfbF-Kontaktstudium 31 (1979), S. 11-20, hier S. 13 f.; Hussey, D.W., Portfolio Analysis: Practical Experience with the Directional Policy Matrix, in: LRP, August 1978, S. 2-8; Lorange, P., Vancil, R.F., Strategic Planning Systems, Englewood Cliffs N.J., 1977, S. 172-186; Robinson,S.J.,Hichens, R.E., Wade, D.P., The Directional Policy Matrix-Tool for Strategic Planning, in: LRP, June 1978, S. 8-15; Roventa, P., a.a.O., S. 151 ff.; Springer,C.H., Strategic Management in General Electric, in: Operations Research 1973, S. 1177-1182; Szyperski, U., Winand, U., a.a.O., S. 127 f.; Wittek, B.F., a.a.O., S. $144 \mathrm{ff}$..

2 Dies erscheint insbesondere deshalb wichtig, weil die Durchsetzung der entwickelten Strategien durch das Management erfolgen muß. Diese wird umso problemloser möglich sein, je höher die Identifikation mit der Planungsmethodik und damit den entwickelten Basisstrategien ist. Vgl. zum Aspekt der Durchsetzungsproblematik z.B. Smit, J., Rade, N.L., Rational and Non-Rational Planning, in: LRP, Vol. 13, April 1980, S. 87-101.

3 Der Zusatz "relativ" bringt zum Ausdruck, daß die Faktoren jeweils im Vergleich zum stärksten Konkurrenten im jeweiligen Marktsegment $z u$ analysieren sind. 
vorteile festzulegen. Aufgruna von Plausibilitätsüberlegungen sind für Warenhäuser die in der Tabelle 5 wiedergegebenen Faktoren von Relevanz ${ }^{1}$.

\section{Faktoren der Marktattraktivität}

- Marktwachstum

- Marktgröße

- durchschnittliche Rentabilität

- Stellung im Marktlebenszyklus

- Wettbewerbsintensität

- Einkaufsbesonderheiten

- Beschaffungssicherheit

- Konjunkturabhängigkeit

- Abhängigkeit von der Gesetzgebung

Faktoren der relativen Wettbewerbsvorteile

- absoluter Marktanteil

- relativer Marktanteil

- Unternehmensgröße und Finanzkraft

- Unternehmensrendite

- Unternehmenswachstumsrate

- Marktposition (Marktführer oder -folger)

- Image

- Standortvorsprung (Anzahl und Qualität)

- Qualität des Managements

- Flexibilität des Planungssystems

Tab. 5 : Relevante Faktoren der Marktattraktivität und relativen Wettbewerbsvorteile für Warenhäuser

Anschließend sind diese Faktoren von der Warenhausleitung subjektiv zu gewichten. Die Tabelle 6 zeigt auszugsweise eine Gewichtung, die jedem Subfaktor einen Wert zwischen $O$ und 100 zumißt, der seiner prozentualen Bedeutung innerhalb der Gesamtheit der Faktoren entspricht.

1 Diese Einschätzung beruht auf der subjektiven Erfahrung von Warenhaus-Managern. 


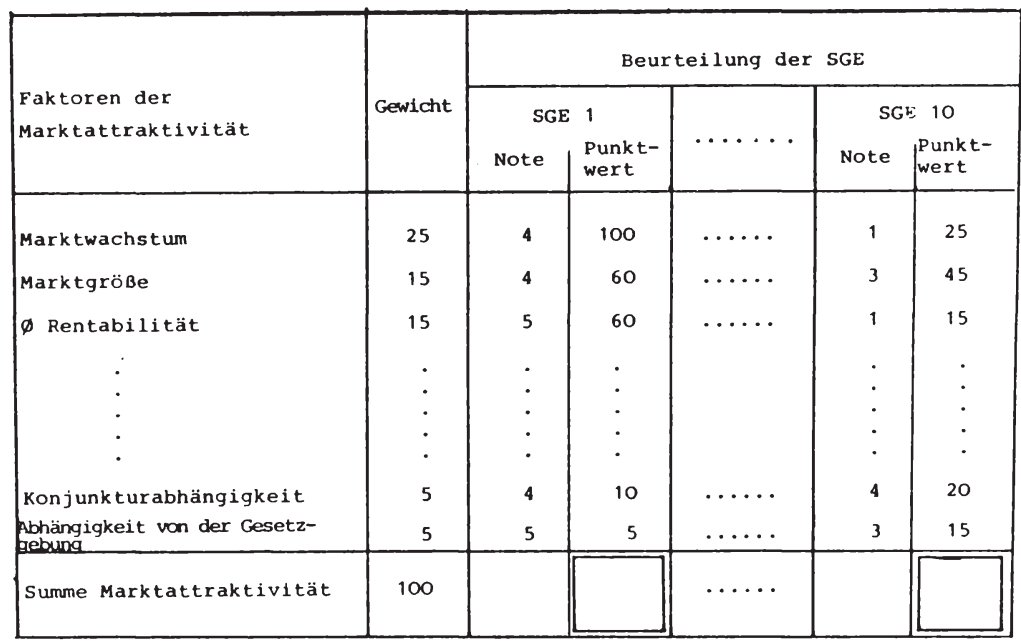

Tab. 6: Schema eines Punktbewertungsverfahrens zur Beurteilung der Marktattraktivität

Jede strategische Geschäftseinheit wird nun anhand jedes einzelnen Faktors beurteilt. Dabei hat es sich als praktikabel erwiesen, Noten zwischen 1 (= unwichtig, schlecht, gering etc.) und 5 (= wichtig, gut, hoch etc.) zu geben ${ }^{1}$, diese mit dem Faktorgewicht zu multiplizieren und die jeweiligen Einzelpunktwerte für das betrachtete Beispiel in eine Skala mit den Ausprägungen niedrig - mittel - hoch einzutragen. Geht man bei der 2. Dimension ebenso vor, so sind im diskutierten Beispiel die Warengruppen des Warenhauses $\mathrm{X}$ wie in der Abbildung 22 positioniert.

Demnach hat das Warenhaus $x$ mit der Warengruppe Damenkleidung (1), die eindeutig im Feld der Wachstums- und Investi-

1 Die Messung der Beurteilungsdimension ist dabei für jeden Faktor unterschiedlich. So ist z.B. der Faktor Marktwachstum in Prozentwerten und die Marktgröße in DM-Beträgen zu messen, wogegen die Abhängigkeit von der Gesetzgebung nur in qualitativen Urteilen (wie z.B. hoch - mittel - niedrig) ausdrückbar ist. Analog hierzu muß in einem sog. "Notenschlüssel" angegeben werden, bei welcher Ausprägung (z.B. of Wachstum) welche Noten z.B. 1 (= Stagnation), 2 (= leichtes Wachstum = 1-3 8 ) usw., erteilt werden muB. 
tionsstrategien positioniert ist, die Marktführerschaft in einem attraktiven Markt erobert, die es zu halten und auszubauen gilt. Die Warengruppe Herrenkleidung (2) ist diesem Feld nur bedingt zuzurechnen. Tendenziell weist ihre Position auf eine weitere Verstärkung der Wettbewerbsvorteile z.B. durch Profilierungs- und Marktanteilssteigerungsstrategien hin.

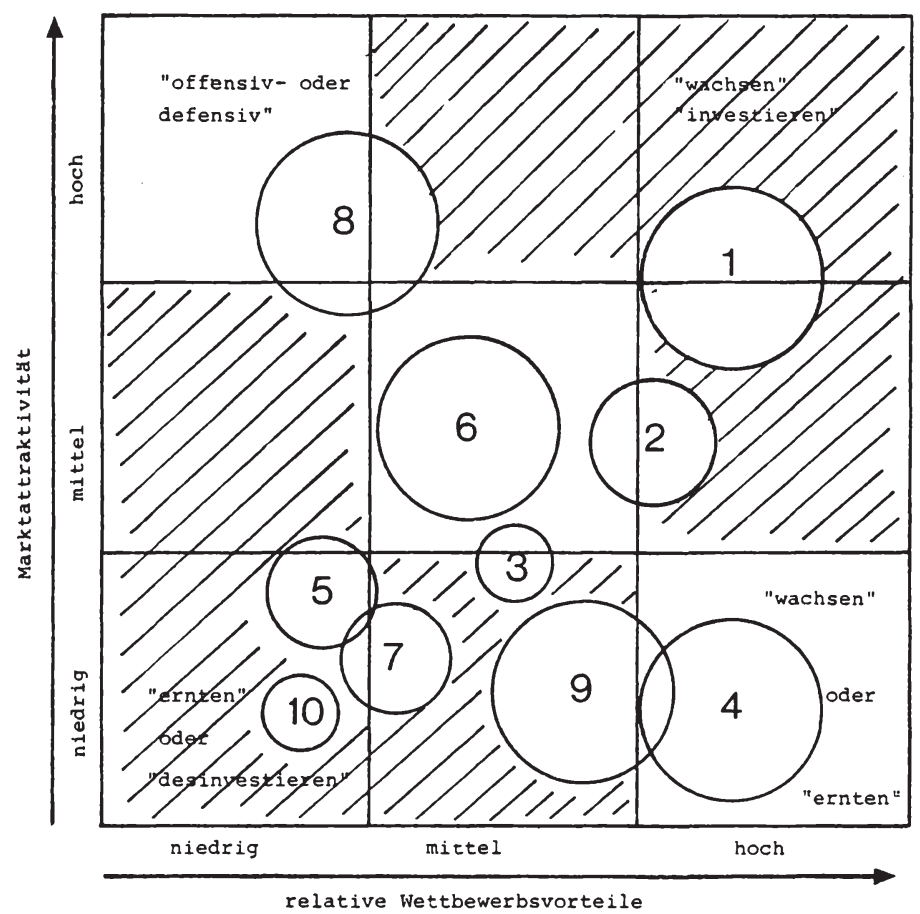

Abb. 22 : Marktattraktivitäts-Wettbewerbsvorteile-Portfolio der Warengruppen des Warenhauses $\mathrm{X}$

Für die Warengruppen Alles für's Kind (3), Textiler Einrichtungsbedarf (5), Einrichtungszubehör (7), Lebensmittel (9) und Restaurant/Café (10)liegen die strategischen Stoßrichtungen "ernten" oder "desinvestieren" nahe. 
Dabei ist insbesondere für die Warengruppe Lebensmittel (9) aufgrund ihrer großen Umsatzbedeutung eine Erntestrategie z.B. durch Rationalisierungsmaßnahmen, Verkaufsflächenrückgang usw. empfehlenswert.

Für die (unschraffierten) Felder, die eine Mittellage bzw. eine hoch/niedrig Kombination kennzeichnen ${ }^{1}$, ist die Strategieempfehlung problematisch. Die Warengruppe Verschiedener Bedarf (8) weist z.B. eine hohe Marktattraktivität, aber niedrige relative Wettbewerbsvorteile auf. Hier muß die Warenhausleitung entscheiden, ob sie die Marktchancen nutzen und durch starke Investitionen versuchen soll, die Wettbewerbsvorteile auszubauen, oder ob sie die Warengruppe im Wege einer Defensivstrategie entweder aufgeben oder in eine Nischenposition bringen will. Die Entscheidung für eine Basisstrategie kann dabei nicht alleine aufgrund der Portfolio-Positionierung getroffen werden. Ein Rückgriff auf die Einzelbeurteilung der berücksichtigten Faktoren weist jedoch wegen der guten Ertragskraft, der günstigen Lebenszyklusphase und den Wachstumsraten auf eine Wachstums- und Investitionsstrategie hin.

Die typische Mittellage der Warengruppe Ujbriger Einrichtungsbedarf (6) läßt keine Entwicklung einer Basisstrategie zu. Auch hier mu auf die Einzelbeurteilung der Faktoren oder auf weitere Analysen zurückgegriffen werden.

Für eine Position, die durch hohe relative Wettbewerbsvorteile und niedrige Marktattraktivität gekennzeichnet

1 In der McKinsey-Terminologie werden diese Felder als Bereiche der "Selektivstrategien" bezeichnet, was letzten Endes bedeutet, daß eine eindeutige Stoßrichtung aus der position selbst nicht ersichtlich ist. 
ist, wie sie die Warengruppe Schuhe und Textile Einrichtungen (4) einnimmt, werden als alternative Basisstrategien Wachstums- oder Erntestrategien empfohlen. Greift man auf die Einzelbeurteilungen zurück, so ist hier eine Basisstrategie angezeigt, die aufgrund der erreichten Wettbewerbsvorteile zur Erzielung möglichst hoher Finanzmittelüberschüsse beiträgt.

Positioniert man Filialgruppen in das MarktattraktivitätsWettbewerbsvorteil-Portfolio, so ist die Vorgehensweise und die Entwicklung von Basisstrategien grundsätzlich ähnlich. Es ist jedoch sinnvoll, die den Warengruppen zugrundegelegten Faktoren zum einen regionalspezifisch $\mathrm{zu}$ interpretieren und festzulegen. Zum anderen ist im Einzelfall vom Management zu prüfen, ob nicht einzelne Faktoren gestrichen, durch andere ersetzt oder auch umgewichtet werden sollten. So rücken z.B. bei der Marktattraktivität Faktoren der regionalen Wirtschaftsstruktur (Bevölkerungszusammensetzung, Kaufkraft), städtebauliche Restriktionen, Flächenverfügbarkeit usw. stärker in den Vordergrund, während z.B. die Konjunkturabhängigkeit oder die Beschaffungssicherheit weniger bedeutsam werden.

Insgesamt ist dieses Portfolio für die Entwicklung von Filialgruppenstrategien durch die unmittelbare Konkurrenzorientierung der Basisstrategien bedeutsam. Basierend auf der detaillierten überprüfung der Konkurrenzlage sind die entwickelten Basisstrategien unmittelbar gegen die Wettbewerber gerichtet, denn jede Basisstrategie, die auf eine Verbesserung der Wettbewerbsvorteile ausgerichtet ist, setzt am Aufbau von Stärken bzw. an der Bewahrung von bereits erzielten Vorteilen an. Gleichzeitig kann damit die Gesamtverteilung der Filialgruppen im Hinblick auf ihre finanzielle Lage überprüft werden. Dabei muß das Warenhaus über eine genügend große Anzahl von Filial- 
gruppen im bereich "ernten" verfügen, da jene die Finanzmittel freisetzen, die für den Ausbau der Wettbewerbsvorteile bei den anderen Filialgruppen benötigt werden.

Die Verwendung vielfältiger Faktoren eines Marktattraktivitäts-Wettbewerbsvorteile-Portfolios fördert zusammenfassend betrachtet die Aussagekraft der entwickelten Basisstrategien, da die strategierelevanten situativen Variablen vollständiger erfaßt sind und unmittelbare strategische Ansatzpunkte durch die Einzelbeurteilung sichtbar werden. Hier wird jedoch zugleich auch die Grundproblematik dieser Variante ${ }^{1}$ deutlich: die Faktoren sind subjektiv festzulegen $^{2}$, zu gewichten und additiv zu verknüpfen. Damit ist die intersubjektive Nachprüfbarkeit der Positionierung nicht mehr gegeben. Weiterhin besteht dadurch die Gefahr, daß die Positionierung der strategischen Geschäftseinheiten nicht eindeutig ist ${ }^{3}$, da sich sehr gute und sehr schlechte Einzelurteile ausgleichen können und damit strategische Geschäftseinheiten in Mittelpositionen gedrängt werden. Die Entwicklung von Basisstrategien ist in solchen Fällen nur noch unter $\mathrm{Z}$ uhilfenahme der Einzelinformationen möglich.

\subsection{Zusammenfassung und kritische Würdigung der Eignung der Portfolio-Methode zur Entwicklung von Basisstra- tegien in Warenhäusern}

\section{41 Vergleich der isoliert entwickelten Marketing-Basis- strategien für die Warengruppen des Warenhauses $\mathrm{X}$}

In den vorangegangenen Ausführungen wurden die verschiede-

1 Vgl. zur kritischen würdigung z.B. Koch, M., Selektive Programmplanung, a.a.O., S. 151 ff.; Jünger, P., a.a.O., S. $107 \mathrm{ff.;}$ Wittek, B.F., a.a.O., S. $147 \mathrm{ff} .$.

2 Die PIMS-Studie, die teilweise zur Begründung herangezogen wird weist nur für einen Teil der üblicherweise herangezogenen Faktoren den Bezug zum Unternehmenserfolg nach.

3 Vgl. hierzu Wittek, B.F., a.a.O., S. 148. 
nen Portfolio-Varianten weitgehend isoliert zur Entwicklung von Marketing-Basisstrategien für Waren- bzw. Filialgruppen eingesetzt. Bereits bei dieser isolierten Diskussion wurde deutlich, daß die aus der Positionierung resultierenden Marketing-Basisstrategien für die Warengruppen des Warenhauses $\mathrm{x}$ in den verschiedenen Portfolio-Varianten nicht immer übereinstimmen. Dies ist darauf zurückzuführen, daß bei der analytisch-sukzessiven Portfolio-Methode jeweils nur zwei Schlüsselfaktoren berücksichtigt werden, bei Betrachtung anderer Schlüsselfaktoren aber zusätzliche Aspekte ins spiel kommen, aufgrund derer andere strategische Schlubfolgerungen $z u$ ziehen sind. Bei der synthetischen Portfolio-Methode sind dagegen divergierende Basisstrategien mit der unterschiedlichen Art, Anzahl und Gewichtung der berücksichtigten Faktoren zu erklären.

Somit stellt sich für die Warenhausleitung die Aufgabe, die isoliert entwickelten Marketingstrategien auf ihre Konsistenz zu überprüfen und eine endgültige Entscheidung über die $z u$ verfolgende Basisstrategie zu treffen. Hier$\mathrm{zu}$ ist im Rahmen des stetigen Rückkoppelungsprozesses ${ }^{1}$ auf die vorab grob festgelegten Rahmenentscheidungen zurückzugreifen $^{2}$, wobei insbesondere der strategieevaluierenden ${ }^{3}$ Funktion der Marketingziele Rechnung zu tragen ist. Falls eine eindeutige Strategie aus den Portfolios nicht erkennbar ist, müssen die Alternativen anhand der in der Marketing-zielsetzungen zum Ausdruck kommenden Prioritäten bewertet und dementsprechend eine Auswahl getroffen werden ${ }^{4}$.

$1 \mathrm{Vgl}$. hierzu die Ausführungen im Punkt 1.22.

2 Diese werden im Verlauf des Planungsprozesses durch Erkenntnisse über Strategiemöglichkeiten stets weiter konkretisiert.

3 Die strategiegenerierende Funktion der Marketingziele wurde u.a. durch die Verwendung der Ziele als Schlüsselfaktoren demonstriert.

4 Denkbar ist auch eine Neudefinition und Neubewertung der Marketingziele. 
Ebenso sind die Strategiealternativen im Hinblick auf ihre Vereinbarkeit mit den Positionierungsentscheidungen $\mathrm{zu}$ überprüfen. Im Konfliktfall ist jene Basisstrategie vorzuziehen, die am ehesten der Positionierung gegenüber den Käufersegmenten und den Konkurrenten entspricht ${ }^{1}$.

Für das Beispiel des Warenhauses $\mathrm{x}$ weisen die isoliert entwickelten Marketing-Basisstrategien für die meisten Warengruppen einen erstaunlich hohen Grad an Ubereinstimmung auf und unterscheiden sich vielfach nur durch die unterschiedlichen strategiebegriffe. Insgesamt resultieren aus den sechs verschiedenen Portfolio-Varianten die in den Tabellen 7, 8 und 9 festgehaltenen Marketing-Basisstrategien $^{2}$. Sie verdeutlichen abschließend beispielhaft den Zusammenhang zwischen der Position einer Warengruppe und den zu entwickelnden Basisstrategien.

Diese relativ gute tubereinstimmung der strategien erlaubt jedoch keinesfalls eine Verallgemeinerung. Vielmehr wird der reale Anwendungsfall häufig durch Inkonsistenzen bzw. Konflikte im entwickelten Strategiensystem gekennzeichnet sein, da die Positionierungen bei unterschiedlichen Portfolios (bzw. Schlüsselfaktoren) stark divergieren können. Da der theoretisch exakte Lösungsansatz aufgrund einer übergeordneten "Nutzenfunktion" nicht praktikabel erscheint, bietet sich als Heuristik die mehrfach rückgekoppelte sukzessive Entwicklung von Strategien an. Diese umfaBt zum einen die sukzessive Bearbeitung der einzelnen Planungsphasen, wobei nach jedem Schritt eine Rückkoppelung $z u$ den vorangegangenen Phasen mit einer eventuellen Modidikation der getroffenen Entscheidungen erfolgt. Zum anderen betrifft diese speziell die Entwicklung von Basisstrategien auf der Basis der Portfolio-Methode.

1 Denkbar ist auch eine Modifizierung der bisherigen (Ausgangs-) Positionierung.

$2 \mathrm{Da}$ sie in den vorangegangenen Kapiteln ausführlich diskutiert wurden, soll die Zusammenfassung in den Tabellen nicht mehr weiter erläutert werden. 


\begin{tabular}{|c|c|c|c|c|}
\hline \multirow{2}{*}{ Waren } & \multicolumn{2}{|c|}{$\begin{array}{c}\text { Image/Ertragskraft } \\
\text { Portfolio }\end{array}$} & \multicolumn{2}{|c|}{$\begin{array}{c}\text { Marktanteil/Ertragskraft- } \\
\text { Portfolio }\end{array}$} \\
\hline & Position & & Position & \\
\hline 1 & sehr gut & Positionsverteidigung & sehr gut & Positionserhaltung \\
\hline 2 & sehr gut & Positionsverteidigung & gut & Marktanteilssteigerung \\
\hline 3 & gut & Positionsverteidigung & mittel & $\begin{array}{l}\text { Marktanteilssteigerung/ } \\
\text { Nischenpolitik }\end{array}$ \\
\hline 4 & gut & $\begin{array}{l}\text { Positionsverbesserung/ } \\
\text {-verteidigung }\end{array}$ & sehr gut & Positionserhaltung \\
\hline 5 & schlecht & Positionsverbesserung & mittel & Rationalisierung \\
\hline 6 & $\begin{array}{l}\text { sehr } \\
\text { schlecht }\end{array}$ & Positionsverbesserung & schlecht & Marktanteilssteigerung \\
\hline 7 & $\begin{array}{l}\text { sehr } \\
\text { schlecht }\end{array}$ & $\begin{array}{l}\text { Positionsverbesserung/ } \\
\text { Aufgabe }\end{array}$ & $\begin{array}{l}\text { sehr } \\
\text { schlecht }\end{array}$ & Aufgabe/Nischenpolitik \\
\hline 8 & schlecht & $\begin{array}{l}\text { Nischenpolitik/Posi- } \\
\text { tionsverbesserung }\end{array}$ & mittel & $\begin{array}{l}\text { Nischenpolitik/Markt- } \\
\text { anteilssteigerung }\end{array}$ \\
\hline 9 & gut & $\begin{array}{l}\text { Positionsverteidigung/ } \\
\text { Rationalisierung }\end{array}$ & schlecht & Nischenstrategie \\
\hline 10 & $\begin{array}{l}\text { sehr } \\
\text { schlecht }\end{array}$ & $\begin{array}{l}\text { Aufgabe/starke Posi- } \\
\text { tionsverbesserung }\end{array}$ & $\begin{array}{l}\text { sehr } \\
\text { schlecht }\end{array}$ & Aufgabe \\
\hline
\end{tabular}

Tab. 7: Isolierte Entwicklung der Marketing-Basisstrategien mit Hilfe des ImageErtragskraft-Portfolios und des Marktanteils-Ertragskraft-Portfolios 


\begin{tabular}{|c|c|c|c|c|}
\hline \multirow{2}{*}{$\begin{array}{l}\text { Waren- } \\
\text { gruppe }\end{array}$} & \multicolumn{2}{|c|}{$\begin{array}{l}\text { Marktanteils- Marktwachs- } \\
\text { tums - Portfolio }\end{array}$} & \multicolumn{2}{|c|}{$\begin{array}{l}\text { Marktwachstum- } \\
\text { Unternehmenswachstums- } \\
\text { Portfolio }\end{array}$} \\
\hline & Position & Basisstrategie & Position & Basisstrategie \\
\hline 1 & cash Cow & Abschöpfung & MA-Verluste & Positionsverbesserung \\
\hline 2 & Mittellage & Wachstum & MA-Gewinne & Abschöpfung \\
\hline 3 & $\begin{array}{l}\text { Mittellage/ } \\
\text { Dog }\end{array}$ & $\begin{array}{l}\text { Wachstum/stufen- } \\
\text { weise Desinvesti- } \\
\text { tion }\end{array}$ & durchschnittl & $\left\{\begin{array}{l}\text { halten/leichte Posi- } \\
\text { tionsverbesserung }\end{array}\right.$ \\
\hline 4 & Cash Cow & Abschöpfung & durchschnittl & $\{$ halten/Desinvestition \\
\hline 5 & Dog & Desinvestition & durchschnittl & $\begin{array}{l}\text { Abschöpfung/stufenwei- } \\
\text { se Desinvestition }\end{array}$ \\
\hline 6 & star & $\begin{array}{l}\text { Marktanteilssteige- } \\
\text { rung/Investition }\end{array}$ & MA-Gewinne & Positionspflege \\
\hline 7 & Question Mark & $\begin{array}{l}\text { Marktanteilssteige- } \\
\text { rung/Investition }\end{array}$ & MA-Gewinne & Abschöpfung \\
\hline 8 & Question Mark & $\begin{array}{l}\text { Marktanteilssteige- } \\
\text { rung/Investition }\end{array}$ & MA-Verluste & Positionsverbesserung \\
\hline 9 & Dog & $\begin{array}{l}\text { ernten/stufenweise } \\
\text { Desinvestition }\end{array}$ & MA-Gewinn & Abschöpfen \\
\hline 10 & Mittellage & $\%$ & durchschnittl & $\left\{\begin{array}{l}\text { halten/leichte Posi- } \\
\text { tionsverbesserung }\end{array}\right.$ \\
\hline
\end{tabular}

\section{MA=Marktanteil}

Tab. 8 : Isolierte Entwicklung der Marketing-Basisstrategien mit Hilfe des Marktanteils-Marktwachstums-Portfolio und des Marktwachstums-UnternehmenswachstumsPortfolios 


\begin{tabular}{|c|c|c|c|c|}
\hline \multirow{2}{*}{ Waren- $\begin{array}{c}\text { Portfolio- } \\
\text { Varian- } \\
\text { te }\end{array}$} & \multicolumn{2}{|c|}{$\begin{array}{c}\text { Lebenszyklus-Wettbewerbs- } \\
\text { Portfolio }\end{array}$} & \multicolumn{2}{|c|}{$\begin{array}{c}\text { Marktattraktivitäts-Wettbewerbs- } \\
\text { vorteile-Portfolio }\end{array}$} \\
\hline & Position & Basisstrategie & Position & Basisstrategie \\
\hline 1 & gut & Positionsverteidig. & sehr gut & $\begin{array}{l}\text { Wachsen, Investieren, } \\
\text { Position sichern }\end{array}$ \\
\hline 2 & mittel & Nischenpolitik & mittel/gut & $\begin{array}{l}\text { Nischensuche mit Ten- } \\
\text { denz:Investieren }\end{array}$ \\
\hline 3 & mittel & Nischenpolitik & $\begin{array}{l}\text { mittel/ } \\
\text { schlecht }\end{array}$ & $\begin{array}{l}\text { Nischensuche mit Ten- } \\
\text { denz:ernten }\end{array}$ \\
\hline 4 & $\begin{array}{l}\text { gut/sehr } \\
\text { gut }\end{array}$ & Positionsverteidig. & $\begin{array}{l}\text { mittel/ } \\
\text { schlecht }\end{array}$ & $\begin{array}{l}\text { Defensivstrategie: } \\
\text { halten und ernten }\end{array}$ \\
\hline 5 & schwach & Aufgabe & schlecht & ernten,Desinvestition \\
\hline 6 & gut & $\begin{array}{l}\text { Positionsverbesse- } \\
\text { rung }\end{array}$ & mittel & $\begin{array}{l}\text { Selektivstrategie } \\
\text { (mit Tendenz: Investieren) }\end{array}$ \\
\hline 7 & $\begin{array}{l}\text { mittel/ } \\
\text { schwach }\end{array}$ & Nischenpolitik & schlecht & ernten, Desinvestition \\
\hline 8 & $\begin{array}{l}\text { mittel/ } \\
\text { schwach }\end{array}$ & Nischenpolitik & mittel & $\begin{array}{l}\text { Offensivstrategie: } \\
\text { wachsen, investieren }\end{array}$ \\
\hline 9 & schwach & Aufgabe & $\begin{array}{l}\text { mittel/ } \\
\text { schlecht }\end{array}$ & ernten \\
\hline 10 & mittel & Nischenpolitik & schlecht & ernten, Desinvestition \\
\hline
\end{tabular}

Tab. 9: Isolierte Entwicklung der Marketing-Basisstrategien mit Hilfe des LebenszyklusWettbewerbs-Portfolio und des Marktattraktivitäts-Wettbewerbsvorteile-Portfolios 
Vergleicht man die einzelnen Basisstrategien sukzessiv 1 und zieht somit nach und nach immer mehr Faktoren in die Analyse mit ein, so ermöglicht diese Vorgehensweise eine Abwägung bzw. Bewertung der isoliert abgeleiteten Marketing-Basisstrategien. Hierdurch gelingt es der Warenhausleitung, insgesamt ein konsistentes system von Warengruppenstrategien $\mathrm{zu}$ entwickeln, das einerseits allen relevanten strategiebeeinflussenden Faktoren sowohl isoliert als auch in Kombination untereinander erfaßt und andererseits das "Zusammenpassen" der Strategien in ihrer Gesamtheit erreicht. Die solchermaßen entwickelten Basisstrategien für die Warengruppen 1-10 sind in der Tabelle 10 festgehalten.

Die Feststellung, daß sich durch die verschiedenartige Anwendung der Portfolio-Methode - ausgedruickt durch alternative, sukzessiv zu vergleichende Portfolio-Varianten insgesamt konsistente und damit sinnvolle Marketing-Basisstrategien für Warenhäuser ableiten lassen, qualifiziert dieses Verfahren im Hinblick auf seine inhaltliche Aussagekraft für den Einsatz in Warenhäusern. Als zentrale Planungsmethode bei der Ableitung von Basisstrategien muß es darüber hinaus aber auch in der Lage sein, die Integrationsfunktion der Marketing-Basisstrategien im Rahmen der strategischen Marketingplanung in Warenhäusern zu unterstützen. Das ist im folgenden kritisch zu überprüfen.

1 Dabei sind auch die synthetischen Portfolio-Varianten in den Prozeß der sukzessiven Strategieentwicklung mit einbezogen. 


\begin{tabular}{|c|c|}
\hline $\begin{array}{l}\text { Waren= } \\
\text { gruppe }\end{array}$ & Marketing-Basisstrategien \\
\hline 1 & $\begin{array}{l}\text { Positionsverteidigungsstrategie durch } \\
\text { - Imagewahrungs- } \\
\text { - Marktanteils- und } \\
\text { - Ertragskraftsicherungsaktivitäten }\end{array}$ \\
\hline 2 & $\begin{array}{l}\text { Abschöpfungsstrategie durch } \\
\text { - Rationalisierungs- und } \\
\text { - Spezialisierungsaktivitäten }\end{array}$ \\
\hline 3 & $\begin{array}{l}\text { Positionsverteidigungsstrategie in Verbindung mit } \\
\text { einer Abschöpfungsstrategie durch Rationalisierungs- } \\
\text { aktivitäten }\end{array}$ \\
\hline 4 & $\begin{array}{l}\text { Abschöpfungsstrategie durch Aktivitäten zur Erhaltung } \\
\text { der Cash Cow - Position }\end{array}$ \\
\hline 5 & $\begin{array}{l}\text { Abschöpfungsstrategie mit anschließender stufenweisen } \\
\text { Desinvestition durch } \\
\text { - Rationalisierungs- } \\
\text { - Spezialisierungsaktivitäten }\end{array}$ \\
\hline 6 & $\begin{array}{l}\text { Wachstums-/Investitionsstrategie durch } \\
\text { - Imageverbesserungs- } \\
\text { - Wettbewerberpositionssichernde Aktivitäten } \\
\text { mit langfristigem Ubergang zu einer Rationalisierungs- } \\
\text { strategie zur Erhohung der Ertragskraft }\end{array}$ \\
\hline 7 & $\begin{array}{l}\text { Nischenstrategie durch Spezialisierungsaktivitäten } \\
\text { in Verbindung mit einer Rationalisierungsstrategie } \\
\text { zur Erhöhung der Ertragskraft }\end{array}$ \\
\hline 8 & $\begin{array}{l}\text { Wachstums-/Investitionsstrategie durch } \\
\text { - Wettbewerbspositions- } \\
\text { - Imageverbessernde und } \\
\text { - Marktanteilssteigernde Aktivitäten }\end{array}$ \\
\hline 9 & $\begin{array}{l}\text { Abschöpfungsstrategie unter Ausnutzung der guten } \\
\text { Imageposition mit anschlleBender stufenweisen } \\
\text { Desinvestition }\end{array}$ \\
\hline 10 & $\begin{array}{l}\text { Reine Defensivstrategie, d.h. } \\
\text { - keine Investitionen zur Steigerung } \\
\text { des Marktanteils } \\
\text { - Rationalisierungsaktivitäten } \\
\text { Falls keine Verbesserung der Ertragssituation: } \\
\text { Desinvestition }\end{array}$ \\
\hline
\end{tabular}

Tab. 10: Endgültige Marketing-Basisstrategien auf der Basis eines sukzessiven Strategienvergleichs 
4.42 Kritische Würdigung und Ansatzpunkte zur Weiterentwicklung der Portfolio-Methode in Warenhäusern

Die Entwicklung von Marketing-Basisstrategien in Warenhäusern stellt im Gesamtprozeß der strategischen Marketingplanung das Bindeglied zwischen den globalen Rahmenentscheidungen (Ziele und Positionierung) und den detaillierten Marketing-Instrumentestrategien dar. In diesem Zusammenhang ist für die Portfolio-Methode zunächst positiv hervorzuheben, daß sich die auf ihrer Grundlage abgeleiteten Marketing-Basisstrategien innerhalb des durch die Rahmenentscheidung abgesteckten Bereichs bewegen. Dies wird zum einen durch die explizite Berücksichtigung relevanter Marketingziele wie z.B. Image, Marktanteil, Deckungsbeitrag in Form der Schlüsselfaktoren erreicht. Zum anderen berücksichtigen fast alle Portfolio-Varianten die an den Rahmenentscheidungen festgelegten Konkurrenzstrategien. Darüber hinaus wird die zunächst lediglich global und vage formulierte Positionierung gegenüber den Wettbewerbern durch den Konkurrenzbezug in den Portfolios im Hinblick auf die Waren- und Filialgruppen konkretisiert. Dabei weisen die entwickelten Warengruppenund Filialgruppenstrategien ihrerseits einen Globalitätsgrad auf, der einerseits so konkret ist, daß sie richtungsweisende Funktionen übernehmen können, aber andererseits so vage sind, daß die notwendigen Freiheitsgrade für die Entwicklung von Marketing-Instrumentalstrategien gewährleistet sind.

Der relativ hohe Globalitätsgrad kommt dadurch zustande, daß bei der Anwendung der Portfolio-Methode das strategische Problem bzw. der gesamte strategisch relevante situative Kontext auf einige wenige aussagekräftige Faktoren zurückgeführt wird. Diese Faktorenverdichtung trägt wesentlich zur Komplexitätsreduktion bei und erleichtert 
letzten Endes durch die Informationsverdichtung die Interpretation der strategischen Zusammenhänge und damit die Kommunikation innerhalb des Managements. Dies ist insbesondere für jene Warenhäuser wichtig, in denen allzu lange anstelle der konsequenten Marketingorientierung eine einkaufs- und einseitig konkurrenzorientierte Unternehmensführung verfolgt wurde.

Ein weiterer Vorteil der Portfolio-Methode wird deutlich, wenn über einen längeren Zeitraum hinweg kontinuierlich Portfolios erstellt werden. Diese lassen dann Positionsverschiebungen im Zeitablauf erkennen und zeigen, ob die entwickelte Strategie auch tatsächlich in der gewünschten Weise gewirkt hat. Hierdurch läßt sich die Portfolio-Methode zusätzlich als strategisches Kontrollinstrument nutzen. Hierzu ist es allerdings erforderlich, neben den Ist-Portfolios sog. Zielportfolios ${ }^{1} \mathrm{zu}$ erstellen. Sie zeigen die Positionen für Waren- und Filialgruppen auf, die in den nächsten z.B. fünf Jahren erreicht werden sollen. Weisen die in den nächsten Jahren erstellten Ist-Portfolios auf eine Abweichung vom gewünschten Entwicklungspfad hin, so können rechtzeitig Anpassungsmaßnahmen ergriffen werden. Diesen Grundgedanken verdeutlicht die Abbildung 23 exemplarisch für das MarktanteilsMarktwachstums-Portfolio.

Die Abbildung 23 beinhaltet die Positionierung von drei Warengruppen. Für die Jahre 1978 und 1979 liegen IstPositionierungen vor. Die langfristige Zielposition und die hierfür notwendige Marketingstrategie wurden 1978 festgelegt. Am Ende des Jahres 1979 stellt die Warenhausleitung das Ist-Portfolio auf und vergleicht die erreichten Positionen mit der Vorjahres- sowie der Zielposition für 1983. Dabei sind grundsätzlich 2 Fälle denkbar. (1) Die aktuelle Ist-Situation liegt genau in dem vorgezeichneten Positionsbereich und nähert sich der Zielposition (z.B. Warengruppe I und III). In diesem Fall ist eine Beibehaltung der gegenwärtigen Strategie angezeigt. (2) Weicht die aktuelle Ist-

1 Vgl. zu dem Gedanken, Zielportfolios zu erstellen, z.B. Abell, D.F., Hammond, J.S., a.a.O., S. 189 ff.; Day, G.S., a.a.O., S. 102, Hinterhuber, H.H., a.a.O., S. 122 ff.. 


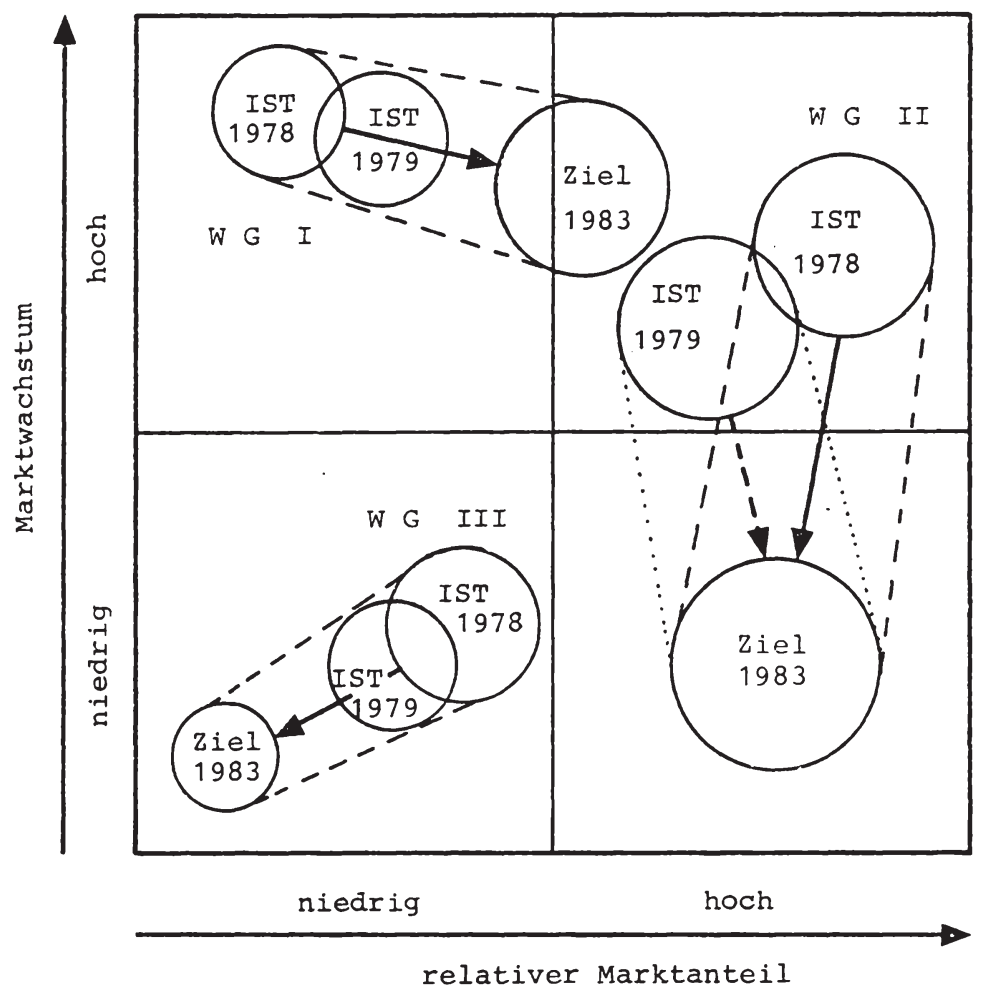

Legende: (1) Basisstrategien in 1978

Warengruppe I : Investitions-/Marktanteilssteigerungsstrategien

Warengruppe II : Investitions-/Marktanteilsverteidigungsstrategien

Warengruppe III : Desinvestitionsstrategie

(2) $\longrightarrow$ Wirkungsrichtung der im Jahr 1978 gewäh 1ten Basisstrategie

(3) $-\rightarrow$ Wirkungsrichtung der im Jahr 1979 gewählten Basisstratecie

(4) Modifizierte Basisstrategiein 1979 für Varengruppe II: Investitions-Marktanteilsrückgewinnungs-strategie

Abb. 23 : Zielorientierte Modifizierung von Basisstrategien 
Situation dagegen erheblich von der angestrebten Entwicklung $a b$ (z.B. Warengruppe II) und soll die Zielsetzung nach eingehender Analyse beibehalten werden, so muß die gewählte Strategie geändert werden. Hierzu sind detailierte Abweichungsanalysen notwendig, die die Ursache für den MiBerfolg der Strategie offenlegen und gleichzeitig die neue strategierichtung implizieren.

Im Hinblick auf die notwendige Konkurrenzorientierung der strategischen Marketingplanung von Warenhäusern ist es ferner angezeigt, zumindest für die unmittelbaren Warenhauskonkurrenten Ist-Portfolios zu erstellen, mit deren Hilfe sich ein unmittelbarer Positionsvergleich durchführen läßt. Bei einer komparativ-statischen Analyse über mehrere Jahre hinweg lassen sich darüber hinaus auf diese Weise Erkenntnisse über die strategischen Stoßrichtungen der Konkurrenten ermitteln, die die eigenen Planungen mit einbeziehen. und insbesondere bei der Evaluierung der eigenen Marketing-Basisstrategien berücksichtigt werden können. Weist z.B. ein Warenhauskonkurrent bei einer für ihn umsatzmäßig bedeutsamen Warengruppe ein vergleichsweise geringes Marketingpotential - gemessen z.B. am Kommunikationsaufwand oder Image - auf, so kann dies ein zentraler Ansatzpunkt für seine zukünftigen Marketing-Basisstrategien sein. Diese wahrscheinliche Strategie ist mit der eigenen Basisstrategie zu vergleichen. Falls diese Marketingstrategie dazu geeignet ist, der Strategie des Konkurrenten wirksam zu begegnen, ist sie beizubehalten. Andernfalls muß eine Modifikation erwogen werden. Hierbei sind alle zusätzlichen Informationen der Unternehmensund Konkurrenzanalyse zu berücksichtigen. Dies führt entweder $z u$ einer Neubewertung der betreffenden Warenbzw. Filialgruppe und damit zu einer Neupositionierung oder bei Bestätigung der Position zu einer modifizierten oder neuen Marketing-Basisstrategie im eigenen Unternehmen. Damit kann mit dieser Vorgehensweise die Richtigkeit der aus der Ist-Portfolio-Position entwickelten Strategie überprüft werden.

$1 \mathrm{Vgl}$. zu dem Gedanken, Konkurrenzportfolios zu erstellen, z.B. Hinterhuber, H.H., a.a.O., S. $97 \mathrm{ff}$. 
Neben den aufgezeigten Vorteilen der Portfolio-Methode müssen jedoch einige kritische Punkte angeführt werden. In diesem Zusammenhang ist zunächst ihre mangelnde theoretische Fundierung zu nennen. Die üblicherweise zugrundegelegten Schlüsselfaktoren sind bislang - mit Ausnahme des Marktwachstums-Marktanteils-Portfolios ${ }^{1}$ - weder in ihren Auswirkungen auf den Unternehmenserfolg noch in ihren wechselseitigen Zusammenhängen analysiert. Die vielfältigen Anwendungen in Herstellerunternehmungen beruhen fast ausnahmslos auf plausibilitätsüberlegungen. Wertet man den dort nachgewiesenen Erfolg beim Einsatz der Methode als empirische Bestätigung der Plausibilitätsüberlegungen, so steht diese für die reale Planungssituation im Handel noch aus, da bislang kaum Erfahrungen in der Anwendung der Portfoliomethode vorliegen. Insofern kann eine abschließende Beurteilung der Aussagekraft der oben diskutierten PortfolioVarianten nicht aus wenigen Anwendungsfällen, sondern erst nach einer Vielzahl zukünftiger Einsätze für handels- bzw. warenhausspezifische Planungsprobleme erfolgen. Solange diese aussteht, stellt jeder neue Anwendungsfall hohe Anforderungen an die Qualifikation, Flexibilität und Risikobereitschaft des Managements, das darüber hinaus stets eine kritische Distanz zur Portfolio-Methode bewahren muß.

Die Bedeutung des Managements bei der Anwendung der Portfolio-Methode kommt auch in der kritisch $\mathrm{zu}$ beurteilenden Notwendigkeit der mehrfach subjektiven Schätzung zum Ausdruck. Insbesondere bei synthetischen Portfolio-Varianten erfolgt eine subjektive Auswahl der als relevant anzusehenden Kriterien und ihres Bedeutungsgewichtes sowie eine subjektive Beurteilung der strategischen Geschäftseinheiten anhand jedes Kriteriums. Hierdurch wird die intersubjektive Nachprüfbarkeit der Positionierung der entwickelten Strategien zweifellos erschwert. Darüber hinaus fehlen bei

$1 \mathrm{Vgl}$. die ausführliche Beschreibung der theoretischen Grundlagen z.B. bei Roventa, P., a.a.O., S. $111 \mathrm{ff.}$ 
Anwendung von Punktbewertungsverfahren theoretisch exakte und begründbare Begrenzungen bzw. Trennlinien der einzelnen strategischen Felder der Portfolio-Matrix. Die sich aus dem Spektrum der Gesamtpunktwerte ergebende qualitative Aufteilung (z.B. hoch - mittel - niedrig) stellt eine grobe Heuristik dar ${ }^{1}$. Dieser Einwand wiegt deshalb schwer, weil in der "klassischen" Form der Portfolio-Analyse die Felder der Matrix mit spezifischen Strategieempfehlungen identisch sind (z.B. "Star" = investieren; "Cash Cow" = melken).

Insbesondere gegen die synthetischen Portfolio-Varianten ist vorzubringen, daß sie $z u$ einer unzulässigen Aufrechnung von Gewinnaussichten und Risiko führen können ${ }^{2}$. Durch die mehrfache Beurteilung und Verrechnung von strategischen Geschäftseinheiten hinsichtlich Gewinn (z.B. Rentabilität, Deckungsbeitrag) und Risikokriterien (z.B. Konkurrenzaktivitäten, staatliche Eingriffe) ist es denkbar, daß relevante Risikogesichtspunkte durch hohe Gewinnaussichten kompensiert werden. Gerade bei den hier zugrundeliegenden längeren Zeiträumen und der daraus resultierenden Unsicherheit der Datenbasis erscheint die Forderung nach einer expliziten Berücksichtigung des Risikos gerechtfertigt. Insofern liegt für'den konkreten Anwendungsfall zum einen die eindeutige Trennung der gewinn- und risikoorientierten Kriterien und ihre Darstellung in unterschiedlichen Portfolios nahe. Zum anderen sind in allen jenen Fällen, bei denen hohe Risiken sichtbar werden, spezifische Risikoanalysen - über die Portfolio-Analyse hinaus - durchzuführen. Durch die Erweiterungen bzw. Modifikationen ließe sich die Gefahr der ungenügenden Beachtung des Risikos zweife1los reduzieren.

1 Dieser Einwand trifft auch auf die analytischen Portfolios zu, denn die Felderabgrenzung erscheint auch dort relativ willkürlich.

2 Vgl. speziell zu diesem Einwand: Koch, H., Programmplanung, a.a.O., S. $151 \mathrm{f}$.. 
Weiterhin wird der Portfolio-Methode oftmals der Vorwurf gemacht, daß die entwickelten Basisstrategien unvollständig sind bzw. nicht alle strategisch relevanten Fragen beantworten. So wird z.B. bei einer Investitionsstrategie keine Aussage über das Investitionsvolumen getroffen ${ }^{1}$; es ist unklar, wann z.B. eine Warengruppe im Dog-Bereich aufgegeben werden soll oder mit welchen Marketing-Instrumenten eine Profilierung zu erreichen ist. Obgleich diese Einwände ihre Berechtigung haben, muß doch relativierend angemerkt werden, daB die Portfolio-Methode die strategischen Entscheidungen des Management $\mathrm{zu}$ unterstützen hat, dabei aber weder als Managementersatz noch als "Betriebsanleitung für strategien" ${ }^{2}$ fungieren kann. Insofern ist der Globalitätsgrad der Aussagen im Hinblick auf die $z u$ lösenden strategischen Problemstellungen als durchaus angemessen, $z$ u betrachten. 4

Entspricht der Globalitätsgrad der entwickelten Basisstrategien durchaus der hierarchischen Ebene der strategischen Geschäftseinheiten, so kann es sich andererseits im Rahmen der Warenhausplanung als sinnvoll erweisen, die hochaggregierte Warengruppen- und Filialgruppenebene zu verlassen und die Ebene der Artikelgruppe oder Einzelfilialen als strategische Geschäftseinheiten zu betrachten. Dies würde jedoch eine sehr viel gröBere Anzahl von strategischen Geschäftseinheiten implizieren, die im Rahmen der Planung kaum noch handhabbar wären ${ }^{3}$. Dabei hängt der

1 Vgl. zu diesem Kritikpunkt: Jünger, P., a.a.O., S. 109 .

2 Wittek, B.F., a.a.O., S. 141.

3 Bei Betrachtung der Artikelgruppen müssen ca. 80 strategische Geschäftseinheiten gebildet werden, bei Betrachtung der Einzelfilialen muß Karstadt $z$. B. über 150 strategische Geschäftseinheiten positionieren.

4 Dies gilt insbesondere, wenn man berücksichtigt, daß im Rahmen des strategischen Planungsprozesses eine stetige Konkretisierung der Strategien bis hin zu optimierenden Feinplanungsmethoden erfolgt (z.B. Mediaselektionsmodelle; Programmplanung und Regalplatzoptimierung mit der linearen Programmierung usw.). 
adäquate Aggregationsgrad der strategischen Geschäftseinheiten neben der strategischen Problemstellung auch von den im Anwendungsfall gemachten Erfahrungen ab. Erweisen sich einzelne strategische Geschäftseinheiten als unzweckmäßig, so können und müssen sie nach den neuen Erkenntnissen um- bzw. neudefiniert werden. Unter diesem Blickwinkel wirkt sich die Möglichkeit, im Planungsstadium relativ losgelöst von organisatorischen Zwängen handeln zu können, außerst positiv auf die Flexibilität und die frühzeitige Erfassung wichtiger Entwicklungen aus.

Diese Uberlegung berührt gleichzeitig das Problem der Informationsgrundlagen der Portfolio-Methode. Obgleich zur Positionierung der strategischen Geschäftseinheiten Informationen über die ausgewählten Schlüsselfaktoren genügen, gewinnt die Methode erst durch eine weitere informatorische Fundierung bzw. durch ihre Einordnung in ein umfassendes Analyse- und Informationssystem ihre vollständige Aussagekraft. Dies gilt insbesondere für die analytischen Portfolio-Varianten, da sie lediglich Informationen über zwei Schlüsselfaktoren direkt verarbeiten.

Insgesamt werden im dualen Denkmodell der strategischen Marketingplanung in Warenhäusern hochaggregierte Informationen über Waren- und Filialgruppen benötigt, die das planende Gremium (Vorstand mit Planungsstab) nur durch eine aktive Mitwirkung der nachgelagerten Instanzen gewinnen kann. Durch diese Mitwirkung im Vorstadium der strategischen Planung wird darüber hinaus frühzeitig die Basis für eine hinreichende Motivation der nachgelagerten Instanzen im Warenhaus gelegt ${ }^{1}$. Dieser Aspekt gewinnt insbesondere im Hinblick auf die vorhandenen "Spannungen" zwischen zentralen und dezentralen Stellen im Warenhaus seine Bedeutung.

1 Diese ist im Rahmen des gesamten Planungssystems durch adäquate organisatorische Regelungen sicherzustellen. 
Sieht man die Portfolio-Methode im Gesamtsystem der strategischen Marketingplanung von Warenhäusern, so vermag sie einen wesentlichen Beitrag zur Entwicklung eines geschlossenen Systems von globalen Marketingstrategien zu leisten. Sie stellt allerdings keinen Ersatz für differenzierte Planungsverfahren dar, die bei Teilproblemen eine detailliertere Strukturierung (z.B. Stärken-Schwächen-Analysen), eine abgesicherte Entscheidung (z.B. Wirtschaftlichkeitsrechnungen) oder eine optimierung bei vorgegebenen zielsetzungen (z.B. lineare Programmierung, Simulationsmodelle) sicherstellen. Insbesondere bei einer detaillierten Analyse von Einzelproblemen, z.B. bei der Ermittlung der rentabilitätsoptimalen Warengruppenstruktur im Sinne der Flächenvergaben, der Werbebudgetierung, der optimalen Verkäuferanzahl, der innerbetrieblichen Standortwahl, der Regalplatzzuweisung usw. können analytische Feinplanungsmethoden Platz greifen, die innerhalb des vorgegebenen Rahmens "gute" Lösungen hervorbringen. Ebensowenig kann die Portfolio-Methode die notwendige kreative Leistung bei der strategieentwicklung durch eine schematisierte Ableitung von Marketingstrategien ersetzen ${ }^{1}$.

1 Aus der Fülle der in der Literatur aufgezeigten Ansätze sei besonders verwiesen auf: Gümbel, R. et al. (Hrsg.), Unternehmensforschung im Handel, Rüschlikon-Zürich 1969; Tietz, B., Die Standort- und Geschäftsflächenplanung im Einzelhandel, Rüschlikon-Zürich 1969; Barth, K., Warenpräsentation, a.a.O., S. 35 ff.; Böcker, F., Sortimentsverbund, a.a.O., S. 179 ff.; Eckhardt, Zur Optimierung der Sonderangebotspolitik in Warenhandelsbetrieben - Eine empirische studie, in: ZfbF 30 (1978), S. 544-56o. 
Vielmehr erlaubt die Portfolio-Methode eine integrierte und komprimierte Darstellung der "Geschäftsfelder" eines Warenhauses. Sie trägt dazu bei, die komplexen Zusammenhänge im Warenhaus zwischen Wettbewerb, Kunde, Markt und eigenem Potential zu verstehen und fördert die Kommunikation zwischen den Planungsträgern. Auf fundierte weitere Analysen gestützt, zeigt sie die Auswirkungen von Marketing-Basisstrategien auf die relevanten Ziele von Warenhäusern und gibt durch die Analyse des strategischen Problems aus verschiedenen Perspektiven wesentliche Impulse im strategischen Planungs- und Entscheidungsprozeß. Sie stellt damit einerseits das notwendige Bindeglied und andererseits die richtungsweisende Voraussetzung zur Entwicklung konkreter Marketing-Instrumentalstrategien dar.

1 Ungeachtet dieser Schwachstellen findet die PortfolioMethode Eingang in weitere Bereiche. Vgl. hierzu z.B. Dewbold, G.D., Product Portfolio Analysis for U.S. Universities, in: Acron Business and Economic Review, Vol. 11, Nr. 1, Spring 1980, S. 39-45; Henzler, H., Neue Strategie ersetzt den Zufall, in: Manager Magazin, Heft 4/1979, S. 122-129; Schiers, J., Muß Bonn mehr planen, in: Manager Magazin 5/1980, S. 89-101; Simmerl, M., Portfolio-Selektion: Strategie gegen Zufallserfolge, in: ASW 21. Jg. (1978), Heft 5, S. 78-82. 
5. Konkretisierung der Basisstrategien durch MarketingInstrumentalstrategien

Aufgabe der Marketing-Instrumentalstrategien ist es, Aussagen über den Einsatz eines bestimmten Marketinginstrumentes, Instrumentebereiches oder des gesamten MarketingInstrumentariums mit einem bestimmten Aktivitätsniveau zu treffen. Hierzu bedarf es zunächst einer Charakterisierung, Abgrenzung und Systematisierung des Marketinginstrumentariums von Warenhäusern.

\subsection{Strukturierung der Marketing-Instrumentalstrategien}

\subsection{Systematisierung der Marketinginstrumente in Waren- häusern}

In der Literatur und Praxis finden sich zwahlreiche Kataloge von Marketinginstrumenten des Einzelhandels, die sich je nach Zwecksetzung primär in Anzahl und Gruppierung der Marketinginstrumente unterscheiden ${ }^{1}$.

Aufgrund der zielsetzung der Arbeit, realitätsbezogene Marketingstrategien auf der Basis von planungstheoretischen Erkenntnissen für Warenhäuser abzuleiten, sind an die Systematisierung der Marketinginstrumente folgende Anforderungen $\mathrm{zu}$ stellen:

- sie muß detailliert genug sein, um eine praxisgerechte, realistische Betrachtung $\mathrm{zu}$ ermöglichen

1 Diese werden hier nicht im Detail analysiert. Vgl. zur Darstellung von solchen Systematisierungen u.a. Ahlert, D. , Marketing, S.49ff.; Bearchell, C.A., a.a.O., S. 53 f.; Bohlen, W.H., a.a.O., S. 27 ff.; Falk, B.R., Wolf, J., Handelsbetriebslehre, a.a.O., S. 254; Hansen, U., Marketing, a.a.O., S. $144 \mathrm{ff}$; L Lazer, W., Kelley, E.J., a.a.0., S. $11 \mathrm{ff.;}$ Meffert, H., Marketing, a.a.o., S. 81 ff.; Oehme, W., Unternehmensführung im Handelsbetrieb, Wiesbaden, o.J., S. 44; Petermann, G., Marketinginstrumentarium im Handel, in: Handelsforschung heute. Festschrift zum 50-jährigen Bestehen der Forschungsstelle für den Handel, hrsg. v. der Forschungsstelle für den Handel, Berlin (FfH) e.V., Berlin 1979, S. 153166. 
- sie muß trotz der Detaillierung eindeutige Instrumentebereiche aufweisen

- sie muß eine Differenzierung in strategische und taktisch/operative Marketingentscheidungen erlauben und

- sie muß auf die spezifischen Probleme der Warenhäuser zugeschnitten sein.

Diese Anforderungen erfüllt die in der Abbildung 24 dargestellte Systematik. Sie teilt das Marketing-Instrumentarium von Warenhäusern in die Bereiche der Sortiments-, Distributions-, Kontrahierungs- und Kommunikationspolitik ein.

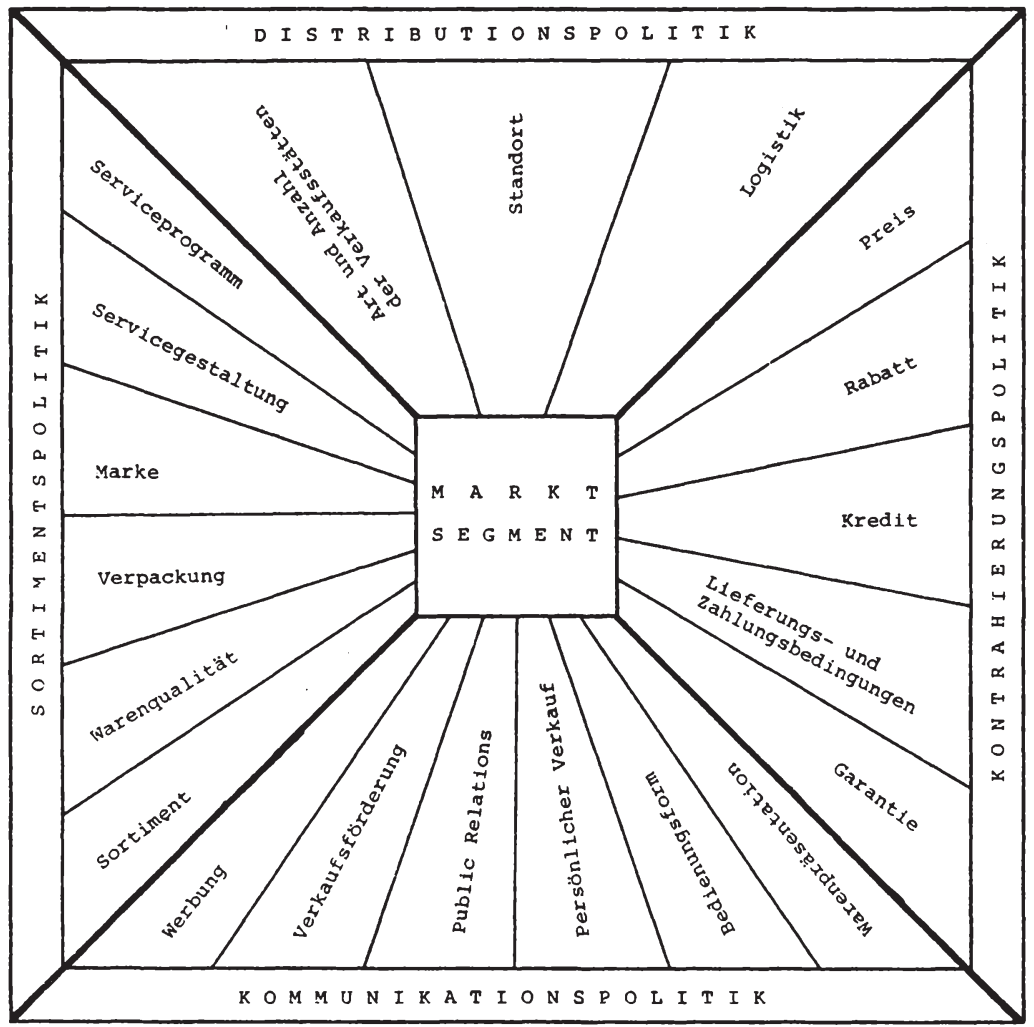

Abb. 24 : Systematik der Marketing-Instrumente in Warenhäusern 
5.12 Zusammenhang zwischen Marketing-Basisstrategien und Instrumentalstrategien

Diese Systematisierung der Marketing-Instrumente von Warenhäusern bildet den Ansatzpunkt für die Entwicklung der Marketing-Instrumentalstrategien. Dabei erhalten die hier systematisierten Marketing-Instrumente aufgrund der vorgelagerten strategischen Entscheidungen unterschiedliche Bedeutung. Differenziert man die Auswirkungen von Ziel- und Positionierungsentscheidungen sowie von Basisstrategien auf die Marketing-Instrumente nach dem Intensitätsgrad bzw. dem Grad der Prädisposition in mittelbare und unmittelbare Auswirkungen, so gilt vereinfacht, daß

- die Warengruppenstrategien sich unmittelbar auf die Sortimentspolitik, jedoch nur mittelbar auf die Kontrahierungs-, Distributions- und Kommunikationspolitik auswirken,

- die Filialgruppenstrategien sich unmittelbar auf die Distributionspolitik, jedoch nur mittelbar auf die sortiments- , Kontrahierungs- und Kommunikationspolitik auswirken

- und daß die Ziel- und Positionierungsentscheidungen sich unmittelbar auf alle Marketinginstrumente-Bereiche auswirken $^{1}$.

Hieraus läßt sich ein zweistufiges Vorgehen entwickeln (vgl. Abbildung 25 ). In der ersten Stufe werden die aus den isolierten Warengruppen- und Filialgruppenstrategien resultierenden unmittelbaren Marketing-Instrumental-

1 Dabei ist aufgrund der generellen Interdependenz der Marketing-Instrumente selbstverständlich eine exakte und eindeutige Trennung zwischen unmittelbaren und mittelbaren Auswirkungen schwierig durchzuführen, zumal $z$.B. die primär durch die Warengruppenstrategie bestimmten produktpolitischen Strategien alle anderen Instrumente-Bereiche stark beeinflussen. Aufgrund der nicht realisierbaren Simultanplanung erscheint trotz dieses Mangels eine sukzessive Vorgehensweise, die auf einer Hierarchie der Auswirkungen auf die MarketinginstrumenteStrategie aufbaut, zur Verdeutlichung der Problemstruktur sinnvoll. 
strategien der Sortiments- und Distributionspolitik abgeleitet, wobei die übrigen Rahmenentscheidungen als Nebenbedingungen beachtet werden müssen. Dabei erfolgt durch mehrfache Rückkoppelung einerseits eine Abstimmung mit den Basisstrategien sowie andererseits eine Koordination von sortiments- und distributionspolitischen strategien. Damit ist die Basis für die zweite Stufe, die Ableitung der übrigen Marketing-Instrumentalstrategien geschaffen. Diese sind durch die vorgelagerten Entscheidungen vorgeprägt und müssen auf kreativ-deduktive Weise abgeleitet werden. 1

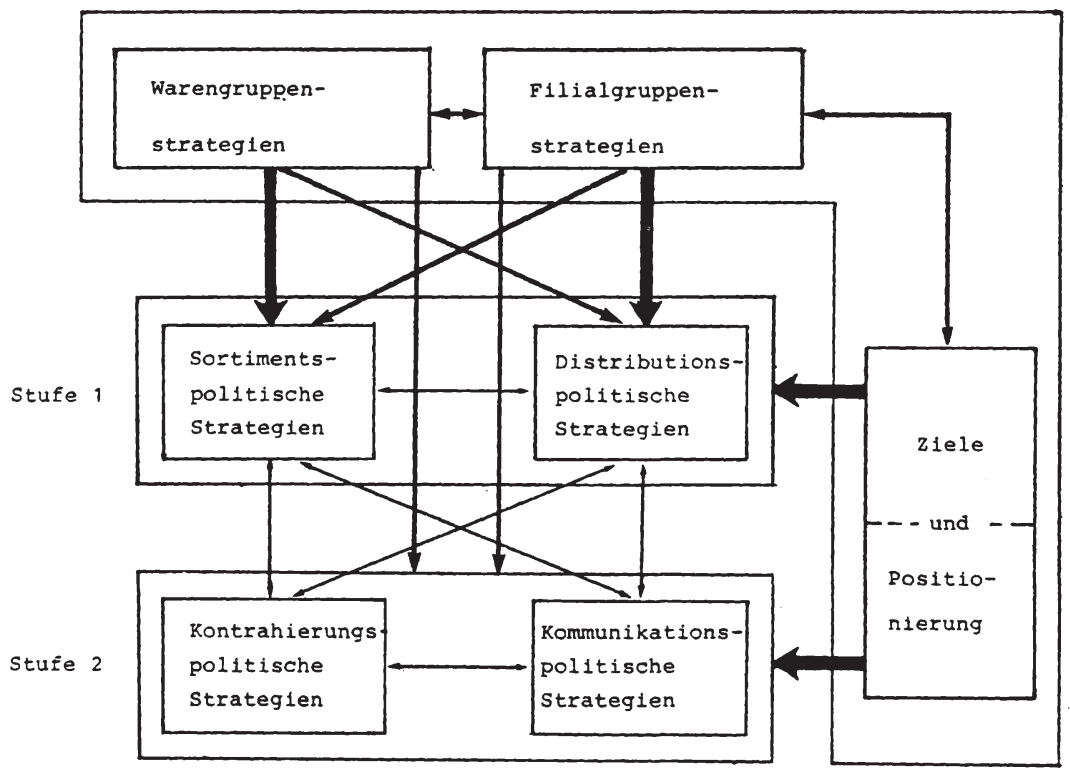

Abb. 25 : Mehrstufig-heuristische Vorgehensweise zur Ableitung von Marketing-Instrumentalstrategien

1 Hierbei handelt es sich generell um einen systematischgeistigen Prozeß, der sich einer vollständigen formalen Strukturierung - auf dieser globalen Ebene - weitgehend entzieht. (Vgl. hierzu z.B. Angehrn, O., System des Marketing, Bern und Stuttgart 1973, S. 237). Lediglich in Teilbereichen (auf disaggregierter Ebene) sind formale Planungsmethoden einzusetzen, die unter bestimmten Prämissen "optimale" Lösungen generieren (z.B. Preisstrategien, Werbebudgetierung, Streuplanung). 
Marketing-Instrumentalstrategien können im Wege der zweistufigen Vorgehensweise aufgrund der engen Verzahnung der Marketing-Basisstrategien mit den sortiments- und distributionspolitischen Instrumentalstrategien sinnvollerweise nur für klar umrissene Marketing-Basisstrategien konkret formuliert werden. Betrachtet man dementsprechend die mit Hilfe der Portfolio-Methode entwickelten Waren- und Filialgruppenstrategien, so ergibt sich für ihre Gesamtheit ein breites Spektrum von Strategiealternativen, das von einem stufenweisen Abbau der Warengruppen bzw. Filialgruppen über eine Konsolidierungsstrategie bis hin zu einer Marktanteilssteigerung bzw. Expansionsstrategie reicht. Diese Strategievielfalt zwingt zu einer beispielhaften, die strukturierung und Handhabung des Planungsproblems verdeutlichenden Darstellung. Deshalb werden aus der vielzahl der abgeleiteten Basisstrategien einige wenige herausgegriffen und an ihnen die Vorgehensweise exemplarisch verdeutlicht.

Unter dieser Perspektive bietet es sich im Hinblick auf eine differenzierte Darstellung der Marketing-Instrumentalstrategien an, zunächst die Extrema auf dem "Strategienkontinuum", d.h. die Erhaltungs- bzw. Schrumpfungsstrategie und die Expansionsstrategie, auszuwählen.

Dabei zielen Erhaltungs-bzw. Schrumpfungsstrategien primär auf eine Erhaltung des Status quo ab. Sie nehmen eine Verschlechterung des Erfüllungsgrades quantitativer Marketingziele (wie z.B. Umsatz, Marktanteil, Verkaufsfläche) in $\operatorname{Kauf}^{1}$. Dagegen streben Expansionsstrategien

1 Hier sind z.B. die Strategietypen "Desinvestition", "stufenweiser Abbau" und "Abschöpfung" einzuordnen. 
danach, diese ziele zu steigern ${ }^{1}$. Neben diesen beiden "extremen" Strategietypen werden im folgenden weiterhin Marketing-Instrumentalstrategien für einen Strategietyp in der Mitte des strategiekontinuums formuliert. Diese mittlere Position nehmen die Konsolidierungs strategien ein, denen darüber hinaus in der aktuellen Situation der Warenhäuser zentrale Bedeutung zukommt. Sie sind darauf ausgerichtet, entweder quantitative interne Ziele (z.B. ROI, DB, Gewinn, Rationalisierung) oder qualitative externe Ziele (z.B. Image, Profilierung, Kompetenz) ${ }^{2}$ zu verbessern.

Im Hinblick auf den durch die Positionierungsentscheidung gelegten Rahmen gilt i.d.R.für Marketing-Instrumentalstrategien die Richtlinie einer Profilierungsstrategie gegenüber der Konkurrenz und einer gezielten Ansprache der Käuferschaft der gehobenen Mitte.

Anhand der ausgewählten drei Strategietypen werden im folgenden zunächst sortiments- und distributionspolitischen Marketingstrategien von Warenhäusern beispielhaft dargestellt und diskutiert ${ }^{3}$.

\subsection{Entwicklung sortimentspolitischer Marketingstrate- gien von Warenhäusern}

Die Sortimentspolitik, der Kern des Marketing von Warenhäusern, beinhaltet einerseits als zentrales Element die

1 Hierzu zählen z.B. die im Rahmen der Warengruppenwahl abgeleiteten Basisstrategien "Positionsverbesserung", "Marktanteilsverbesserung", "Forcieren", "Investition".

2 Hier sind z.B. Basisstrategien wie "Positionspflege" oder "Rationalisierung" einzuordnen.

3 Die aufgezeigten Strategien dürfen dabei keinesfalls als konkrete Empfehlungen für die Praxis interpretiert werden. Sie verdeutlichen nur beispielhaft den Gedankengang. 
Zusammensetzung eines zielgruppenspezifisch attraktiven Sortiments ${ }^{1}$ und andererseits alle Entscheidungen über die Qualitätslage, die Markenpolitik und die Servicepolitik.

\subsection{Sortimentsstrategien in Warenhäusern}

Im Rahmen von Sortimentsstrategien ${ }^{2}$ wird die art- und mengenmäßige Zusammensetzung des Warenhaus-Sortiments festgelegt ${ }^{3}$. Ihre konkrete Ausgestaltung ist durch die jeweils festgelegte Warengruppenstrategie vorausbestimut.

Dabei wird bei expansionsorientierten Basisstrategien eine stetige Ausweitung des Sortiments sowohl im Hinblick auf die Sortimentstiefe als auch -breite angestrebt. Dies impliziert im ersten Fall den starken Ausbau bestehender Warengruppen, d.h. die Diversifikation ${ }^{5}$ in neue Branchen.

1 Vgl. z.B. Lazer, W., Kelley, E.J., a.a.O., S. 11; Petermann, G., Instrumentarium, a.a.O., S. 158.

2 Vgl. zur Sortimentspolitik allgemein z.B. Buskirk, R.H., Buskirk, B.D., a.a.O., S. 210 ff.; Davidson, W.R. et al., a.a.O., S. $312 \mathrm{ff.;}$ Falk. B.R., Wolf. J., Handelsbetriebslehre, a.a.O., S. $260 \mathrm{ff.;}$ Gümbel, R., a.a.O., S. 53 ff.; Hansen, U., Marketing 1, S. $193 \mathrm{ff.;}$ Mangold, H.G., a.a.O., S. 65 ff..

3 Vgl. Meffert, H., Marketing, a.a.O., s. 371.

4 Dabei wird die Forcierung aller Warengruppen durch eine ständige Aktualisierung, Umgestaltung und Ausweitung auf der disaggregierten Artikelgruppen- oder Artikelebene realisiert und ist somit der operativen Marketingplanung zuzurechnen.

5 In Einzelhandelsunternehmungen wird üblicherweise bereits dann von Diversifikationsstrategien gesprochen, wenn neue Warengruppen in das Sortiment aufgenommen werden. Vgl. zur Begriffssystematik: Borschberg, E., Diversifikations-Strategien, a.a.0., s. $83 \mathrm{ff} . \mathrm{Zu}$ einer Analyse aus Herstellersicht vgl. z.B. Meffert, H., Die Durchsetzung von Innovationen in der Unternehmung und im Markt, in: ZfB 46 (1976), S. 77-100. 
Beide Strategieausprägungen müssen eine Reihe von Anforderungen erfüllen, die sich an den Marketingzielen (Umsatz, Warenrohertrag, Lagerkosten, Image usw.), der Zielgruppenadäquanz und der Profilierung gegenüber der Konkurrenz orientieren.

In der Praxis wurde diese Ausbaustrategie von den Warenhäusern in den vergangenen Jahren primär verfolgt. Dabei führte der stetige Ausbau der bestehenden Warengruppen zu einer "Sortimentsaufblähung", die heute vielfach als Faktor für die schlechte Ertragslage der Warenhäuser verantwortlich gemacht wird ${ }^{1}$.

Unter dieser Perspektive rückt die Konsolidierungsstrategie in den Vordergrund, die in der Regel eine Umschichtung des Sortiments impliziert. Dementsprechend werden die sortimentspolitischen überlegungen neben den marktorientierten zielsetzungen in hohem Maße von internen ertragsbetonten Zielsetzungen gesteuert. Die Umschichtung des Sortiments wird dabei idealtypischerweise so vollzogen, daß ertragsstarke Warengruppen wie z.B. DOB, Haka, Persönlicher Bedarf sowie Freizeitbedarf ausgebaut und ertragsschwache Warengruppen wie z.B. Schuhe, Kleintextilien sowie Lebensmittel reduziert werden ${ }^{2}$. Hierzu bedarf es einer genauen Analyse und Prognose der relevanten Entwicklungen (z.B. Marktanteile, Markttrends), um zukünftige Verschiebungen antizipieren zu können. Zum anderen müssen die

1 Vgl. Krüger, H., a.a.O., S. 63 f..

2 Die Umschichtungsstrategie darf nicht im Sinne einer "wachstumsverhindernden" Strategie interpretiert werden. Allerdings erfolgt durch die Berücksichtigung von ertragsorientierten Kriterien bei der Sortimentsgestaltung eine Abkehr vom "reinen" Wachstumsdenken. Aus diesem Grunde spricht man in diesem Zusammenhang oft vom "qualitativen" Wachstum. 
zu reduzierenden Warengruppen im Hinblick auf ihre Bedeutung für die Gesamtattraktivität des Warenhauses analysiert werden ${ }^{1}$, was im Einzelfall zur Revidierung der Ausgangsentscheidung führen kann.

Entscheidet sich die Warenhausleitung für eine Erhaltungsbzw. Schrumpfungsstrategie, so ist das Sortiment im Hinblick auf eliminierungsverdächtige Bereiche zu untersuchen. Zu ihnen gehören primär jene Warengruppen, die entweder in gewinnorientierter Hinsicht oder unter dem Blickwinkel marktlicher Akzeptanz und Profilierung schlecht beurteilt werden.

Vor einer endgültigen Eliminierungsentscheidung ist jedoch zu überprüfen, ob der durchschnittliche Käufer die betroffene Warengruppe im Warenhaussortiment erwartet bzw. nur dann das Warenhaus besucht, wenn die betreffende Warengruppe auch geführt wird. In diesem Fall müssen die negativen Auswirkungen im Sinne von Image- oder Attraktivitätsverlust als Nebenbedingung der Entscheidung berücksichtigt werden. Eine weitere Restriktion kann sich darüber hinaus auch aus der Sortimentsstrategie der Konkurrenten ergeben, wenn diese eine Expansionsstrategie verfolgen.

Aufgrund dieser Gefahren, die aus dem fehlenden strategischen "Gleichschritt" der Warenhauskonzerne resultieren, bildet die strategie der Sortimentsreduktion heute noch die Ausnahme. Darüber hinaus ist die Wachstumsorientierung der Warenhäuser noch keineswegs gebrochen, wie die durchaus optimistischen Prognosen bzw. verkündeten zielsetzungen verschiedener Warenhausvorstände zeigen ${ }^{2}$.

1 So galt z.B. bislang die Reduzierung des Lebensmittelbereiches aufgrund der hohen akquisitorischen Wirkung als nicht durchführbar. Inwiefern durch den Schritt von Horten, die gesamte Abteilung Edeka zu überlassen, ein Meinungsumschwung eingeleitet wird, bleibt abzuwarten.

2 Vgl. z.B. O.V., Der Kaufhof will Vorwärtsstrategie beibehalten, in: FAZ, Nr. 116, 20. Mai 1980, S. 13; 0.V., Der "neue Horten" will Freizeitspezialist sein, in: FAZ, Nr. 124, 30. Mai 1980, S. 17. 
5.22 Marken-, Qualitäts- und Servicestrategien in Warenhäusern

Marken-, Qualitäts- und Servicestrategien nehmen im Rahmen des Warenhausmarketing die Funktion flankierender Strategien zur Unterstützung der Sortimentsstrategie ein.

Im Rahmen der Markenstrategie ${ }^{1}$ ist zum einen über den Anteil der markierten Ware im Gesamtsortiment und zum anderen über die Eigenmarkenpolitik zu entscheiden. Unter dem Gesichtspunkt, daß in jüngster Zeit die markierte Ware wieder positiv in das Bewußtsein der Verbraucher rückte ${ }^{2}$, ist ihr auch im Warenhaussortiment große Bedeutung zuzumessen. Denn durch attraktive Marken können die Kundenfrequenz, die Kundentreue und damit der Umsatz gesteigert werden. Grundsätzlich können diese Ziele sowohl mit Hersteller- als auch Handelsmarken ${ }^{3}$ erreicht werden.

$1 \mathrm{Vgl}$. zum Problemkreis der Markenpolitik im Handel

z.B. Gross, H., Markenpolitik, in: Nieschlag, R., Eckardstein, D., Der Filialbetrieb als System. Das CorneliusStüssgen-Model1, Köln 1972, S. 211-230; Hansen, U., Marketing 1, a.a.O., S. 249 ff.; Mangold, H.G., a.a.O., S. 102 .

2 Vgl. hierzu neuere Untersuchungen, z.B. Meffert, H., Der Markenartikel und seine Bedeutung für den Verbraucher, in: Gruner + Jahr Schriftenreihe, Band 24, Hamburg 1979, insbesondere S. $23 \mathrm{ff}$. (im folgenden zitiert als: Meffert, H., Markenartikel).

3 Das Problem der Herstellermarke versus Handelsmarke ist in der Literatur ausführlich diskutiert. Vgl. z.B. Angehrn, O., Handelsmarken und Herstellermarken im Wettbewerb, Stuttgart 1969; Barth, K., Rentable Sortimente im Handel. Zufall oder Ergebnis operationaler Entscheidungshilfen? in: Sonderhefte der Mitteilungen des Instituts für Handelsforschung an der Universität $z u$ Köln, hrsg. v. E. Sundhoff, Nr. 26, Köln 1980, S. 14 ff. (im folgenden zitiert als Barth, K., Sortimente); Gross, H., a.a.O., S. 211 ff.; Mangold, H. G., a.a.O., S. 102 ff.; Schenk, O., Hersteller- und Handelsmarken im Wandel ihrer gegenseitigen Beziehungen, in: Der Markenartikel 2/1970, S. 39-47; Steckenborn, I., Handelsmarken wachsen im Windschatten, in: Rationeller Handel 3/1976, S. 48-52. In jüngster Zeit wird die Diskussion durch das Aufkommen sog. "no-name-Produkte" wieder verstärkt geführt. Vgl. z.B. Raithel, H., Innovation ohne Namen, in: MM 2/1979, S. 52-59; Theuer, G., Brisante Frage an den Handel: Namenlose Billigsortimente oder Up-Trading? in: Markenartikel 1/1980, s. $20 \mathrm{ff.}$ 
Dabei ist $z u$ berücksichtigen, daß bekannte Herstellermarken das Warenhaussortiment profilieren und daß die mit ihnen verbundenen Vorstellungen einer hohen Qualität das Warenhaussortiment aufwerten. Somit kann das Warenhaus durch die strategisch ausgerichtete Streuung der Herstellermarken über das ganze Sortiment als Einkaufsstätte am guten Produktimage partizipieren. Gerade in der aktuellen Situation der Profilierungsversuche hat die bewußte Orientierung der Warenhäuser an Herstellermarken durchaus ihre Berechtigung.

Andererseits ist es für Handelsunternehmungen unter ökonomischen Gesichtspunkten oftmals sinnvoll, sich nicht ausschließlich auf viele individuelle Produktmarken zu stützen, sondern Eigenmarken- bzw. Eigenmarkenfamilien zu schaffen, in denen Irradiationseffekte ${ }^{1}$ ausgenutzt werden können.

Markiert ein Warenhaus Artikel verschiedener Eigenschaften mit einer einzigen Marke (z.B. Kaufhofmarke "Elite"), so hat diese Marke den Charakter eines Gütesiegels. Nur so lassen sich bei Wahrung der Markenpersönlichkeit völlig unterschiedliche Artikel mit einem Namen (als Markierungszeichen) benennen. Ebenso wie die Herstellermarke dient auch die Eigenmarke als Orientierungs- und Einkaufshilfe für den Käufer ${ }^{2}$. Dementsprechend kommen Eigenmarken insbesondere in Sortimentsbereichen mit einem hohen Anteil anonymer Ware bzw. in jenen Bereichen, in denen die Herstellermarken einen entsprechenden spielraum offenlassen, zum Einsatz. Um in den Augen der Konsumenten mit

1 Hierunter wird die Übertragung von Images von einem Produkt auf das andere verstanden. Vgl. Hansen, U., Marketing, Bd. 1, a.a.O., S. 259.

2 Als Funktionen des Markenartikels werden üblicherweise herstellerbezogene (z.B. Profilierung, Imagebildung), handelsbezogene (z.B. Geschäftsstättenprofilierung, Kundensicherung) und verbraucherbezogene Funktionen (z.B. Orientierung, Information, Identifikation, Entscheidungshilfe) bezeichnet. Vgl. hierzu Meffert, H., Markenartikel, a.a.O., S. $29 \mathrm{ff}$. 
der Eigenmarke keine Marke "zweiter Klasse" zu schaffen, sind diese vornehmlich im mittleren bis hohen Qualitätsniveau anzusiedeln.

Im Hinblick auf das Verhältnis von Hersteller- zu Eigenmarken ist im Sinne einer kontinuierlichen Profilierung eine "Mischstrategie" anzustreben, die neben bekannten und beliebten Markenartikeln ein breites Angebot an Eigenmarken vorsieht. Dem Käufer gegenüber wird damit eine "doppelte" Qualitätskompetenz demonstriert, die die Chancen auf eine Honorierung deutlich erhöht. Dabei sind dem Anteil der Eigenmarken Grenzen zu setzen, damit sie den Charakter des Besonderen bewahren und das Gütesiegel nicht als bloße Herkunftsbezeichnung fungiert ${ }^{1}$.

Das relativ stark ausgeprägte Qualitätsempfinden der Warenhauszielgruppe ${ }^{2}$ muß ebenso wie in die Markenpolitik auch in die Qualitätslagen-Strategie eingehen. In dieser Hinsicht kommt der technischen Qualität der Waren eine wesentliche profilierende Funktion $\mathrm{zu}^{3}$. Dementsprechend sind die Artikel des unteren Qualitätsbereichs den Kleinpreisgeschäften zu überlassen.

Die hohen qualitativen Ansprüche im Rahmen der Sortimentsstrategie bedingen ein gleichwertiges Serviceprogramm ${ }^{4}$.

1 Eine Quantifizierung dieser Aussage ist schwierig. Orientiert man sich an der Warenhauspraxis, so kann man als Beispiel die erfolgreiche Kaufhof-Eigenmarke "Elite" heranziehen. Sie erreichte 1975 einen Anteil von ca. 10\% am gesamten Kaufhof-Umsatz.

2 Vgl. hierzu auch Kapitel 3.2.

$3 \mathrm{Vgl}$. den guten Uberblick zum Problemkreis der Produktqualität bei Hansen, U., Marketing 1, a.a.O., S. 249 ff. sowie die dort angegebene Literatur.

$4 \mathrm{Vgl}$. zum Problemkreis der Servicepolitik im Handel die grundlegenden Ausführungen von Gerstung, F., Die Servicefunktion als Instrument des Handelsmarketing, Göttingen 1979. Vgl. zum Úberblick weiterhin Bolen, W.H., a.a.O., S. 411 ff.; Falk, B.R., Wolf, J., Handelsbetriebslehre, a.a.O., S. $273 \mathrm{ff.;}$ Hansen, U. Marketing 2, a.a.O., S. 213 ff.; Mangold, H.G., a.a.O., S. 178 ff.; vgl. zu diesem Problemkreis im Herstellerbereich z.B. Lo, L., Prognoseinformationen für kundendienstpolitische Entscheidungen, dargestellt an Beispielen des Fotomarktes, Diss. Münster 1979 . 
Hierbei ist die art- und mengenmäßige Zusammensetzung des Serviceprogramms festzulegen.

Im Hinblick auf die gewählten Basisstrategien impliziert eine expansionsorientierte Strategie die Erweiterung des Serviceprogramms. Ergeben sich hierdurch die gewünschten Imageverbesserungen beim Käufer, so kann sich dies durchaus in einem höheren quantitativen $Z$ ielerreichungsgrad (z.B. Umsatz, Kauffrequenz usw.) niederschlagen. Gerade deshalb werden die Warenhauskonkurrenten einen spürbaren "Profilierungsvorsprung" im Servicebereich jedoch schnell zu kompensieren suchen.

Bei einer übergeordneten Konsolidierungsstrategie muß die Warenhausleitung einen KompromiB zwischen internen ertragsorientierten und externen profilierungsorientierten Zielsetzungen finden. Denn einerseits verbietet die starke Kostenbelastung einen weiteren Ausbau des Serviceprogramm, andererseits aber erfordert die Zielsetzung der Profilierung gegenüber den Konkurrenten ein gewisses Maß an eigenständigen Aktivitäten in diesem Bereich. Deshalb muB unter dem Primat der Ertragsorientierung einer vorsichtigen Erweiterung des Serviceprogramms der Vorzug gegeben werden. Ihr Ziel muß es sein, einerseits den Konkurrenzvorsprung bei normalerweise erwarteten Servicearten für den Käufer nicht offenkundig werden $\mathrm{zu}$ lassen und andererseits durch punktuelle Eigenkonzepte Profil zu gewinnen. Dabei ist auch zu überprüfen, ob Serviceleistungen auf externe Organisationen übertragen werden können, bei denen das Warenhaus nur noch vermittelnd tätig wird.

Im Rahmen einer Erhaltuncsstrategie wird das Serviceprogramm ebenfalls auf dem gegenwärtigẹn Niveau gehalten. 
Allerdings muß bei dieser Strategie mit negativen Auswirkungen im Hinblick auf die Akzeptanz beim Kunden (z.B. Image, Kaufhäufigkeit) gerechnet werden, da sich die Konkurrenten diesem passiven Verhalten kaum anschließen werden ${ }^{1}$. Deshalb wird diese Strategie kaum realisiert werden.

Neben der Sortimentspolitik ist in der ersten Planungsstufe der Konkretisierung der Basisstrategien durch Marketing-Instrumentalstrategien die Distributionspolitik festzulegen. Hierbei dienen in erster Linie die Filialgruppenstrategien als Planungsbasis.

\subsection{Entwicklung distributionspolitischer Marketingstrate- gien von Warenhäusern}

$\mathrm{Zu}$ den strategischen Entscheidungen der Distributionspolitik zählen die Standortwahl sowie die Festlegung der Art und Anzahl der Verkaufsstätten ${ }^{2}$. Da beide Entscheidungstat-

1 Dies belegt auch die reale warenhaussituation. Sie zeigt, daß jeder Warenhauskonzern versucht, den anderen durch immer neue Serviceleistungen $z u$ überbieten. Vgl. hierzu die beispielhafte Aufzählung bei: Heiner, H., Bis zu 60 Dienstleistungsangebote für den Warenhauskonzern, in: $\mathrm{BdW}, \mathrm{Nr} .77,31.3 .1979$, S. 5.

2 Bei der Diskussion der distributionspolitischen Strategien wird auf die Analyse des Marketinginstrumentes Logistik verzichtet. Die Logistik ist zweifellos strategischen Entscheidungen zuzuordnen, jedoch sind die Problemstellungen aufgrund der bislang erreichten Größe der Warenhäuser für die zugrunde liegenden Strategietypen nahezu identisch. Insofern soll hier der Hinweis auf die im Zuge der aktuell verschlechterten Ertragslage der Warenhäuser verstärkte Notwendigkeit der rationellen logistischen Aktivitäten genügen. Einen anschaulichen Überblick am Beispiel Kaufhof bietet: Bruckhaus, M., Organisation der Kaufhof AG, in: ZO 3/1979, S. 123-132, hier S. 128 ff.; vgl. zur allgemeinen Diskussion der Logistik als Marketinginstrument im Einzelhandel z. B.: Bolen, W. H., a.a.O., S. 225 ff., Buskirk, R. H., Buskirk, B. D., Retailing, New York usw. 1979, S.268 ff.. 
bestände sehr langfristig Mittel binden und im Hinblick auf ihre weitreichenden Konsequenzen bezüglich der Zielerreichung nur schwierig revidierbar sind, kommt ihnen besondere Bedeutung $\mathrm{zu}^{1}$.

\subsection{Standortbezogene Strategien}

Bei der Entwicklung von standortbezogenen Strategien sind neben den aus den vorgelagerten Planungsstufen resultierenden Entscheidungsrestriktionen eine Reihe von Situationsfaktoren $\mathrm{zu}$ beachten, die die Qualität eines Standorts charakterisieren. Hierzu zählen primär

- kundenbezogene Faktoren, wie z.B. Passantenfrequenz, Kaufkraftpotential, Einkaufsverhalten

- konkurrenzbezogene Faktoren wie z.B. Anzahl, Größe, Betriebstyp, Sortimentsschwerpunkte

- lagebezogene Faktoren wie z.B. Verkehrssituation ${ }^{2}$, Parkmöglichkeiten, Entfernung zum Ortskern

- finanzielle Faktoren wie z.B. Miete, Grundstückskosten, Lohnniveau, örtliche Steuersätze.

Gegenstand standortbezogener Strategien sind zum einen

1 Teilweise werden sie daher als "konstitutive Entscheidungen" bezeichnet. Vgl. hierzu Falk, B.R., Wolf, J., a.a.O., S. $238 \mathrm{ff}$..

2 Vgl. hierzu auch die aktuelle Diskussion über die Bedeutung von Fußgängerzonen für den Einzelhandel, z.B.: Spannagel, R., Strukturwandel im Einzelhandel durch Fußgängerzonen, in: FfH-Mitteilungen, Neue Folge, Januar 1979, S. 1-6. 
die Standortneuwahl ${ }^{1}$ sowie die Festlegung der Filialgröße und zum anderen die Anpassung vorhandener standorte.

Bei expansionsorientierten Basisstrategien bieten sich zwei alternative Strategien an: der Ausbau bestehender Filialen oder die Erhöhung ihrer Anzahl durch Neueröffnungen. Im Hinblick auf die Ausbaustrategie verhindert allerdings die Citylage der meisten Warenhaus-Filialen durch restriktive städtebauliche Verordnungen eine spürbare Vergrößerung der Verkaufsfläche. Von daher ist die strategie der Neugründung von Filialen vordringlich zu betrachten, womit unmittelbar die Frage nach der Standortneuwahl aufgeworfen ist.

Für die Strategie der Standortneuwahl gelten jedoch gleichermaßen die genannten externen Restriktionen und schränken sie in ihrer Realisierungsmöglichkeit wesentlich ein. Denn in der realen Situation sind die normalerweise von Warenhäusern bevorzugten Citylagen in mittleren bis großen städten bereits vergeben. Dementsprechend

1 Die Wahl des Standortes wird üblicherweise den strategischen Entscheidungen des Handels zugerechnet. Gängigerweise wird die Entscheidung prozeBorientiert in mehreren Phasen dargestellt: von der Problemerkennung ïber die Festlegung potentieller Standorte, ihre Bewertung anhand spezifischer Kriterien bis zur Auswahl des endgültigen Standortes. Als Kernproblem der Standortwahl kann die orientierung an bestimmten Standortbedingungen interpretiert werden. Dies umfaßt insbesondere die Auswahl relevanter $\mathrm{zu}$ erhebender Informationen sowie ihre Gewichtung. $\mathrm{Zu}$ diesem Problemkreis finden sich zahlreiche Beiträge sowohl aus wissenschaftlicher als auch praktischer Sicht. Vgl. z. B. Berman, B., Evans, J.R., a.a.O., S. 191 ff.; Falk, B.R., Wolf, J.R., Handelsbetriebslehre, a.a.O., S.216 ff.; Hansen, U., Einzelhandel, Band I, a.a.O., S. $170 \mathrm{ff}$.. Nauer, E., Standortmodell und Standortpolitik im Einzelhandel, Bern/Stuttgart 1970; Tietz, B., Die Standort- und Geschäftsflächenplanung im Einzelhandel, Rüschlikon - Zürich 1969; Wotzka, P., Standortwahl im Einzelhandel, Hamburg 1970. 
können Warenhäuser neue Standorte meist nur durch Aufkauf anderer Unternehmen oder durch Ausweichen in weniger attraktive Geschäftszonen (Randlage, Vorortlage) bzw. in kleinere Orte gewinnen ${ }^{1}$. Insbesondere die Ausweichstrategie impliziert eine Anpassung der Standortkriterien auf ein niedrigeres Anspruchsniveau.

Als Ausweg aus dieser problematischen Lage werden in jüngster Zeit insbesondere verschiedene Strategievarianten diskutiert, die sich unter dem Begriff der "Branching-outStrategie" zusammenfassen lassen ${ }^{2}$. Dieser Strategietyp beinhaltet insofern mehrere Varianten, als er u.a. die Möglichkeit bietet

- Abteilungen bzw. Warengruppen völlig aus der bestehenden Filiale auszugliedern und nur noch im Spezialhaus zu führen

- Abteilungen bzw. Warengruppen sehr stark in der Filiale zu reduzieren und den entstehenden Kaufwunsch im Spezialhaus zu befriedigen

- Spezialhäuser unter Beibehaltung des eigenen Namens (z.B. Karstadt-Möbelhaus) oder

- Spezialhäuser unter fremdem Namen zu eröffnen ${ }^{3}$.

Die Realisierung dieser Strategien ist sehr stark von standortbezogenen situationalen Faktoren abhängig. So werden in der realen Warenhausplanung zwei dominante Beweggründe für die Realisierung von Branching-out-Strategien unterschieden. Der erste Grund betrifft Abteilungen, die durch

1 Dies wird häufig als wesentlicher Grund für die aktuell schwache Ertragssituation der Warenhäuser angesehen. Vgl. z.B. Höller, P., a.a.O., S. 14; Krüger, H., a.a.O., S. 64 .

2 Als "Vorreiter" dieser Strategie kann Karstadt gelten. Dieser Konzern betrieb 197915 Spezialhäuser: 1 Möbelhaus, 5 Einrichtungshäuser (Möbel und Heimtextilien), 2 Technikhäuser, 6 Sport- und Freizeithäuser sowie 1 Boutique. Vgl. o.V., Sortimentsfight statt Flächenklotzerei, a.a.o., s. 7 .

3 Damit vollziehen sie eigentlich den Ubbergang zu einer anderen Betriebsform (Beispiel: Mauritius-Moden der Kaufhof $A G)$. 
besonders gute Umsatzchancen gekennzeichnet sind, so daß der Markt am besten mit einem Spezialhaus ausgeschöpft werden kann. Der zweite Anlaß liegt in einer sehr stark angespannten örtlichen Wettbewerbssituation verbunden mit einer relativ schwachen eigenen Marktstellung begründet, so daß die Fortführung der Warengruppe bzw. Abteilung in der Filiale nicht mehr sinnvoll erscheint.

Eine endgültige Entscheidung über eine Branching-outStrategie muß auf einer detaillierten Analyse der Vorund Nachteile ihrer Realisierung beruhen. Hier sind insbesondere die Auswirkungen auf die Marketingziele detailliert zu untersuchen. So kann z.B. die Umwandlung von Trendabteilungen in Spezialhäuser für die einzelne Filiale, in denen die Abteilung bisher geführt wurde, einen Attraktivitätsverlust bedeuten.

Zusätzlich ist $\mathrm{zu}$ analysieren, ob die betrachtete $\mathrm{Ab}-$ teilung sehr starke Verbundwirkungen $\mathrm{zu}$ anderen Abteilungen aufweist und von daher eine Ausgliederung den Erfolg anderer Abteilungen beeinträchtigt ${ }^{1}$. Ferner sind die möglichen Konkurrenzreaktionen in der Planung $\mathrm{zu}$ berücksichtigen. Bezieht man schließlich noch die prognostizierten Zielwirkungen im Hinblick auf Ertrag, Marktanteil, Kundenfrequenz usw. mit ein, so ergibt sich eine abgerundete Beurteilung einer warengruppen- bzw. abteilungsorientierten Spezialhausstrategie.

1 Dahinter steht u.a. die bislang noch nicht eindeutig belegte Hypothese, daß jedes Warenhaus sog. frequenzbringende Warengruppen besitzt. Diese sorgen dafür, daß der potentielle Kunde das Haus betritt und somit die Kaufvoraussetzung auch in anderen Abteilungen geschaffen wird. 
Im Rahmen der Standortneuwahl stellt sich ebenso die Frage nach der Größe der neu zu gründenden Filiale. Differenziert man die Filialgrößen nach der jeweiligen Verkaufsfläche grob in fünf Typen 1 , so muß sich die Warenhausleitung für einen bestimmten Filialtyp entscheiden. Dabei erfolgt die Entscheidung primär unter dem Gesichtspunkt der wahrscheinlichen zukünftigen Zielerfüllung. Greift man beispielhaft die Zielsetzung Umsatz pro gm einer neuen Filiale heraus, so zeigt eine Analyse der Äusprägungen dieses $\mathrm{Zieles}$ in verschiedenen Filialgrößenklassen cieutliche Unterschiede (vgl. Tab. 11 ).

\begin{tabular}{|l|c|}
\hline $\begin{array}{l}\text { Filialgrößen } \\
\text { (in qm) }\end{array}$ & $\begin{array}{l}\text { Umsatz pro qm } \\
\text { (in DM) }\end{array}$ \\
\hline 1.0 - 4000 & 420 \\
2.4000-6000 & 477 \\
3. $6500-9000$ & 486 \\
4. $9000-13000$ & 551 \\
5.13000 und mehr & 581 \\
\hline
\end{tabular}

${ }^{+}$Durchschnitt über alle 4 Warenhauskonzerne (je Monat) Tabelle 11 : Umsatz pro qm nach Filialgrößenklasser ${ }^{2}$

1 Siehe hierzu die Größenklassen (Typen) in der Tabelle 11

2 Quelle: Angaben der Marktforschungsabteilung eines Warenhauskonzerns sowie eigene Berechnuriren. Die Angaben in der Tabelle beziehen sich auf das Jahr 1969. Zeitreihenanalysen bestätigen die Gültigkeit der Aussage (auf höherem Niveau der Wertangaben) auch für die nachfolgenden Jahre. 
Demnach sind auf den ersten Blick Filialen mit relativ großer Verkaufsfläche besonders realisierungswürdig. Aufgrund der zahlreichen externen Beschränkungen haben die Warenhäuser in den vergangenen Jahren jedoch untere Filialgrößenklassen stark ausgebaut, um die Expansionsstrategie zu realisieren ${ }^{1}$. Zieht man zur Beurteilung dieser Strategie zusätzlich die Nebenbedingung der zielgruppenprofilierung hinzu, so wird die Gefahr unmittelbar deutlich: Die relativ geringe Verkaufsfläche ermöglicht lediglich die Realisierung einer atypischen Sortimentsstruktur, die hinsichtlich der Sortimentsbreite und -tiefe eine deutlich geringere Ausprägung aufweist als das "typische" Warenhaus. Die Erwartungshaltung der Käufer, die vom normalen, typischen (d.h. hier großflächigen) Warenhaus geprägt ist, kann hier nicht erfüllt werden. Unter dieser Perspektive ist ein Verzicht auf eine starke Expansionsorientierung für Warenhäuser zu überprüfen. Auch die reale Entwicklung der vergangenen zwei Jahre scheint dafür $z u$ sprechen, daß diese Strategie nicht mehr in dem Maße wie bisher verfolgt wird ${ }^{2}$.

Die expansionsorientierte Standortanpassung verlangt die Erweiterung einer Filiale an einem vorhandenen Standort. Restriktive städtebauliche Verordnungen auf der einen Seite sowie das in der Regel vergebene Geschäftsumfeld setzen meist einer spürbaren Verkaufsflächenausweitung auch hier deutliche Grenzen ${ }^{3}$.

1 Vgl. Krüger, H., a.a.0., S: 64 .

2 Als Indikator dient die $\mathrm{Zahl}$ der Neueröffnungen von Filialen, die in den vergangenen Jahren deutlich niedriger lag als jemals zuvor (vgl. die Angaben in den Geschäftsberichten der Warenhauskonzerne).

3 In der Regel sind nur geringe Korrekturen z.E. durch die Umwandlung von Lager- in Verkaufsräume möglich. 
Vor diesem Hintergrund rücken Konsolidierungsstrategien als Basisstrategien stärker in den Vordergrund. Diese beinhalten eine eindeutige Ausrichtung der standortbezogenen Strategien auf erfolgsbestimmende Kennzahlen (z.B. Filialrohertrag, DB, Umsatz pro qm usw.). Dementsprechend orientiert sich auch die Beurteilung neuer standorte stärker an ertragsorientierten Zielsetzungen. Daraus resultiert zwar eine Verringerung der insgesamt zur Verfügung stehenden Standorte, die dann aber durch das Primat erfolgsorientierter Kriterien bei ihrer Besetzung einen höheren Erfolgsbeitrag versprechen.

Diese Verhaltensweise findet gerade in der letzten Zeit in Filialumschichtungsstrategien der Warenhäuser ihren Ausdruck. Diese beinhalten im Hinblick auf ein ausgewogenes Filialsystem - ähnlich wie die Sortimentsumschichtungen - die Stärkung erfolgreicher Filialen bzw. Filialgruppen, die Eliminierung schwacher Filialen sowie die Eröffnung neuer Filialen. Die Intensität der Filialumschichtung ist neben den ertragsorientierten zielsetzungen primär von den oben diskutierten Restriktionen abhängig. Darüber hinaus hängt sie sehr stark von den subjektiven Vorstellungen des zielsetzenden Gremiums ab und entzieht sich somit einer generellen Empfehlung. Allerdings müssen bei dieser Strategie negative Auswirkungen auf die Marktstellung (gemessen am Marktanteil oder dem regionalen Präsenzgrad) in Kauf genommen werden, zumal die Warenhauskonkurrenten in der Regel eine andere Strategie verfolgen werden.

Strategische tberlegungen der Warenhäuser in Bezug auf Erhaltungs-/Schrumpfungsstrategien sind in der realen Situation bislang selten $z u$ beobachten. Idealtypischerweise beziehen sich diese auf eine Erhaltung der Anzahl der Verkaufsstätten bzw. auf ihre Verringerung. Für einen einzelnen Warenhauskonzern ist damit solange ein 
Marktanteilsverlust verbunden, wie die übrigen Warenhäuser oder Betriebsformen eine andere Strategie realisieren. Bei einer Eliminierungsstrategie nimmt die Verkaufsfläche sogar absolut ab. Bei der aktuell großen Bedeutung der Ziele des Umsatz- und Verkaufsflächenwachstums in Warenhäusern sind diese strategien nicht realisierungswürdig, zumal sie auch im Hinblick auf die Zielgruppenbearbeitung (wenn $z$.B. bestimmte standorte nicht besetzt sind) sowie auf die Profilierung gegenüber der Konkurrenz (z.B. durch Marktanteilsverluste) negative Ausstrahlungseffekte verursachen.

Unabhängig von der endgültigen Standortstrategie gilt der Pflege und Stärkung der Marktstellung an bereits besetzten Standorten das besondere Interesse der Warenhausleitung. Diese ist Gegenstand der verkaufsstättenbezogenen Marketingstrategie.

\subsection{Verkaufsstättenbezogene strategien}

Im Mittelpunkt stehen hier die La dengestaltung im $\mathrm{zu}$ sammenhang mit notwendigen Umbaumaßnahmen sowie die innerbetriebliche Standortwahl (Flächenschlüssel)1. Diese Instrumente sind unabhängig von der gewählten Art der Basisstrategie in jedem Falle zur Absicherung der gegenwärtigen Marktstellung einzusetzen.

Aufgabe der Geschäftsstättengestaltung ${ }^{2}$ ist es, die Filiale durch ein handelsspezifisches Image in den Augen der

1 Unter dem Flächenschlüssel versteht man in der Warenhausplanung die prozentuale Aufteilung der gesamten Verkaufsfläche auf einzelne Artikel- bzw. Warengruppen.

2 Vgl. zu diesem Problemkreis z.B. Bearchell, C.A., a.a.O., S. 84 ff.; Davidson, W.R., et al., a.a.O., S. $516 \mathrm{ff.;}$ Meffert, H., Bedeutung, Aufgaben und Zukunftsperspektiven des Ladenbaus im Rahmen des Handelsmarketing, unveröffentlichtes Manuskript, Münster 1979 . 
Kunden eindeutig $z u$ profilieren und damit unverwechselbar von den Konkurrenzfilialen abzuheben. Darüber hinaus hat sie für das gesamte Filialsystem der Warenhäuser die Funktion, durch einen einheitlichen Stil insbesondere in der Fassadengestaltung zur Identifizierung an unterschiedlichen Standorten beizutragen. Die innerbetriebliche Gestaltung ist. ein wichtiges Instrument zur Herbeiführung einer angenehmen Einkaufsatmosphäre, auf die der Warenhauskunde besonderen Wert legt. Unter dieser Perspektive muß die Ladengestaltung einen kaufstimulierenden Rahmen für das Sortiment bieten, zu einer für den Kunden übersichtlichen Warenpräsentation führen, die gewünschte Betonuny aktueller Artikel- oder Artikelgruppen jederzeit ermöglichen und damit gleichzeitig die Abwicklung verkaufsfördernder Maßnahmen erlauben. Weiterhin muß die Ladengestaltung eine ausreichende Flexibilität gewähren, da die aktuelle Geschäftseinrichtung eine wesentliche Imagekomponente eines erfolgreichen Handelsbetriebes darstellt ${ }^{1}$.

Während die Geschäftsstättengestaltung in erster Linie die Erreichung des Imagezieles eines Warenhauses unterstützt, dient die innerbetriebliche Standortwahl bzw. die Vorgabe eines detaillierten Flächenschlüssels ertragsorientierten zielsetzungen. In diesem zusammenhang ist für jede Einzelfiliale zu überprüfen, inwieweit Ertragsverbesse-

1 So zeigt eine empirische Untersuchung, daß Ladeneinrichtungen erfolgreicher Betriebe nur eine halb so hohe durchschnittliche Lebenserwartung haben wie die von leistungsschwachen Betrieben. Vgl. hierzu Berger, S., a.a.O., S. $36 \mathrm{ff..} \mathrm{Zu} \mathrm{ähnlichen} \mathrm{Ergebnissen} \mathrm{gelangt}$ auch Barth, K., Unternehmensführung, a.a.O., S. 177. 
rungen durch eine andere Verkaufsflächenaufteilung möglich sind. Zielsetzung ist dabei eine Ertragsoptimierung pro qm Verkaufsfläche. Der Flächenschlüssel gibt in Form eines abteilungsweisen Soll-Ist-Vergleichs an, wie die Flächenverteilung in jeder Filiale - basierend auf den Durchschnittswerten der vergangenen Jahre - aussehen müßte. Aus den Differenzen zwischen Soll und Ist ist zu entnehmen, durch welche Flächenveränderung der Filialertrag gesteigert werden kann.

Für die Flächenvorgabe als einem in den Filialen sehr wichtigen Steuerungsinstrument zur Realisierung ertragsorientierter zielvorstellungen gilt andererseits, daß sie selbstverständlich unter Berücksichtigung aller hausspezifischen und standortrelevanten Besonderheiten erfolgen muß. In der realen Planungssituation sollte die Zweckmäßigkeit maßgeblicher Flächenveränderungen in jedem Fall durch Wirtschaftlichkeitsrechnungen nachgewiesen werden, ebenso wie Flächenreduzierungen nur bei ersatzweisem Ausbau ertragsstarker Abteilungen zulässig sind ${ }^{1}$.

1 Dieser Problemkreis hängt natürlich eng mit der für das gesamte Unternehmen geltenden Sortimentsstrategie zusammen und stellt in der Warenhauspraxis ein oftmals schwieriges Abstimmungsproblem zwischen der Zentrale und den Filialen dar. Da sich die Sortimentsstruktur einer Filiale an den lokalen Daten (Filialgröße, Standort, bauliche Eigenschaften usw.) orientieren muB, wird sich in der Realität eine mehr oder weniger starke Änderung zur zentralseitig erwünschten Sortimentsstruktur ergeben. Die realisierte Abweichung ist vom Entscheidungsspielraum des Filial- bzw. SGEManagement bzw. vom realisierten Planungssystem abhängig und stellt damit im weitesten Sinne ein organisatorisches Problem dar (siehe hierzu auch Kapitel 6 dieser Arbeit). 
Nach vorläufiger Festlegung der distributionspolitischen Marketingstrategien müssen diese mit den sortimentspolitischen Entscheidungen abgestimmt werden. Durch einen mehrfachen rückgekoppelten Generierungs- und Selektionsproze $B$ muB ein konsistentes system von sortimentsund distributionspolitischen strategien entwickelt werden, das die Konkretisierung der kontrahierungsund kommunikationspolitischen Marketingstrategien determiniert. Darüber hinaus orientiert sich diese zweite Planungsstufe von Marketing-Instrumentalstrategien in besonderem Maße an der vorgelagerten Positionierungsentscheidung ${ }^{1}$.

\subsection{Entwicklung kontrahierungspolitischer Strategien von Warenhäusern}

Im Rahmen der kontrahierungspolitischen Marketinginstrumente kommt unter strategischem Blickwinkel der Preispolitik $^{2}$ im Handel die größte Bedeutung zu. Ihr gegenüber erweisen die übrigen Instrumente Rabatt ${ }^{3}$, Kredit ${ }^{4}$, Lieferungs- und Zahlungsbedingungen ${ }^{5}$ sowie Ga-

1 Vgl. hierzu Kapitel 5.1 der Arbeit.

2 Vgl. zum Utberblick z.B. Bearchell, C.A., a.a.O., S. 123 ff.; Bolen, W.H., a.a.O., S. 203 ff.; Buskirk, R.H., Buskirk, B.D., a.a.O., S. $251 \mathrm{ff.;}$ Davidson, W.R. et al., a.a.O., S. $240 \mathrm{ff.;}$ Falk, B.R., Wolf, J., Handelsbetriebslehre, a.a.O., S. $267 \mathrm{ff}$; Hansen, U., Marketing 2, a.a.0., S. 327 ff.; Mangold, H.G., a.a.O., S. $123 \mathrm{ff..}$

3 Vgl. z.B. den Überblick bei Ahlert, D., Marketing, a.a.o., S. $129 \mathrm{ff.;}$ Hansen, U., Marketing 2, a.a.O., S. $379 \mathrm{ff.;}$ Meffert, H., Marketing, a.a.O., S. $316 \mathrm{ff..}$

4 Vgl. z.B. den Überblick bei Ahlert, D., Marketing, a.a.O., S. $145 \mathrm{ff}$; d derselbe, Absatzförderung durch Absatzkreditpolitik, wiesbaden 1972 (im folgenden zitiert als: Ahlert, D., Absatzpolitik); Buskirk, R.H., Buskirk, B.D., a.a.O., S. 423 ff.; Hansen, U., Marketing 2, a.a.0., S. $407 \mathrm{ff} .$.

5 Vgl. z.B. den Überblick bei Ahlert, D., Marketing, a.a.O., S. 156 ff.; Mangold, H.G., a.a.O., S. 205 ff.. 
rantie stark operativen Charakter auf (kurzfristig variierbar, stark von der örtlichen situation abhängig usw.), sind teilweise relativ unbedeutend für Warenhäuser (z.B. Rabatte) und unterliegen teilweise nichtkontrollierbaren Einflüssen (z.B. gesetzliche Regelungen der Lieferungs- und Zahlungsbedingungen sowie der Garantie). Insofern stellt die Preispolitik das einzige strategisch $\mathrm{zu}$ planende kontrahierungspolitische Instrument dar.

Entsprechend der zugrundeliegenden Planungssystematik mu $\beta$ sich die Preispolitik unmittelbar an den sortimentspolitischen Strategien ausrichten wie die Abbildung 26 zeigt. Liegen als sortimentspolitische Strategien eine mittlere bis hohe Sualitätslage, ein relativ hoher Markenanteil sowie ein breites und tiefes Sortiment vor,

Sortimentsniveau

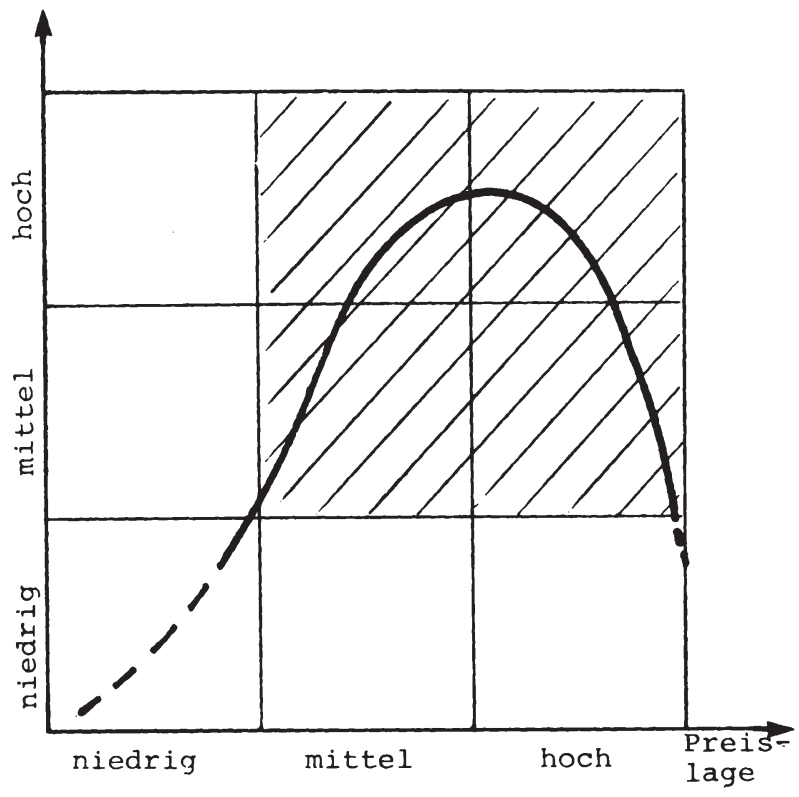

Abb. 26: Zusammenhang zwischen Sortimentsniveau und Preislagenstrategie 
was ein hohes Sortimentsniveau impliziert, so sind als adäquate preispolitische Strategie die meisten Artikel des Sortiments in der gehobenen Preislage ${ }^{1} \mathrm{zu}$ plazieren. Die Realisierung dieser Preislagenstrategie bringt Vorteile im Hinblick auf die Profilierung gegenüber den Konkurrenten und zwar insbesondere gegenüber jenen Betriebsformen, die eine niedrige Preislage bevorzugen (SB-Warenhäuser, Discounter) ${ }^{2}$. Sie entspricht darüber hinaus auch weitgehend den Anforderungen der Basiszielgruppe an Preisund Qualitätslagen im Warenhaus. Hierdurch bietet sie zusätzlich die Chance einer Erschließung der Zielgruppe in Richtung einer höheren sozialen Schicht.

Im Rahmen der unmittelbaren Konkurrenzstrategie muß jeder Warenhauskonzern entsprechend seiner Ziele versuchen, die höchsten, nachhaltig am Markt realisierbaren Preise durchzusetzen. Die Preisfestsetzung orientiert sich nicht an der Verhaltensweise der Konku-renz, sondern primär an den eigenen zielsetzungen und den Preisvorstellungen der Kunden. Diese Vorgehensweise wird als Strategie der Preisführerschaft bezeichnet und kann realistischerweise nur für einen Warenhauskonzern nur in Teilbereichen Leitmaxime sein.

1 Hierunter ist die Reduzierung von kontinuierlich gestreuten Preisreihen auf wenige Preisschwerpunkte, zwischen denen merkliche Preissprünge entstehen, zu verstehen. Vgl. Gümbel, R., Sortimentspolitik, a.a.0., S. $184 \mathrm{ff}$; Hansen, U., Marketing 2, a.a.O., S. 365; vgl. auch die warenhausspezifische Diskussion bei Mangold, H.G., a.a.O., S. $145 \mathrm{ff}$..

2 In der Beurteilung der Strategie, die untere Preislage auszubauen und den sich dort befindlichen Betriebsformen Gleiches entgegenzusetzen, ist man sich heute weitgehend einig: sie war "falsch" und hat wesentlich zur schlechten Ertragslage der Warenhäuser beigetragen. Vgl. hierzu z.B. Krüger, H., a.a.O., S. 64; Steffenhagen, H., Marktführer, a.a.O., S. 30. 
Wie die preisfolgerstrategie der übrigen Warenhauskonkurrenten sowie der anderen Betriebsformen auszusehen hat, ist situationsspezifisch $z u$ entscheiden und basiert auf einer detaillierten Analyse der jeweiligen Marktstellung sowie der spezifischen Stärken und Schwächen. Generell ist die Preisführer-Strategie warengruppenspezifisch differenziert $z u$ sehen: liegen spezifische Kompetenzen bzw. klare Profilierungen bei bestimmten Warengruppen vor, so erlaubt dies dort die Preisführerstrategie, während in weniger profilierten Warengruppen eine Preisfolgerstrategie eingeschlagen werden muß.

Der enge Zusammenhang zur Sortimentspolitik wird auch durch die Berücksichtigung des Warencharakters deutlich. Für Güter des täglichen Bedarfs' ist aufgrund ihrer relativ hohen Markttransparenz eher eine Preisfolgerstrategie zu empfehlen. Bei Shopping Goods ${ }^{2}$, bei denen die Käufer i.d.R. weniger preisbewußt kaufen, hängt die Kalkulation weniger von der Marktstellung im Vergleich zur Konkurrenz ab, sondern vielmehr vom "Wert" der Ware, den der Kunde ihr zumiBt.

Die Restriktion der distributionspolitischen Strategien findet dann ihren Niederschlag, wenn standortspezifische Faktoren $z u$ einer Modifizierung der zunächst festgelegten Preisstrategie zwingen. Als wesentliche Faktoren sind hier insbesondere die örtliche Käuferschaft, das allgemeine Preisniveau sowie die örtliche Konkurrenz zu nennen.

Die Gefahr der gehobenen Preislagenstrategie liegt in der schlechteren Beurteilung bei ler Imagedimension

1 Auch als Convenience Goods bezeichnet. Vgl. den grundlegenden Aufsatz im Hinblick auf handelspolitische Strategien von Bucklin, L., Retail Strategy and the Classification of Consumer Goods, in: JOM, Vol. 27, Jan. 1963, S. 5o-55.

2 Vgl. ebenda, S. 50. 
"Preiswürdigkeit". Dieser Problematik kann durch eine aktive Sonderpreisstrategie ${ }^{1}$ begegnet werden, die das zeitlich begrenzte Angebot spezieller Artikel zu besonders knapp kalkulierten Preisen vorsieht ${ }^{2}$. Durch ihren gezielten Einsatz ist die Leistungsfähigkeit im Sinne der Preiswürdigkeit unter Beweis zu stellen ${ }^{3}$ und somit diese Imagedimension in der gewünschten Weise zu stabilisieren. Da allerdings $z u$ häufige Sonderangebote zu einem Verlust des Qualitätsimage führen, kann die Sonderpreisstrategie nur punktuell eingesetzt werden ${ }^{4}$.

Das breite Warenhaussortiment wirft zuletzt die Frage nach der Strategie der Preisspreizung auf. Sie bringt den Abstand zwischen dem niedrigsten und dem höchsten Preis einer Artikelgruppe zum Ausdruck (z.B. Herrenhemden zwischen DM 18,- und DM 48,-). Im Hinblick auf die breite

1 Vgl. zu diesem Problemkreis z. B. Hansen, U., Marketing, a.a.O., S. 361 ff.; Bearchell, C.A., a.a.O., S. $134 \mathrm{ff.;}$ Bolen, W.H., a.a.O., S. $217 \mathrm{ff}$; Welzel, K., a.a.O., S. $37 \mathrm{ff}$.. Vgl. auch den engen Zusammenhang zwischen der Planung von Sonderangeboten bei Hersteller- und Handelsunternehmen z. B. bei Glinz, M., Sonderpreisaktionen des Herstellers und des Handels, Wiesbaden 1978.

2 Damit wird hier primär auf das Marketinginstrument "Preis" abgestelit. Im Grunde müssen jedoch auch die übrigen Marketinginstrumente -mit berücksichtigt werden. So muß z. B. der Sonderpreis kommunikativ herausgestellt, der Artikel adäquat präsentiert und die Präsenz am Standort sichergestellt werden usw.. Somit stellt sich die Sonderpreisstrategie in der realen Planung als eine Instrumentekombination dar. Vgl. hierzu auch die Diskussion bei Welzel, K., a.a.O., S. $37 \mathrm{ff.}$.

3 Vgl. Petermann, G., Marketinginstrumentarium, a.a.o., S. $163 \mathrm{f}$..

Betrachtet man die Realisierung dieser Strategie in den Warenhäusern in den vergangenen Jahren, so kann man sich des Eindrucks eines "überzogenen" Einsatzes der Sonderangebotsstrategie nicht erwehren. 
Zielgruppe der Warenhäuser ist eine größere Preisspreizung als in anderen Betriebsformen zu realisieren. Auch hier ergeben sich warengruppenspezifische Ansatzpunkte: strebt die Warenhausleitung ein gehobenes Preisniveau an, so wird sie eine größere Preisspreizung beschließen, da diese eher eine Ausweitung der Preislagen nach oben zuläßt.

Weisen die preispolitischen Strategien in ihrer Gesamtheit einen besonders engen zusammenhang zur sortimentspolitik auf, so ist die im folgenden $z u$ analysierende Kommunikationspolitik insbesondere im Hinblick auf distributionspolitische Strategien zu diskutieren.

\subsection{Entwicklung kommunikationspolitischer Strategien von Warenhäusern}

Ziel kommunikationspolitischer Aktivitäten ${ }^{1}$ ist es, sich positiv von den Konkurrenten abzuheben sowie den aktuellen und potentiellen Kunden seine Leistungsfähigkeit $z u$ demonstrieren. Damit kommt der Kommunikationspolitik eine wesentliche profilierende und imagebildende Aufgabe $z u$. Von den zur Verfügung stehenden kommunikationspolitischen Instrumenten weisen die klassische Werbung ${ }^{2}$,

1 Vgl. den Überblick bei Handelsunternehmen von Scheele, W., Kommunikationspolitik, in: Nieschlag, R., Eckardstein, D., Der Filialbetrieb als System. Das Cornelius-Stüssgen-Modell, Köln 1972, S. 231-262 sowie Mangold, H.G., a.a.O., S. 215 ff..

2 Vgl. hierzu z.B. Berman, B., Evans, J.R., a.a.O., S. 430 ff.; Bolen, W.H., a.a.O., S. 332 ff.; Davidson, W.R., et al., a.a.O., S. 402 ff.; Hansen, U., Marketing 2, a.a.o., s. 437 ff.. 
der persönliche Verkauf ${ }^{1}$, die Warenpräsentation ${ }^{2}$ sowie die Bedienungsform ${ }^{3}$ eindeutig strategischen Charakter auf. Einerseits sind sie langfristig wirksam und andererseits müssen sie sich wegen der zur Kundenbefriedigung notwendigen Kontinuität des Erscheinungsbildes bzw. Auftretens über einen längeren Zeitraum hinweg Gültigkeit besitzen ${ }^{4}$.

Im Mittelpunkt der kommunikationspolitischen Aktivitäten steht die klassische Werbung. Ihr kommt neben kurzEristig wirksamen Aktionsanstößen primär die Aufgabe $z u$, langfristig ein positives Vorstellungsbild des Warenhauses bei den aktuellen und potentiellen Kunden zu verankern. Dabei muß die Warenhausleitung eine zweckadäquate Kombination zwischen einer rein firmen- bzw. institutionsbezogenen Werbung und einer leistungs-

1 Vgl. z. B. Berman, B., Evans, J.R., a.a.O., S. $441 \mathrm{ff}$; Davidson, W.R. et al., a.a.O., S. $440 \mathrm{ff.;}$ Bolen, W.H., S. $269 \mathrm{ff}$..

2 Vgl. z. B.: Barth, K., Die Warenpräsentation in Einzelhandelsunternehmen, in: Mitteilungen des Instituts für Handelsforschung an der Universität zu Köln, hrsg. von E.Sundhoff, 27.Jg., 1975, Nr. 7, S. 93-97 (im folgenden zitiert als Barth, K., Warenpräsentation), Derselbe, Sortimente, a.a.O., S. $42 \mathrm{ff.;}$ Hansen, U., Marketing 1, a.a.O., S. 280 ff..

$3 \mathrm{Vgl.z}$. B. den guten Überblick bei Hansen, U., Marketing 1, a.a.O., S. $282 \mathrm{ff}$. sowie die dort angegebene Literatur.

4 Die Verkaufsförderung wird primär unter kurzfristig erfolgsorientierten Zielsetzungen eingesetzt und deshalb hier nicht weiter erörtert. Auch die Public Relation wird aufgrund des lediglich unterstützenden Charakters und der primären Ansprache von sog. sekundären Zielgruppen (Aktionäre, Gläubiger, sonstige öffentlichkeit) von einer detaillierten Betrachtung ausgeklammert. Vgl. zu diesem Instrument z. B.: Bolen, W.H., a.a.O., S. $351 \mathrm{ff.:}$ Berman, B., Evans, J.R., a.a.O., S. $444 \mathrm{ff} .$, Davidson, W.R., et al., a.a.O., S. $437 \mathrm{f}$..

$\mathrm{Zu}$ einer Interpretation der Public Relations als strategischem Instrument für Hersteller vgl. z.B. Hatter, S.St., Strategic Planning for Public Relations, in: LRP, Vol. 13, June 1980, S. 57-60. 
bzw. artikelbezogenen Werbung finden ${ }^{1}$. Während im ersten Fall das Warenhaus im Mittelpunkt der kommunikativen Aussage steht, gilt im zweiten Fall dem Artikel bzw. der Leistung das unmittelbare Interesse.

Im Hinblick auf das Profilierungsziel muß die Werbung zunächst das Warenhaus als Ganzes in den Augen der Konsumenten positiv erscheinen lassen. Da die aktuellen und potentiellen Käufer weniger an dem Warenhaus selbst, als vielmehr an der Leistung interessiert sind, muß ebenso die Leistungswerbung eingesetzt werden.

Die Entscheidung über die Gewichtung dieser beiden Elemente ist situationsspezifisch zu treffen und entzieht sich einer generellen Empfehlung. Tendenziell ist jedoch für die Praxis der Warenhauswerbung eine leichte Dominanz der Artikel- bzw. Leistungswerbung festzustellen. Bei einer räumlichen Differenzierung der Werbestrategie greift allerdings auf der nationalen Ebene eher die Firmenwerbung Platz, während am lokalen Standort die Artikel-bzw. Leistungswerbung dominiert.

Umso wichtiger erscheint deshalb die Entwicklung eines einheitlichen Werbestils als kommunikative Basis. Er muß sich auf die Werbegestaltung, die Botschaft, die Headline, die Farbkomposition usw. beziehen und für alle Unternehmens- bzw. Planungsebenen gelten.

Im Rahmen der Werbegestaltung ist insbesondere auf die differenzierte Zielgruppenansprache $\mathrm{zu}$ achten. Diese ist durch eine divergierende Ansprache über unterschiedliche Sortimente mit differierenden Werbeargumenten zu erreichen (z.B. lebendiges Angebot und betriebsame Kaufatmosphäre für den Jugendmarkt). Hierdurch gelingt es zwar, die jeweils anvisierte Zielgruppe adäquat anzusprechen,

1 Vgl. zur Darstellung dieses Entscheidungsproblems: Hansen, U., Marketing 2, a.a.O., S. $441 \mathrm{ff}$. sowie die dort angegebene Literatur. 
doch liegt das besondere Problem für die Warenhäuser darin, zu verhindern, daß sich bei den übrigen - ebenfalls mit dem Werbeträger in Kontakt kommenden - Zielgruppen negative Wirkungen ergeben ${ }^{1}$.

Die Zielsetzung, die aktuelle Zielgruppe um Randsegmente zu erweitern ${ }^{2}$, muß ihren kommunikativen Niederschlag in einer expansiv orientierten Werbestrategie finden. So impliziert eine Sortimentsanhebung konsequenterweise eine Erweiterung der Zielgruppe um höher angesiedelte käuferschichten, die nur durch zusätzliche, speziell auf sie zugeschnittene Werbemaßnahmen erreicht werden kann.

Neben der unpersönlichen Kommunikation rückt in jüngster Zeit der persönliche Verkauf ${ }^{3}$ in den Vordergrund, da qualifiziertes Verkaufspersonal in der Lage ist, individuell modifizierte, d.h. auf den einzelnen Kunden zugeschnittene Informationen $z u$ übermitteln und damit den Kaufentscheidungsproze $\beta$ wesentlich $z u$ beeinflussen. Insbesondere unter dem Aspekt der Profilierungsstrategie gegenüber den Konkurrenten stellt sich für Warenhäuser die Frage nach dem $z$ u realisierenden Umfang bzw. dem Niveau des persönlichen Verkaufs.

1 Angesichts der Bedeutung dieses Problems verwundert es nicht, da $\beta$ Warenhäuser in der realen situation einen Betriebsformenwechsel vollziehen (z.B. Kaufhof mit Mauritius-Moden) um so eine klare Trennung zwischen Warenhauszielgruppe und neuer Zielgruppe im kommunikativen Konzept zu ermöglichen.

2 Vgl. hierzu die Diskussion im Kapitel 3.1 der Arbeit.

3 Vgl. hierzu z.B. Freese, G., Verkäufer, a.a.0., S. 18; Hebbering, B., a.a.O., S. 23 ff.. Herder, H.von, Bedienung - der wiederentdeckte Bedarf?, in: Rationeller Handel 1/1980, S. 13-17. 
Da Imageuntersuchungen ${ }^{1}$ eine deutliche Schwäche der Warenhäuser im Hinblick auf den Umfang und die Qualität des Verkaufspersonals aufzeigen, ist durch Schulungsmaßnahmen und eine Erhöhung des Personalbestandes eine deutliche Verbesserung anzustreben. In der realen Situation ist im Hinblick auf die ertragsorientierten Zielsetzungen der Warenhäuser wegen der hohen Personalkosten nur eine Strategie der qualitativen Verbesserung des persönlichen Verkaufs $z$ u realisieren.

Die notwendige Begrenzung des Verkaufspersonals erfordert einennach Warengruppen differenzierten Einsatz von Fremäbeciienung, Vorwahl mit Beratungsmöglichkeit und Selbstbedienung. Dort, wo sich die Ware selbst mittels Verpackung, Text oder Display-Material verkaufen läßt, ist die Selbstbedienung oder die Vorwahl mit Beratungsmöglichkeit $z$ u realisieren. Lediglich bei besonders problemvoller, hochwertiger ware ist auf die reine Fremdbedienung zurückzugreifen.

In engem Zusammenhang mit der Bedienungsform ist die Warenpräsentation $^{2} \mathrm{zu}$ sehen. Insbesondere bei Selbstbedienung muß die Ware im Verkaufsraum bzw. im Regal so präsentiert werden, daß der Käufer sie selbständig finden, prüfen und eine Entscheidung über Kauf oder Nichtkauf zu treffen vermag.

1 Schröder beschreibt diese strategie folgendermaßen: "... die Vorinformation der Kunden auch bei gehobenem Niveau so verbessern, .. daß das eigentliche Verkaufsgespräch oder die Beratung erst im letzten Drittel der Entschlußzeit des Kunden geführt wird." Schröder, B., Das Warenhaus der Zukunft (II), in: BddW Nr. 128, 21.6.1978, S. 1.

2 Vgl. hierzu die grundlegenden Ausführungen von Barth, K., Warenpräsentation, a.a.0., S. $93 \mathrm{ff}$. 
5.6 Problem der Auswahl und Kombination der Marketing-Instrumentalstrategien in Warenhäusern

Mit den skizzierten Marketing-Instrumentalstrategien steht der Warenhausleitung ein breites Spektrum von Strategiealternativen zur Verfügung, dessen logisch-kreative Ableitung wesentlich von den zuvor festgelegten Basisstrategien und damit von der unternehmensspezifischen situation bestimmt wird. Die entwickelten Marketing-Instrumentalstrategien sind nun im Hinblick auf ihre Ubereinstimmung mit den unternehmensspezifischen Faktoren $z u$ überprüfen. Diese Bewertung und Auswahl der Strategiealternativen muB insbesondere im Hinblick auf die Unternehmensziele vorgenommen werden. Nur jene Strategien sind zu realisieren, die einen Beitrag zur Zielerfüllung leisten. Liegen alternative Strategien vor, ist jener der Vorzug zu geben, die den größten Beitrag zur Zielerreichung bringt.

Die Bewertung und Auswahl der Marketingstrategien anhand ihrer Zielerreichungsgrade stellt die Warenhausleitung zunächst vor das Problem, die notwendigen Informationsgrundlagen zu schaffen. Bereits in der Planungsphase müBte die Wirkungsweise alternativer Marketingstrategien hinsichtlich bestimmter Ziele (z.B. Kundenfrequenz, Umsatz, Dekkungsbeitrag) - mithin die Marktaktionsfunktion ${ }^{1}$ - bekannt sein $^{2}$. Die Kenntnis der Marktreaktionsfunktion ist in der realen Planungspraxis jedoch als Prämisse kaum aufrechtzuerhalten. Insbesondere bei strategischen Fragestellungen

1 Die Ermittlung der Marktreaktionsfunktion stellt im Grunde das zentrale Anliegen der Narketingtheorie dar; vgl. hierzu Meffert, H., Marketing, a.a.O., S. 89 ff.. Zum aktuellen Stand der Forschung vgl. Steffenhagen, H., Wirkungen, a.a.O., s. 9 ff..

2 Selbstverständlich sind neben zielwirkungen für praktische Problemstellungen noch weitere Informationsbereiche wie z.B. Konkurrenzreaktionen, Mitarbeitermotivation usw. interessant. Für das Entscheidungskalkül erscheinen jedoch die Zielwirkungen von primärer Bedeutung. 
ist die Warenhausleitung darauf angewiesen, die Bewertung und Auswahl von Strategien auf der Basis grob geschätzter Zielwirkungen vorzunehmen. Dabei hat es sich bei realen Planungsproblemen als nützlich erwiesen, die Zielwirkungen unter offenlegung der Planungsprämissen (z.B. Inflationsrate, Einkommensprognosen, Bevölkerungsentwicklung, Kostenentwicklung) - alternative Szenarien ${ }^{1}$ zu zeichnen, die zu alternativen $\mathrm{Zielwirkungen} \mathrm{führen.} \mathrm{Ubber} \mathrm{eine} \mathrm{von} \mathrm{Experten}$ vorgenommene Beurteilung der Eintrittswahrscheinlichkeit der Szenarien im Sinne der tatsächlichen Planungssituation ist es dann möglich, eine möglichst realistische zielentwicklung $\mathrm{zu}$ beschreiben und die Auswahl der erfolgversprechenden Strategien vorzunehmen.

In diesem Zusammenhang spielen für Warenhäuser langfristige Zielstrukturanalysen bzw. Zielprojektionen eine zentrale Rolle. Sie zeigen die geschätzte Entwicklung einer oder mehrerer Zielgrößen in Abhängigkeit von einer spezifischen Marketing-Instrumentalstrategie oder einer Strategiekombination über mehrere Jahre hinweg für eine spezifische Marketingsituation (Szenario) auf. Die aufgezeigte Planungssystematik legt dabei eine differenzierte Analyse im Hinblick auf die abgegrenzten strategischen Geschäftseinheiten nahe. Die Warenhausleitung muß die projektierten globalen Marketingziele wie z.B. den Gesamtumsatz, den Gesamtdeckungsbeitrag usw. auf die Warengruppen bzw. Filialgruppen beziehen, da die entwickelten Marketingstrategien diesem Aggregationsgrad entsprechen. Die Tabelle 13 verdeutlicht diese tberlegungen an einem Beispiel ${ }^{2}$.

1 Die Szenario-Technik wird inzwischen verstärkt in der strategischen Marketingplanung eingesetzt. Vgl. zu diesem Problemkreis z.B. Lehnen, F., Die Szenario-Technik in der Unternehmensplanung, in: $\mathrm{Z} f \mathrm{bF}$-Kontaktstudium 31 (1979), S. 71-75; Nair, K., Sarin, R.K., Generating Future Scenarios - Their Use in Strategic Planning, in: LPR, Vol. 12, June 1979, S. 57-61 sowie die jeweils angegebene Literatur.

2 Dieses Beispiel basiert nicht auf realen Warenhausdaten, sondern ist gedanklich entwickelt. Allerdings dürften in der Praxis durchaus ähnliche Strukturen aufzufinden sein. 
Die Leitung des Warenhauses $X$ muß davon ausgehen, daß aufgrund der geänderten Betriebsformenlandschaft, dem veränderten Verbraucherverhalten sowie der weitgehenden Besetzung lukrativer standorte eine expansionsorientierte Strategie wie in den vergangenen 10 Jahren (vgl. die Zahlenangaben des hypothetischen Beispiels in Tabelle 13) nicht mehr zu realisieren ist. Vielmehr muß der Schwerpunkt der Aktivitäten auf eine Konsolidierung ausgerichtet sein. Dabei erlangen insbesondere jene Úberlegungen Bedeutung, die auf eine Verbesserung der Ertragslage hinwirken.

Diese übergeordneten Strategien sind auf der Ebene der strategischen Geschäftseinheiten (hier Warengruppen) zu spezifizieren. Unter Berücksichtigung der aufgrund der Portfolio-Analyse festgelegten Marketing-Basisstrategien werden für die einzelnen Warengruppen die folgenden Wachstumsraten für die kommenden 5 Jahre festgelegt (vgl.Tab. 12).

\begin{tabular}{|c|c|c|c|c|}
\hline \multirow{2}{*}{$\begin{array}{l}\begin{array}{l}\text { Relevante } \\
\text { Struktur- } \\
\text { größen }\end{array} \\
\text { Warengruppe }\end{array}$} & \multicolumn{4}{|c|}{ jährliche Wachstumsraten } \\
\hline & VKF & $\mathrm{U}$ & $\mathrm{DB}$ & \\
\hline 1 & 48 & 10 & 12 & \\
\hline 2 & 58 & 9 & 14 & 8 \\
\hline 3 & 3,58 & 7 & 10 & 8 \\
\hline 4 & 28 & 6 & 8 & 8 \\
\hline 5 & 38 & 5 & 6 & 8 \\
\hline 6 & 48 & 8 & 6 & 8 \\
\hline 7 & 28 & 6 & 9 & 8 \\
\hline 8 & 68 & 12 & 15 & 8 \\
\hline 9 & 3 & 7 & 8 & 8 \\
\hline 10 & 18 & 5 & 7 & 8 \\
\hline
\end{tabular}

Tab 12: Wachstumsraten relevanter Größen als Prämissen für die Strukturanalyse

Mittels aieser festgelegten Wachstumsraten lassen sich die jeweiliegen Projektionen wie in der Tabelle 13 ermitteln.eln. Die für 1985 ermittelten werte zeigen dabei eindeutig die Modifikation von einer reinen Expansionsstrategie hin $\mathrm{zu}$ einer Strategie des deckungsbeitragsorientierten "gebremsten" Wachstums. Dies belegen insbesondere die Gesamtwerte, die für die Verkaufsflächen ein Wachstum von durchschnittlich 2,3 \& pro Jahr, für den Umsatz von 9,2 \& und für den Deckungsbeitrag von 12,5 \& anstreben. Dabei verschiebt sich die Verkaufsflächenstruktur deutlich zu den ertragsstarken Warengruppen, insbesondere die Warengruppen Damenkleidung (1), Herrenkleidung (2), und verschiedener Bedarf (8) werden ausgebaut. Die ertragsschwachen Warengruppen Schuhe und Kleintextilien (4), Einrichtungszubehör (7) und Restaurant/Café (10) verlieren dagegen weiterhin an Bedeutung. 


\begin{tabular}{|c|c|c|c|c|c|c|c|c|c|c|c|c|c|c|c|c|c|c|c|c|c|c|}
\hline \multirow{3}{*}{$\begin{array}{l}\text { Maren- } \\
\text { gr uppen }\end{array}$} & \multicolumn{6}{|c|}{ Verkaufs ilsche } & \multicolumn{9}{|c|}{ Unestr } & \multicolumn{6}{|c|}{ Deckungsbeitrag } & \\
\hline & \multicolumn{3}{|c|}{ abs. (In TSD gm) } & \multicolumn{3}{|c|}{ Ante11 (In I) } & \multicolumn{3}{|c|}{ abs. (In M10 DM) } & \multicolumn{3}{|c|}{ Antell (ln 1$)$} & \multicolumn{3}{|c|}{$\begin{array}{l}\text { Umalatz pro qm } \\
\text { (pro Jahr? in DA) }\end{array}$} & \multicolumn{3}{|c|}{ abs. (In M1O DM) } & \multicolumn{3}{|c|}{ Ante11 (In 1$)$} & \\
\hline & 1970 & 1980 & 1985 & 1970 & 1980 & 1985 & 1970 & 1980 & 1985 & 1970 & 1980 & 1985 & 1970 & 2980 & $19 A 5$ & 1970 & 1980 & 1995 & 1970 & 1980 & 1985 & \\
\hline 1. Damenkloldung & 30 & 56 & 68,1 & 14,1 & 13,6 & 14. & 230 & 430 & 693 & 28,0 & 14,6 & 16,0 & 2667 & 7678 & 10176 & 60 & 120 & 211 & 19,9 & 17,4 & 18,8 & $\cdots$ \\
\hline 2. Herrenk leldung & 16 & 30 & 30.5 & 7.5 & 7,3 & 7.5 & 120 & 230 & 354 & 9, & 7,8 & $\theta, 2$ & 7500 & 7667 & 9243 & 30 & 65 & 125 & 9,9 & 9,4 & 11,1 & $\cdots$ \\
\hline $\begin{array}{l}\text { 3. Alles fUrs } \\
\text { Kind }\end{array}$ & 12 & 24 & 20,2 & 5,6 & 5,8 & 5.5 & 70 & 140 & 196 & 5,5 & 4,8 & 4,5 & 5634 & 5834 & 6877 & 20 & 80 & 64 & 6,6 & 5,8 & 5,7 & $\ldots$ \\
\hline 4. Schune & & & & & & & & & & & & & & & & & & & & & & \\
\hline $\begin{array}{l}\text { und k1T. } \\
\text { 3. Text11. }\end{array}$ & - & - & & - & - & - & • & $\bullet$ & - & - & - & $\bullet$ & • & • & • & • & • & • & - & - & $\bullet$ & $\cdots$ \\
\hline Binrichtungen & $\bullet$ & - & - & • & - & - & - & •i & - & • & - & $\bullet$ & $\bullet$ & • & • & - & - & - & - & • & - & $\cdots$ \\
\hline $\begin{array}{l}\text { 6. Ubrige } \\
\text { Binrichtungen }\end{array}$ & $\bullet$ & 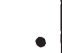 & 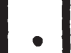 & & & & - & • & $\bullet$ & & & & & & - & $\bullet$ & • & - & - & & & $\cdots$ \\
\hline 7. Einrichtunga- & & & & & & & & & & & & & & & & & & & & & & \\
\hline zubehor & 32 & 58 & 64,0 & 15,2 & 14,1 & 13,2 & 180 & 420 & 562 & 14,1 & 14,3 & 13,0 & 5625 & 7241 & 8781 & so & 110 & $169^{\circ}$ & 16,5 & 16,0 & 15,0 & $\cdots$ \\
\hline B. Verschledener & & & & & & & & & & & & & & & & & & & & & & \\
\hline Bedarf & 23 & 58 & 77. & 10,8 & 14,1 & 26,5 & 120 & 400 & 705 & 9,4 & 13,6 & 16,3 & 5217 & 6897 & 9085 & 29 & 90 & 181 & 9.6 & 13,1 & 15,1 & $\cdots$ \\
\hline 9. Lebensmittel & 20 & 45 & 52.2 & 9,4 & 10,9 & 10,8 & 200 & 600 & 642 & 15,6 & 20,4 & 19,5 & 10000 & 23334 & 161.30 & 26 & 76 & 117 & 8.6 & 11,0 & 10,4 & $\cdots$ \\
\hline $\begin{array}{l}\text { 10. Restaurant/ } \\
\text { Cafe }\end{array}$ & 14 & 25 & 26,5 & 6,6 & 6,1 & 5.6 & 40 & 100 & 128 & 3,1 & 3,4 & 3,0 & 2857 & 1000 & 4866 & 12 & 25 & 35 & 3,7 & 3,6 & 3,1 & $\cdots$ \\
\hline surne & 213 & 411 & 483,5 & 100 & 100 & 100 & 1280 & 2940 & 4318 & 100 & 100 & 100 & 6009 & 7153 & 8932 & 303 & 689 & 1124 & 100 & 100 & 10 & ... \\
\hline
\end{tabular}

Tab. 13: Belsple1hafte varengruppenbezogene Strukturprojektion

Tab. 13: Hypothetisches Beispiel einer warengruppenbezogenen Strukturprojektion 
Das Beispiel zeigt zwar einerseits eine praktikable Vorgehensweise zur Uberprüfung der Zielwirkung von Marketingstrategien auf, andererseits werden jedoch auch die damit verbundenen Probleme deutlich sichtbar. Dies betrifft zum einen die i.d.R. subjektiven Schätzungen der zielerreichungsgrade und zum anderen die Zurechnung der Zielwirkung zu konkreten Marketingstrategien. Die formal zwar richtige ausschließliche Betrachtung der Verkaufsflächenstrategie ist unter materiellen Gesichtspunkten nicht haltbar, da unter einer Flächenausweitung ein ganzes Bündel von Marketing-Instrumentalstrategien subsummiert ist, die von Sortiments- über Plazierungs- bis hin zu Verkaufspersonalstrategien reichen. Insofern müssen diese globalen Analysen im Hinblick auf die Beurteilung der einzelnen isolierten Marketing-Instrumentalstrategien ergänzt werden. Hier sind Methoden heranzuziehen, die den Vergleich alternativer Marketingstrategien anhand einer oder mehrerer zielkriterien ermöglichen.

Für diese Fragestellungen können z.B. Punktbewertungsverfahren herangezogen werden. Die Tabelle 14 verdeutlicht den Grundgedanken an einem hypothetischen Beispiel aus der Distributionspolitik.

Die Warenhausleitung steht vor dem Problem, die Möglichkeiten der Spezialhausstrategie für eine Filialgruppe zu überprüfen. Die Einzelfilialen dieser Filialgruppe weisen alle für die Warengruppe $x$ ein sehr hohes, noch nicht ausgeschöpftes Marktpotential auf. Insofern stehen u.a. folgende strategische Alternativen zur Verfügung:

(1) Ausgliederung der Warengruppe X ( = konsequente Branching-out-Strategie)

(2) Sortimentsumschichtung auf bestehender Fläche , der Ausbau der ertragsstarken und Flächenreduzierung der ertragsschwächeren Atrikelgruppen

(3) Vergrößerung der Verkaufsfläche für die Warengruppe $\mathrm{X}$ innerhalb der Filialen durch Lagerverlagerung, andere Warenpräsentation, Flächenfreisetzung bei benachbarten Abteilungen. 


\begin{tabular}{|c|c|c|c|c|c|c|c|}
\hline \multirow[b]{2}{*}{ Kriterien } & \multirow[b]{2}{*}{ G } & \multicolumn{2}{|c|}{ A 1} & \multicolumn{2}{|c|}{ A 2} & \multicolumn{2}{|c|}{ A 3} \\
\hline & & Note & Wert & Note & Wert & Note & Wert \\
\hline $\begin{array}{l}1 \text { Marktpotential- } \\
\text { ausschöpfung }\end{array}$ & 15 & 5 & 75 & 1 & 15 & 3 & 45 \\
\hline 2 Umsatz & 25 & 5 & 100 & 2 & 50 & 3 & 75 \\
\hline 3 Marktanteil & 25 & 5 & 125 & 2 & 50 & 4 & 100 \\
\hline $\begin{array}{l}4 \text { Kosten } \\
\text { (Investitionen) }\end{array}$ & 10 & 1 & 10 & 5 & 50 & 4 & 30 \\
\hline $\begin{array}{l}5 \text { Deckungsbeitrag } \\
\text { (mittelfristig) }\end{array}$ & 10 & 1 & 10 & 5 & 50 & 3 & 30 \\
\hline $6 \begin{array}{l}\text { Rendite } \\
\text { (mittelfristig) }\end{array}$ & 15 & 1 & 15 & 5 & 75 & 3 & 45 \\
\hline Summe & & & 360 & & 290 & & 335 \\
\hline Rangplatz & & & 1. & & 3. & & 2. \\
\hline
\end{tabular}

Tab. 14 : Beispiel für ein Punktbewertungsverfahren zur Bewertung und Auswahl von Strategien

Die Bewertung der Alternativen ergibt eine Bevorzugung der konsequenten Branching-out-Strategie. Diese erste Beurteilung, die sich im Grunde an den "Marktchancen" orientiert, muß nun vor ihrer Realisierung noch mit einer Reihe zusätzlicher Analysen abgesichert werden. Insbesondere ist das Risiko der Strategien genauer zu erfassen. So können z.B. Imageverluste durch die Ausgliederung der attraktiven Warengruppe $x$ auftreten, Verbundeffekte wegfallen, die Kundenfrequenz sinken usw., die eine Relativierung der Beurteilung notwendig machen und evtl. eine andere strategiealternative realisierungswürdiger erscheinen lassen.

Ungeachtet dieser Erweiterungsmöglichkeiter bieten Punktbewertungsverfahren die Möglichkeit zur einfachen Grobbewertung von Marketingstrategien. Der Aussagewert der solchermaßen getroffenen Entscheidungen muß allerdings in realen Planungssituationen aufgrund der bereits angesprochenen Kritikpunkte der notwendigen mehrfach subjektiven Schätzung, der zugrundeliegenden Verknüpfungsregel 
sowie der fehlenden Annahme- oder Ablehnungsschranken jeweils kritisch hinterfragt werden. Insofern kann diese Methode lediglich erste grobe Hinweise auf die Vorzugswürdigkeit von strategiealternativen geben.

Aufgrund der berechtigten Kritikpunkte an solchen einfachen Entscheidungsverfahren wird häufig versucht, Detailprobleme im Rahmen der strategischen Feinplanung mittels' anspruchsvoller analytischer Methoden zu lösen. So wird $z . B$. von Brauer der Vorschlag entwickelt, die optimale Warengruppenstruktur mit der linearen Programmierung zu ermitteln ${ }^{1}$. Er formuliert einen linearen Programmierungsansatz für drei warengruppen und wählt als zielgröße die Gesamtleistung, die er als abhängige Größe vom Umsatz und den Arbeitsstunden definiert. Als relevante Restriktionen berücksichtigt er z.B. sinnvolle Ober- und Untergrenzen für die erzielbaren Umsätze in den einzelnen Abteilungen. Weitere Varianten dieses Grundansatzes gehen z.B. vom Deckungsbeitrag als Zielgröße aus und berücksichtigen eine Vielzahl weiterer Restriktionen ${ }^{2}$. Damit ist ein Modell strukturiert, das eine im Hinblick auf die gewählte zielgröße optimale Warengruppenstruktur ermittelt. Für die praktische Anwendung bei realen strategischen Problemstellungen der Warenhäuser erscheinen diese Vorschläge jedoch kaum geeignet, da ihre Anwendung mit einer Vielzahl von Prämissen verknüpft ist, die nicht erfüllbạ erscheinen. So muß z.B. die Abhängigkeit der Gewinngröße von beeinflußbaren Faktoren bekannt und linear sein (additive Verknüpfung). Während die lineare Abhängigkeit lediglich einen formalen

1 Vgl. hierzu Brauer, K., Die Ermittlung der optimalen Warengruppenstruktur einer Filiale in Lebensmittelfilialbetrieben, in: Grümbel, R., et al., Unternehmensforschung im Handel, Rüschlikon-Zürich 1969, S. 53-124.

2 Auf eine detaillierte Beschreibung der mathematischen Struktur wird hier verzichtet. Vgl. dazu Brauer, K., a.a.O., S. $67 \mathrm{ff.}$ 
Einwand darstellt, ist die Hauptkritik gegen die Kenntnis der exakten Abhängigkeiten zwischen unterschiedlichen Parametern zu richten. Sie setzt im Grunde die Kenntnis der Marktreaktionsfunktion voraus - ein wie zuvor bereits erwähnt-bislang ungelöstes theoretisches Problem. Damit scheitert der Einsatz dieser Methode als globales Planungskonzept an der fehlenden Informationsvoraussetzung. Dies bedeutet allerdings nicht, daß dieser Vorschlag wie vom Autor auch primär beabsichtigt - nicht für partielle Probleme im Rahmen der strategischen Feinplanung Einsatz finden kann. Dies gilt auch für eine Vielzahl weiterer analytischer Planungs- und Entscheidungsmethoden, die sich z.B. mit dem Problem der Standortwahl ${ }^{1}$, der Warenpräsentation $^{2}$, der Mediaselektion ${ }^{3}$, der Werbebudgetierung ${ }^{4}$ usw. befassen. Sie können wesentlich dazu beitragen, den Einsatz der Marketinginstrumente in Warenhäusern bei isolierter, disaggregierter Betrachtungsweise $\mathrm{zu}$ planen und somit die Zielerreichung innerhalb des durch die globalen Planungsmethoden - wie z.B. die Portfolio-Methode vorgebenenen Rahmens sicherzustellen.

Aufgrund ihres partiellen Charakters können diese Ansätze nur begrenzt dazu beitragen, die einzelnen Marketinginstrumente zu einem sinnvollen, d.h. zieladäquaten Marketingmix zu integrieren. Die Warenhausleitung steht vor der

1 Vgl. hierzu z.B. Tietz, B., Standort- und Geschäftsflächenplanung, a.a.O., insbesondere S. $173 \mathrm{ff.}$.

2 Vgl. hierzu z.B. den tberblick bei Barth, K., Warenpräsentation, a.a.O., S. 95 ff sowie die dort angegebene Literatur.

3 Vgl. hierzu z.B. die modellanalytischen Ansätze in: Freter, H., Media-Selektion, Informationsgewinnung und Entscheidungsmodelle für die Werbeträgerauswahl, Wiesbaden 1974 .

4 Vgl. hierzu den überblick bei Meffert, H., Marketing, a.a.O., S. $427 \mathrm{ff}$. sowie die dort angegebene Literatur. 
Aufgabe, die systematisch abgeleiteten - auf sukzessiv rückgekoppelten Planungsschritten beruhenden - MarketingInstrumentalstrategien $z \mathrm{u}$ einem abgestimmten strategischen Marketing-Mix zu kombinieren. Die grundsätzliche Problematik der Kombination von Marketingstrategien läßt sich mit der Darstellung eines "Spinnennetzes" verdeutlichen: Unabhängig von einer spezifischen Unternehmenssituation läßt sich das warenhausadäquate Aktivitätsniveau für die zentralen Marketinginstrumente unter Berücksichtigung der unterschiedenen idealtypischen Basisstrategien wie in Abbildung 27 darstellen ${ }^{1}$.

Die Form des Spinnennetzes veranschaulicht dabei das $\mathrm{Zu-}$ sammenwirken der einzelnen Marketinginstrumente: es ist je nach Basisstrategie enger oder weiter gefaßt. Grundsätzlich zeigt die skizzierte qualitative Einschätzung der Abbildung 27 die Tendenz der Warenhäuser zu einem relativ weit gespannten Netz. Das damit verbundene relativ hohe Aktivitätsniveau in nahezu allen Instrumentebereichen ist das Kennzeichen und die Stärke der Warenhäuser. Hierdurch gelingt eine deutliche Abhebung und Profilierung gegenüber jenen Betriebsformen, die sich lediglich auf eine oder wenige Dimensionen konzentrieren (z.B. Discounter: primär Preislage). Unter der Prämisse, daß die Verbraucher profilierende Anstrengungen in nahezu allen Instrumentebereichen auch honorieren, gewährt dieses breite und zugleich hohe Aktivitätsniveau die Chance, möglichst viele Verbraucher "einzufangen" 2 , was nicht zuletzt in der breiten Zielgruppe der Warenhäuser zum Ausdruck kommt.

1 Diese Abbildung dient lediglich der Veranschaulichung des Grundgedankens, daß verschiedene Betriebsformen in den Marketinginstrumenten unterschiedliche Aktivitätsniveaus realisieren. Die konkrete empirische Fundierung scheitert an der Unterschiedlichkeit der Messung der einzelnen Aktivitätenausprägung, so daß dieser Abbildung lediglich eine plausibel-strukturierende Funktion zukommt.

2 Vgl. zu diesem Gedanken: Steffenhagen, H., Marktführer, a.a.0., s. 28 . 


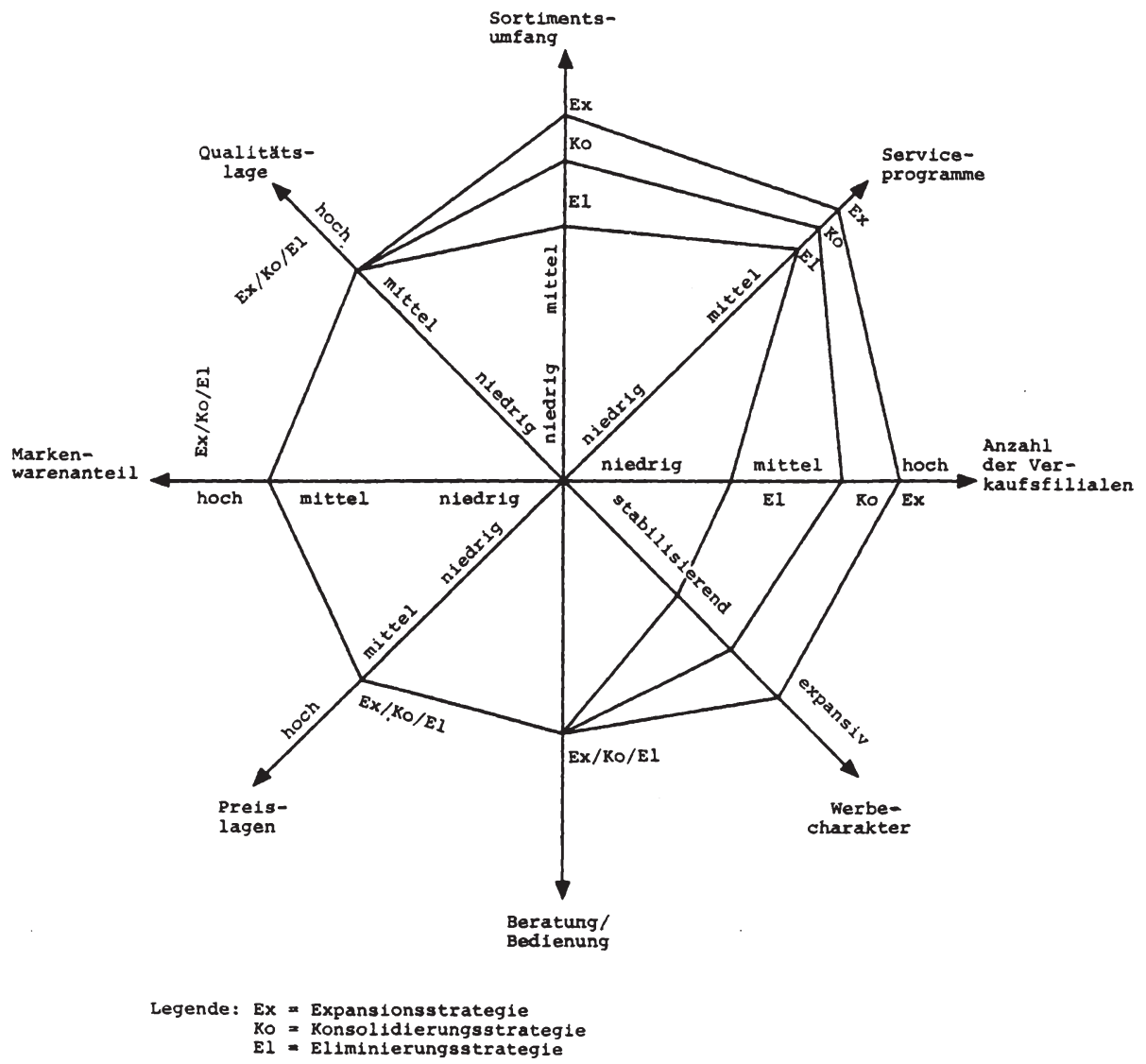

Abb. 27 : Aktivitätenniveau(Netzstrategie) der Marketinginstrumente in Warenhäusern 
Die grundsätzliche Problematik bei der Ableitung von Marketingstrategien für den einzelnen Aktionsparameter besteht in den vielfältigen vertikalen und horizontalen Interdependenzen ${ }^{1}$, die in Abbildung 27 durch die verschiedenen "Spinnenfäden" zum Ausdruck kommen. Die vertikalen Interdependenzen ${ }^{2}$ beinhalten die $\mathrm{Ab}-$ hängigkeiten zwischen der Festlegung der Basisstrategien und der Festlegung der Marketing-Instrumentalstrategien. Sie finden einerseits in der gewählten Reihenfolge der verschiedenen Planungsstufen sowie andererseits in dem iterativen Charakter des gesamten Planungsprozesses Berücksichtigung ${ }^{3}$.

Die horizontalen Interdependenzen umfassen die vielfältigen, wechselseitigen Abhängigkeiten zwischen den Ausprägungsniveaus (z.B. Bedienungsform-Qualitätsniveau - Preislage Warenpräsentation - Persönlicher Verkauf) der einzelnen Marketinginsturmente. Deshalb muß die Warenhausleitung bereits bei der isolierten Festlegung jedes einzelnen Instrumenteniveaus die möglichen Auswirkungen auf die übrigen Marketinginstrumente mit berücksichtigen, um insgesamt die zielerreichung sicherzustellen. Damit folgt der isolierten Betrachtung der Marketinginstrumente

$1 \mathrm{Vgl.} \mathrm{zum} \mathrm{Problemkreis} \mathrm{der} \mathrm{Interdependenz} \mathrm{zwischen}$ Marketing-Instrumenten z.B. Köhler, R., Marketing-Entscheidungen als Anwendungsgebiet der quantitativen Planung, in: Köhler, R., zimmermann, H.-J. (Hrsg.), Entscheidungshilfen im Marketing, stuttgart 1977, S. 2-28, hier S. 3 ff.; Kühn, R., Marketing-Mix, in: Poth, L.G. (Hrsg.), Marketing 3, Neuwied 1979, S. 10 ff.; Meffert, H., Marketing, a.a.O., S. 484 .

$2 \mathrm{Vgl.} \mathrm{zum} \mathrm{Begriff} \mathrm{der} \mathrm{vertikalen} \mathrm{Interdependenzen:}$ Wild, J., Grundlagen, a.a.O., S. 189.

3 Vgl. hierzu Gliederungspunkt 5.1 und Abbildung 27. 
das Problem der zieladäquaten strategischen Kombination der einzelnen Aktionsparameter ${ }^{1}$.

Aufgrund der Vielfalt von Kombinationsmöglichkeiten im Marketinginstrumentarium, der Unsicherheit der Marktreaktion bzw. der Wirkungsprognose bei alternativen Aktivitätsniveaus sind bis heute noch keine methodischen Ansätze entwickelt, die dieses problem befriedigend lösen können ${ }^{2}$. Vielversprechend erscheinen allerdings

1 Damit ist im Kern das Problem der Bestimmung des Marketing-Mix angesprochen. Vgl. hierzu als grundlegende Literatur: Balachandran, V., Gensch, D.H., Lösung des Marketing-Mix-Problems mit Hilfe der geometrischen Programmierung, in: Köhler, R., Zimmermann, H.-J., (Hrsg.), Entscheidungshilfen im Marketing, stuttgart 1977, S. 449-469; Kotler, P.. Marketing-Management, a.a.O., S. 251 ff.; Kühn, R., a.a.O., S. 3 ff.; Lambin, S.-J., Ein On-Line-Modell zur computergestützten Planung des Marketing-Mix, in: Köhler, R., Zimmermann, H.-J. (Hrsg.), Entscheidungshilfen im Marketing, a.a.O., Stuttgart 1977, S. 430-447; Meffert, H., Marketing, a.a.O., S. 476 ff.; derselbe, Zum Problem des Marketing-Mix. Eine heuristische Vorauswahl absatzpolitischer Instrumente, in: Marketing heute und morgen, Hrsg. H. Meffert, Wiesbaden 1975, S. 257-275;

Seeringer, W., Ein Ansatz zur Lösung des Marketing-MixProblems, in: JdAV, 1977, S. 143-160; Thummel, D., Entwicklung einer Konzeption zur Bestimmung des langfristig-strategischen Marketing-Mix, Bern und Stuttgart 1972; Topritzhofer, E., Marketing-Mix, in: Handwörterbuch der Absatzwirtschaft, hrsg. v. Tietz, B., Stuttgart 1977, Sp. 1247-1264.

2 Insbesondere analytische Methoden, die durch eine simultane Betrachtung der Marketinginstrumente eine optimale Lösung des Problems anstreben, erweisen sich aufgrund der einschränkenden Prämissen (Informationsbedarf, Lösungsalgorithmen usw.) für die praktische Anwendung als irrelevant. Vgl. hierzu den Uberblick bei Meffert, H., Marketing, a.a.O., S. 493 ff.; Thumel, D., a.a.O., S. 14 ff.; Topritzhofer, E., Zur programmatischen Brauchbarkeit marginal-analytischer Marketing-Mix-Modelle, in: Köhler, R., Zimmermann, H. -J. (Hrsg.), Entscheidungshilfen im Marketing, Stuttgart 1977, S. 395-413. 
heuristische Methoden, die mit Hilfe von Verfahrensregeln suboptimale Lösungen anstreben ${ }^{1}$. Wie sie bei der Ableitung der zieladäquaten Marketing-Instrumentalstrategien in Warenhäusern zum Einsatz kommen, strukturiert die Abbildung 28 . Demnach bildet die Analyse der gegenwärtig realisierten Kombination der Marketing-Instrumentalstrategien eines Warenhauses den Ausgangspunkt der Planungsbemühungen ${ }^{2}$.

Die realisierte Kombination läßt sich zunächst als unmittelbare Konsequenz der mit Hilfe der Portfolio-Methode entwickelten Marketing-Basisstrategien begreifen. Zusätzlich werden sie von noch nicht berücksichtigten situationalen Faktoren und Marketing-zielen beeinfluBt. Diese Größen bilden gleichzeitig die Grundlage zur Uberprüfung sowohl der einzelnen Marketing-Instrumentalstrategien als auch ihrer realisierten Kombination. Im Einzelfall muB überprüft werden, ob

- die Basisstrategien und Marketing-Instrumentalstrategien kompatibel sind

- die logische Konsistenz der Marketing-Instrumentalstrategien im Hinblick auf die sachlichen und zeitlichen Interdependenzen gewährleistet ist und

- sich die Aktivitätsniveaus mit den strategischen Zielsetzungen des Warenhauses vereinbaren lassen.

Als Ergebnis dieser Beurteilungsphase liegen die Konfliktbereiche der Instrumentalkombination des Warenhauses offen.

1 Vgl. den Uberblick bei Kühn, R., a.a.O., S. 28 ff.; Meffert, H., Marketing, a.a.O., S. 487 ff.; Thummel, D., a.a.O., S. 55 ff..

2 Damit wird von der realistischen Prämisse einer bestehenden Warenhausunternehmung, die den Markt mit spezifischen Marketing-Instrumentalstrategien bearbeitet, ausgegangen. 


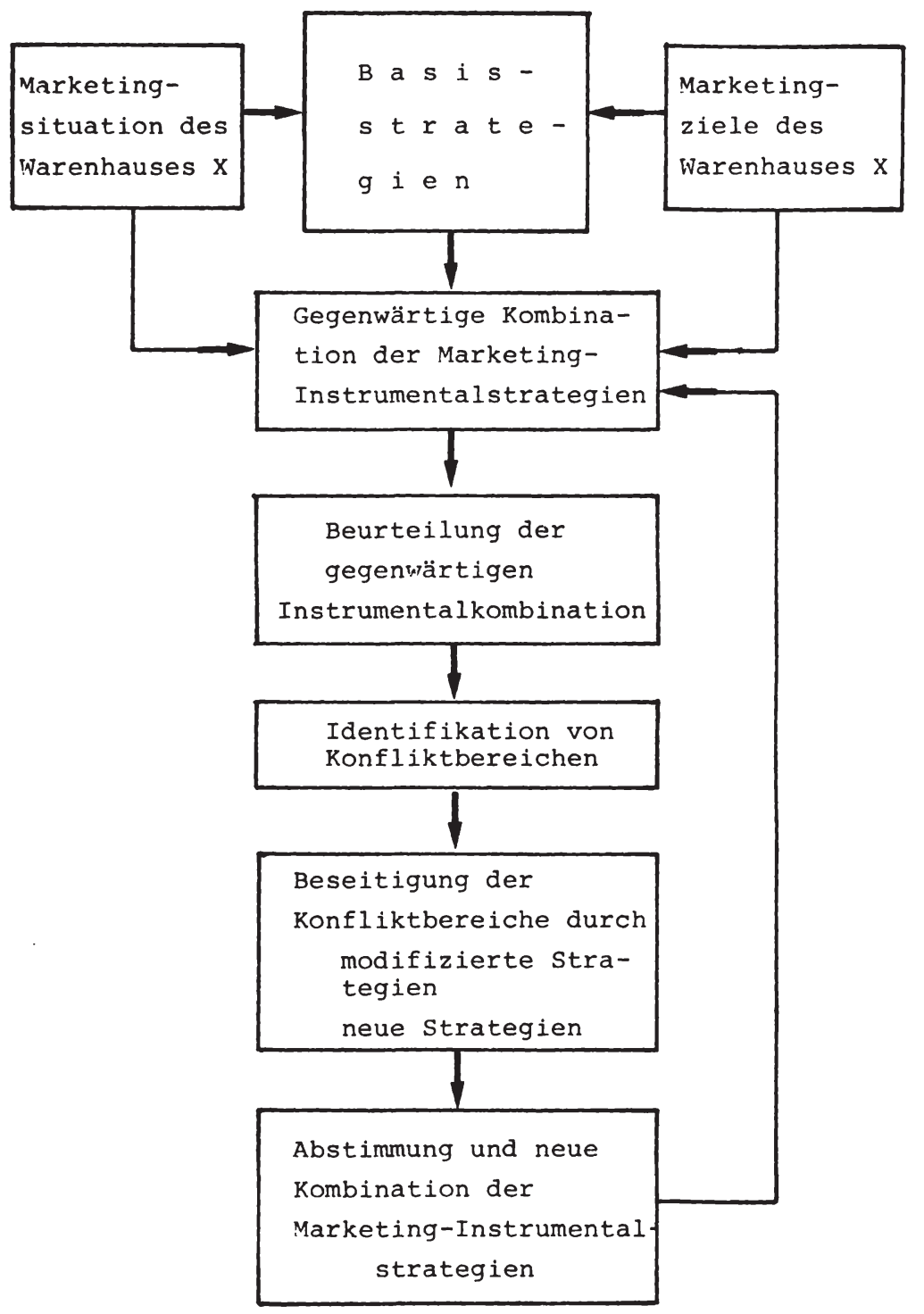

Abb. 28: Heuristische Vorgehensweise zur Kombination der Marketing-Instrumentalstrategien in Warenhäusern 
Folgerichtig wird die Warenhausleitung in der anschließenden Kreativitätsphase die erkannten Konfliktbereiche $z u$ beseitigen suchen. In der realen Planungssituation kommt normalerweise ein abgestuftes Vorgehen zum $\mathrm{Zuge.}$ Zunächst wird versucht, die Konsistenz bzw. Zieladäquanz durch die Modifikation aktueller Strategien $z u$ erreichen. Dabei bietet es sich entsprechend der hier vorgeschlagenen Planungsfolge ${ }^{1}$ an, zunächst die sortiments- und distributionspolitischen Strategien festzulegen sowie untereinander abzustimmen, um hierdurch die Basis für die Festlegung der übrigen Instrumentebereiche $z u$ erhalten. Nur für den Fall, daß die Schwachstellenermittlung gravierende Abweichungen aufzeigt, wird die Warenhausleitung unter Zuhilfenahme des gesamten methodischen Instrumentariums der Strategieplanung neue Strategien generieren. Die zweifellos schwierige Abstimmung und erneute Zusammenfügung zu einer situations- und zieladäquaten Kombination stellt sicher, daß durch die modifizierten bzw. neuen Strategien die Zielerreichungsgrade der beibehaltenen Strategien nicht negativ beeinflußt werden. Den AbschluB dieses mehrfach rückgekoppelten Abstimmungsund Anpassungsprozesses bildet die Integration zur neuen - gegenüber der Ausgangslage verbesserten - strategischen Kombination der Marketinginstrumente.

Diese einfache heuristische Vorgehensweise kann nur als grobe Richtschnur für die Verbesserung der Kombination der Marketing-Instrumentalstrategien in Warenhäusern angesehen werden. Sie beschreibt letztlich nur den prozessualen Charakter der sukzessiven Veränderung einer bestehenden Marketing-Instrumentekombination, die durch mehrfache Strategieanpassung eine bessere Zielerreichung sicherstellen soll. Bei allen Bedenken, die gegen eine solche, vereinfachte Struktur eines Lösungsansatzes

1 Vgl. Kapitel 5.1 dieser Arbeit. 
vorgebracht werden können, muß berücksichtigt werden, daß auf der hier betrachteten, durch große Komplexität gekennzeichneten Planungsebene nur eine "Politik der kleinen Schritte" weiterbringt. Diese Vorgehensweise erscheint so lange erfolgversprechend, wie der methodische Erkenntnisfortschritt der Theorie die Entwicklung eines Totalmodells, das simultan das strategische Ausprägungsniveau aller Marketinginstrumente zieladäquat festlegt, noch nicht ermöglicht.

Die erfolgreiche Durchführung dieses notwendigen mehrstufigen Abstimmungsprozesses setzt ein adäquates organisationssystem $^{1}$ voraus. Dieses muß als Kern sowohl eine vertikale Integration der auf unterschiedlichen hierarchischen Ebenen entwickelten Marketingstrategien ermöglichen als auch die horizontale Koordination der auf hierarchisch gleichrangigen stufen entwickelten Marketingstrategien für die beiden Bereiche "Ware" und "Filiale" sicherstellen.

1 Das Organisationssystem umfaßt sowohl die organisationsstruktur als auch den Ablauf der organisatorischen Prozesse, die sich beide an dem vorgegebenen Planungssystem orientieren müssen. 
6. Implikationen für die Durchsetzung der strategischen Marketingplanung in Warenhäusern

6.1 Ansätze zur Koordination und Integration der Marketingstrategien

Die aktuell realisierten Planungssysteme von Warenhäusern stellen in der Regel die gesamtunternehmensbezogene Sichtweise der Zentrale in den Vordergrund. Dementsprechend orientiert sich die strategische Marketingplanung an den durchschnittlichen Merkmalen und Erfolgskennziffern aller Waren- und Filialgruppen. Diese Vorgehensweise erklärt sich aus der früheren - in einer Verkäufermarktsituation' ${ }^{1}$ sinnvollen - einkaufsorientierten Denkweise. Im Hinblick auf die gegenwärtige Käufermarktsituation und ihre notwendige Kundenorientierung ist bei dieser organisationsstruktur insbesondere die Vernachlässigung der verkaufsstrategischen Ausrichtung in den einzelnen Filialgruppen und Filialen problematisch. Dies impliziert, daß das gegenwärtige Planungs- und damit das Organisationssystem ${ }^{2}$ der Warenhäuser im Hinblick auf eine stärkere Beteiligung der dezentralen Organisationseinheiten bei der strategischen Marketingplanung entwickelt

1 Vgl. Meffert, H., Marketing, a.a.O., S. 34.

2 Dabei werden die Auswirkungen der Planungssystematik auf die Aufbauorganisation der Warenhäuser weitgehend vernachlässigt. Vgl. hierzu den auch heute noch aktuellen überblick über alternative Ansätze sowie ihre kritische Diskussion bei Hanhart, E.W., Marktgerechte Koordination von Einkauf und Verkauf im Warenhaus, Bern 1967. Gute praxisorientierte Darstellungen geben Berger, R., Organisation der Unternehmensführung, in: Der Filialbetrieb als System - Das Cornelius-StüssgenModell, hrsg. v. Nieschlag, R., Eckardstein, D., Köln 1972, S. 91-145; Bruckhaus, M., a.a.O., S. $123 \mathrm{ff} .$. 
werden muß. Ein solchermaßen neu entwickeltes Planungsund Organisationssystem muß einer Reihe von Anforderungen genügen, die Meffert mit "Integration", "Flexibilität", "Kreativitätsförderung", "Spezialisierung", "Effizienz" umschreibt". Auf der Basis dieser Anforderungskriterien sind im folgenden die im Zusammenhang mit Warenhäusern diskutierten idealtypischen Planungsverfahren zu analysieren.

\subsection{Darstellung und kritische würdigung idealtypischer Planungsverfahren in Warenhäusern}

Idealtypischerweise kann die Entwicklung und Durchsetzung von Marketingstrategien im Warenhaus über verschiedene Hierarchieebenen hinweg in zwei Richtungen verlaufen: Von oben nach unten (rein zentrales Planungssystem = "top-to-down"-planung) oder von unten nach oben (rein dezentrales $\mathrm{Planungssystem} \mathrm{=} \mathrm{"bottom-up"-Planung}{ }^{2}$ ).

Bei der "top-to-down"-Planung in Warenhäusern werden die Marketingstrategien ausschlieblich von der zentralen Führungsspitze bis ins Detail erarbeitet. Die nachgelagerten Planungsebenen haben diese Strategien als Datum zu akzeptieron und lediglich die Kompetenz, die Strategien zu konkretisieren, die Maßnahmenpläne zu erstellen und

1 Vgl. Meffert, H., Marketing, a.a.O., s. $508 \mathrm{ff.}$.

2 Vgl. zur Darstellung und kritischen Würdigung dieser beiden Verfahren z.B. Aghte, K., Strategie und Wachstum, a.a.O., S. 93 ff.; Eckhardt, A., Strategien der organisatorischen Gestaltung, Frankfurt 1979, S. 74 ff.; Teichmann, H., Planungsprobleme der Zentralen im dezentralisierten Unternehmen und Lösungsmöglichkeiten für die Praxis, in: DBW 39 (1979), Heft 3, S. 377-395, hier S. 377 f.; Wild, J., Grundlagen, a.a.O., S. 189 ff.. 
den nachgelagerten Planungsstellen vorzuschreiben. Gegen diese Vorgehensweise spricht insbesondere die ungenügende Berücksichtigung des spezifischen Wissens und der Erfahrungen nachgelagerter Planungsstufen ${ }^{1}$. Darüber hinaus hat die hohe Zentralisationswirkung negative Auswirkungen auf die Motivation und Zufriedenheit der nachgelagerten Planungsinstanzen. Nicht zuletzt wird durch die strenge hierarchische Ordnung die Flexibilität im Planungssystem stark eingeschränkt.

In dieser Vorgehensweise stellt die "bottom-up"-Planung die Antithese dar. Die Planung beginnt mit der untersten noch an der strategischen Marketingplanung beteiligten Ebene. Sie entwickelt spezifische disaggregierte Strategien und reicht diese an die nächste übergeordnete Instanz weiter. Diese faßt die Substrategien zusammen, koordiniert und integriert sie zu einem konsistenten Strategiensystem und reicht sie ihrerseits weiter.

Gegen diese Planungsweise spricht insbesondere die fehlende Ausrichtung auf übergeordnete, gesamtunternehmensbezogene (ziele bzw.) Strategien. Hierdurch entsteht die Gefahr, daß die auf disaggregierter Ebene entwickelten Strategien untereinander unverträglich sind und nicht auf ein gemeinsames, übergeordnetes ziel hinführen. Dies erfordert entweder eine sogenannte negative Koordination (= Anpassung an das unterste gemeinsame Zielniveau) oder eine Neuplanung, die von den übergeordneten Ziel- und Strategievorstellungen des Topmanagement ausgeht und nach unten fortschreitend sämtliche Teilpläne entsprechend uberarbeitet und erneut koordiniert ${ }^{2}$. Hinzu kommt das Problem, daß das - der Planung von Strategien immanente Konfliktpotential auf einem vergleichsweise breiten Feld

1 Vgl. Wild, J., Grundlagen, a.a.0., S.189 f..

2 Vgl. ebenda, S. $194 \mathrm{f}$.. 
ausgetragen werden muß, auf dem zudem die Mechanismen der Konfliktaustragung weniger stark ausgearbeitet sind als innerhalb der Geschäftsleitung" . Im Vergleich zur "top-todown"-Planung werden bei dieser Vorgehensweise aber die Innovationsbereitschaft, Motivation und Spezialisierung der Planungsbeteiligten stärker berücksichtigt.

Insgesamt sind damit beide Planungsverfahren in ihrer idealtypischen Form nicht verwendbar, da sie das Problem der vertikalen Interdependenzen nicht zufriedenstellend lösen können. Denn für beide Fälle gilt, daß die untergeordneten (ziele und) Strategien nicht ohne Kenntnis der übergeordneten (ziele und) Strategien festgelegt werden können und umgekehrt ${ }^{2}$. Daraus ergibt sich die Notwendigkeit, beide Vorgehensweisen zu vereinen. Dazu sind zunächst die (Ausgangsziele bzw.) Ausgangsstrategien in einem Proze $\beta$ der "top-do-down"-Planung herunterzubrechen, um dann anschließend in einer aufwärts gerichteten "bottom-up"-Planung die konkreten Strategien als realisierbar zu bestätigen und verbindlich festzuschreiben. Dieses Vorgehen entspricht der Planung nach dem Gegenstromverfahren $^{3}$ ("down-up-Planung").

Die konkrete Ausgestaltung dieses Planungsverfahrens für die hierarchisch-dualistische strategische Marketingplanung in Warenhäusern wird im folgenden für die drei Planungsebenen Warenhausleitung, SGE-Leitung (Warengruppenund Filialgruppenleitung) und Subgruppenleitung (Artikelgruppen- und Einzelfilialleiter) beschrieben ${ }^{4}$.

1 Vgl. Eckhardt, A., a.a.0., s. 80.

2 Wild spricht hier vom "logischen Zirkelproblem". Vgl. Wild, J., Grundlagen, a.a.O., S. 195.

3 Diesen Begriff entwickelte Wild. Vgl. Wild, J., Grundlagen, a.a.O., S. $196 \mathrm{ff}$..

4 Die Beschränkung auf diese drei hierarchischen Ebenen ist ausreichend, da nur sie echte strategische Problemstellungen zu lösen haben, wobei dies bereits für die dritte Ebene nur einschränkend gilt. 
6.13 Planung von Marketingstrategien in Warenhäusern nach dem Gegenstromverfahren

Ausgangspunkt des Prozesses der Ableitung von Marketingstrategien bilden die planerischen Aktivitäten des Vorstandes (vgl. Abbildung 29). Aufgrund seiner primären Aufgaben, dem Management der Warengruppen sowie dem Management der Filialgruppen, legt er zusammen mit dem zentralen Planungsstab die Rahmenentscheidungen fest und entwickelt die Basisstrategien sowie die groben Richtlinien für die darauf aufbauenden Marketing-Instrumentalstrategien. Hierbei werden die Warengruppen- und Filialgruppenleiter beratend hinzugezogen.

Die von der Warenhausleitung entwickelten Strategien sind einerseits bewußt so vage gehalten, daß der motivations- und kreativitätsfördernde Freiraum für die nachgelagerte Planung der SGE-Leitung gewährleistet ist. Andererseits erfüllen sie die notwendige richtungsweisende Funktion, da sie die Strategiealternativen auf der SGEEbene einengen. Hinzu tritt als motivationsförderndes Element die zumindest partielle Mitwirkung nachgelagerter Planungsinstanzen bei cier Strategieentwicklung, die die Identifikation mit den Basisstrategien vergrößert und ihr Spezialwissen nutzt.

Die auf diese Weise festgelegten vorläufigen Strategien werden nun der Leitung der strategischen Geschäftseinheiten vorgegeben. Dabei erfolgt zunächst eine getrennte Vorgehensweise für die strategische Warengruppenplanung und die strategische Filialgruppenplanung.

Die Aufgabe der Warengruppenleitung ist die Ausgestaltung der Marketing-Instrumentalstrategien und speziell der Sortiments- und Preispolitik. Hierbei sind die Breite and 


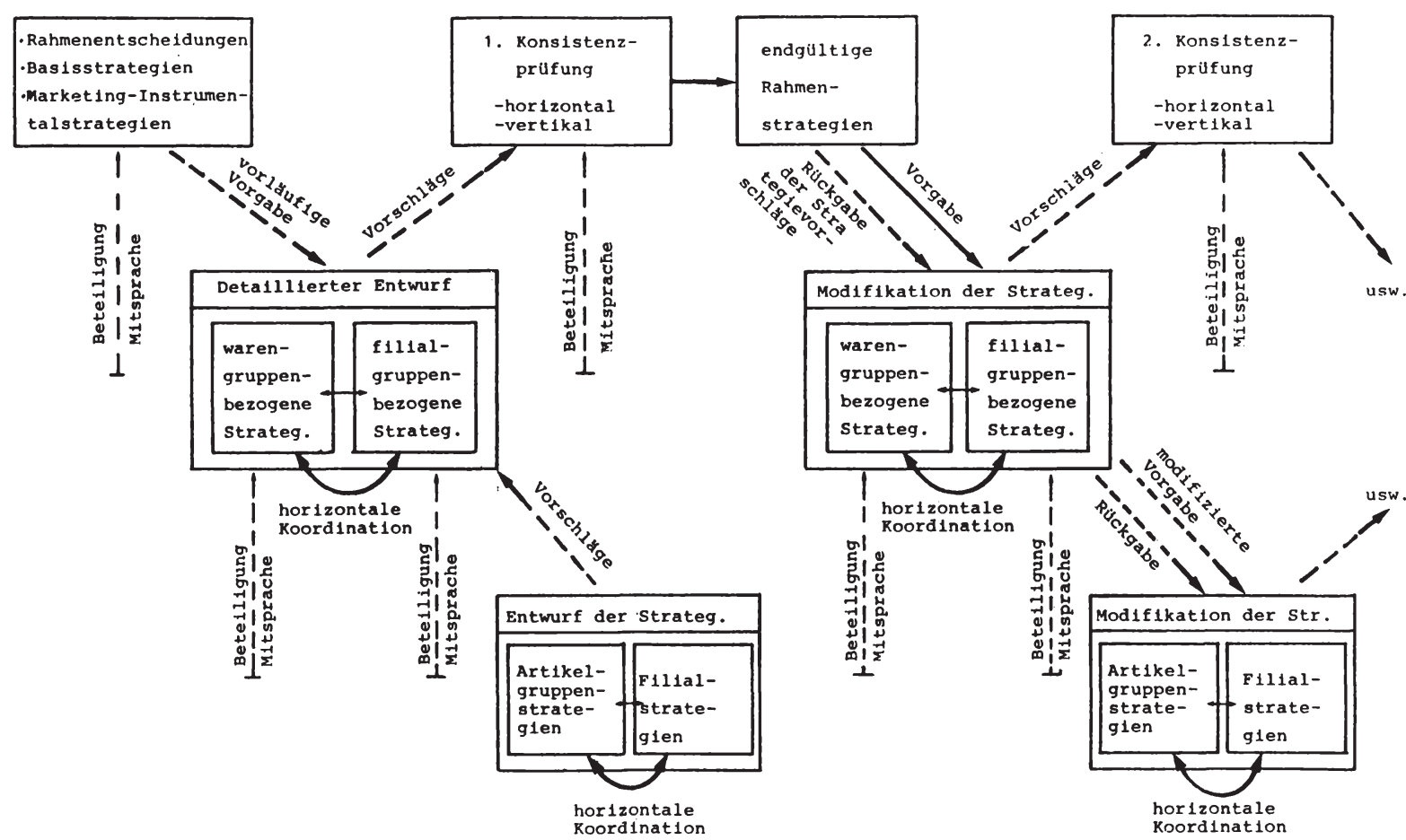

Abb. 29: Strategienplanung in Warenhäusern nach dem Gegenstromverfahren 
Tiefe jeder Warengruppe exakt umschrieben. Dies betrifft zum einen die Struktur bzw. Gewichtung der Artikelgruppen sowie die Entscheidungen über die Qualitätslage und die Markenpolitik. Diese Entscheidungen determinieren unmittelbar die Preislagen- sowie die Preisspreizungsstrategie innerhalb der einzelnen Artikelgruppen. Ferner ist $\mathrm{zu}$ entscheiden, welche Artikelgruppen kommunikativ besondere Aufmerksamkeit erhalten müssen, wovon sowohl die Werbestrategien als auch das Bedienungssystem sowie die Warenrpäsentation betroffen sind. Darüber hinaus sind eventuelle Besonderheiten in der Zielgruppen- und Konkurrenzstrategie zu berücksichtigen. So wird in der Regel warengruppenspezifisch eine Schwerpunktbildung bei subsegmenten der Basiszielgruppe erfolgen oder im Einzelfall auch eine außerhalb liegende Zielgruppe angesprochen werden. Bei diesen strategischen Entscheidungen muß die Warengruppenleitung insbesondere die Erfahrungen und Informationen der nachgelagerten Ebene, der Artikelgruppenleiter, berücksichtigen, denen entsprechende Beratungs- und Mitwirkungsrechte einzuräumen sind ${ }^{1}$.

Parallel zur strategischen Marketingplanung der Warengruppenleitungen müssen die Filialgruppenleitungen die distributions- und sortimentspolitischen Instrumentalstrategien spezifizieren.

Im Rahmen der distributionspolitischen Strategien erarbeiten die Filialgruppenleiter Vorschläge für Veränderungen innerhalb ihrer Filialgruppen, z.B. bezüglich baulicher Veränderungen, Neueröffnungen, Schließungen sowie

1 Diese Ebene wird im folgenden nicht weiter berücksichtigt, da sie keine eigenständige strategische Aufgabe zu erfüllen hat, sondern lediglich an den vorgelagerten Entscheidungen mitwirkt. 
Art und Ausstattung bei einzelnen Filialen. Dabei stützen sie sich auf die Erfahrungen und Informationen der beratend an der Planung mitwirkenden (Einzel-) Filialleiter. Auf der exakten Analyse der filialgruppenspezifischen Situation, die als zusammenfassung gleicher bzw. ähnlicher Jokaler Gegebenheiten interpretiert werden kann ${ }^{1}$, nimmt die sortimentspolitische Planung insbesondere auf standortspezifische zielgruppen- und konkurrenzbezogene Besonderheiten Bezug . Ausgehend vom "Idealsortiment", das durch die Warengruppenleitung erarbeitet wurde, wird ein filialgruppenspezifisches Sortiment zusammengestellt, das in der Regel vom Idealsortiment abweicht. Dies erfordert eine horizontale Abstimmung, d.h. die Koordination zwischen der Warengruppenleitung und der Filialgruppenleitung ${ }^{2}$. Als Ergebnis des Koordinationsprozesses liegen die Sortimentsstrategien der Filialgruppen fest, die zusammen mit den Distributionsstrategien die preis- und kommunikationspolitischen strategien determinieren. Die standortspezifische Situation der einzelnen Filialen erfordert insbesondere für die Kommunikationspolitik differenzierte Strategien, deren Konsistenz im Hinblick auf die Basis- und Instrumentalstrategien der Gesamtunternehmung noch überprüft werden muß.

Nach dem Durchlaufen dieses ersten Planungszyklus liegen horizontal abgestimmte SGE-Strategien vor, die nun an den Vorstand zurückgegeben werden. Gleichzeitig werden die vorläufigen SGE-Pläne an die Subgruppenleiter (Artikelgruppen- und Einzelfilialleiter) weitergeleitet, damit

$1 \mathrm{Vgl}$. die Überlegungen zur Filialgruppenbildung in Kapitel 2.21 der Arbeit.

2 Da diese Koordination ein zentrales Problem der planungsgerechten Organisation von Warenhäusern darstellt, wird es im nachfolgenden Kapitel gesondert behandelt. 
diese auf dieser Basis ihre vorläufigen Marketing-MaBnahmenpläne erstellen können ${ }^{1}$.

Der Vorstand hat die Konsistenz der SGE-Strategien in zweierlei Hinsicht zu überprüfen, einerseits - insbesondere im Konfliktfall - im Hinblick auf die horizontale Abstimmung der Waren- und Filialgruppenstrategien und andererseits im Hinblick auf die Integration der SGEStrategien in die gesamtunternehmensbezogenen Strategien. Im Falle der vollständigen horizontalen und vertikalen Konsistenz sind die SGE-Strategien untereinander kompatibel und in die übergeordneten strategien integriert. Dies bedeutet gleichzeitig das Ende des strategischen Planungsprozesses; die Strategien gelten als verabschiedet,werden den SGE-Leitungen zurückgegeben und bilden die Basis für die nachgelagerte operative und taktische Planung.

Die Komplexität des Problems der Planung von Marketingstrategien in Warenhäusern läBt jedoch diesen Fall als unrealistisch erscheinen. I.d.R. werden sowohl horizontale als auch vertikale Inkonsistenzen vorhanden sein, die ein erneutes Durchlaufen des Planungsprozesses notwendig machen. Dabei sollte als Ergebnis der ersten Konsistenzprüfung eine endgültige Festlegung der gesamtunternehmensbezogenen Strategien angestrebt werden. Dadurch können die an die SGE-Leitungen zurückgegeberien Strategievorschläge unmittelbar im Hinblick auf die nun als Datum geltenden übergeordneten Strategien modifiziert und nach weiterer horizontaler Abstimmung zur erneuten überprüfung eingereicht werden. Dieser Abstimmungs-

1 Da hier der grundlegende Aufbau und Ablauf der Planungssysteme im Vordergrund steht, wird auf die Beschreibung dieser Ebene verzichtet. 
prozeB ist so lange zu wiederholen, bis ein als ausreichend erachtetes Konsistenzniveau erreicht ist ${ }^{1}$.

Wenn auch der Kommunikations-, Arbeits- und Zeitaufwand für diese Vorgehensweise der Strategieplanung in Warenhäusern relativ groß erscheint, führt die überwindung der einseitigen Ausrichtung der "top-do-down"- oder der "bottom-up"-Planung zur Motivation, Ziel-, Strategienund Planidentifikation der nachgelagerten (dezentralen) Planungseinheiten ${ }^{2}$. Darüber hinaus verlangt die Komplexität strategischer Marketingprobleme in Warenhäusern, die u.a. in der Notwendigkeit der differenzierten Betrachtung der primären Erfolgsfaktoren "Ware" und "Filiale" begründet liegt, geradezu eine stärkere Berücksichtigung der SGE-spezifischen Besonderheiten, was durch die Planung im Gegenstromverfahren gewährleistet wird. Hierdurch werden insbesondere auch durch informations- und organisationsbedingte Fehler entstehende (Ziel- und) Strategiekonflikte zwischen den Planungsträgern rechtzeitig gelöst ${ }^{3}$. Damit wird eine weitgehende strategienkonsistenz erreicht, die das Risiko einer Suboptimierung in der strategienplanung deutlich verringert ${ }^{4}$.

1 Aufgrund der Vielfalt der Strategieninhalte sowie der beteiligten Personen erscheint die vollständige Realisierung, d.h. eine optimale vertikale und horizontale Abstimmung, als unmöglich. Insofern muß dieser Proze $\beta$ dann abgebrochen werden, wenn ein ausreichendes Konsistenzniveau erreicht ist, d.h. Strategien entwickelt sind, die die zielerreichung des gesamten Warenhauskonzerns sicherstellen.

2 Berücksichtigt man, $d a \beta$ auch bei den anderen Planungsverfahren - bei gleicher Qualität der Planungsergebnisse - vertikale und horizontale Abstimmungsprozesse durchlaufen werden müssen, so wird die negative Beurteilung deutlich relativiert. Vgl. hierzu auch Wild,J., Grundlagen, a.a.O., S. 200; Töpfer, A., a.a.O., S. 116. 3 Vgl. Töpfer, A., a.a.O., S. 116.

4 Vgl. Wild, J., Grundlagen, a.a.0., s. 200. 
Insgesamt fördert die Planung im Gegenstromverfahren insbesondere die vertikale Integration der strategischen Entscheidungen auf den unterschiedlichen Planungsebenen, während sie für die besonders konfliktträchtige horizontale Koordination zwischen Warengruppen- und Filialgruppenleitung in der Sortimentspolitik keine konkreten Abstimmungsmechanismen aufzeigt. Diesem Problembereich sind die nachfolgenden Ausführungen gewidmet.

\subsection{Horizontale Abstimmung (Koordination) von Marke- tingstrategien auf der SGE-Ebene}

Die besondere Brisanz der horizontalen Abstimmung zwischen den Warengruppenleitungen und den Filialgruppenleitungen liegt in ihrer unterschiedlichen Sichtweise begründet. Bei der Warengruppenleitung steht grundsätzlich die "Ware" aus gesamtunternehmensbezogener Sicht im Mittelpunkt. Dagegen basieren die planerischen Bemühungen der Filialgruppenleitungen auf standortspezifischen Besonderheiten, was zur Entwicklung von differenzierten Marketingstrategien führt.

Bei hierarchischer Gleichrangigkeit der Warengruppenund Filialgruppenleitung stellt sich mit der Frage der Koordination der Marketingstrategien zugleich die Frage nach der Verteilung der Planungskompetenzen auf die zentralen (= Warengruppenleitungen) und dezentralen (=Filialgruppenleitungen) Einheiten. Für den Bereich der Sortimentspolitik bedeutet dies in erster Linie die Frage, wer über die Sortimentsstruktur $z u$ entscheiden hat.

In diesem Zusammenhang entwickelt die Warengruppenleitung primär aus zentralseitiger Sicht (wobei z.B. Aspekte der Einkaufsmöglichkeit, des Kaufverbunds usw. dominieren) einen Vorschlag zur Sortimentsstruktur für den 
Warenhauskonzern als Ganzes. Die Filialgruppenleiter entwickeln ihrerseits unter primär filialbezogener Sicht (wobei insbesondere standortspezifische Besonderheiten der Zielgruppen- und Konkurrenzsituation dominieren) eine standortspezifische Sortimentsstruktur. Diese differenzierten Filialgruppensortimente werden in der Regel mehr oder weniger stark von der warengruppenorientierten, durchschnittlichen Sortimentsstruktur abweichen und bedürfen somit einer Abstimmung, die je nach Intensität der Abweichung unterschiedlich umfangreich sein wird.

Dabei treten die wesentlichen strategischen Koordinationsprobleme auf der Artikelgruppenebene auf. Denn die Art und Anzahl der Warengruppen wird bereits bei der übergeordneten strategischen Grobplanung auf der Vorstandsebene festgelegt und auch der SGE-Ebene vorgegeben. Insofern muß die Veränderung der Warengruppenstruktur die Ausnahme bleiben. Dementsprechend sollen alle Filialgruppen die gleichen Warengruppen im Sortiment führen, lediglich die Gewichtung bzw. Auffüllung der Warengruppen durch Artikelgruppen ist variierbar. Auf der Artikelebene ist zu klären, inwieweit Vorstellungen von Filialgruppenleitern, die vom durchschnittlichen Sortiment abweichen, berechtigt sind. Dabei ist zu prüfen, ob die filialgruppenspezifische Konkurrenz- oder Zielgruppensituation das Streichen von Artikelgruppen oder die Hinzunahme normalerweise nicht geführter Artikelgruppen rechtfertigt. Dabei sind eine Vielzahl von Entscheidungskriterien $\mathrm{zu}$ berücksichtigen, die insbesondere die zielerreichung (z.B. Umsatz-, Wachstum-, Flächenziele) sowie die Restriktionen (z.B. Ressourcen, Beschaffungsmöglichkeiten, Einkaufsverbund) betreffen.

Für die konkrete Ausgestaltung der sortimentspolitischen Koordination im Rahmen der Planung im Gegenstromverfahren 
sind unterschiedliche Vorgehensweisen mit differierenden $\underline{\text { Partizipationsgraden }}^{1}$ der Filialgruppen zu diskutieren.

Eine eher zentralistische Variante stellt die Differenzierung in Pflicht- und Wahlsortiment dar ${ }^{2}$. Die Warengruppenleitung entwickelt ein Gesamtsortiment, das in exakt umrissene Sortimentsbausteine differenziert ist. Die Filialgruppenleitung kann die Zusammensetzung ihres Sortiments durch Kombination der entwickelten Bausteine mitbestimmen. Allerdings stellt die Vergabe der jeweiligen Sortimentsbausteine nach filialgruppenspezifischen Kriterien im Grunde keine echte Partizipation dar, da die wesentlichen strategischen Parameter weiterhin von der zentralen Warengruppenleitung bestimmt werden.

Im Rahmen einer echten Partizipation sind mehrere Abstufungen denkbar, die von der Vorgabe eines Pflichtsortiments (oder Kernsortiments) mit zusätzlicher Wahlmöglichkeit bis hin $z$ u einer völligen Freigabe der Wahlmöglichkeiten aus den nach wie vor von der zentralen warengruppenleitung festgelegten Bausteinen reicht.

Der Grundgedanke des Konzepts der filialgruppenspezifischen zusatzsortimente besteht darin, den Filialgruppen relativ weitgehende Freiheit bezüglich der Anpassung an die standortspezifischen Besonderheiten zuzugestehen und dennoch als notwendig erachtete zentrale Aspekte in der Sortimentsstrategie sicherzustellen. Hierzu legt die zentrale Warengruppenleitung ein verbindliches Kernsortiment fest. Es beinhaltet exakt spezifiziert jene Artikelgruppen, die Bestandteil jeder Filialgruppe sein müssen.

1 Vgl. zum Problemkreis des Partizipationsgrades dezentraler stellen im Handel insbesondere Steffenhagen, H. , Strategie, Teil I und Teil II, a.a.O., S. $7 \mathrm{ff..}$

2 Vgl. hierzu Herder, H., Zentralisation, Teil II, a.a.0., S. 10; Steffenhagen, H., Strategie, Teil I, a.a.O., S. 8 . 
Jede Filialgruppe entwickelt zusätzlich zu diesem Kernsortiment ein spezifisches zusatzsortiment, das in freier Planungskompetenz festgelegt wird ${ }^{1}$. Dieses Konzept kann darüber hinaus durch die Bereitstellung eines zusätzlichen, zentralseitig entwickelten Wahlsortiments ergänzt werden.

Im Hinblick auf den notwendigen Partizipationsgrad kann darüber hinaus der Filialgruppenleitung ein Mitspracherecht bei der Planung des Kernsortiments und ein Vorschlagsrecht für das Wahlsortiment eingeräumt werden. Diese Mitspracherechte für die Filialgruppenleitung sichern dabei bereits eine weitgehende horizontale $\mathrm{Ab}-$ stimmung.

Durch die relative Entscheidungsfreiheit der SGE-Leitungen wird zum einen die Motivationswirkung bzw. die Identifikation mit den entwickelten Strategien gefördert und kann zum anderen die bei den Filialgruppen vorhandenen differenzierte und fundierte Marktkenntnis stärkeren Eingang in die Strategienplanung finden. Hiermit ist ein adäquates Planungsverfahren gefunden, das den Grundgedanken der hierarchisch-dualistischen strategischen Marketingplanung entspricht.

Die am Beispiel der Sortimentsstrategie aufgezeigte Problematik läßt sich unmittelbar auf die übrigen strategischen Fragestellungen übertragen. Jeder Problembereich der strategischen Marketingplanung ist durch eine variierende Verteilung der strategischen Planungskompetenz zwischen dex Warengruppen- und Filialgruppenleitung

1 Dies hat zur Konsequenz, daß das Zentralsortiment nicht mehr mit der Summe der Filialgruppensortimente übereinstimmt. Dennoch erlaubt auch dieser Vorschlag die Ausnutzung z.B. von zentralen Einkaufsvorteilen, denn die Beschaffung der Ware, d.h. der Vollzug des Einkaufs, auch der des Zusatzsortiments, kann von der zentralen Warengruppenleitung erfolgen. 
gekennzeichnet. Bei konsequenter Weiterentwicklung bzw. Verfeinerung dieser Planungssystematik ist eine warengruppenspezifische Differenzierung möglich, d.h. die Verteilung der Planungskompetenz variiert zusätzlich zum spezifischen strategischen-Problem (z.B. Zielgruppenabgrenzung, Kommunikationspolitik) in Abhängigkeit von der Warengruppe. Dies eröffnet einerseits der Gesamtleitung (Vorstand) den direkten Durchgriff über alle Planungsebenen, wenn z.B. eine spezifische Warengruppe aus gesamtunternehmenspolitischen Gründen eine strategische Sonderstellung einnehmen sol1 $1^{1}$. Der Vorstand kann dann im Rahmen einer strengeren top-to-down-Planung die Vorgaben enger handhaben und die Freiheitsgrade der Warengruppenund der Filialgruppenleitungen weitgehend beschränken. Hierdurch wird auch die Möglichkeit eröffnet, für spezifische Warengruppen die strategischen Planungskompetenzen über das normale Ausmaß hinaus $z u$ vergrößern (z.B. bei besonders stark von der örtlichen Situation abhängigen Warengruppen) ${ }^{2}$.

Mit diesen Ausführungen sind Ansätze ${ }^{3}$ aufgezeigt, die dazu beitragen, die "Kluft" zwischen dem bestehenden, überwiegend zentral orientierten strategischen Planungs-

1 Als Beispiel ist hier die von Karstadt und Kaufhof verfolgte Strategie des Ausbaus des Sport- und Freizeitbereiches zu nennen (vgl. o.V., Kein Gleichlauf, Warenhäuser kämpfen nach wie vor mit Schwierigkeiten, in: Der Handelsvertreter und Handelsmakler, $\mathrm{Nr}$. 14/15, 30.7.1980, S. 800-802, hier S. 800). In diesem Bereich kann die Warenhausleitung die Marketingstrategien bis zu einem relativ konkreten Formalierungsgrad selbst erarbeiten und den Warengruppen- und Filialgruppenleitungen lediglich die perative Vollzugsplanung zugestehen.

2 Hier ist als Beispiel der gesamte Lebensmittelbereich anzuführen, der bereits heute tendenziell stärker als andere Bereiche der Planungskompetenz der Filialen unterliegt.

3 Das Problem der optimalen Verteilung der Planungskompetenz ist hiermit zweifellos nicht gelöst.

Vgl. hierzu auch die Diskussion bei Steffenhagen, H., Strategie, Teil II, a.a.O., S. 7. 
system und einem in der aktuellen Situation besseren bzw. idealen Planungssystem ${ }^{1} \mathrm{zu}$ überwinden. Aufgrund der hier$\mathrm{zu}$ notwendigen Umorientierung sowohl in der grundsätzlichen Denkweise als auch im organisatorischen Aufbau und Ablauf kann die Durchsetzung bzw. Realisierung des Konzeptes der hierarchisch-dualistischen strategischen Marketingplanung nicht ad-hoc erfolgen, sondern muß kontinuierlich und schrittweise durch die Schaffung entsprechender Voraussetzungen, die aus konzeptioneller Gesamtsicht gesteuert sind, angestrebt werden ${ }^{2}$.

\subsection{Stufenweise Realisation eines hierarchisch-dualisti- schen Marketing-Planungssystems in Warenhäusern}

Die Implementierung des Konzepts der hierarchisch-dualistischen strategischen Marketingplanung bedarf einer systematischen Einbeziehung der Probleme des organisatorischen Wandels ${ }^{3}$. Dementsprechend muß sich die langfristig-strategische Entwicklung der Warenhäuser

1 Dabei darf nicht übersehen werden, daß der $z u$ einem bestimmten Zeitpunkt angestrebte Idealzustand - z.B. die konsequente Verwirklichung des Konzepts der hierarchisch-dualistischen strategischen Marketingplanung - auf der Basis spezifischer Annahmen über die Zukunft entwickelt wurde und somit von Zeit zu zeit selbst einer Uberprüfung bedarf.

2 Damit sind im Grunde die wesentlichen Merkmale des "strategischen Management" angesprochen, das somit wie bereits zu Beginn der Arbeit ausgeführt - als Planungsphilosophie für die Warenhäuser geeignet ist. Vgl. hierzu z.B. Trux, W., Kirsch, W., a.a.O., S. 226 ff..

$3 \mathrm{Vgl}$. hierzu die umfassende Diskussion, literarische Fundierung und Forschungsperspektiven bei Kirsch, W., Esser, W.-M., Gabele, E., Reorganisation, München 1978. 
in einem schrittweisen Planungs- und Lernprozeß ${ }^{1}$ vollziehen, der aus konzeptioneller ${ }^{2}$ Gesamtsicht gesteuert wird. Als Voraussetzung der Implementierung sind auf der Basis einer konsequenten Marketingorientierung insbesondere die Informations- und Kontrollgrundlagen, die Erfahrungen bei der Anwendung von Planungsmethoden, die organisatorischen Regelungen zur Koordination und zur Konfliktlösung sowie die Qualifikation des Management zu nennen.

Die Informationsgrundlagen betreffen zum einen die Gewinnung, Verarbeitung und Aufbereitung marketingspezifischer Informationen insbesondere im Hinblick auf die standortspezifische Kunden-, Konkurrenz- und sonstige Umweltsituation? Dieses primär als Dokumentations- und Planungs-

1 Dies entspricht dem Grundgedanken der geplanten Evolution. Dieser Ansatz ist auf die Beobachtungen bei der Einführung von Management-Informationssystemen zurückzuführen. Er fordert die stetige, schrittweise Veränderung bzw. Systemverbesserung durch geplantes Lernen. Vgl. zur Darstellung des Ansatzes den Sammelband von: Rosove, P.E. (Hrsg.), Developing Computer-Based Information Systems, New York usw. 1967. Zur Übertragung des Ansatzes auf die strategische Planung vgl. z. B. Götzen, E., Kirsch, W., a.a.O., S. 190 ff.; Kirsch, W., Esser, W.-M., Gabele, E., Reorganisation, a.a.O., S. 423 ff.; Roventa, P., a.a.0., S. 97 ff.; Trux, W., Kirsch, W., a.a.O., S. $226 \mathrm{ff}$.

2 Konzeptionell bedeutet in diesem $\mathrm{Zusammenhang,} \mathrm{daß} \mathrm{die}$ Ausgestaltung des strategischen Management und das gesamte strategische Planungssystem nicht vollständig, sondern lediglich in Umrissen als Grobkonzeption skizziert ist. Diese wird im Ablauf der schrittweisen Realisierung stets weiter konkretisiert. Vgl. auch Roventa, P., a.a.O., S. 97 .

3 Vgl. hierzu auch die Aspekte zu Marketing-Informationssystemen z.B. von Meffert, H., Computergestütze Marketing-Informationssysteme, Wiesbaden 1975 sowie Lientz, B.P., Chen, M., Long Range Planning for Information Services, in: LRP, Vol. 13, Febr. 1980, S. 55-61. 
grundlage konzipierte Informationssystem ${ }^{1}$ bedarf zum anderen für strategische Fragestellungen des Ausbaus $z u$ einem warenhausspezifischen Frühwarnsystem ${ }^{2}$. Es beinhaltet jene Indikatoren, die bei einer spürbaren Veränderung wesentliche Auswirkungen auf die Marketingstrategien zeigen und infolgedessen zur rechtzeitigen Kurskorrektur frühzeitig erkannt werden müssen. Insbesondere in der aktuellen, turbulenten Umweltsituation ist es wichtig, sich abzeichnende Diskontinuitäten bereits in einem frühen stadium als schwache $\operatorname{Signale}^{3}$ zu erkennen und in die strategische Marketingplanung mit einzubeziehen. Dabei ist den Entwicklungen auf der Käufer-(z.B. Kaufgewohnheiten, Mobilität, Einstellungen, Altersstruktur, Einkommen usw.) und Konkurrenzseite (z.B. neue Strategien, neue Betriebsformen usw.) besondere Aufmerksamkeit zu widmen, da sie die zentralen Grundlagen für die strategische

1 Damit wird gleichzeitig die Voraussetzung zur Realisierung einer "strategischen Kontrolle" geschaffen, die die Einhaltung bzw. Realisierung der Strategien überwacht und steuert. Vgl. $\mathrm{zu}$ den hiermit verbundenen Problemen: Horovitz, J.H., Strategic Control. A New Task for Top Management, in: LRP, Vol. 12, June 1979 , S. 2-7; Köhler, R., Die Kontrolle strategischer Pläne als betriebswirtschaftliches Problem, in: $\mathrm{ZfB} 46$ (1976), S. 301-318; vgl. in diesem Zusammenhang auch die verstärkte Diskussion über das "Marketing-Controlling". Einen Utberblick über den aktuellen Stand der Forschung gibt: Kiener, J., Marketing-Controlling, Darmstadt 1980.

$2 \mathrm{Vgl}$. die Literaturangaben auf s. 6 der Arbeit. Eine sehr gute Darstellung findet sich zusätzlich bei: Kirsch, W., Trux, W. , Strategische Frühaufklärung und Portfolio-Analyse, in: ZfB-Erg.-Heft 2/1979 ("Frühwarnsysteme"), S. 47-69.

3 Diese Grundgedanken gehen auf Ansoff zurück, der das Konzept der "schwachen Signale" in den Mittelpunkt strategischer Utberlegungen rückt. Vgl. Ansoff, H.I., Weak Signals, a.a.O., S. $129 \mathrm{ff.;}$ derselbe, Planned Management, a.a.O., S. 2 ff.. Hierzu erscheint es wichtig, daß das Management die relevanten Informationen selbst bestimmt. Vgl. hierzu Hahn, D., Klausmann, W., Indikatoren im Rahmen betrieblicher Frühwarnsysteme, in: Ifo-Schnelldienst 35-36/1979, S. 63-69; Rockart, J.F., -opmanager sollen ihren Datenbedarf selbst bestimmen, in: Harvard Manager, II/1980, S. 45-48. 
Marketingplanung in Warenhäusern darstellen ${ }^{1}$.

Die anschließende Entwicklung von Marketingstrategien setzt die Kenntnis und Anwendungserfahrung geeigneter strategischer Planungsmethoden voraus, die die Fülle vorhandener Informationen verdichten und für strategische Entscheidungen handhabbar machen. Dabei ist auch die Portfolio-Methode noch weiter $z u$ verbessern bzw. zu ergänzen. Dies betrifft z.B. die empirische Absicherung der relevanten Einflußfaktoren in Warenhäusern, die Einbettung der Methode in den gesamten Prozeß der Strategieplanung sowie die Verknüpfung mit zusätzlichen Planungsmethoden. Aufgrund der mit der Portfolio-Methode verbundenen Gesamtsicht der Unternehmung sowie der zugleich differenzierten Betrachtung von strategischen Geschäftseinheiten stellt diese Methode einen ersten Schritt auf dem Wege zu einem verbesserten Planungssystem für Warenhäuser dar ${ }^{2}$.

Denn durch die Bildung strategischer Geschäftseinheiten werden wesentliche Impulse für organisatorische Veränderungen deutlich. Diese beziehen sich zum einen auf die Aufbauorganisation (z.B. die organisatorische Verankerung des Warengruppen- und Filialgruppendenkens) und zum anderen auf die Ablauforganisation und zwar insbesondere im Hinblick auf Regelungen zur notwendigen horizontalen und vertikalen Abstimmung von Strategien (z.B. die Bildung von Teams, Ausschüssen, regelmäBigen Planungssitzungen usw.). Die Organisation muB so gestaltet

1 Dabei muß das Frühwarnsystem so ausgestaltet sein, daB es nicht nur Risiken aufzeigt, sondern zugleich auch Chancen rechtzeitig erkennen läßt. Vgl. zu diesen Gedanken auch: Bürgel, H.D., Frühwarnsystem der SEL $A G$, in: $Z \mathrm{fbF}, 32$ (1980), S. 270-275, hier S. 273.

2 Kirsch bezeichnet die Portfolio-Methode als ersten "robusten" Schritt, was besagt, daß durch die Einführung der Portfolio-Methode ein bedeutsamer Schritt im Hinblick auf die Annäherung an das Leitbild eines am strategischen Management orientierten Planungssystems erfolgt. Vgl. Trux, W., Kirsch, W., a.a.u., S. 228. 
werden, daß die entwickelten Marketingstrategien ohne großen Aufwand an Abstimmungs- und Konfliktlösungsprozessen durch die Organisation umgesetzt werden können.

Die organisatorischen Aspekte, aber auch die Erfahrungen mit neuen Planungsmethoden machen deutlich, daß die Entwicklung zu einem neuen, besseren Planungssystem von einem systematischen Management Development begleitet sein muB ${ }^{1}$. Das Management muß mit den Planungstechniken vertraut gemacht und für strategische Fragestellungen sensibilisiert werden sowie seine Konfliktlösungsfähigkeit verbessern. Dies ist insbesondere unter dem Gesichtspunkt der partizipativen Führung bei der Planung im Gegenstromverfahren wichtig, da umfassendere Kompetenzen und höhere Verantwortung eine höhere Qualifikation des dezentralen Management (Warengruppen- und Filialgruppenleiter) erfordern ${ }^{2}$. Diese Verbesserung der Qualifikation trägt ferner dazu bei, sowohl Ängste und Befürchtungen der von Veränderungen Betroffenen abzubauen als auch die spezifischen Kenntnisse nachgelagerter Planungs- und Entscheidungsinstanzen besser zu nutzen.

Diese zentralen Ansatzpunkte zur Realisierung des Konzepts der hierarchisch-dualistischen strategischen Marketingplanung zeigen die notwendigen Veränderungen im Planungssystem der Warenhäuser auf, um die aktuellen strategischen probleme besser handhaben zu können ${ }^{3}$.

1 Dies betont auch Götzen/Kirsch. Vgl. Götzen, G., Kirsch, W. , a.a.O., S. 182 .

2 Diese Ansicht vertritt auch Steffenhagen. Vgl. Steffenhagen, H., Strategie, Teil II, a.a.O., S. 7.

3 Dabei wird nicht verkannt, daß eine Reihe der angesprochenen Probleme noch einer weiteren theoretischen Durchdringung bedürfen (z.B. Frühwarnsysteme, Konflikthandhabungsprozesse, strategische Kontrollsystems usw.) und normative Aussagen nur begrenzt abzuleiten sind (vgl. auch Hinweise zum aktuellen Stand der Planungstheorie im Kapitel 1.1 der Arbeit). 
Aufgrund ihrer Tragweite sind sie nur langfristig im Sinne einer geplanten Evolution erfolgversprechend $z u$ realisieren. Das entwickelte Konzept der hierarchisch-dualistischen strategischen Marketingplanung beschreibt dabei den anzustrebenden Systemzustand und dient zur Steuerung seiner Entwicklung. Das Konzept entspricht dem Grundansatz modernen strategischen Denkens, die übereinstimmung zwischen den internen Möglichkeiten und den externen relevanten Anforderungen unter Berücksichtigung psychologischer und politischer Aspekte herbeizuführen. Basierend auf einer konsequenten Marketingorientierung wird es mit Hilfe dieser Überlegungen den-Warenhäusern gelingen, schrittweise die Voraussetzungen für eine überwindung der aktuellen Krise und damit der Rückgewinnung einer starken Marktposition zu schaffen. 


\section{Literaturverzeichnis}

Abel1, D.F.

Abel1, D.F.

Abe11, D.F. Hammond, J.S.

Ackoff, R.L.

Aghte, $\mathrm{K}$.

Agthe, K.

Ahlert, D.

Ahlert, D.

Ahlert, D.

Ahlert, D.

Pollmüller, D.

Albach, H.
Defining the Business: The Starting Point of Strategic Planning, Englewood Cliffs, N.J., 1980

Stratgic Windows, in: JoM, July 1978, S. 21-26

Strategic Market Planning, Englewood Cliffs, N.J., 1979

Unternehmensplanung, München und Wien 1972

Strategie und Wachstum der Unternehmung, Baden-Baden 1972

Aktuelle Planungsprobleme eines internationalen Unternehmens, in: $\mathrm{ZfbF} 1976$, S. 352-361

Grundzüge des Marketing, Düsseldorf 1980

Probleme der Abnehmerselektion und der differenzierten Absatzpolitik auf der Grundlage der segmentierenden Markterfassung, in: Der Markt 4/1973, S. $103 \mathrm{ff}$.

Absatzförderung durch Absatzkredite an Abnehmer - Theorie und Praxis der Absatzkreditpolitik, Wiesbaden 1972

Die Rechtsordnung als institutioneller Rahmen des Marketing, in: Handbuch Marketing, Bd. 1, Hrsg. Koinike,J., Gernsbach 1978, S. $117 \mathrm{ff}$.

Strategische Unternehmensplanung bei erhöhter Unsicherheit, in: $\mathrm{Z} f \mathrm{~B} 48$ (1978), S. 7o2-715 
Albach, H. Hahn, D. Mertens, P.

Alberts, $K$.

Althoff, T.

Amara, R.

Anders, H.J.

Angehrn, 0 .

Angehrn, $O$.

Ansoff, H.I.

Ansoff, H.I.

Ansoff, H.I.
Frühwarnsysteme, in: ZfB, Erg. Heft $2 / 1979$

"Sortimentsfight statt Flächenklotzerei", in: ASW $8 / 1979$, S. 6-8

Warenhaus und Kaufhaus, in: Tietz, B. (Hrsg.) Handwörterbuch der Absatzwirtschaft, Enzyklopädie der BWL, Bd. IV, Stuttgart 1974, Sp. $2161 \mathrm{ff}$.

Strategic Planning in a Changing Corporate Environment, in: Long Range Planning, Vol. 12, Febr. 1979, S. $2-16$

Analyse des Einkaufsverhaltens, in: Der Einzelhandel 1978/79.Versuch einer Standardbestimmung, Hrsg.: Gesellschaft für Konsum-, Markt- und Absatzpolitik e.V. (GfK), Nürnberg 1978

System des Marketing, Bern/Stuttgart 1973

Handelsmarken und Herstellermarken im Wettbewerb, Stuttgart 1969

Management Strategie, München 1966

The Changing Manager, in: Ansoff, H.I., Declerck, R.P., Hayes, R.C., From strategic Planning to Strategic Management, London usw. 1976, S. 181-197

Managing, Surprise and Discontinuity Strategic Response to Weak Signals, in: $\mathrm{ZfbF} 1976$, S. 129-152 
Ansoff, H.I.

Ansoff, H.I.

Ansoff, H.I.

Ansoff, H.I. Hayes, R.L.

Ansoff, H.I. Declerck, R.P. Hayes, R.L.

Ansoff, H.I. Leontiades, J.C.

Anthony, R.N.

Arbeitsausschuß für Begriffsdefinitionen der Kommission zur Förderung der handels- und absatzwirtschaftlichen Forschung (Hrsg.)

Arbeitskreis "Langfristige Unternehmensplanung" der Schmalenbach-Gesellschaft
Planned Management of Turbulent Change, Working Paper 78-3, European Institute for Advanced Studies in Management, Brüssel 1978

Strategies for Diversification, in: Taylor, B., Wills, Fr. (Hrsg.) Long Range Planning for Marketing and Diversification, London 1971, S. 172-195

Strategic Management, London usw. 1979

Introduction, in: Ansoff, H.I., Declerck, R.P., Hayes, R.C., From Strategic Planning to Strategic Management, London 1976, S. 1-12

From Strategic Planning to Strategic Management, in: Ansoff, H.I., Declerck, R.P., Hayes, R.L. (Hrsg.), From Strategic Plannung to Strate-. gic Management, London 1976, S. 39-78

"Strategic Portfolio Management", Working Paper 76-16, European Institute for Advanced Studies in Management, Brüssel 1976

Planning and Control Systems, Boston 1965

Katalog E, Begriffsdefinitionen aus der Handels- und Absatzwirtschaft, 2. Ausgabe, München 1975, S. 21

Strategische Planung, in: ZfB 1977, S. $1 \mathrm{ff}$. 
Argenti, J.

Aurich, W.

Schröder, H.K.

Ax, A.

Börsig, C.

BAG (Hrsg.)

Balachandran, V. Gensch, D.H.

Ball, R.

Bamberger, I.

Barth, K.

Barth, $K$.
Die Langfristplanung im Unternehmen, München 1970

System der Wachstumsplanung im Unternehmen, München 1972

Praxis der integrierten Unternehmensplanung. Planungsphilosophie und Planungssystem des Unternehmens Mannesmann, in: $\mathrm{Z}_{\mathrm{fbF}}$ (1979) Heft S. 894-925

Unternehmensplanung im Einzelhandel. Erstellt vom BAG-Arbeitskreis "Unternehmensplanung im Einzelhandel", Köln 1977

Lösung des Marketing-Mix-Problems mit Hilfe der geometrischen Programmierung, in: Köhler, R., Zimmermann, H.-J. (Hrsg.), Entscheidungshilfen im Marketing, Stuttgart 1977, S. 449-469

Physical Distribution. A Suitable Case for Treatment, in: LRP, Vol. 13, Febr. 1980, S. 2-11

Grundprobleme und Forschungsansätze der langfristigen $\mathrm{Zielplanung,} \mathrm{in:}$ zO 2/1977, S. 91-99

Rentable Sortimente im Handel. Zufall oder Ergebnis operationaler Entscheidungshilfen? in: Sonderhefte der Mitteilungen des Instituts für Handelsforschung an der Universität zu Köln, hrsg. v. E. Sundhoff, Nr. 26, Köln 1980

Systematische Unternehmungsführung in den GroB- und Mittelbetrieben des Einzelhandels, Göttingen 1976 
Barth, K.

Barth, $\mathrm{K}$.

Bates, A.D.

Batzer, E. Greipel, E.

Bauer, E.

BBE (Hrsg.)

Bearchell, C.A.

Berg, $H$.

Berger, R.

Berger, s.
Die Warenpräsentation in Einzelhandelsunternehmen, in: Mitteilungen des Instituts für Handelsforschung an der Universität zu Köln, hrsg. v. E. Sundhoff, 27. Jg., 1975, Nr. 7, S. 93-97

Planung, in: Der Filialbetrieb als System - das Cornelius-Stüssgen-Modell hrsg. v. Nieschlag, R. und Eckardstein, D., Köln 1972, S. 65-88

The Troubled Future of Retailing, in: Business Horizons, Aug. 1976, S. 22-28

Vor weiteren Marktanteilsverschiebungen im Einzelhandel, in: Ifo- Schnelldienst, 33. Jg., Heft 25, 4. September 1980, S. 3-8

Markt-Segmentierung, Stuttgart 1977

Untersuchung über die Einstellung der Baden-Württembergischen Bevölkerung zu ausgewählten Betriebsformen, stuttgart 1977

Reatiling. A professional approach, New York usw. 1975

Die Herausforderung des sich wandelnden Marktes von morgen, in: Handelsblatt, Nr. 231, 9./10. Dez. 1978, S. 17

Organisation der Unternehmensführung, in: Der Filialbetrieb als System das Cornelius-Stüssgen-Modell, hrsg.v. Nieschlag, R. und Eckardstein, D., Köln 1972, S. 91-145

LadenverschleiB (Store Erosion). Ein Beitrag zur Theorie des Lebenszyklus von Einzelhandelsgeschäften, Göttingen 1977 
Bermann, B. Evans, J.R.

Berthel, J.

Bidlingmaier, $J$.

Bidlingmaier, $J$.

Bidlingmaier, J.

Bircher, B.

Birkigt, $\mathrm{K}$.

Bitterli, R.E.

Blücher, $K$.

Bloom, P.N.

Kotler, P.

Bock, H.H.
Retail Management: A Strategic Approach, New York 1979

Zielorientierte Unternehmenssteuerung, stuttgart 1973

Marketing Bd. 1 und Bd. 2, Reinbek 1973

Zielkonflikte und Zielkompromisse im unternehmerischen Entscheidungsprozeß, Wiesbaden 1968

Begriff und Formen der Kooperation im Handel, in: Absatzpolitik und Distribution, hrsg. v. J. Bidlingm ier, $\mathrm{H}$. Jacobi, W. Uherek, Wiesbaden 1967 S. 353-395

Langfristige Unternehmensplanung, Bern und Stuttgart 1976

Kritische Anmerkungen zu Dhalla und Yuspeh, in: Harvard Manager, Heft 1/1980, S. $79-82$

Aktuelle Marketingprobleme des Warenhauses, in: Entwicklungstendenzen im Handel, hrsg. v. J.S. Krulis-Randa, Bern/Stuttgart 1971, S. 83-89

Zur Organisation der Kaufhof $A G$, in: ZO $3 / 1979$, s. 132-138

Strategies for High Market-Share Companies, in: HBR, Vol. 53, Nov.-Dec. 1975 , S. 63-72

Automatische Klassifikation. Theoretische und praktische Methoden zur Gruppierung und Strukturierung von Daten (Clusteranalyse), Göttingen 1974 
Böcker, F. Merkle, E.

Böckler, M.

Böhler, H.

Bolen, W.H.

Borschberg, E.

Borschberg, E.,

Bruckhaus, M.

Brumm, G.

Bucklin, C.

Bürgel, H.D.

Buskirk, R.H. Buskirk, B.D.
Die Analyse des Sortimentsverbundes, in: Böcker, F., Dichtl, E. (Hrsg.), Erfolgskontrolle im Marketing, Berlin 1975, S. 179-191

Ein unverwechselbares Profil aufbauen, in: Werben \& Verkaufen, Nr. 17/27. April 1979, S. II-VI

Methoden und Modelle der Marktsegmentierung, Stuttgart 1977

Contemporary Retailing, Englewood Cliffs, N.J. 1978

Diversifikations-Strategien in der Distribution, in: Perspektiven des Marketing im Handel, hrsg. v. Ernst-Werner Blümle und Werner Ulrich, Freiburg (Schweiz) 1974, S. 83-103

Die Diversifikation als Wachstumsform der industriellen Unternehmung, Bern 1969

Organisation der Kaufhof AG, in: $\mathrm{ZO}$, $3 / 1977$, S. 123-132

Die Problematik der Marktsegmentierungspolitik im Rahmen des Marketing, Diss. Berlin 1973

Retail Strategy and the Classification of Consumer Goods, in: JoM, Vol. 27, Jan. 1963, S. 50-55

Frühwarnsystem der SEL AG, in: $\mathrm{ZfbF}$ 32 (1980), S. 270-275

Retailing, New York usw. 1979 
Buttler, G.

Buzzell, R.D. Gale, B.T.

Sultan, R.G.

Croon, P.

Chandler, A.D.

Clifford, D.K. Jr.

Cohem, K.J. Cyert, R.M.

Conrads, B.

Cox, W.E. Jr.

Crawford, C.M.
Bevölkerungsrückgang in der Bundesrepublik, Ausmaß und Konsequenzen, Köln 1979

Market Share - a key to profitability in: HBR Jan.-Febr. 1975, S. 97-106

Aids in Determing Strategy, the Internal Anaiysis, in: Long Range Planning, Vol. 12, August 1979, S. 65-73

Strategy and Structure, Cambridge, Mass. 1962

Managing the Product Life Cycle, in: Kotler, P., Cox, K., Marketing Management and Strategy. A Reader, Englewood Cliffs, N.J. 1980, S. 175-181

Management Strategy; Formulation, Implementation and Monitoring, EIASMWorking Paper 72-23

Großbetriebsformen des Einzelhandels, in: Handlexikon für Handel und $\mathrm{Ab}$ satz, hrsg. v. Falk, B., Wolf, I., München 1979

Product Portfolio Strategy: A Review of the Boston Consulting Group Approach to Marketing Strategy, in: AMA 1976, S. 465-47o

Das Leitlinienkonzept in der Absatzplanung, in: Marketingtheorie. Verhaltensorientierte Erklärungen von Marktreaktionen, hrsg. v. Kroeber-Riel, W., Köln 1972 , S. $254-269$

Marktsegmentierung, Eine Analyse zur Zielgruppendefinition unter besonderer Berücksichtigung soziologischer und psychologischer Kriterien, Frankfurt/ Bern 1977 


\author{
Dahlhoff, H.-D. \\ Davidson, W.R. \\ Doody, A.F. \\ Sweeny, D.J. \\ Davidson, W. \\ Day, G.S.
}

Deuss, W.

Dewbold, Fr. D.

Dhalla, N.K. Yuspeh, S.

Dicht1, E.

Diller, $\mathrm{H}$.

Dunst, $K$.

Dunst, $K$.
Kaufentscheidungsprozesse von Familien, Frankfurt 1980

Retailing Management, 4. Aufl., New York 1975

Der Lebenszyklus im Einzelhandel, in: Harvard Manager 2/1979, S. 46-54

Diagnosing the Product Portfolio, in: JoM, Vol. 41, April 1977, S. 29-38

Die Deutschen sind keine Konsummuffel, in: Der Spiegel, 13. Juni 1978, S. $36-41$

Product Portfolio Analysis for U.S. Universities, in: Acron Business and Economic Review, Vol. 11, Nr. 1, Spring 1980, S. 39-45

Abschied vom Konzept des Produktlebenszyklus (dt. Ubersetzung), in: Harvard Manager, Heft $1 / 1980$, S. 69-79

Die Bildung von Konsumententypologien als Grundlage der Marktsegmentierung, in: WiSt, 3. Jg. (1974), Heft 2, S. 54-59

Image-Analyse ohne Spekulation, in: Rationeller Handel, 2 /1978, S. 6-14

Strategisches Portfolio-Management, Berlin/New York 1979

Konkurrenzanalyse, in: Marketing Enzyklopädie, Bd. 2, München 1974, S. 147-154 
Drucker, P.F.

Drumm, H.J.

Ebskamp, K.

Eggert, $\mathrm{K}$.

Eggert, U.

Eggert, U.

Eisenbarth, A.

Enis, B.M.

La Garce, R.

Prell, A.E.

Everitt, B.

Ewing, D.W. (Hrsg.)

Falk, B.R. Wolf, $J$.

Fehr, G.
The Age of Discontinuity, New York 1969

Planungs- und Anpassungsprobleme der Geschäftsbereichsorganisation, in: $\mathrm{Z} f \mathrm{~B} 48(1978)$, S. 87-104

Strategische Planung in der Praxis, in $\mathrm{Zfbf-Kontaktstudium} \mathrm{31(1979),}$

S. 11-20

Fachhandels-Strategie für die 8oer Jahre: Die Marketing-Lücke schließen, in: Rationeller Handel 3/1979, S. 18-23

Profilierungschancen für Fachgeschäfte, in: Rationeller Handel $6 / 1979$, S. $40-44$

Handel '80: Die Zeit der Dinosaurier, in: ASW, $12 / 1978$, S. 90-96

Bedeutung und Problematik des Sonderangebots im Einzelhandel, Diss. München 1968

Extending the Product Life Cycle, in: Business Horizons, 20 (June 1977), S. 46-56

Cluster-Analysis, London 1974

Long-Range Planning for Management, New York 1964

Handelsbetriebslehre, 4. Auf 1., München 1979

Warenhauskonzerne nach einem Jahrzehnt der Umstrukturierung, in: Rationeller Handel, 3/1980, S. 30-32 
Fogg, D.C.

Freese, G.

Freese, G.

Frese, E.

Frese, E.

Frese, E.

Freter, H.

Freudenmann, H.

Gabele, E.

Gälweiler, A.

Gälweiler, A.
Planning Gains in Market Share, in: JoM 38 (July 1974), S. 30-38

Ein Hauch von Harrod's, in: Die Zeit, Nr. 20, 11.5.1979, S. 19

Die Verkäufer kommen zurück. Wie die großen Warenhäuser aus der Flaute herauskommen wollen, in: Die Zeit, $\mathrm{Nr} .23$, 27.5 .1977 , S. 18

Ziele als Führungsinstrumente - Kritische Anmerkungen zum "Management by Objectives", in: zO 5/1971,

S. 227-230

Kontrolle und Unternehmensführung Entscheidungs- und organisationstheoretische Grundlagen, Wiesbaden 1968

Heuristische Entscheidungsstrategien der Unternehmensführung, in: ZfbF 1971, S. $283-306$

Markenpositionierung. Ein Beitrag zur Fundierung markenpolitischer Entscheidungen auf der Grundlage psychologischer und ökonomischer Modelle. Habilitationsschrift Münster 1978

Planung neuer Produkte, Stuttgart 1965

Unternehmensstrategie und Organisationsstruktur, in: ZO 4/1979, S. 183-19o

Marketingplanung im System einer integrierten Unternehmensplanung, in: Poth, L. (Hrsg.) Marketing Bd. 2, Neuwied 1979

Strategische Geschäftseinheiten (SGE) und Aufbauorganisation der Unternehmung, in: ZO 5/1979, S. 252-260 
Gälweiler, A.

Gälweiler, A.

Gerstung, F.

Glinz, M.

Götzen, G. Kirsch, W.

Goodrich, J.N. Hofmann, A.J.

Grochla, E. Szyperski, N. (Hrsg.)

Gröne, A.

Gross , H.

Groh, G.

Gümbel, R.
Unternehmenssicherung und strategische Planung, in: ZfbF 28(1976), S. 362-279

Unternehmensplanung, Frankfurt/New York 1974

Die Servicefunktion als Instrument des Handelsmarketing, Göttingen 1979

Sonderpreisaktionen des Herstellers und des Handels, Wiesbaden 1978

Problemfelder und Entwicklungstendenzen der Planungspraxis, in: ZfB 1979, S. 162-194

"Warehouse Retailing: The Trend of the Future?", in: Business Horizons, April 1979 , S. $45-50$

Model1- und Computergestützte Unternehmensplanung, Wiesbaden 1973

Marktsegmentierung bei Investitionsgütern, Wiesbaden 1977

Markenpolitik, in: Nieschlag, R., Eckardstein, D., Der Filialbetrieb als System - das Cornelius-StüssgenModel1, Köln 1972, S. 211-230

Marktsegmentierung, in: Handwörterbuch der Absatzwirtschaft, Hrsg.: Tietz, B., Stuttgart 1974, Sp. 1408-1420

Die Sortimentspolitik in den Betrieben des Wareneinzelhandels, Köln-Opladen 1963 
Hadaschik, M.

Hahn, D.

Klausmann, W.

Hal1, W.K.

Hamermesh, R.G. Silk, St.B.

Hamermesh, R.G. Anderson, M.J. Harris, J.E.

Hanan, M.

Hanhart, E.W.

Hansen, H.R. (Hrsg.)

Hansen, U.

Hartmann, $\mathrm{K}$.
Die Einsatzbedingungen organisierter langfristiger Unternehmensplanung, Diss. Berlin 1979

Indikatoren im Rahmen betrieblicher Frühwarnsysteme, in: Ifo-Schnelldienst 35-36/1979, S. 63-69

SBU's: Hot, New Topic in the Management of Diversification, in: Business Horizons, Febr. 1978, S. 17-25

How to compete in stagnant industries, in: HBR, Sept.-Oct. 1979, S. 161-168

Unternehmensstrategien bei niedrigen Marktanteilen, in: Harvard Manager $3 / 1979$, S. $40-48$

Reorganize Your Company Around its Markets, in: Kotler, Ph., Cox, K. (Ed.), Marketing Management and Strategy. A Reader; Englewood Cliffs, N.J., 1980, S. $135-145$

Marktgerechte Koordination von Einkauf und Verkauf im Warenhaus, Bern 1967

Computergestützte Marketingplanung, München 1974

Absatz- und Beschaffungsmarketing des Einzelhandels, Band 1 und 2, Göttingen 1976

Die Unternehmensplanung - eine Kette von Fehlprognosen?, in: $\mathrm{ZO}$ $1 / 1979$, S. $2-4$ 


\begin{abstract}
Hartigan, I.A. Clustering Algorithmus, New York 1975
Hauschildt, J .

Hauschildt, I.

Hebbering, B.

Hedley, B.

Hedley, B.

Heemeyer, $\mathbf{M}$.

Zielbildung - ein heuristischer Prozeß, in: $\mathrm{ZfB} 76, \mathrm{~S} .328-340$

Zielhierarchien in innovativen Entscheidungsprozessen, in: Ulrich, $\mathrm{H}$. (Hrsg.) Unternehmensplanung, Wiesbaden 1975 , S. 103-132

Profil im Verkauf. Ein Warenhaus arbeitet an seiner Identität, in: Moderner Markt 3/1979, S. 23-27

Strategy and the "Business Portfolio", in: Long-Range Planning Vol. 10, Febr. 1977 , S. 9-15

A Fundamental Approach to Strategy Development, in: LRP Vol. 9, Dec, 1976 , S. 2-11

Psychographische Marktanalyse im Einzelhandel. Verhaltenswissenschaftliche und meBtheoretische Verankerung einer operationalen Befragungsund Auswertungskonzeption, Diss. Siegen 1980
\end{abstract}

Heinemann, $M$.

Heinen, E.

Heiner, H.,

Henderson, B.R.

Henseler, $\mathrm{R}$.
Die Erfahrungskurve in der Unternehmensstrategie, Frankfurt/New York 1974

Einkaufsstättenwahl und Firmentreue des Konsumenten, Diss. Münster 1974

Das Zielsystem der Unternehmung, 2 . Aufl., Wiesbaden 1971

Bis zu 60 Dienstleistungsangebote für den Warenhauskonzern, in: BdW, Nr. 77, 31.3.1979, S. 5

Imagepolitik in Betrieben des mittelständischen Facheinzelhandels, Göttingen 1978 
Henzler, M.

Henzler, $\mathrm{H}$.

Henzler, $\mathrm{H}$.

Herder, H. von

Herder, H. von

Herder, H. von

Herder, H. von

Herder, H. von

Hill, W.

Hill, W.

Fehlbaum, R.

Ulrich, P.

Hinterhuber, H.H.
Strategisches Marketing als Impulsgeber der 8oer Jahre, in: $\mathrm{ZfbF}$, Sonderheft $11 / 1980$, S. $70-86$

Neue Strategie ersetzt den Zufall, in Manager Magazin, Heft 4/1979, S. 122-129

Strategische Geschäftseinheiten (SGE) Das Umsetzen von Strategischer Planung in Organisation, in: $\mathrm{ZfB} 48$ (1978), S. 912-919

Bedienung - der wiederentdeckte Bedarf?, in: Rationeller Handel 1/1980, S. $13-17$

Filialunternehmen: Alle Macht der Zentrale?, in: Rationeller Handel, 4/1979, S. $2-8$

Filialorganisation: Optimierung mit Kompromissen, in: Rationeller Handel, 5/1979 S. $8-13$

Wie zentralistisch ist mein Betrieb?, in: Rationeller Handel 6/1979, S. 33-39

Determinanten der (de-) Zentralisation, in: Rationeller Handel $1 / 1980$, S. 2-6

Umweltanalyse und Unternehmensplanung, in: Die Unternehmung 77, S. 289-305

Organisationslehre, 2. Aufl., Bern und Stuttgart 1976

Strategische Unternehmensführung, 2 . Aufl., Berlin, New York 1980 


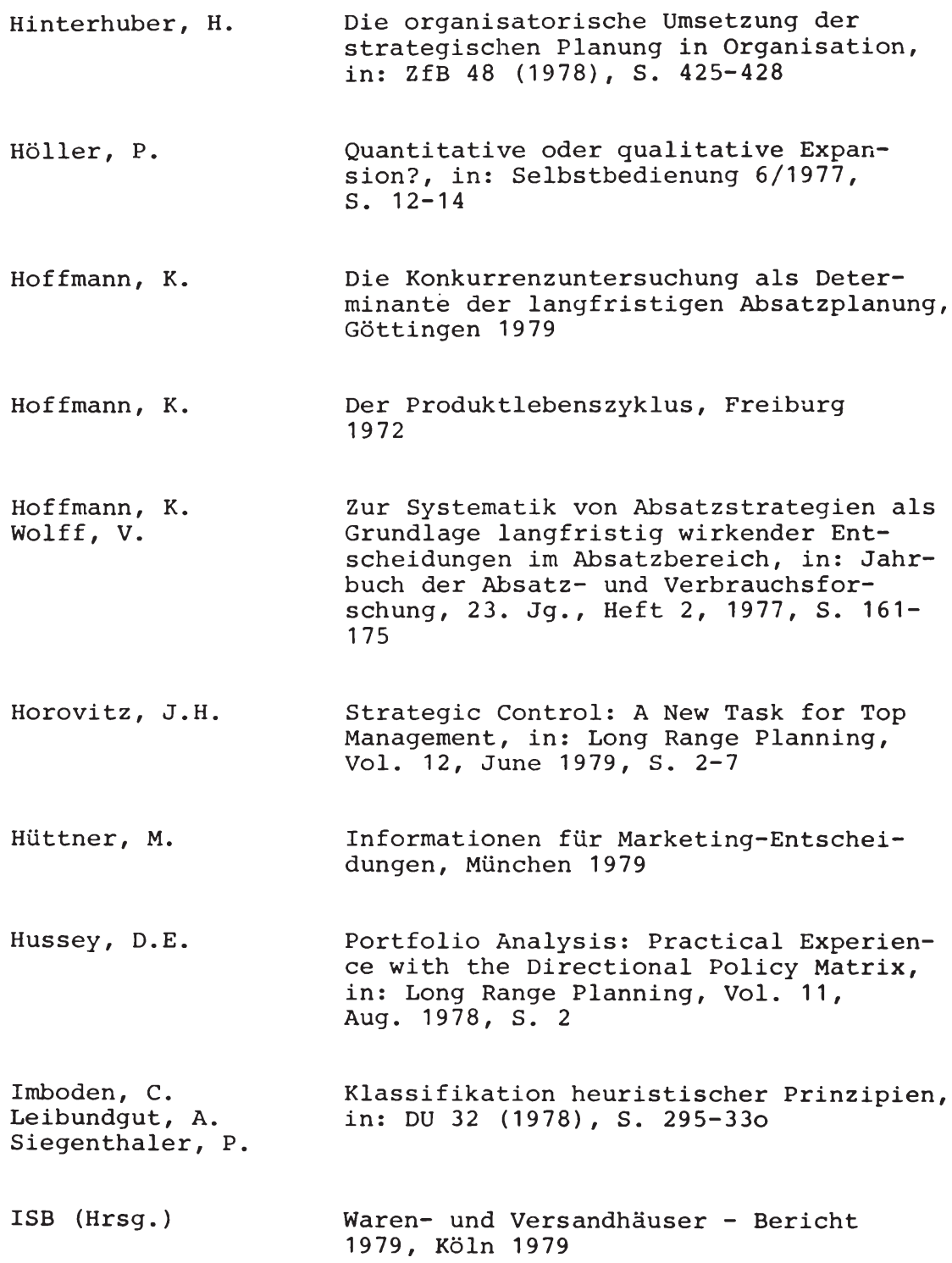
ce with the Directional Policy Matrix, in: Long Range Planning, Vol. 11, Aug. 1978, S. 2

Imboden, $\mathrm{C}$.

Leibundgut, A.

Klassifikation heuristischer Prinzipien, Siegenthaler, P. in: DU 32 (1978), S. 295-330

ISB (Hrsg.)

Waren- und Versandhäuser - Bericht 1979, Köln 1979 


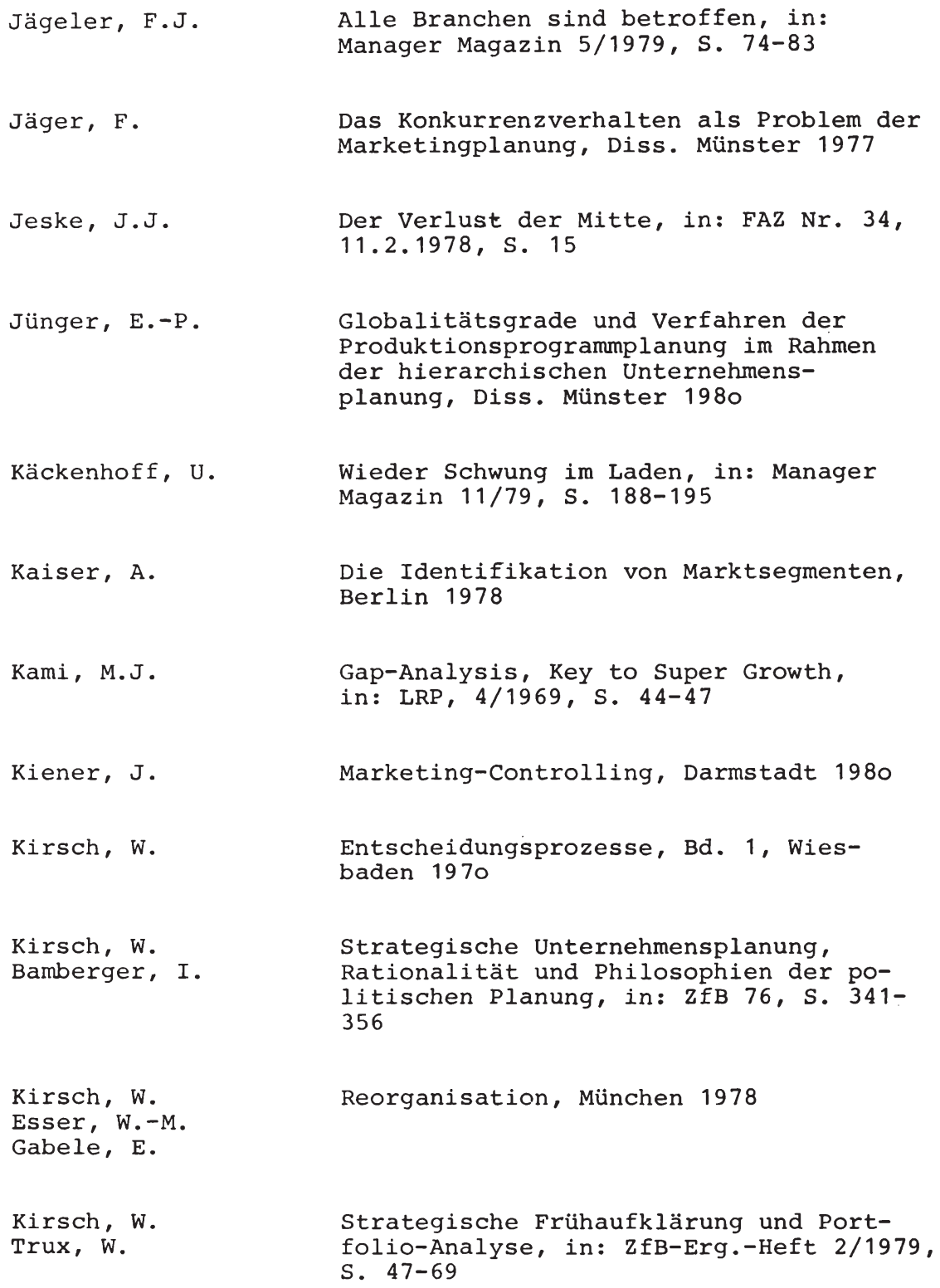

Kirsch, W. Esser, W. - M. Gabele, E.

Kirsch, W. Trux, W.

Reorganisation, München 1978

Strategische Frühaufklärung und Portfolio-Analyse, in: ZfB-Erg.-Heft 2/1979, S. 47-69 
Klein, H.K.

Klein-Blenkers, F.

Klein-Blenkers, F.

Koch, H.

Koch, H.

Koch, $\mathrm{H}$.

Koch, H.

Köhler, R.

Köhler, R.
Heuristische Entscheidungsmodelle, Wiesbaden 1971

Imagepolitik im mittelständischen Facheinzelhandel, in: Handelsforschung heute. Festschrift zum 5ojährigen Bestehen der Forschungsstelle für den Handel, Schriftenreihe der Forschungsstelle für den Handel, Dritte Folge, Nr. 7, Berlin 1979, S. 125-136

Unternehmerziele im Facheinzelhandel, in: Mitteilungen des IfH an der Universität zu Köln, hrsg. v. E. Sundhoff, 24. Jg., 1972, Heft 7/8, S. 69-75 und $81-83$

Zum Verfahren der strategischen Programmplanung, in: $2 \mathrm{fbF}, 31$ (1979). S. $145-161$

Aufbau der Unternehmensplanung, Wiesbaden 1977

Wirtschaftsunruhe und Unternehmensplanung, in: $\mathrm{zfbF} 1976$, S. 330-341

Die zentrale Globalplanung als Kernstück der integrierten Unternehmensplanung, in: Wild, J. (Hrsg.), Unternehmensplanung. Reader + Abstracts, Reinbek 1975, S. 145-153

Marketing-Entscheidungsn als Anwendungsgebiet der quantitativen Planung, in: Köhler, R., Zimmermann, M.-J., Entscheidungshilfen im Marketing, Stuttgart 1977, S. 2-28

Die Kontrolle strategischer Pläne als betriebswirtschaftspolitisches Problem, in: $\mathrm{z} f \mathrm{~B} 76, \mathrm{~s} .301-318$ 
Köhler, R. Uebele, $H$.

Kook, W.

Kotler, P.

Krüger, $\mathrm{H}$.

Krautter, I.

Kreikebaum, H.

Kreikebaum, $\mathrm{H}$.

Kühn, R.

Kuhlmann, E.

Kupsch, P.

Kunt, w.
Planungstechniken: Daumen oder EDV, in: ASW $1 / 79$, S. 62-71

Einstellungen zur Universität - Operationalisierung und empirische prüfung verhaltenswissenschaftlicher Hypothesen am Beispiel der Stadtbevölkerung von Münster, Frankfurt 1980

Marketing Management. Analysis Planning and Control, 4. Aufl., Englewood Cliffs, N.J., 1980

Warenhäuser mit neuer "Philosophie", in: Markenartikel 2/79, S. 62-67

Zum Problem der optimalen Marktsegmentierung, in: ZfB 1975, S. 109-128

Die Lückenanalyse als Voraussetzung der Unternehmensplanung, in: Interne Revision 1/1973, S. 17-26

Die Lückenanalyse als Voraussetzung der Unternehmensplanung, in: Interne Revision $1 / 1973$, S. 17-26

Marketing-Mix, in: Poth, L.G. (Hrsg.), Marketing 3, Neuwied 1979

Die Selektion von Segmentierungsmerkmalen, Arbeitspapier zum Marketing, Bd. 4, Hrsg.: Engelhardt, H.W., Hammann, P., Bochum 1979

Unternehmensziele, Stuttgart/New York 1979

Die Zielkonzeption der mittelständischen Einzelhandelsunternehmer, Sonderheft der Mittellungen des Instituts für Handelsforschung an der Universität Köln, hrsg. v. E. Sundhoff, Göttingen 1975 
Lessing, $R$. Welke, W.

Lambin, S.-J.

Langmantel, E.

Lankamm, T.

Levitt, T.

Lientz, B.P. Chen, $M$.

Lo, L.

Lorange, P.

Lorange, P. Vancil, R.F.

Mace, D.

Mangold, H.G.
Marketing, Bd. 2 der Schriftenreihe Strategische Unternehmensführung, Hrsg. G. Kienbaum, München 1976

Ein On-Line-Modell zur computergestützten Planung des Marketing-Mix, in: Köhler, R., Zimmermann, H.-J. (Hrsg.), Entscheidungshilfen im Marketing, stuttgart 1977, S. 430-447

Strukturwandel in der Bundesrepublik, in: Ifo-Schnelldienst $34 / 1979, \mathrm{~S} .12-20$

Zielkonflikte in der Unternehmensplanung, in: Harvard Manager 3/1980, S. $92-97$

Exploit the Product Life Cycle, in: HBR, Vol. 43, Nov.-Dec. 1965, S. 81-94

Long Range Planning for Information Services, in: LRP, Vol. 31 , Febr. 1980 , S. 55-61

Prognoseinformationen für kundendienstpolitische Entscheidungen, dargestellt an Beispielen des Fotomarktes, Diss. Münster 1979

Corporate Planning. An Executive Viewpoint, Englewood Cliffs, N.J., 1980

How to design a Strategic Planning System, in: HBR, Sept.-Okt. 1976, S. $75-81$

Long Range Planning in Retailing, in: Wills, G., New Ideas in Retail Management London 1970, S. 21-38

Die Absatzpolitik der deutschen Warenhaus-Gesellschaften, Berlin 1971 
Mathieu, G.

Meffert, $H$.

Meffert, $H$.

Meffert, $H$.

Meffert, $H$.

Meffert, $H$.

Meffert, $\mathrm{H}$.

Meffert, $H$.

Meffert, $\mathrm{H}$.

Meffert, $H$.
Lebenszyklen als Entscheidungshilfe für Betriebstypen im Handel, in: Marketing-Journal 2/1978, S. 122-128

Marktsegmentierung und Standardisierung als absatzpolitische Probleme internationaler Unternehmungen, in: Zeitschrift für Wirtschafts- und Sozialwissenschaften, Heft $4 / 1980$ (in Vorbereitung)

Marketing. Einführung in die Absatzpolitik, 5. Aufl., Wiesbaden 1980

Perspektiven des Marketing in den 8 oer Jahren, in: DBW 4o (1980), Heft 1, S. $59-80$

Marktführer in gesättigten Märkten, in: ASW 7/1980, S. 54-59

Strategische Planung in gesättigten, rezessiven Märkten, in: ASW 6/1980, S. $89-97$

Der Markenartikel und seine Bedeutung für den Verbraucher, in: Gruner + Jahr, Schriftenreihe Band 24, Hamburg 1979

Bedeutung, Aufgaben und Zukunftsperspektiven des Ladenbaus im Rahmen des Handelsmarketing, Münster 1979 (unveröffentlichtes Manuskript des Instituts für Marketing)

Marktsegmentierung und Marktwahl im internationalen Marketing, in: DBW, 37. Jg. (1977), S. 433-446

Die Durchsetzung von Innovationen in der Unternehmung und im Markt, in: ZfB 46 (1976), S. 77-100 
Meffert, $H$.

Meffert, $H$.

Meffert, $\mathrm{H}$.

Meffert, $H$.

Meffert, $H$. Dahlhoff, H. D.

Meffert, $H$. Steffenhagen, $\mathrm{H}$.

Meffert, $H$. Steffenhagen, $\mathrm{H}$.

Melcher, P.

Mockler, R.I.

Moser, D.
Computergestützte Marketing-Informationssysteme, Wiesbaden 1975

Zum Problem des Marketing-Mix.

Eine heuristische Vorauswahl absatzpolitischer Instrumente, in: Marketing heute und morgen, Hrsg. H. Meffert, Wiesbaden 1975 , S. 257-275

Interpretation und Aussagewert des Produktlebenszykluskonzepts, in: Neuere Ansätze der Marketingtheorie, Festschrift zum 80. Geburtstag von Otto Schnutenhans, Hrsg.: Hammann, P., Kroeber-Riel, W., Meyer, C.W., Berlin 1974 , S. $84-134$

Unternehmensziele, in: Jahrbuch des Marketing, Ausgabe 1971, Hrsg.:

Schöttle, K.M., Essen 1971, S. 22-34

Kollektive Kaufentscheidungsprozesse von Konsumenten, in: Handelsforschung heute, Festschrift zum 5ojährigen Bestehen der Forschungsstelle für den Handel, Berlin 1979, S. 193-205

Marketing-Prognosemodelle, Stuttgart 1977

Konflikte zwischen Industrie und Handel, Wiesbaden 1976

Kundendienstleistungen als Instrument der Absatzpolitik im Kauf- und Warenhaus, Stuttgart 1972

Theory and Practice of Planning, in: HBR, March-April 1970, S. 149-159

Neue Betriebsformen im Einzelhandel, Frankfurt/Zürich 1974 
Müller-Hagedorn

Nagtegaal, $\mathrm{H}$.

Nauer, E.

Neubauer, F. F.

Neubauer, F. F.

Nieschlag, R.

Dichtl, E.

Hörschgen, H.

Oehme, W.

o.v.

o.V.

o.V.

o.V.
Bevorzugte Betriebsformen des Einzelhandels und das Lebenszykluskonzept, in: $\mathrm{zfbF}$ 3o (1978), S. 106-124

Wie überlebt mein Unternehmen?. Wiesbaden 1975

Standortmodell und Standortpolitik im Einzelhandel, Bern/Stuttgart 1970

Strategien besser absichern, in: Manager-Magazin 12/79, S. 154-159

Wer wird durch die Rezession besonders hart betroffen?, in: Blick durch die Wirtschaft, Nr. 90, 17. April 1980, S. 3

Marketing, 10. Aufl., Berlin 1979

Unternehmensführung im Handelsbetrieb, Wiesbaden $0 . \mathrm{J}$.

Sear's New 5-Year-Plan: To Serve Middle America, in: Kotler, P., Cox, K., Marketing Management and Strategy. A Reader, Englewood Cliffs, N.J., 1980, S. $248-256$

Kein Gleichlauf, Warenhäuser kämpfen nach wie vor mit Schwierigkeiten, in: Der Handelsvertreter und Handelsmakler, Nr. 14/15, 30.7.1980, S. 800-802

Der "neue Horten" will Freizeitspezialist sein, in: FAZ, $\mathrm{Nr} .124,30$. Mai 1980, S. 17

Der Kaufhof will Vorwärtsstrategie beibehalten, in: FAz, Nr. 116, 20. Mai 1980, S. 13 
o.v.

o.V.

o.V.

Payne, B.

Patel, P. Younger, $M$.

Penrose, E.T.

Petermann, G.

Plötzeneder, H.D. (Hrsg.)

Prognos AG
Pümpin, C.B.

Raithel, $\mathrm{H}$.

Rieser, I.
Nichts geht durch die Lappen, in:

Manager Magazin 9/1979, S. 46-57

Wachstum durch Lebensqualität, in: Wirtschaftswoche Nr. 39, 22.9.1978, S. 16-24

Warenhäuser 77: Zu Euphorie besteht kein AnlaB, in: Rationeller Handel, Aug. 1977, S. 41-45

Planning for Company Growth of the Firm, New York 1963
A Frame of References for Strategy Development, in: LRP, April 1978, S. $6-12$

The Theory of the Growth of the Firm, Oxford 1966

\begin{abstract}
Marketinginstrumentarium im Handel, in: Handelsforschung heute, Festschrift zum 5ojährigen Bestehen der Forschungsstelle für den Handel, Berlin 1979, S. 153-166
\end{abstract}

Computergestützte Unternehmensplanung, Stuttgart usw. 1977

Prognos-Report Nr. 19, Zürich 1979

Langfristige Marketingplanung, Bern/ Stuttgart 1968

Innovation ohne Namen, in: Manager Magazin 2/79, S. 52-59

Frühwarnsysteme für die Unternehmungspraxis, München 1980 
Robinson, S.J. Hichens, R.E. Wade, D.P.

Rockart, J.F.

Rohlmann, P.

Roventa, P.

Sandler, G.G.

Scheele, W.

Schenk, D.

Schenk, H. -O.

Schiers, I.

Scheuch, F.

Scheuplein, H.
The directional policy matrix-tool for strategic planning, in: Long-Range Planning, Vol. 11, June 1978, S. 8-15

Topmanager sollen ihren Datenbedarf selbst definieren, in: Harvard Manager II/1980, S. 45-58

Marketing in der Rezession, Wiesbaden 1977

Portfolio-Analyse und strategisches Management, München 1979

Die Bedeutung der qualitativen Innovation für die Sortimentsbildung aus der Sicht der Industrie, in: Selbstbedienung $6 / 77$, S. 21-27

Kommunikationspolitik, in: Nieschlag, R. Eckardstein, D., Der Filialbetrieb als System. Das Cornelius-Stüssgen-Modell, Köln 1972, S. 261-262

Hersteller- und Handelsmarken im Wandel ihrer gegenseitigen Beziehungen, in: Der Markenartikel, 2/1970, S. 39-47

Polarisierung - die neue Handelsstrategie, in: Markenartikel 10/79, S. 578589

Muß Bonn mehr planen, in: Manager Magazin 5/80, S. 89-101

Heuristische Entscheidungsprozesse in der Produktpolitik, Berlin 1977

Unternehmensstrategien, Wiesbaden 1970 
Schmitt-Grohé, J.

Produktinnovation. Verfahren und organisation der Neuproduktplanung, Wiesbaden 1972

Schmidt-Sudhoff, U. Unternehmensziele und unternehmerisches Zielsystem, Wiesbaden 1967

Schnieder, D.

Zur Problematik der Zielbestimmung in Marketingkooperationen, in: Modernes Marketing - Moderner Handel, hrsg.v. J. Bidlingmaier, Wiesbaden 1972,

S. $89-98$

Schoeffler, S. Buzzell, R.D. Heany, D.F.

Impact of strategic planning on profit performance, in: HBR March-April 1974, S. $134 \mathrm{ff}$.

Schröder, B.

Das Warenhaus der zukunft (II), in: Blick in die Wirtschaft $\mathrm{Nr} .128$, 21.6 .1978 , S. 1

Schuchard-Fischer, C. Multivariate Analysemethoden, Berlin usw. 1980

Scott, B.W. Long-Range Planning in American Industry, New York 1965

Seeringer, $w$.

Ein Ansatz zur Lösung des Marketing-MixProblems, in: JAVF, 1977, S. 143-160

Shanklin, W.L.

Strategic Business Planning: Yesterday, Today, and Tomorrow, in: Business Horizons, Oct. 1979 , S. 7-14

Simmerl, M.

Portfolio-Selektion: Strategie gegen Zufallserfolge, in: ASW 21. Jg. (1978) Heft 5, S. 78-82

slatter, S.St.

Strategic Planning for Public Relations, in: LRP, Vol. 13 June 1980, S. 57-6o

Smit, J. Rade, N.L.

Rational and Non-Rational Planning, in: LRP, Vol. 13, April 1980, S. 87-101 
Spannagel, R.

Spiegel, B.

Springer, C.H.

Stahl, P.

Stalp, H. -G.

Steckenborn, I.

Steffenhagen, $\mathrm{H}$.

Steffenhagen, $\mathrm{H}$.

Steffenhagen, $\mathrm{H}$.

Steiner, G.A.

Steinhausen, D.

Langer, $\mathrm{K}$.

Stoff, W. D.
Strukturwandel im Einzelhandel durch Fußgängerzonen, in: FfH Mitteilungen, Neue Folge, Januar 1979

Die Struktur der Meinungsverteilung im sozialen Feld, Bern und Stuttgart 1961

Strategic Management in General Electrix, in: Operations Research 1973, S. 1177- 1182

Verbundwirkungen im Sortiment, Diss. Münster 1977

Strategische Geschäftseinheiten?, in: ZfB 48 (1978)，S. 919-924

Handelsmarken wachsen im Windschatten, in: Rationeller Handel, 3/1976, S. 48-52

Marktführer im Zwielicht, in: Moderner Markt, 5/1979, S. 22-32

Zukunft erfordert Strategie, in: SB-Warenhaus, 4/1979 (Teil I), S. 6-10 5/1979 (Teil II), S. 6-7

Wirkungen absatzpolitischer Instrumente, Theorie und Messung der Marktreaktion, stuttgart 1978

Top Management Planning, London 1969

Clusteranalyse, Berlin/New York 1977

Marktposition und Unternehmensstrategie, in: Die Unternehmung 1/1978, S. $1-13$ 
Streim, H.

Szyperski, N.

Szyperski, N.

Szyperski, N. Welters, $K$.

Szyperski, N. Winand, $U$.

Teichmann, H.

Thanheiser, H. Patel, P.
Heuristische Lösungsverfahren. Versuch einer Begriffsklärung, in: Zeitschrift für Operations Research, Bd. 19 (1975), S. 143-162

Das Setzen von Zielen - Primäre Aufgabe der Unternehmensleitung, in: $\mathrm{ZfB} 41 /$ 1971 , S. 639-67o

Planurigswissenschaft und Planungspraxis, in: Wild, J. (Hrsg.) Unternehmensplanung, Reinbek 1975, S. 58-75

Grenzen und Zweckmäßigkeit der Planung, in: Die Unternehmung 1976, S. 165-283

Strategisches Portfolio-Management; Konzept und Instrumentarium, in: $\mathrm{zfbF}$ 1978 , S. 123-132

Planungsprobleme der Zentralen im dezentralisierten Unternehmen und Lösungsmöglichkeiten für die Praxis, in: DBW 39 (1979), Heft 3, S. 377-395

Strategische Planung in diversifizierten deutschen Unternehmen, Wiesbaden 1977

The Boston Consulting The Star of the Portfolio Perspective Group (Hrsg.) $\mathrm{Nr} .22$, München 1979

The Boston Consulting Anatomy of the Cash Cow, Perspective Group (Hrsg.) Nr. 25, München 1976

The Boston Consulting Das Portfolio, Perspektiven Nr. 19, Group (Hrsg.) München 1977

Theuer, G. Namenlose Billigsortimente oder Up-Trading?, in: Markenartikel 1/80, s. 20-22 
Thies, G.

Thumme1, D.

Tietz, B.

Tietz, B.

Töpfer, A.

Topritzhofer, E.

Topritzhofer, E.

Trux, W. Kirsch, W.

Ulrich, H.

Ulrich, W.

Unkelbach, W. Wiegmann, V.T.
Vertikales Marketing. Marktstrategische Partnerschaft zwischen Industrie und Handel, Berlin, New York 1976

Entwicklung einer Konzeption zur Bestimmung des langfristig-strategischen Marketing-Mix, Bern-Stuttgart 1972

Wie Verbraucher über Einzelhandelsleistungen denken, in: ASW 3/1978, S. 47-53

Die Standort- und Geschäftsflächenplanung im Einzelhandel, Rüschlikon-Zürich 1969

Planungs- und Kontrollsysteme industrieller Unternehmungen, Berlin 1976

Zur pragmatischen Brauchbarkeit marginal-analytischer Marketing-Mix-Modelle, in: Köhler, R., Zimmermann, H.-J. (Hrsg.), Entscheidungshilfen im Marketing, Stuttgart 1977, s. 430-447

Marketing-Mix, in: Handwörterbuch der Absatzwirtschaft, hrsg. v. Tietz, B., Stuttgart 1974, Sp. 1247-1264

Strategisches Management oder die Möglichkeit einer "wissenschaftlichen Unternehmensführung", in: DBW 1979, S. 215-235

Unternehmenspolitik, Bern/Stuttgart 1978

Einführung in die heuristischen Methoden des Problemlösens, in: WISU $6 / 1976$, S. 63-68

Sortimentssieb für Warenhäuser, in: ASW 7/1978, S. 61-63 
Vancil, R.E. et al (Hrsg.)

Weber, J.A.

Welters, $\mathrm{K}$.

Welzel, K.

Wesner, E.

Wild, J.

Wind, Y.

Winkler, A.

Wissema, J.G.

Van der Pol, H.W. Messner, H.M.

Wittek, B.F.

Wotzka, P.

wündrich, H.

Zahn, E.

Ziegenbein, $\mathrm{K}$.
Formal Planning Systems, Harvard Business Schools, Boston 1969

Planning Corporate Growth with Inverted Product Life Cycles, in: Long Range Planning, Vol. 9, Okt. 1976, S. 12-29

Zum Problembegriff einer Theorie strategischer Unternehmensplanung, in: ZO 3/1978, S. 135-142

Marketing im Einzelhandel, Wiesbaden 1974

Die Planung von Marketing-Strategien auf der Grundlage des Modells des Produktlebenszyklus, Diss. Berlin 1977

Grundlagen der Unternehmensplanung, Reinbek bei Hamburg 1974

Product Portfolio Analysis: A New Approach to the Product Mix Decision, in: AMA 1976, S. 460-464

Kritische Uberlegungen zur Tragfähigkeit des Image-Begriffs, in: JAVF, 19. Jg. (1973), Nr. 2, S. 147-156

Strategic Management archetypes, in: Strategic Management Journal, Vol. 1 $(1980)$, S. $37-47$

Strategische Unternehmensführung bei Diversifikation, Berlin/New York 1980

Standortwahl im Einzelhandel, Hamburg 1970

Strategie-Begriff kritisch betrachtet, in: ASW $11 / 1977$, S. $84 \mathrm{ff}$.

Strategische Planung zur Steuerung der langfristigen Unternehmensentwicklung, Berlin 1979

Der Boston-Effekt, in: ASW, 12/1975, S. 72-75 
Friedrich Wehrle - 978-3-631-75094-0

Downloaded from PubFactory at 01/11/2019 08:26:46AM

via free access 


\section{SCHRIFTEN ZUM MARKETING}

Band 1 Friedrich Wehrle: Strategische Marketingplanung in Warenhäusern. Anwendung der Portfolio-Methode. 1981. 2. Auflage. 1984.

Band 2 Jürgen Althans: Die Übertragbarkeit von Werbekonzeptionen auf internationale Märkte. Analyse und Exploration auf der Grundlage einer Befragung bei europaweit tätigen Werbeagenturen. 1982.

Band 3 Günter Kimmeskamp: Die Rollenbeurteilung von Handelsvertretungen. Eine empirische Untersuchung zur Einschätzung des Dienstleistungsangebotes durch Industrie und Handel. 1982.

Band 4 Manfred Bruhn: Konsumentenzufriedenheit und Beschwerden. Erklärungsansätze und Ergebnisse einer empirischen Untersuchung in ausgewählten Konsumbereichen. 1982.

Band 5 Heribert Meffert (Hrsg.): Kundendienst-Management. Entwicklungsstand und Entscheidungsprobleme der Kundendienstpolitik. 1982.

Band 6 Ralf Becker: Die Beurteilung von Handelsvertretern und Reisenden durch Hersteller und Kunden. Eine empirische Untersuchung zum Vergleich der Funktionen und Leistungen. 1982.

Band 7 Gerd Schnetkamp: Einstellungen und Involvement als Bestimmungsfaktoren des sozialen Verhaltens. Eine empirische Analyse am Beispiel der Organspendebereitschaft in der Bundesrepublik Deutschland. 1982.

Band 8 Stephan Bentz: Kennzahlensysteme zur Erfolgskontrolle des Verkaufs und der Marketing-Logistik. Entwicklung und Anwendung in der Konsumgüterindustrie. 1983.

Band 9 Jan Honsel: Das Kaufverhalten im Antiquitätenmarkt. Eine empirische Analyse der Kaufmotive, ihrer Bestimmungsfaktoren und Verhaltenswirkungen. 1984. 
Friedrich Wehrle - 978-3-631-75094-0

Downloaded from PubFactory at 01/11/2019 08:26:46AM

via free access 